\title{
Don et transplantation d'organes en Suisse
}

Enjeux historiques et sociologiques (1945-2020)

Sous la direction de Raphaël Hammer Vincent Barras

Manuel Pascual 



\section{Don et transplantation d'organes en Suisse}

Enjeux historiques et sociologiques (1945-2020)

Sous la direction de Raphaël Hammer Vincent Barras Manuel Pascual 


\section{CMS / Collection Médecine Société}

CMS est née en 1999, placée sous la responsabilité d'un Comité de rédaction constitué de personnalités scientifiques d'orientations multiples. Les volumes publiés proposent une réflexion pluridisciplinaire, se rapportant à l'ensemble des questions de santé et de médecine envisagées dans leur sens le plus large. L'accent est mis autant sur les implications pratiques que sur les enjeux théoriques, et sur le croisement pluriel des perspectives, qu'elles soient sociologiques, médicales, juridiques, éthiques ou historiques.

CMS se veut le témoin engagé des débats contemporains sur la maladie, la santé et leurs implications sociales, politiques, économiques ou culturelles, et s'adresse à un public comprenant tout à la fois l'ensemble des professionnel-le·s de la santé et de la médecine et tou·te·s celles et ceux qui se sentent concerné·ess par ces enjeux.

Comité de rédaction

Anne-Françoise Allaz, Gaspard Aebischer, Vincent Barras, Patrice Guex, Claude-François Robert, Raphaël Hammer, Bertrand Kiefer

Directeur de collection

Vincent Barras 


\section{Remerciements}

Les résultats présentés dans cet ouvrage sont issus d'une recherche financée par le Fonds national suisse de la recherche scientifique, codirigée par Raphaël Hammer (Haute École de Santé Vaud, Haute École spécialisée de Suisse occidentale - HES-SO), Vincent Barras (Institut des humanités en médecine, CHUV et Faculté de biologie et de médecine, Université de Lausanne) et Manuel Pascual (Centre de transplantation d'organes, CHUV et Faculté de biologie et de médecine, Université de Lausanne), avec la collaboration scientifique d'Alexia Cochand et François Kaech.

Pour leur soutien et leur concours à différentes étapes de la réalisation du projet, nous tenons à remercier vivement les institutions suivantes: le Fonds national suisse de la recherche scientifique, la Haute École de Santé Vaud, la Haute École spécialisée de Suisse occidentale, l'Institut des humanités en médecine, le CHUV et l'Université de Lausanne, Swisstransplant, l'Office fédéral de la santé publique, la Société suisse de néphrologie, les Archives de l'État de Berne, de Bâle et de Saint-Gall, les archives centrales des HUG, les Archives cantonales vaudoises, ainsi que le service de prêt de la Bibliothèque nationale. Enfin, nous sommes très reconnaissants envers toutes les personnes et les collègues qui ont accepté de donner de leur temps lors des entretiens réalisés dans le cadre de cette recherche. 
Cet ouvrage est publié avec le soutien du Fonds national suisse de la recherche scientifique (FNS) et de la Haute école spécialisée de Suisse occidentale (HES-SO)

\section{FNDNF \\ FONDS NATIONAL SUISSE \\ DE LA RECHERCHE SCIENTIFIQUE}

\section{Hes.so}

Haute Ecole Spécialisée

de Suisse occidentale

Fachhochschule Westschweiz

University of Applied Sciences and Arts
Western Switzerland

Couverture et mise en page: Jennifer Freuler

Georg Editeur

Genève

www.georg.ch

Pour leurs textes @ 2021 R. Hammer, V. Barras, M. Pascual

ISBN (papier): 9782825712276

ISBN (PDF): 9782825712283

ISBN (XML): 9782825712290

DOI: 10.32551/GEORG.12276

Cet ouvrage est publié sous la licence Creative Commons CC BY-NC-ND (Attribution - Pas d'Utilisation Commerciale - Pas de Modification)

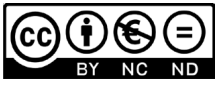




\section{Sommaire}

Remerciements

Liste des auteurs

Introduction.

Le don et la transplantation d'organes dans le regard des sciences sociales et historiques

Raphaël Hammer et Vincent Barras

1. De l'imaginaire à la réalité contemporaine des greffes

2. Enjeux symboliques et résistance culturelle

3. "Don» et discours publics

4. Aspects sociopolitiques et spécificités nationales

5. Le don et la transplantation d'organes en tant que "problèmes publics"

6. Structure de l'ouvrage

Partie l.

L'émergence institutionnelle et publique du don et de la transplantation d'organes

Chapitre 1 - L'émergence et le développement de la transplantation d'organes en Suisse

Alexia Cochand

1.1. Les prémices de la transplantation rénale

1.2. Les premiers développements cliniques

1.3. L'émergence des transplantations hépatiques et cardiaques

1.4. Avancées cliniques 
Chapitre 2 - La création et le fonctionnement de Swisstransplant

Alexia Cochand

2.1. Mise en place des structures et définition des activités (1985-1991) 68

2.2. Mise en place de la Coordination nationale 84

2.3. Professionnalisation et anticipation des changements politiques 95

2.4. Conclusion 99

Chapitre 3 - L'organisation médicale et logistique de la transplantation: le rôle de Swisstransplant 101 Alexia Cochand

3.1. La répartition des organes avant la mise en place de la Coordination nationale 102

3.2. Garantir la compétence des centres de transplantation 117

3.3. Conclusion 130

Chapitre 4 - Les questionnements éthiques sur la transplantation dans les revues médicales (1950-1980) 133 Alexia Cochand

4.1. Les revues médicales en Suisse 135

4.2. La transplantation dans les revues: panorama chronologique 138

4.3. Les questionnements éthiques 145

4.4. Conclusion 163

Chapitre 5 - Le don et la transplantation d'organes dans la presse quotidienne: du problème biologique au problème social 167 Raphaël Hammer

5.1. La médiatisation de la transplantation entre 1945 et 1998

5.2. Les enjeux éthiques

5.3. La montée en puissance du problème de la pénurie d'organes ou la publicisation du don 
Conclusion de la partie I

Régulation de la transplantation et émergence

d'un problème public

205

Vincent Barras et Alexia Cochand

\section{Partie II.}

Le don d'organes comme enjeu de mobilisation associative

Chapitre 6 - L'espace associatif du don d'organes Raphaël Hammer et François Kaech

6.1. Délimitation de l'espace associatif du don d'organes

6.2. Quelques repères historiques

6.3. La situation contemporaine

Chapitre 7 - Une cause négligée par les pouvoirs publics Raphaël Hammer

7.1. Une cause peu reconnue

7.2. Quel modèle d'organisation pour l'échange d'organes?

7.3. Organisation hospitalière et procédures médicales

Chapitre 8 - Les enjeux de l'information et la perception du public

Raphaël Hammer

8.1. Une population a priori acquise au don d'organes

8.2. Les causes de l'indifférence et des réticences du public 
Chapitre 9-La mobilisation des associations: soutenir les malades et le don d'organes

Raphaël Hammer et François Kaech

9.1. Stratégies et activités

9.2. Rôle et pouvoir des associations

324

Conclusion de la partie II

Un espace associatif en recomposition

Raphaël Hammer

\section{Partie III.}

\section{Enjeux actuels et perspectives}

Vincent Barras, Manuel Pascual et Raphaël Hammer

Chapitre 10 - Développements du point de vue de l'Office fédéral de la santé publique (OFSP)

Andrea Arz de Falco

10.1. Aperçu

10.2. Étude relative au potentiel de la Suisse (SwissPOD)

10.3. Un rapport répondant à trois postulats

10.4. Le plan d'action 352

10.5. Organisation du projet 353

10.6. Réalisation 354

10.7. Prolongation du plan d'action 356

10.8. Perspectives

Chapitre 11 - Développements du point de vue de la fondation Swisstransplant Franz Immer

Chapitre 12 - Évolution médicale et organisation actuelle Manuel Pascual et Philippe Eckert

12.1. Travaux du Groupe des 15 en Suisse et évolution Vaud-Genève 
12.2. Loi fédérale sur la transplantation et développements en Suisse romande et au Tessin 369

12.3. Étude de cohorte suisse de transplantation (STCS) 373

12.4. Médecine hautement spécialisée en Suisse depuis 2009 374

Références 377

Sources 377

Bibliographie 380

Liste des abréviations 393 



\section{Liste des auteurs}

Andrea Arz de Falco - éthicienne, responsable de l'unité de direction Santé publique et vice-directrice de l'Office fédéral de la santé publique à Berne.

Vincent Barras - médecin et historien, directeur de l'Institut des humanités en médecine (CHUV et Faculté de biologie et de médecine), professeur ordinaire à l'Université de Lausanne.

Alexia Cochand - historienne, doctorante et chargée de recherche à l'Institut des humanités en médecine (CHUV et Faculté de biologie et de médecine, Université de Lausanne).

Philippe Eckert - directeur général et médical du CHUV à Lausanne, ancien médecin-chef de service du Service de médecine intensive adulte, ancien président du Programme latin de don d'organes (PLDO), professeur associé à l'Université de Lausanne.

Raphaël Hammer - sociologue de la santé et de la médecine, professeur à la Haute École de Santé Vaud (Lausanne), HES-SO Haute École spécialisée de Suisse occidentale.

Franz Immer - directeur de la fondation Swisstransplant à Berne, médecin spécialisé FMH en chirurgie vasculaire cardiaque et thoracique, privat-docent de l'Université de Berne.

François Kaech - sociologue et doctorant à la Faculté des sciences sociales et politiques de l'Université de Lausanne.

Manuel Pascual - médecin-chef de service du Centre de transplantation d'organes du CHUV, directeur médical du Centre universitaire romand de transplantation (CURT), professeur ordinaire et vicedoyen à la Faculté de biologie et de médecine de l'Université de Lausanne. 



\title{
Introduction. Le don et la
} transplantation d'organes dans le regard des sciences sociales et historiques

\author{
Raphaël Hammer et Vincent Barras
}

Aujourd'hui, le don et la transplantation d'organes se donnent essentiellement à voir comme des enjeux relevant de la médecine et de la santé publique. Les médias font régulièrement état de nouvelles découvertes scientifiques et techniques qui laissent augurer des avancées majeures dans le développement des greffes, de nature à répondre aux besoins des nombreux malades en attente d'un organe leur permettant d'échapper à la mort ou d'espérer un gain notable de qualité de vie. Le décalage important entre le nombre d'organes disponibles et le nombre de patients sur liste d'attente constitue un obstacle majeur dans tous les pays dotés d'une activité 
de transplantation. L'impossibilité d'offrir une thérapie éprouvée aux malades concernés constitue une exception médicale dans nos sociétés construites sur le principe d'un accès universel aux soins. Nul autre secteur de la santé n'est caractérisé par un écart aussi manifeste entre la demande et l'offre dont les conséquences sont à l'évidence dramatiques'.

Cette situation d'exception de la médecine de transplantation tient à la nature de la ressource thérapeutique en jeu et aux questions sociétales, culturelles, juridiques, politiques et philosophiques qu'elle soulève. L'utilisation de parties biologiques d'un individu, vivant ou décédé, afin de restaurer la santé d'un autre individu constitue une procédure tout à fait particulière. L'humain, fragmenté dans sa chair, élevé au rang de ressource vitale, est un fait qui figure comme une exception dans l'histoire de la médecine autant que sur le plan philosophique et social. Au-delà de ses dimensions médicales et thérapeutiques, la greffe d'organes engage des questions fondamentales et fortement chargées sur le plan symbolique et émotionnel: l'intégrité du corps, la notion de personne, l'autonomie du sujet, le statut du cadavre, la valeur des organes ou encore la définition de la mort $^{2}$. De fait, la transplantation bouleverse les frontières conventionnelles entre les corps, bouscule nos représentations ordinaires, et (ré)interroge la nature du contrat social qui lie les individus entre eux, en même temps que le rôle de l'État. Elle suscite également des débats sur l'appropriation du vivant dans le cadre des sociétés capitalistes $^{3}$, la marchandisation de la mort ${ }^{4}$, ou encore l'exploitation des plus démunis dans le trafic international d'organes humains et le tourisme de transplantation ${ }^{5}$.

1 À la fin de l'année 2019 en Suisse, 1415 patients étaient inscrits en liste d'attente pour une transplantation d'organes, dont plus de $70 \%$ attendent une greffe rénale, $14 \%$ une greffe hépatique, $6 \%$ une greffe cardiaque, $4 \%$ une greffe du pancréas et $3 \%$ une greffe pulmonaire (Swisstransplant, Preliminary statistics 2019).

2 Doniger 1996, Le Breton 2008, Bateman 2002, Lock et Nguyen 2010.

3 Lafontaine 2014.

4 Ziegler 1975.

5 Scheper-Hughes 2000. 
Si les aspects éthiques, juridiques et philosophiques sont largement documentés dans la littérature spécialisée, les enjeux de la transplantation d'organes s'inscrivent aussi dans une perspective sociologique et historique. Dans les années 1960, la greffe n'était encore qu'une technique médicale expérimentale, «une pratique ésotérique pour un petit nombre de rêveurs dans un nombre encore plus réduit de laboratoires de recherche en chirurgie ${ }^{6}$ ", relate le chirurgien pionnier Thomas Starzl. En quelques décennies, la transplantation d'organes est non seulement devenue une procédure largement pratiquée, mais aussi une question majeure de santé publique et de société. Comment, dans le contexte helvétique, la médecine de transplantation s'est-elle construite, et comment le don d'organes est-il devenu l'enjeu public que l'on connaît aujourd'hui ? Ces deux questions sont au cœur de cet ouvrage qui, sur la base de différentes sources historiques et données d'enquête obtenues sur le terrain, vise à mieux comprendre les processus clés, les acteurs, les débats et les tensions qui ont façonné et continuent de marquer la problématique du don d'organes en Suisse.

Dans cette introduction, nous exposerons les éléments de contexte qui permettent de situer l'orientation de l'étude dont l'ouvrage rend compte. Après avoir brièvement rappelé les conditions historiques et sociologiques du développement de la transplantation dans nos sociétés occidentales, nous donnerons un aperçu des principaux travaux de sciences sociales sur le don d'organes, ainsi que l'approche dite des "problèmes publics» qui a servi de trame générale à nos analyses.

\section{De l'imaginaire à la réalité contemporaine des greffes}

L'imaginaire de la transgression des frontières entre individus par le déplacement d'éléments corporels d'un être à un autre est loin d'être un phénomène récent. Le thème de l'échange d'organes ou de parties du corps est présent dans une grande variété de récits plus

6 Starzl 2003, 4. 
ou moins lointains et de cultures différentes ${ }^{7}$. Les mythologies égyptienne et gréco-latine ne sont pas en reste, à l'instar de la légende de Persée et des trois sœurs Grées devant se partager un seul œil à tour de rôle, ou d'Hermès qui réimplanta à Zeus les tendons que Typhon lui avait sectionnés. Des allusions à des greffes figurent aussi dans la Bible: ainsi, saint Antoine de Padoue qui recolle le pied d'un jeune homme et saint Pierre qui restaure les seins de sainte Agathe, amputés à la tenaille par les Romains. Mais c'est le «miracle de la jambe noire » réalisé par saint Côme et saint Damien au III ${ }^{e}$ siècle - intronisés depuis patrons des chirurgiens - qui constitue l'une des représentations les plus connues et l'une des premières - sinon la première - évocations de transplantation humaine ${ }^{8}$. Cet épisode a été largement popularisé grâce à la célèbre fresque de Fra Angelico (1387-1455) qui se diffuse en Europe dès la fin du Moyen Âge à partir de La Légende dorée de Jacques de Voragine. Tout en prenant des formes très variables, l'idée de transfert de parties du corps entre individus, dans une optique thérapeutique notamment, peut être considérée comme un mythe quasi universel ${ }^{9}$.

La concrétisation contemporaine de cette idée avec le développement des greffes de rein, de cœur, de foie et de poumon est le fruit d'une histoire non linéaire, complexe, qui se déroule sur le plan médical aussi bien que social et culturel. En effet, la transplantation s'inscrit étroitement dans l'avènement progressif de notre civilisation moderne, caractérisée par l'émergence de ce qu'il est convenu d'appeler la "raison scientifique ", et, plus particulièrement, la profonde transformation de la représentation et des modalités de connaissance du corps humain dès le $\mathrm{XVI}^{\mathrm{e}}$ siècle ${ }^{10}$. La transplantation peut ainsi être lue comme l'un des aboutissements paradigmatiques de la médecine moderne, au sens où elle constitue scientifiquement et culturellement l'emblème d'une anthropologie mécaniste ou matérielle de l'organisme humain, établie dès l'époque moderne. La

7 Küss et Bourget 1992.

8 Barkan 1996.

9 Doniger 1996.

10 Mandressi 2003. 
médecine de transplantation puise aussi dans l'imaginaire d'un idéal ancien de la réanimation des corps et d'une idéologie plus récente du triomphalisme thérapeutique ${ }^{11}$.

De manière très schématique, trois conditions historiques majeures ont déterminé le développement de la transplantation dans nos sociétés. L'accession des greffes d'organes au rang de thérapeutique quasiment routinière aujourd'hui résulte premièrement d'une histoire médicale et scientifique qui fait l'objet d'une littérature abondante. Les connaissances actuelles en matière de prélèvement et de transplantation sont le point d'aboutissement d'une longue série de tâtonnements et d'expérimentations animales et humaines qui remontent au XIX ${ }^{\mathrm{e}}$ siècle $^{12}$. Au-delà des échecs, des désillusions, des coups d'arrêt et des renoncements, l'histoire de la transplantation est caractérisée par des avancées décisives tant techniques que scientifiques dans de multiples domaines: au rang des percées capitales, on peut relever entre autres le développement de techniques chirurgicales liées à la suture vasculaire du début du $\mathrm{XX}^{\mathrm{e}}$ siècle, la compréhension des mécanismes immunitaires dans les années 1960, ou encore le développement de traitements immunosuppresseurs efficaces dans les années 1980, offrant alors la capacité non seulement de maîtriser la complexité chirurgicale des opérations de transplantation, mais aussi de surmonter le problème du rejet, resté jusque-là un obstacle majeur.

Une deuxième condition du développement de la transplantation est d'ordre institutionnel. À partir du moment où la pratique des greffes cesse d'être expérimentale et fait l'objet d'une certaine maîtrise technique par les médecins, l'enjeu majeur réside dans la mise en place d'un système de régulation et d'organisation de la transplantation, tel est le cas en premier lieu pour le rein, dès les années 1960. La reconnaissance légale du critère de la mort cérébrale, survenue pour la première fois en 1969 aux États-Unis, constitue une étape cruciale dans la mesure où cette nouvelle définition de la mort autorise juridiquement le prélèvement d'organes sur

11 Panese et Barras 2006. 
des individus qui auparavant n'étaient pas légalement décédés mais dans un état de "coma dépassé ». Le coma dépassé est alors redéfini comme étant la mort cérébrale, à savoir l'arrêt complet et irréversible de toutes les fonctions du cerveau. Jusqu'alors, seul l'arrêt cardio-respiratoire valait comme critère légal de la mort. Même si la destruction des fonctions cérébrales entraîne nécessairement dans un certain laps de temps l'arrêt du cœur et de l'ensemble des signes d'activité de l'organisme, le concept de mort cérébrale correspond à une conception nouvelle qui fait du cerveau, et non plus du cœur, le siège de la vie, ou du moins d'une certaine vie, celle retenue comme seule pertinente.

Or, pour que la transplantation d'organes devienne une activité socialement organisée, il a également fallu préciser les modalités éthiques, médicales et politiques du prélèvement et du transfert des organes. Le mode de régulation qui s'est imposé est celui du «don », par opposition au marché, signifiant que le prélèvement d'organes à des fins thérapeutiques est gratuit (le donneur n'est pas rémunéré), fondé sur le consentement (chacun est libre de déterminer le sort de ses organes et ce choix doit être respecté), et anonyme (l'identité du donneur cadavérique n'est pas communiquée au receveur). En matière de consentement au don d'organes, on distingue principalement les consentements explicite (en vigueur en Suisse) et présumé13, qui correspondent respectivement aux systèmes dits «opt-in» (engagement) et «opt-out» (retrait).

L'essor de la transplantation suppose ainsi la mise en place d'un système complexe d'institutions, de lois, règles, directives, ordonnances et protocoles qui régulent la coordination de ses différents acteurs et leurs fonctions. Nous suivrons le sociologue Philippe Steiner pour désigner du terme «continuum organisationnel» le système mis en place: un «ensemble continu d'organisations allant du signalement des personnes en situation de mort encéphalique

13 Dans le cas du consentement explicite, le prélèvement d'organes n'est autorisé que si la personne décédée avait expressément fait état du souhait de donner ses organes. Dans le cas du consentement présumé, le prélèvement d'organes est autorisé a priori, sauf si la personne décédée avait exprimé son refus de donner ses organes. Des distinctions plus fines existent, selon que le consentement est élargi ou non, définissant le degré d'implication de la famille dans la décision du prélèvement. 
dans les services de soins intensifs aux blocs opératoires des hôpitaux habilités à procéder aux transplantations, en passant par l'organisation des déplacements des malades, greffons, médicaments et équipes chirurgicales, ainsi que par les règles éthiques et techniques d'allocation des greffons aux malades inscrits sur les listes d'attente ${ }^{14}$.»

Les avancées médicales et la mise en place d'une régulation institutionnelle sont deux conditions nécessaires, mais non suffisantes. Dès lors que le don d'organes est fondé sur l'autodétermination individuelle, les activités de la médecine de transplantation dépendent étroitement de la participation du public, du «bon vouloir» des citoyens. L'adhésion des individus à la transplantation et l'acceptation du prélèvement de ses propres organes et/ou d'un proche représentent la troisième condition indispensable du développement des greffes d'organes dans nos sociétés. Elle engage un questionnement sur la manière dont le don et la transplantation d'organes "existent » sociologiquement et politiquement, sur la manière dont ces enjeux apparaissent ou sont interprétés comme un "problème » important à résoudre, comme un enjeu à valoriser. Les politiques d'information, de mise en visibilité publique, de médiatisation, les stratégies de communication, les modes d'interpellation des citoyens sont des aspects déterminants de la construction du don d'organes comme question de société. Si la pénurie de greffons représente une préoccupation de santé publique majeure (permettre un accès aussi large que possible à une ressource vitale nécessairement rare $^{15}$ ), elle est aussi la porte d'entrée de nombreux questionnements du point de vue des sciences sociales. À l'instar de tout sujet complexe, a fortiori relevant de la médecine spécialisée et qui se situe loin du champ de l'expérience ordinaire de la majorité des individus, le travail de "socialisation » du don et de la transplantation d'organes constitue un enjeu crucial.

14 Steiner 2010, 136.

15 Du fait que la mort cérébrale ne représente qu'une part infime des décès, l'identification de tous les cas de mort cérébrale suivie d'un prélèvement systématique ne suffirait pas à couvrir tous les besoins des malades en attente d'un cœur, d'un foie ou de poumon (Steiner 2010, 41). Sur la question des facteurs du manque d'organes, voir aussi Lock et Nguyen 2010, Carvais et Sasportes 2000. 
Loin d'être évident à mener et susceptible de prendre de multiples formes, ce travail de socialisation suppose d'agir sur le corps social pour faire exister la transplantation dans la société, la justifier et la faire accepter dans la population. De manière schématique, l'apport des sciences sociales, dont les premiers travaux sur la question remontent aux années $1970^{16}$, réside dans leur insistance à souligner l'importance de prendre en compte les aspects culturels et politiques du don et de la transplantation d'organes.

\section{Enjeux symboliques et résistance culturelle}

Même s'il n'est pas nécessairement en ligne de mire de leur questionnement, de nombreux travaux sociologiques et anthropologiques contribuent à éclairer le problème de la pénurie d'organes en mettant en évidence la place des enjeux symboliques dans la médecine de transplantation. L'une des idées-forces réside dans le fait que son développement est avant tout freiné par un phénomène de "résistance culturelle ${ }^{17} »$. Cette résistance n'a pas une forme socialement instituée. La transplantation en tant que telle ne fait guère l'objet de contestations organisées, ni de discours sociaux qui la remettraient foncièrement en cause ${ }^{18}$. En outre, et sans entrer dans les multiples nuances les distinguant l'une de l'autre, les principales religions (christianisme, judaïsme, islam, bouddhisme, hindouisme) ont une position plutôt favorable à l'égard du don d'organes ${ }^{19}$. En Suisse, aucun parti politique ni groupement de citoyens constitué ne milite contre la transplantation. Elle fait l'objet d'un consensus public dans nos sociétés - «il n'y a plus guère de controverse publique à propos de la moralité du don ou de la collecte d'organes ${ }^{20}$.»

16 Simmons et Simmons 1971, Fox et Swazey 1974.

17 Joralemon 1995.

18 Il existe quelques rares exceptions, à l'exemple de l'Allemagne où des groupes de citoyens expriment leur contestation de la transplantation dans l'espace public (Pfaller et al. 2018). 
Pourtant, à y regarder de plus près, «le don d'organes ne fait pas l'objet d'un consensus aussi net que ce que certains experts voudraient nous laisser entendre ${ }^{21}$ ", écrit l'anthropologue Margaret Lock, l'une des spécialistes sur le sujet. Il n'est d'ailleurs pas rare que les professionnels de la transplantation eux-mêmes évoquent une «crise de confiance» du public. La pénurie persistante d'organes peut être vue comme l'indicateur d'un travail de socialisation incomplet, le symptôme d'un «trouble» dans la société à l'égard de la transplantation ${ }^{22}$, ou le signe que le don de ses organes ou de proches n'est pas complètement entré dans les mœurs ${ }^{23}$. Pour la médecin et philosophe Anne-Marie Moulin, la «compatibilité culturelle» de la greffe a été négligée et représente l'obstacle majeur de son développement ${ }^{24}$. La résistance ou l'ambivalence face à la transplantation ne s'observe donc pas dans des discours publics ou des prises de position élaborées, mais se loge dans les interstices de l'expérience concrète du transfert d'organes.

Fondée sur une conception mécaniste du corps que l'on peut réparer (les organes comme autant de pièces détachées et remplaçables), la transplantation remet en question les significations profanes du soi, de l'intégrité du corps et les conceptions traditionnelles de la mort ${ }^{25}$. Divers travaux suggèrent que l'approche dépersonnalisée des organes propre au monde médical est mise à mal par la manière dont les donneurs vivants, les proches de donneurs et les receveurs investissent les parties du corps ${ }^{26}$. Si l'idéologie médicale occidentale promeut une vision matérialiste des organes, il n'en va pas toujours ainsi pour les patients directement concernés, pour qui «les organes transplantés représentent bien davantage que de simples "morceaux" de corps biologiques; la vie qui les anime est perçue

21 Lock 2006, 34.

22 Le Breton 2008.

23 Sharp 2006.

24 Moulin 1995.

25 Fox et Swazey 1992, Boileau 2002.

26 Sharp 1995. 
par les receveurs comme une force personnifiée qui se manifeste de surprenantes façons et module profondément leur subjectivitée ${ }^{27}$.

Dans le cas du donneur cadavérique dont l'anonymat est strictement préservé, il n'est pas rare que des patients transplantés s'interrogent sur son identité ${ }^{28}$, ou qu'ils établissent un parallèle entre leur vigueur retrouvée, leurs goûts ou intérêts et les caractéristiques imaginées du donneur, comme si le greffon conservait une empreinte de la personnalité de ce dernier ${ }^{29}$. Bien que marginaux, les fantasmes de transfert de qualités du donneur via l'organe révèlent la persistance symbolique des organes ${ }^{30}$. Si les difficultés de certains patients à intégrer le nouvel organe dans leur schéma corporel peuvent être temporaires et partie intégrante du processus de normalisation de l'identité, ces difficultés d'acceptation ont une signification culturelle plus fondamentale qui ne tient pas nécessairement au patient «mais à l'étrangeté de la situation elle-même ${ }^{31}$ ». Le vécu ambigu de la greffe révèle ainsi la dualité entre le corps-objet et le corps-sujet ${ }^{32}$. Le transfert d'organes n'est pas une opération neutre, mais une expérience qui retentit sur l'identité du receveur, et produit de nouvelles relations sociales, parfois complexes, réelles ou imaginaires, entre les donneurs ou leurs proches et ceux qui ont bénéficié de la transplantation ${ }^{33}$.

La valeur symbolique du corps se manifeste également dans le phénomène de «tyrannie du don ${ }^{34}$ », lorsque le receveur confère au don une valeur telle qu'il devient un poids psychologique et moral. Certains patients transplantés expriment alors un sentiment de

27 Lock 2006, 39.

28 Godbout 2008.

29 Boileau 2002.

30 Godbout 2008. L'impression de certains transplantés que leur personnalité a été modifiée par l'identité du donneur reçoit généralement une explication plus prosaïque: cette modification tiendrait à l'amélioration souvent notable de l'état de santé du receveur et aux effets psychotropes de certains médicaments immunosuppresseurs, à l'instar de traitements corticoïdes (Warrington et Bostwick 2006).

31 Romagnoli 2004, 195.

32 Sanner 2001.

33 Sharp 1995, Boileau 2002, Cormier, Gallo-Cruz et Beard 2017.

34 Fox et Swazey 1992. 
dette à l'égard d'un cadeau impossible à rendre, notamment dans le cadre de dons vivants ${ }^{35}$. L'attribution d'une subjectivité résiduelle à l'organe s'observe aussi chez des proches qui autorisent le prélèvement, se consolant à l'idée qu'une partie de l'être défunt "survit » quelque part ${ }^{36}$. La non-dissociation entre la personne et ses organes peut à l'inverse constituer un motif de refus du prélèvement, perçu comme à un morcellement qui porte atteinte à l'intégrité du corps du défunt ${ }^{37}$. Si elle n'alimente pas nécessairement l'ambivalence à l'égard de la transplantation, la persistance symbolique des organes témoigne d'un hiatus entre le point de vue médical et les conceptions intimes des profanes concernés. Malgré les efforts des personnels soignants pour transmettre une représentation purement biologique et fonctionnelle du greffon, celui-ci est donc loin d'être toujours envisagé comme dénué d'attache émotionnelle ou symbolique ${ }^{38}$.

\section{3. "Don" et discours publics}

Dans le prolongement de la thèse d'une résistance culturelle qui se manifeste dans la tension entre une représentation mécaniste de l'organisme et des significations fortes attachées aux parties du corps, la pénurie de greffons peut être vue comme le révélateur des insuffisances des messages d'information sur le don d'organes.

La pénurie serait ainsi pour partie le reflet de discours institutionnels qui éludent des enjeux de fond, tels que le sens éthique ultime du choix sociétal de pratiquer des greffes ${ }^{39}$. Dans le contexte français du consentement présumé, Simone Bateman critique la conception caritative de la promotion du don, qui non seulement tend à culpabiliser les non-donneurs, mais surtout «contourne toute discussion préalable sur ce qui justifie la sollicitation du don, notam-

35 Godbout (2006b) souligne que la notion de dette positive à l'égard du donneur est aussi à l'œuvre dans l'expérience de bon nombre de patients transplantés.

36 Boileau 2002.

37 Boileau 2002, Falomir-Pichastor, Berent et Pereira 2013.

38 Sharp 1995.

39 Moulin 1995. 
ment des questions sur la légitimité du besoin de parties du corps humain et les conditions dans lesquelles les uns et les autres sont appelés à participer ${ }^{40}$ ». Langlois suggère une explication similaire au manque d'adhésion d'une partie des Français au don d'organes: «Faute d'avoir pris la mesure de la valeur d'un cadavre entre le temps du décès et celui des funérailles, et pour avoir agi comme s'il était sans liens avec la sphère d'intimité, ni signification symbolique, on a indûment présupposé un consensus anthropologique sur son statut ${ }^{41}$." Des anthropologues comme Margaret Lock ou Lesley Sharp ont relevé l'ambiguïté des discours sur le don, jouant sur le double registre de la valeur sociale et morale du geste d'un côté, et de l'organe comme simple matériau biologique de l'autre: «Le don d'organes a longtemps été associé à l'altruisme, mais si tel est le cas, les parties du corps ne peuvent être considérées comme de simples objets matériels, des détritus de corps morts ou des pièces détachées de vivants mises à disposition pour le recyclage; une valeur sociale leur est inévitablement rattachée ${ }^{42}$.»

Plus largement, la rhétorique du «don» d'organes a fait l'objet de nombreuses discussions ${ }^{43}$. S'il remplit une fonction juridique en affranchissant l'acte de prélèvement de toute connotation marchande dans le lien donneur-receveur, le «don » est aussi analysé en tant que "récit culturel» (cultural account) ou idéologie. Le «don» est alors conçu comme un ensemble de valeurs et métaphores visant

40 Bateman 2002, 184.

41 Langlois $1998,67$.

42 Lock et Nguyen 2010, 252 [notre traduction].

43 La pertinence du concept de "don" dans la transplantation a été abondamment discutée à la lumière des théories anthropologiques du don, en particulier de Marcel Mauss, et des débats autour de l'utilitarisme (voir par exemple Godbout 2006a, Fixot 2010). Elle a aussi été questionnée à l'aune de la diversité des expériences vécues par les receveurs et familles de donneurs, et du sens qu'ils donnent au prélèvement et à la transplantation (Siminoff et Chillag 1999, Shaw 2015). Le terme est également contesté par Steiner pour qui le prélèvement s'apparente à "un don organisationnel dans la mesure où il est marqué par la prééminence des acteurs organisationnels sur les personnes" $(2010,238)$. D'autres travaux ont mis en avant les ambiguïtés, si ce n'est la contradiction entre l'idée de "don" et le consentement présumé, au motif que ce dernier est une "manière de fermer la bouche aux morts" (Moulin 1995, 172, voir aussi Thouvenin 1997, Lepresle 2006, Godbout et Caillé 1992). 
à légitimer socialement et favoriser des pratiques médicales qui bouleversent significativement les manières traditionnelles de penser le corps humain ${ }^{44}$. Selon Sharp, la rhétorique du "don de vie" contribue à masquer l'utilitarisme et les aspects morbides du transfert d'organes ${ }^{45}$. Portée depuis les années 1950 par le monde médical et les pouvoirs publics, la représentation dominante de la greffe en tant que «don de vie» souligne les valeurs positives d'un geste qui sauve des vies (générosité, altruisme, charité, solidarité...), et dont la grandeur morale est louée par des receveurs reconnaissants ${ }^{46}$. Or, l'évidence de la générosité dans le don d'organes n'est pas universellement perçue comme telle dans la société. Girard indique que la rhétorique de l'altruisme comme incitation au don d'organes ne correspond pas nécessairement à la grammaire ordinaire d'autres types de dons, notamment dans les milieux populaires, d'autant que la transplantation y paraît "peu constituée en objet de discours » et de représentation ${ }^{47}$.

Certains observateurs relèvent une tendance des discours institutionnels et médiatiques à présenter le don d'organes comme une évidence et à banaliser la transplantation en privilégiant un tableau positif, uniforme et consensuel sur la technique et l'acte de donner ${ }^{48}$. Les questions relatives au statut du corps et des organes, aux notions de personne et de mort font avant tout l'objet d'une lecture médicale et scientifique ${ }^{49}$. Les aspects sensibles s'avèrent peu présents dans les discours officiels - l'efficacité de la greffe, la mort cérébrale ${ }^{50}$,

44 Joralemon 1995.

45 Sharp 2006.

46 Morgan 2009, Moloney et Walker 2002.

47 Girard 2000.

48 Fortin et al. 2011, Quick, LaVoie et Stone, 2012, Feeley et Vincent III 2007, Hammer 2012.

49 Déchamp-Le Roux 1997, Le Breton 2011, Sharp 2006, Waissman 2001, Joralemon 1995.

50 La difficulté d'acceptation ou de compréhension de la mort cérébrale par les proches comme étant la mort est l'un des facteurs de refus de prélèvement, du fait de la prégnance des représentations traditionnelles de la mort cardio-respiratoire (Ralph et al. 2014, Boileau 2002). Des conceptions divergentes de la mort - «intégraliste" versus "centraliste" ou "systémiste" (Carvais et Sasportes 2000) - peuvent entrer en ligne 
l'acceptabilité de l'acte de prélèvement sur une personne décédée ${ }^{51}$-, au même titre que les aspects controversés, les difficultés ou limites de la transplantation.

Dans ce contexte, la pertinence des campagnes de sensibilisation et leur capacité à atteindre leur but sont souvent interrogées. Pour certains, la tendance des discours dominants à ignorer ou invalider les significations quotidiennes du corps et de la mort contribue aux réticences du public à l'égard des appels au don ${ }^{52}$. D'autres soulignent combien l'assimilation des attitudes de refus de prélèvement ou d'hésitation du public vis-à-vis du don d'organes à l'irrationalité, l'égoïsme ou l'ignorance ${ }^{53}$ ne résiste pas à l'examen empirique ${ }^{54}$. Ignorer ces raisons «occulterait de manière trop expéditive la complexité des phénomènes sociaux et culturels liés à cette thérapeutique ${ }^{55}$ ». La question de la reconnaissance publique de la légitimité des refus de prélèvement est également posée ${ }^{56}$. En réduisant l'enjeu du don d'organes à l'opposition entre le «oui » et le «non", les campagnes d'information ou de promotion tendent à éluder les interrogations symboliques, axiologiques et éthiques sous-jacentes à la réflexion et à la décision des individus ${ }^{57}$. Plus largement, ces différents constats suggèrent que le débat social ou la réflexion critique sur les enjeux

de compte. À noter que les difficultés de conceptualisation de la mort cérébrale se rencontrent aussi chez les professionnels de la santé (Boileau 2002, Imperatori et al. 2001). Signalons également que le critère de la mort cérébrale fait l'objet de débat en dehors de la communauté médicale (Bossi 2012). Le philosophe Hans Jonas remet en question la possibilité de connaître avec certitude la frontière entre la vie et la mort, et rejette la dualité corps-esprit qui se trouve au fondement de la mort cérébrale (voir aussi Gateau 2009, Quéré 2010, Le Breton 2008, Verspieren 2010).

51 Youngner 2003, Lock et Nguyen 2010.

52 Chavot, Felt et Masseran 2001, Chavot et Masseran 2011.

53 De nombreuses études analysent la pénurie de greffons en termes de déficit de connaissances du public, de mythes et d'idées fausses (misperception) que les campagnes d'information doivent combler ou rectifier afin d'accroître les dons d'organes (voir par exemple Sellers et al. 2018).

54 Le Breton 2008.

55 Quéré 2010,660.

56 Pfaller et al. 2018.

57 Hansen et al. 2018. 
des greffes seraient de nature à favoriser le développement d'une véritable "culture de la transplantation" dans nos sociétés ${ }^{58}$.

Le point de rencontre de nombre des travaux évoqués ci-dessus réside dans l'existence d'une opposition ou d'un décalage entre la culture biomédicale et la culture profane, qui se manifeste particulièrement sur le plan des significations et valeurs relatives à la mort et au corps. La thèse d'une résistance culturelle suggère que les discours institutionnels de justification de la greffe échouent à prendre pleinement en considération les enjeux émotionnels et symboliques qu'elle soulève, de manière plus ou moins consciente, dans une partie de la population. Dans cette optique, l'adhésion hésitante du public reflète un travail de socialisation inachevé qui pointe une carence de sens du don et de la transplantation dans la société.

\section{Aspects sociopolitiques et spécificités nationales}

Si les dimensions symboliques des greffes sont cruciales pour comprendre la complexité du don d'organes et l'expérience qu'en font les acteurs directement concernés, elles s'avèrent insuffisantes pour appréhender le don d'organes comme enjeu de société et comme produit d'un travail politique au sens large. De ce point de vue, l'analyse de "résistances" à la rationalité médicale dans d'autres domaines du champ médico-sanitaire s'avère utile, en montrant par exemple qu'il convient de ne pas réduire la question à une tension entre deux pôles, le médical et le profane, mais qu'il existe une multiplicité de points de vue, qui interdisent notamment de penser

58 Moulin 1995. L'argumentation critique des sciences sociales, de l'anthropologie en particulier, porte moins sur l'objectif thérapeutique de la transplantation que sur le plan de sa mise en œuvre dans le monde médical et des discours sociaux et moraux sur le don d'organes (Ikels 2013). Des auteurs comme Lock, Moulin ou Joralemon s'interrogent sur les moyens de mieux intégrer les greffes dans la culture. Les professionnels de santé sont également sensibles à cet aspect: "Un questionnement permanent sur les dimensions éthiques de cette pratique demeure absolument nécessaire pour le développement harmonieux de cette technique. Il est impossible d'éviter des conflits sociaux et éthiques importants si une plus grande attention et considération ne sont pas accordées aux liens entre nos techniques médicales et les valeurs de base de la société elle-même" (Alvarez 2006, 22-23). 
la culture profane comme un seul bloc homogène ${ }^{59}$. On soulignera ici en particulier que l'objet «don d'organes » est loin de se présenter de manière identique d'un pays à l'autre.

Cette diversité s'observe à différents niveaux. Sur le plan légal, c'est le consentement explicite qui est en vigueur en Suisse depuis 2007, de même que dans d'autres pays comme l'Allemagne ou les États-Unis, alors que le consentement présumé a été adopté en France en 1976 et dans plusieurs autres États européens. Au-delà des statistiques de prélèvement et de transplantation, les différences concernent également leur organisation structurelle. À cet égard, si l'Espagne présente depuis longtemps un taux de donneurs d'organes largement supérieur aux autres pays, l'explication ne réside pas dans la "nature généreuse » de ses habitants mais dans la politique et l'organisation hospitalières des greffes ${ }^{60}$. Analysant les différences régionales aux États-Unis, Healy montre que «l'altruisme» du public dépend en partie de facteurs institutionnels et de la capacité des agences d'information à susciter les dons ${ }^{61}$. Les variations de taux de donneurs relèvent moins des dispositions psychologiques plus ou moins altruistes envers son prochain que des caractéristiques des organisations en charge de promouvoir le don d'organes. La disposition au don n'est donc pas réductible à une question de culture (biomédicale versus profane) ou de représentations symboliques, mais est le produit d'une multitude d'éléments qui composent la chaîne complexe du transfert d'organes.

Il convient également de relever que les politiques nationales en matière de don d'organes peuvent être très marquées par des

59 Voir par exemple Monnais 2019.

60 Le "modèle espagnol", qui ne fait d'ailleurs pas l'unanimité dans les milieux de la transplantation, se distingue notamment par des mesures visant à maximiser le nombre de prélèvements par une dotation élevée en matière de postes de coordinateurs de transplantation, et par des incitations fortes en matière de détection des donneurs potentiels et d'obtention de l'accord des proches (Steiner 2010, 184-190). Avant l'entrée en vigueur de la loi fédérale en 2007 (LTX), le canton du Tessin affichait un taux de donneurs réels proche de celui de l'Espagne, grâce à l'engagement fort des médecins impliqués dans le processus du don d'organes.

61 Healy 2006. 
contextes sociétaux particuliers à l'exemple d'Israël ${ }^{62}$ ou de l'Allemagne ${ }^{63}$. Hogle montre que la situation en Allemagne diffère d'autres pays européens et des États-Unis en raison de la mémoire des atrocités nazies. Cette dimension historique a pesé sur l'orientation prudente des messages de promotion du don de ses organes et a contribué au choix du consentement explicite dans les années 1990. L'auteure souligne également que les médias allemands se sont souvent montrés très critiques à l'égard de la médecine de transplantation. En outre, l'Allemagne est l'un des rares pays, avec le Danemark ${ }^{64}$ et le Japon notamment ${ }^{65}$, dans lesquels la mort cérébrale a fait l'objet de débats publics nourris ${ }^{66}$. Enfin, on notera que la confiance du public à l'égard des greffes est diversement mise à l'épreuve de scandales éthiques comme la France ${ }^{67}$, l'Angleterre ou l'Allemagne ${ }^{68}$ en ont connu, alors que la Suisse a été épargnée.

Ces quelques exemples indiquent que le don et la transplantation d'organes présentent des caractéristiques spécifiques à chaque pays, façonnées par une multitude de facteurs (le contexte légal, la culture politique, l'organisation hospitalière, les stratégies institutionnelles d'information, le traitement médiatique, le développement des techniques médicales ou les sensibilités culturelles). Autrement dit, on peut avancer que l'objet «don et transplantation d'organes » a une histoire et une sociologie qui sont propres à chaque pays. Dans cette optique, nous avons pris le parti d'analyser le don et la transplantation d'organes dans le contexte helvétique en nous inspirant

62 Ben-David 2005.

63 Hogle 1999.

64 Rix 1999.

65 Margaret Lock (2002) a montré que le concept de mort cérébrale entrait en contradiction frontale avec les sensibilités de la mort et de la personne dans la culture japonaise, au point que les prélèvements sur donneurs décédés y ont été quasiment inexistants durant plusieurs décennies.

66 Pfaller et al. 2018. En Suisse, un débat public sur la mort cérébrale avait été envisagé dans le cadre des discussions parlementaires concernant la future loi sur la transplantation mais a été finalement abandonné (Perret 2010).

67 Tesnière 1993.

68 Shaw 2013. 
de l'approche dite des "problèmes publics » qui permet de prendre la pleine mesure des processus sociaux, politiques et historiques à l'œuvre dans la constitution ou le façonnement d'un enjeu de société.

\section{Le don et la transplantation d'organes en tant que "problèmes publics"}

L'approche dite des "problèmes publics ", féconde dans les sciences sociales et historiques, cherche à comprendre comment un fait social devient un problème public, c'est-à-dire une situation reconnue comme indésirable par une partie de la société, identifiée par l'État comme digne de préoccupation et comme cible légitime de l'action publique ${ }^{69}$. De manière schématique, la constitution d'un problème public fait intervenir cinq opérations possibles ${ }^{70}$. La première est caractérisée par la mobilisation d'individus qui identifient une situation qu'ils jugent anormale, inacceptable ou problématique, et qui vont s'efforcer de la faire reconnaître plus largement. Ces «entrepreneurs de cause" peuvent être un parti politique, des professionnels, des malades ou de simples citoyens qui se sentent particulièrement concernés par la situation. Dans le cas qui nous occupe, on s'interrogera notamment sur l'engagement des médecins et chirurgiens pionniers dans l'organisation institutionnelle de la transplantation, ainsi que sur le rôle des associations de patients transplantés dans la promotion de la cause du don d'organes.

Le façonnement d'un problème public suppose également «un travail de la signification " de la part des acteurs mobilisés ${ }^{71}$ qui revient à définir le problème et l'argumenter. Cette deuxième opération, de nature symbolique ou cognitive, souligne l'importance des représentations et du langage dans la manière dont un problème est qualifié ${ }^{72}$. Le terme "cadrage" désigne cette activité discursive

69 Padioleau 1982.

70 Neveu 2015b.

71 Snow 2001.

72 Rochefort et Cobb 1994, Cefaï et Trom 2001. 
de mise en sens ou de formulation d'un problème ${ }^{73}$, qui contient généralement une attribution de causalité, une évaluation des préjudices et des propositions de solutions ${ }^{\mathbf{7 4}}$. Objectivée par différents indicateurs statistiques, la pénurie de greffons, comme le montreront plusieurs chapitres de cet ouvrage, constitue le symptôme le plus manifeste du don d'organes en tant que "problème» pour différents acteurs engagés pour la cause. Plusieurs chapitres illustreront le fait que la perception d'un problème n'est évidemment pas consensuelle entre acteurs ou institutions, et que les «luttes définitionnelles ${ }^{75}$ » concernent surtout "ce qu'il faut faire » pour améliorer la situation du don d'organes, sans forcément que ces divergences soient l'objet de débat public. Par exemple, caractériser le manque d'organes comme un problème de «dons » ou de «donneurs ${ }^{76}$ " revient à cibler des responsabilités et des actions publiques très différentes.

Les chances qu'une situation accède au statut de problème public posent la question de sa justification dans l'espace public. Dans cette troisième opération, il s'agit de convaincre autrui de la gravité et de la légitimité du problème. La justification publique peut autant recourir à des critères objectifs (le nombre de personnes touchées, le coût économique...) qu'à des arguments moraux autour de valeurs centrales ${ }^{77}$. Nous montrerons que la notion de «pénurie» n'est pas la simple traduction ou objectivation d'une situation comptable défavorable à laquelle les médecins sont confrontés, et qu'elle s'est imposée comme argument dans le débat public à la faveur des discours professionnels et médiatiques.

73 Empruntée à Goffman, la notion de cadre a connu un certain succès transposé en sociologie de la communication, des médias et des mouvements sociaux, et peut être définie comme suit: "Cadrer c'est sélectionner certains aspects d'une réalité perçue et les rendre plus saillants dans un support de communication, de façon à promouvoir une définition particulière de problème, des interprétations causales, une évaluation morale ou des recommandations de traitement" (Entman in Neveu 2015b, 99).

74 Felstiner, Abel et Sarat 1991.

75 Gilbert et Henry 2009.

76 Prottas et Batten 1989.

77 Gusfield 2009. 
La quatrième opération consiste à se faire entendre et à "populariser» le problème en lui donnant une visibilité sociale large. Une cause restera confidentielle "si elle n'est pas débattue dans l'espace public, dans les institutions et lieux destinés à organiser le débat social ${ }^{78}$ ». La notion de "mise à l'agenda" décrit le fait qu'un problème donné fasse désormais partie des préoccupations, si possible durables, de la population, des médias et des responsables politiques $^{79}$. Nous montrerons que la sensibilisation du citoyen lambda et des autorités sanitaires à la cause du don d'organes a été un objectif majeur et difficile pour les associations de patients, mais aussi pour les milieux de la transplantation. La capacité à porter une cause dans l'espace public est l'un des nœuds de la compréhension de l'émergence (ou non) des problèmes publics et de leur transformation. Cette question éminemment complexe fait intervenir de multiples facteurs, tels que les ressources, stratégies et registres d'action des entrepreneurs de cause, les particularités du contexte social et institutionnel, ou encore les logiques spécifiques de fonctionnement des médias et du monde politique. Comme indiqué plus haut, le don d'organes n'est pas un enjeu de santé publique banal. Les propriétés symboliques et émotionnelles de la transplantation représentent autant une ressource qu'une contrainte du point de vue des registres de publicisation de la cause.

Dans le scénario idéal de la constitution d'un problème public, une cinquième opération est couronnée de succès: la prise en charge politique du problème, qui peut se concrétiser sur le plan législatif, économique, administratif ou encore institutionnel. Le don et la transplantation d'organes en Suisse sont aujourd'hui des problèmes publics reconnus - quand bien même certains aspects sont contestés ou débattus. Néanmoins, ce n'est que depuis peu que la médecine de transplantation et le don d'organes sont une compétence fédérale et non plus cantonale. Alors que les greffes sont pratiquées en Suisse

78 Neveu 2015b, 155.

79 Cobb et Elder 1983. 
depuis les années $1960^{80}$, c'est en 2007 qu'est entrée en vigueur la loi fédérale sur la transplantation d'organes, de tissus et de cellules (abrégée loi sur la transplantation ou LTx). La mise à l'agenda politique des greffes s'est ainsi déroulée sur plusieurs décennies. Par son ancrage "constructiviste ${ }^{81}$ ", l'importance de l'approche esquissée ici réside dans le fait qu'aucun fait social ne devient «naturellement » un problème public, par la seule force de ses caractéristiques intrinsèques, et qu'il s'agit d'un processus foncièrement contingent et social, politique et symbolique ${ }^{82}$.

C'est avec ce cadre d'analyse comme toile de fond que cet ouvrage entend rendre compte des principales dynamiques sociohistoriques et sociologiques de la constitution du don et de la transplantation d'organes en tant que problèmes publics dans le contexte helvétique, sur une période de quelque cinquante ans. Deux éclairages complémentaires de ce processus ont été choisis: la dimension temporelle et institutionnelle et la dimension associative et publique de la cause du don d'organes.

Le premier éclairage porte sur l'émergence et la consolidation des institutions de la transplantation comme pratique médicale et comme pratique sociale entre 1945 et la fin des années 1990. La période couverte correspond à cette phase cruciale de quelque quarante ans au cours de laquelle prend fin la phase expérimentale de la greffe au profit de l'établissement progressif de la phase thérapeutique normalisée que l'on connaît aujourd'hui. La littérature sur la transplantation depuis le milieu du $\mathrm{XX}^{\mathrm{e}}$ siècle relève en grande partie d'une histoire médicale internaliste qui permet de retracer le fil des avancées scientifiques et techniques dans les domaines de l'immunologie, de la chirurgie, de la réanimation, ou encore de la pharmacologie. De nombreuses autobiographies de transplanteurs pionniers, notamment américains ${ }^{83}$, donnent un peu de chair indivi-

80 La première transplantation de rein d'un donneur décédé est réalisée en 1964 à Berne et de cœur en 1969 à Zurich.

81 Hacking 2001.

82 Blumer 1971.

83 Murray 2001, Tilney 2003, Starzl 2003. 
duelle à l'essor des greffes et aux questionnements éthiques qu'elles ont suscités. Mais ces repères ne suffisent pas pour une analyse contextuelle du développement scientifique, social et politique de la transplantation et du don d'organes, qui, rappelons-le, est propre à chaque pays. En outre, les travaux historiques sur le sujet en Suisse ne sont pas légion ${ }^{84}$. Suivant une approche sociohistorique qui privilégie l'analyse des acteurs et des contextes locaux dans lesquels ils se situent ${ }^{85}$, la première partie de l'ouvrage décrit les prémices de l'organisation hospitalière et médico-sociale des activités de transplantation dans l'ensemble du territoire helvétique. Cette analyse présente l'originalité d'être fondée sur la recherche et l'exploitation d'un fonds important de différentes sources hospitalières, en particulier des archives administratives. Ces données ont été enrichies par des entretiens avec plusieurs médecins pionniers de la transplantation en Suisse, et par le dépouillement et l'analyse de corpus de presse couvrant plusieurs décennies.

Le second éclairage, qui correspond à la seconde partie de l'ouvrage, porte sur les associations de patients transplantés et leur rôle d'entrepreneurs de cause. Dans le contexte général d'affirmation des profanes face à l'institution médicale ${ }^{86}$, une vaste littérature décrit les activités et l'engagement des collectifs de patients, centrée principalement sur des fonctions psychosociales, d'une part, et de mobilisation dans l'espace public, d'autre part. C'est dans le cadre de l'augmentation des pathologies chroniques que la prise en charge des besoins des malades au sein de structures associatives a pris un essor significatif ${ }^{87}$. De tels collectifs assurent des rôles multiples, de la diffusion d'informations pratiques et scientifiques à des formes diverses de soutien aux malades, en lien notamment avec

84 On peut notamment citer les travaux de Bellanger et Steinbrecher (2006) et de Tröhler (2004) sur les débats juridico-éthiques entourant l'adoption des critères de la mort cérébrale dès 1969 par l'Académie suisse des sciences médicales (ASSM). Il faut également signaler l'étude de Hofmann (2016) qui s'intéresse, dans une optique foucaldienne, à l'émergence et à l'imposition des discours publics de la biomédecine sur le don d'organes entre 1969 et 2004.

85 Noiriel 2008.

86 Hammer 2010.

87 Herzlich et Pierret 1991, Baszanger 1986. 
l'apprentissage de l'expérience quotidienne de la maladie, le vécu identitaire ou l'intégration sociale et professionnelle. Concernant la mobilisation dans l'espace public, nombre de travaux montrent que les organisations de patients ne se contentent plus d'assumer des fonctions de coopération ou de complémentarité vis-à-vis du corps médical. Dans le sillage des mouvements de santé, notamment féministes ${ }^{88}$, les associations font preuve d'un activisme qui se déploie dans de multiples directions, en termes de lutte pour la reconnaissance officielle d'une pathologie ${ }^{89}$, de financement ou de structuration de la recherche médicale ${ }^{90}$ ou de production même du savoir scientifique ${ }^{91}$. C'est dans le contexte du sida que les malades ont incarné « une réelle force sociale de changement ${ }^{92}$ ». En France ${ }^{93}$ et aux États-Unis ${ }^{94}$, la forte mobilisation collective des malades du sida leur a permis d'acquérir une légitimité auprès des instances publiques au point d'infléchir les politiques sanitaires et de modifier les procédures d'expérimentation de nouveaux traitements. L'idée d'un nouveau pouvoir collectif des patients doit cependant être tempérée. Premièrement, parce que la construction efficace d'une cause ne va pas de soi et dépend de toute une série de facteurs, dont la capacité des associations à établir un front commun ou à coopérer ${ }^{95}$, et à trouver la bonne distance critique vis-à-vis des autorités ${ }^{96}$. Deuxièmement, parce que le contexte joue un rôle déterminant. L'activisme exemplaire des malades du sida a beaucoup tenu à une conjoncture médicale, épidémiologique et sociologique tout à fait

\footnotetext{
88 Löwy 2005.

89 Fox 1989, Loriol 2003, Ngatcha-Ribert 2012, Chamak 2008.

90 Panofsky 2010.

91 Rabeharisoa et Callon 1999.

92 Carricaburu et Ménoret 2004, 162.

93 Barbot 2002.

94 Epstein 2001.

95 Bach 1995, Wood 2000.

96 Akrich, Méadel et Rabeharisoa 2009.
} 
spécifique, donnant à cette mobilisation un caractère à maints égards plus exceptionnel que typique ${ }^{97}$.

Dans ce paysage contrasté, comment se situent les associations de patients transplantés ${ }^{98}$ ? Leurs buts sont-ils plutôt orientés vers l'entraide ou vers une mobilisation dans la sphère politique et médicale? Quelle est leur influence sur la promotion du don d'organes comme problème public? Pour apporter des réponses à ces questions, nous avons mené une enquête sociologique qualitative en Suisse romande afin de comprendre la dynamique d'engagement des associations par rapport à la greffe comme enjeu de santé et surtout comme cause publique. Deux aspects ont plus particulièrement retenu notre attention: les cadres de perception du don d'organes en tant que problème, et les activités concrètement réalisées et leur signification du point de vue des collectifs de transplantés.

\section{Structure de l'ouvrage}

Dans la première partie de l'ouvrage qui privilégie la perspective historique, le chapitre 1 exploite la documentation administrative produite par les hôpitaux suisses pour décrire le développement clinique et institutionnel de la transplantation du rein à partir des années 1950, puis du cœur et du foie. Il met en avant le rôle clé qu'ont joué certains médecins pionniers, et l'influence du mode de fonctionnement hospitalier dans lequel la profession médicale jouissait d'une forte autonomie. Le chapitre 2 retrace l'émergence dans les années 1980 de Swisstransplant comme acteur central du don et de la transplantation d'organes, en s'appuyant sur les procès-verbaux des structures constitutives de la fondation. Cette analyse expose les prémices de la création de Swisstransplant (les groupes de travail de médecins), son fonctionnement interne et structurel, et ses objectifs

97 Barbot 2002, Dodier 2003.

98 Contrairement à des thèmes comme le sida ou le cancer, les associations de patients en lien avec la transplantation et le don d'organes n'ont pratiquement pas retenu l'attention des chercheurs en sciences sociales, à l'exception pour la Suisse de Hofmann (2016) qui y consacre plusieurs pages, et de Biaudet (2013) et Plumart (2012) pour la France. 
dans le contexte de son institutionnalisation progressive jusqu'à la fin des années 1990. Le chapitre 3 prolonge les analyses précédentes en examinant spécifiquement la régulation des échanges d'organes entre les différents centres de prélèvement et de transplantation, sous l'angle à la fois organisationnel, professionnel, éthique et politique. Les deux chapitres suivants abordent la transition des greffes comme procédure expérimentale vers une procédure thérapeutique à part entière sous un angle différent, à travers son écho dans deux types de presses (professionnelle et grand public). Sur la base d'articles de revues médicales (1950-1980), le chapitre 4 détaille les enjeux scientifiques et les questionnements éthiques qui sont diffusés à la communauté des médecins non spécialisés dans le domaine. Partant de la couverture médiatique sur quelque cinquante ans d'éditions du Journal de Genève, le chapitre 5 montre comment le don et la transplantation d'organes se sont développés comme sujets d'intérêt public, par le biais notamment de l'importance croissante dans les années 1980 et 1990 des aspects politico-juridiques et de la pénurie d'organes.

Dédiée au rôle joué par les associations de patients transplantés, la seconde partie de l'ouvrage est composée de quatre chapitres qui s'appuient principalement sur des entretiens auprès de responsables et membres de différents groupements de patients en Suisse romande. Le chapitre 6 décrit les caractéristiques majeures de ce que l'on appellera l'espace associatif du don d'organes romand, du point de vue de son émergence historique, sa composition, ses ressources et ses objectifs. La présentation des questions de recherche et des aspects méthodologiques de l'étude complète ce chapitre. Les deux chapitres suivants sont consacrés au point de vue des acteurs associatifs sur la situation du don d'organes en Suisse dans le contexte des années 2012-2014, soit peu de temps après l'entrée en vigueur de la LTx. Cette analyse identifie trois manières de percevoir le don d'organes: comme problème politico-législatif, questionnant l'orientation de la LTx et des campagnes publiques officielles, et comme problème hospitalier, en référence au manque de ressources dans les hôpitaux et aux attitudes de certains professionnels directement impliqués dans le processus du don d'organes (chapitre 7). 
La perception du don d'organes comme un problème d'information de la population est l'objet du chapitre 8 . Il examine comment les acteurs associatifs interprètent les attitudes favorables et défavorables de la population générale vis-à-vis du don d'organes, ainsi que le rôle qu'ils attribuent à l'information du public pour améliorer la situation. Le chapitre 9 décrit les stratégies et les activités concrètes réalisées par les associations, et interroge leur influence sur le don d'organes comme problème public, ainsi que les difficultés d'une mobilisation forte et collective autour de cette cause. Ce chapitre est suivi de quelques pistes de réflexion sur le rôle joué par l'espace associatif au cours des dernières années et sa configuration possible dans le futur.

Pour compléter les analyses sociohistorique et sociologique des deux parties académiques de l'ouvrage, une dernière partie «Enjeux actuels et perspectives " donne la parole à des représentants des principaux acteurs de la transplantation d'organes en Suisse. Nous remercions en particulier la Dr Andrea Arz de Falco (OFSP), le Dr Franz Immer (Swisstransplant), ainsi que les Prs Philippe Eckert et Manuel Pascual (CHUV et université de Lausanne) d'avoir accepté d'exposer la situation actuelle et à moyen terme du don et de la transplantation d'organes. L'analyse sociologique ayant en quelque sorte relayé la "voix » - souvent critique - du monde associatif, la dernière partie permet de compléter l'éventail des points de vue. Surtout, elle informe le lecteur des changements médicaux, institutionnels et organisationnels récents intervenus depuis l'enquête de terrain menée auprès des acteurs associatifs, et permet de connaître les perspectives politiques et médicales du développement de la transplantation dans un proche avenir. En particulier, la question importante du type de consentement, présumé ou explicite, figurera au cœur de l'agenda politique, et sera susceptible de modifier significativement la configuration du don d'organes comme problème public. Au total, par leur complémentarité et leur diversité, les différents chapitres de cet ouvrage permettent de couvrir des enjeux historiques et sociopolitiques importants de la transplantation en Suisse sur une période qui s'étend de 1945 à 2020. 
Partie I.

L'émergence

institutionnelle

et publique

du don et de la

transplantation

d'organes 



\section{Chapitre 1}

\section{L'émergence et le développement de la transplantation d'organes en Suisse}

\section{Alexia Cochand}

La transplantation d'organes, caractérisée notamment par l'intervention de médecins aux spécialisations distinctes et par le recours à des infrastructures médico-techniques de pointe, est intrinsèquement liée à l'institution hospitalière. Dans un espace de développement fragmenté, constitué par les cinq hôpitaux universitaires actuels - Zurich, Berne, Lausanne, Genève et Bâle - et l'hôpital cantonal de Saint-Gall, la mise en œuvre de ces pratiques s'effectue par organe en deux phases. Les premiers programmes de transplantation au milieu des années 1960 concernent le rein et s'étendent par la suite au foie et au cœur dans les années 1980 grâce à la découverte de la cyclosporine. L'extension de ce domaine de la médecine à l'ensemble des organes solides se concrétise progressivement par la mise en place de programmes de transplantation plus marginaux dès la fin des années 1980 (pancréas, poumon, intestin). Largement influencé par le contexte médico-scientifique international, ce développement est néanmoins façonné dans des établissements hospitaliers indépendants les uns des autres, marqués par une organisation administrative et une répartition des compé- 
tences de gestion variées. Aussi, bien que la valeur thérapeutique représente incontestablement un motif qui explique l'ajout de la transplantation à l'offre médicale des hôpitaux, l'influence d'enjeux médicaux, sociaux et économiques configure la mise en place de ces pratiques.

C'est donc entre variabilité des contextes de gestion, spécificités techniques des types de transplantations et rôle d'acteurs locaux que nous proposons de saisir dans ce chapitre le développement de ces pratiques dans les hôpitaux suisses. Pour évaluer et mesurer le rôle des systèmes de gestion administrative des hôpitaux sur le processus de développement des différents types de transplantations, nous suivrons l'apparition de ces pratiques par organe. Nous nous intéresserons d'abord à la transplantation rénale, puis traiterons simultanément de la mise en place des transplantations hépatiques et cardiaques. Bien que non immédiatement liées sur le plan médicotechnique, ces deux pratiques sont l'objet de discussions et réflexions conjointes en raison de la proximité chronologique de leur mise en œuvre.

Il s'agit pour ces trois organes d'examiner d'abord la période d'émergence, avant de retracer le processus de mise en place de la pratique à l'offre thérapeutique de l'établissement. La mise en évidence du rôle joué par les différents acteurs et l'identification des intérêts professionnels qu'ils défendent représentent un aspect central. Nous sommes également particulièrement attentifs aux rapports interprofessionnels qu'entretiennent les médecins et le personnel administratif et aux moyens mobilisés pour exprimer leurs intérêts particuliers. Enfin, nous soulignerons, dans une perspective comparative, les spécificités des développements de la transplantation rénale, hépatique et cardiaque.

Ce chapitre s'appuie sur la documentation administrative produite par les établissements universitaires de Genève, Lausanne, Berne, Bâle et Zurich, et de l'hôpital cantonal de Saint-Gall pour la période comprise entre 1950 et 1990 . Plus précisément, les documents analysés prennent des formes hétéroclites (courriers, procès-verbaux de conseil de direction ou encore rapports d'activité). L'état de conservation de ces sources varie d'un hôpital à l'autre: 
Berne, Lausanne et Genève présentent la documentation la plus abondamment préservée, alors qu'elle est plus fragmentaire à Bâle et Saint-Gall ${ }^{99}$. La production documentaire administrative de l'hôpital de Zurich n'a à notre connaissance pas été conservée ni sur place ni dans les archives du canton. Nous avons pu pallier en partie cette répartition inégale des sources dans les archives hospitalières grâce à l'exploitation du corpus de revues médicales suisses couvrant les années 1960 à 1990, analysé spécifiquement dans le chapitre 4 . Souvent écrits par les transplanteurs eux-mêmes, les articles de ces revues permettent en effet d'identifier le contexte international dans lequel les transplantations se développent, de saisir le regard porté sur ces pratiques par les médecins helvétiques ainsi que de suivre l'évolution des résultats obtenus dans les hôpitaux suisses. Ce chapitre prend également appui sur des entretiens menés avec quatre médecins, liés au domaine de la transplantation, en activité lors du développement de la transplantation en Suisse. Enfin, nous avons également survolé à titre exploratoire les bulletins des séances du Grand Conseil du canton de Genève afin de suivre, le cas échéant, le rôle des instances politiques dans le développement de la transplantation.

\subsection{Les prémices de la transplantation rénale}

Sur la base de rétrospectives établies par les centres eux-mêmes ${ }^{100}$ ou par l'Office fédéral de la santé publique ${ }^{101}$ (OFSP), on peut situer la réalisation des premières interventions menées dans chacun des hôpitaux dans un cadre chronologique sommaire. Les premières transplantations de rein en Suisse sont réalisées d'abord à Berne,

99 Sur la conservation des archives administratives des hôpitaux universitaires suisses, voir Cochand 2017.

100 Voir par exemple le site du Centre de transplantation d'organes (CTO) du CHUV: www. chuv.ch/transplantation/cto_home/cto-le-centre-en-bref/cto-le-cto-en-bref-historique.htm (consulté en 2019).

101 Voir par exemple la page internet du site de l'OFSP, "La médecine de transplantation: un long passé": www.bag.admin.ch/bag/fr/home/medizin-und-forschung/transplantationsmedizin/transplantieren-von-organen-geweben-Zellen/geschichte-dertransplantation.html (consulté en 2019). 
puis à Zurich en 1964. Après une coupure de quelques années, elles se développent selon un processus de diffusion plus général et temporellement plus rapproché: elles sont effectuées en 1968 et 1969 à Bâle et Saint-Gall avant de s'étendre en Suisse romande, où elles sont pratiquées à Genève dès 1970 et à Lausanne dès 1971.

Si la réalisation de ces "premières » témoigne de l'existence d'un savoir médico-technique dans chaque centre, l'émergence et la mise en place de ce savoir restent difficiles à retracer. Si les aléas de la conservation des sources jouent un rôle, la rareté de la documentation directe pour cette période des années 1960-1970 s'explique surtout par une gestion administrative des pratiques médicales principalement assumée par les médecins eux-mêmes. Entre pairs et sans l'implication marquée d'acteurs extérieurs à la sphère médicale, la mise par écrit, rendue nécessaire par la rédaction de rapports, de budget ou présentation d'objectifs médicaux, est marginale ${ }^{102}$. Ainsi c'est surtout à la faveur de processus informels, comme des discussions et arrangements entre médecins, que la transplantation rénale est organisée dans les hôpitaux. La reconstitution de son développement est donc le plus souvent réalisée en "creux", par déduction face aux silences de la documentation ${ }^{103}$.

\section{Circulation des savoirs}

Dans une phase dont l'inscription chronologique précède les premières interventions thérapeutiques, la transplantation rénale fait l'objet d'expérimentations par un nombre limité de médecins. Quelques rares mentions indirectes attestent d'une période où s'élabore l'acquisition d'un savoir spécifique, en marge de l'activité thérapeutique habituelle. À Saint-Gall, le président du Collège des chefs de service annonce en 1968 dans le rapport annuel fourni par

102 Sur le contexte économique favorable dans lequel les services médicaux se multiplient au sein des hôpitaux, voir Donzé 2003, 231-ss.

103 Comme cela est attesté sur le plan international, le chirurgien transplanteur est une figure centrale de cette phase expérimentale du fait de son rôle clé dans l'implantation de nouvelles techniques et la diffusion d'innovations scientifiques (Steiner 2010). Cette phase historique est marquée du sceau de l'espoir, qui résume la tension entre l'incertitude thérapeutique et l'ethos médical qui pousse à tenter quelque chose pour soulager la détresse des malades (Steiner 2010, Fox et Swazey 1974). 
l'hôpital cantonal que, grâce "à un travail expérimental et à l'étude intensive des questions théoriques ${ }^{104}$ ", la clinique de chirurgie se prépare à la réalisation de transplantation dans l'année à venir. À Genève, c'est le travail entrepris "ces huit dernières années ${ }^{105}$ » qui permet, comme le relève René Mégevand de la Clinique universitaire de chirurgie, la première transplantation rénale le 14 novembre 1970 .

Cette phase expérimentale consiste vraisemblablement en l'adaptation de pratiques et techniques mises au point dans les centres de référence à l'étranger. À cet égard, les voyages "d'études » représentent un moyen d'acquisition de connaissances médico-techniques en lien avec la transplantation dont disposent les médecins suisses. Gilbert Thiel, néphrologue de l'hôpital de Bâle, s'est ainsi formé dans le centre dirigé par John Merrill, lequel a dirigé la première transplantation rénale entre jumeaux homozygotes en 1954 à Boston ${ }^{106}$. Fondé sur des facteurs hétéroclites renvoyant à des trajectoires personnelles, l'intérêt scientifique développé par ces praticiens pour la transplantation représente un préalable indispensable à son émergence dans les hôpitaux suisses.

À leur retour de l'étranger, certains chirurgiens mettent en place dans un délai variable des programmes expérimentaux autour desquels se constitue peu à peu une «équipe ». Le silence documentaire qui entoure cette période renforce l'image d'activités menées en marge de la pratique médicale proposée par les établissements. Le passage de l'expérimentation animale aux premières tentatives d'application clinique sur l'homme est difficile à identifier chronologiquement. Les échecs médicaux que représentent ces transplantations sporadiques contribuent certainement à maintenir la transplantation aux marges des activités médicales jusqu'à la fin des années 1960 . À Berne par exemple, ce sont probablement les quelques tentatives infructueuses effectuées en 1964 qui poussent les médecins à aban-

104 Staatsarchiv St-Gallen, Jahresbericht über die Verwaltung des Santitätswsens des Kantons St.Gallen im Jahre 1968, Zweiter Teil: Krankenanstalten, p. 26 [notre traduction].

105 Mégevand 1972, 982.

106 www.living-donor-care.ch/pages/de/prof.-gil-thiel.php (consulté en 2019). 
donner l'expérimentation clinique. À cet égard, l'hôpital de Zurich, où en 1966 on dénombre déjà seize transplantations dont certaines permettent à des patients de survivre vingt et un mois, constitue une exception dans le contexte helvétique ${ }^{107}$.

\section{La transformation des centres de dialyse}

Depuis le début des années 1950, le perfectionnement progressif des appareils de dialyse en fait un élément essentiel dans la prise en charge des patients souffrant d'insuffisance rénale irréversible. Si la dialyse conduit à un prolongement de la durée de survie des patients, elle entraîne une surcharge des espaces où est proposé ce type de traitement. Un mélange de facteurs économiques et logistiques, comme le coût des appareils, l'implication d'un personnel soignant nombreux ou encore le manque de locaux, est avancé par les médecins pour expliquer la stagnation du nombre de lits attribués à ces unités. Le manque de places nécessite dès lors une sélection des patients qui met les néphrologues devant une situation délicate, comme l'évoque Claude Perret de la clinique universitaire de médecine de Lausanne dans la leçon inaugurale qu'il tient en 1966:

"Pour de multiples raisons d'ordre médical, économique ou financier, il est impossible actuellement de recourir au procédé de la dialyse à long terme chez tous les malades en insuffisance rénale chronique terminale. Cela suppose un choix de la part du médecin qui décidera finalement de la vie du malade. Quels critères adopter pour établir cette sélection? Quels arguments le médecin fournirat-il pour justifier sa décision ${ }^{108}$ ? »

À cet égard, les critères médicaux engageant le pronostic vital à court terme ne représentent plus pour le patient la garantie d'obtenir une place dans un centre de dialyse. D'autres conditions complémentaires sont nécessaires pour faire face à la demande accrue de prise en charge. Un néphrologue actif dans un centre de dialyse au cours

107 Filippini 1967, 768.

108 Perret 1966, 1510-1514. 
de sa carrière explique dans un entretien que dans le centre où il exerçait "les patients de plus de 50 ans étaient refusés ${ }^{109}$ ».

Ce contexte sanitaire contribue certainement à favoriser le développement des transplantations dès le milieu des années 1960 dans les hôpitaux aux ressources financières et logistiques suffisantes. Sous réserve d'une étude complémentaire de l'activité des centres de dialyse des hôpitaux de Berne et de Zurich à cette période, on peut supposer que c'est dans ce contexte que les premières transplantations y sont menées au milieu des années 1960.

Avec la généralisation des tests de compatibilité cellulaire entre donneur et receveur, les centres de dialyse deviennent peu à peu, à partir du milieu des années 1960, essentiellement destinés aux patients dans l'attente d'une transplantation. Rassemblés, ils représentent un "pool» dans lequel le receveur le plus compatible est choisi lorsqu'un organe est disponible. L'élaboration de programmes de dialyse à domicile, dont l'émergence se situe en Suisse au début des années $1970{ }^{110}$, contribue à la transformation des centres de dialyse des hôpitaux universitaires en lieux de soins transitoires. On le constate par exemple à Genève, où, pour expliquer l'absence de programme de transplantation, le Conseil d'État en réponse à une interpellation explique qu'«il était tout d'abord nécessaire d'avoir un centre de dialyse chronique traitant les malades candidats à la transplantation ${ }^{111}$ ».

\section{Des perspectives prometteuses}

Au sein du corps médical large, la transplantation rénale constitue vraisemblablement au milieu des années 1960 une pratique limitée à la connaissance de praticiens spécialisés. Dans la revue genevoise Médecine et Hygiène (MÆH), les premières transplantations rénales des années 1950, comme celles du début des années 1960, représentent un fait médical porteur d'espoir sans

109 Entretien réalisé le 18 novembre 2015.

110 Sur le développement de la dialyse à domicile en Suisse, voir par exemple Descoeudres

C., "L'hémodialyse à domicile", Revue médicale de la Suisse romande, 6, 1973, 441-450.

111 Archives de l'État de Genève, Mémorial du Grand Conseil, 1969, p. 1329. 
toutefois constituer la une de la revue. Alors que les aspects techniques comme le typage de tissus cellulaires et la mise en place de nouveaux protocoles médicamenteux favorisent l'obtention de meilleurs résultats, la transplantation est perçue, peu à peu, comme une option dans le traitement des insuffisants rénaux terminaux. Dès la fin des années 1960, elle devient une opération dotée d'une valeur thérapeutique incontestable pour le public médical large. Elle apparaît en 1972 dans le classement des interventions les plus marquantes figurant dans l'édition spéciale publiée à l'occasion du millième numéro de la revue ${ }^{112}$.

La médiatisation de la première transplantation cardiaque en décembre 1967 en Afrique du Sud entraîne certainement l'émergence d'un intérêt pour l'ensemble de ces pratiques hors de la sphère médicale ${ }^{113}$. Quatre mois après cette première mondiale, un député genevois interpelle le Conseil d'État au sujet de l'opportunité de développer la transplantation rénale. Cette requête est justifiée pour son auteur par «[...] les progrès des techniques de transplantation rénale et leurs résultats de plus en plus favorables ${ }^{114}$ ».

\subsection{Les premiers développements cliniques}

La transplantation rénale se développe essentiellement dès la fin des années 1960 entre spécialistes, en marge des processus de gestion administrative: la rédaction de rapports sur des faits médicaux comme la réalisation des premières transplantations, leur fréquence, ou encore la prise en charge des patients au sein de l'établissement reste marginale sans constituer un moyen de contrôle des activités ou de diffusion d'information. La création par les médecins de groupes de travail indépendants des hôpitaux, où siègent des représentants de chaque centre, accroît la difficulté à retracer le processus d'organisation de la transplantation. À la fin des années 1960, les

$112 \mathrm{M} \& H, 1^{\mathrm{er}} \operatorname{mars} 1972,325$.

113 Nathoo 2009. Comme en témoigne aussi l'importante couverture médiatique dans la presse écrite (voir chapitre 5).

114 Archives de l'État de Genève, Mémorial du Grand Conseil, 1969, p. 1329. 
questions scientifiques et logistiques liées à la transplantation sont traitées au sein de trois espaces distincts: le groupe pour l'histocompatibilité en 1968, celui pour la chirurgie de transplantation en 1969, auxquels s'ajoute la commission pour la transplantation rénale fondée au sein de la Société suisse de néphrologie ${ }^{115}$. Lors de ces réunions, les spécialistes de différents établissements mettent sur pied l'organisation de la transplantation et l'adaptation de sa pratique sans véritablement prendre en considération les contextes de gestion et de fonctionnement des hôpitaux.

La large implication de groupes de médecins représente une caractéristique commune aux réflexions entreprises dans les différents hôpitaux autour de la mise en œuvre de la transplantation d'organes. La mainmise des praticiens sur l'organisation de la transplantation se constitue à la faveur d'un lien implicite entre l'exercice d'une activité médicale et l'organisation logistique. À Berne, c'est lors d'une séance du Collège des médecins en février 1968 que le développement de la transplantation est abordé. Le chirurgien Wälti y soumet une requête concernant la création d'un groupe de travail afin d'organiser la réalisation de transplantations ${ }^{116}$. Fort des compétences qui lui sont reconnues au sein de l'établissement, il se voit confier la mise sur pied, avec André Montandon, d'un groupe de travail. Autorisés à "prendre en main tout le travail jugé nécessaire $^{117}$ », les deux médecins consacrent une partie de leur temps au développement de la transplantation. Les activités de ce groupe, composé probablement de médecins aux spécialisations variées comme des immunologues, des chirurgiens ou des neurologues, conduisent à la constitution d'un programme de transplantation dès 1969 à Berne.

À Genève, les Prs René Mégevand, Alex Müller, Jean-Claude Rudler et René Mach prennent officiellement en main la mise en place d'un programme de transplantation dès juillet 1968. Actifs

115 Voir chapitre 2.

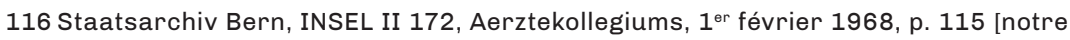
traduction].

117 Idem, p. 116 [notre traduction]. 
dans les cliniques universitaires de médecine, de médecine thérapeutique et de chirurgie, ils y sont sans doute tacitement autorisés en raison de leur statut hiérarchique et de leur expertise scientifique. Comme Mach le rapporte dans un courrier, ils se rencontrent informellement: «[...] le lundi 28 juillet 1969, les Prs Mégevand, Müller, Rudler et Mach se sont réunis dans mon bureau pour mettre au point l'organisation de la transplantation rénale ${ }^{118}$.» Les besoins matériels, humains et logistiques que nécessite l'ajout de la transplantation à l'offre thérapeutique de l'établissement sont sommairement établis durant cette réunion et transmis à la direction. Un appareil de dialyse, la réfection d'un local et l'engagement d'infirmières sont demandés pour permettre la mise en route du programme, sous réserve d'une réévaluation ultérieure ${ }^{119}$. La mise en place de programmes de transplantation requiert une collaboration accrue de plusieurs spécialités médicales, à l'instar des immunologues dont la contribution devient essentielle. À cet égard, Michel Jeannet, rentré à Genève après un séjour à Boston où il se consacre au développement de méthodes de typage cellulaire, y contribue très probablement par son activité au sein du laboratoire d'immunologie de l'hôpital dès 1967.

Si les médecins bénéficient d'une légitimité incontestable lorsqu'il s'agit de définir l'orientation des pratiques médicales, leur marge de manœuvre est déterminée par le contexte sanitaire et politique de chaque établissement. À Genève toujours, la particularité de la configuration hospitalière représente plutôt un frein. En août 1970, comme l'explique le directeur à Mach, qui invoque le retard pris sur les autres établissements, la situation à laquelle doit faire face l'hôpital cantonal est particulière:

«[...] Il n’y a aucune commune mesure entre l'Hôpital universitaire de Genève et ceux de Bâle, Berne et Zurich. À Genève, nous sommes obligés de prendre tous les cas qui nous sont envoyés aigus ou bénins. Dans les Hôpitaux universitaires de Bâle, Berne et Zurich, on a le loisir de ne prendre que les cas aigus et de refuser

118 HUG, AC, 501, transplantation, lettre du Pr Mach au directeur M. Haas, 4 août 1969. 119 Idem. 
les autres. Il y a suffisamment d'hôpitaux de dégagement dans ces cantons pour le faire ${ }^{120}$.»

Finalement, la première transplantation rénale à Genève est effectuée le 14 novembre 1971 sans que les questions liées à l'organisation ne soient plus abordées, par écrit du moins.

\subsection{L'émergence des transplantations hépatiques et cardiaques}

Inscrite dans le contexte de gestion économique des hôpitaux de la fin des années 1980 caractérisé par l'omniprésence des impératifs budgétaires, la mise en place des transplantations hépatiques et cardiaques est marquée par un contrôle accru des dépenses. La répartition du personnel, l'occupation des locaux ou encore la création de postes sont désormais évaluées et régulées selon des critères financiers prépondérants ${ }^{\mathbf{1 2 1}}$. Aux côtés des médecins, les commissions administratives et les autorités politiques cantonales prennent activement part au processus de développement clinique de types de transplantations inédites.

\section{Phase expérimentale}

De manière similaire à la transplantation rénale, l'émergence des transplantations hépatiques et cardiaques est liée aux expérimentations menées sous l'impulsion de médecins au bénéfice d'une expertise dans ce domaine. Selon le récit qu'il en fait rétrospectivement, Adrien Rohner, en activité à la clinique de chirurgie digestive de Genève, se rend dès 1970 à Denver dans l'unité de référence dirigée par le pionnier Thomas Starzl «pour apprendre la transplantation hépatique expérimentale ${ }^{122} »$. Après ce séjour dont on ignore la durée, il rentre à Genève où il continue de pratiquer régulièrement cette technique en laboratoire, durant deux ans. Si son récit laisse

120 HUG, AC, 501, transplantation, lettre du directeur Haas au Pr Mach, 20 août 1970.

121 Donzé 2003, 291-ss.

122 HUG, AC, 0037, transplantation, foie, Projet, transplantation hépatique humaine, 10 février 1986, p. 1. 
bon nombre de questions organisationnelles en suspens, il met en évidence le lien de dépendance entre les centres "pionniers» étrangers et le reste des hôpitaux sur le plan de la transmission des connaissances dans le domaine de la transplantation au début des années 1970. Dans un contexte médico-scientifique où la communauté médicale fonde de grands espoirs sur un développement thérapeutique rapide, les rares centres au bénéfice d'infrastructures spécialement consacrées à la réalisation de ces pratiques et de soignants expressément formés représentent un point de chute pour les spécialistes étrangers.

Les éléments biographiques, bien que lacunaires, montrent que l'intérêt scientifique et personnel des praticiens pour la transplantation s'explique à la lumière de leurs parcours individuels. Confrontation fortuite au cours d'un séjour professionnel ou volonté affirmée de se familiariser avec une pratique médicale inédite, chaque parcours fait écho à un cheminement spécifique. À Zurich, où l'activité liée à cette technique est la plus avancée en Suisse, la transplantation est développée grâce à l'expérience acquise par Åke Senning, chirurgien cardiaque suédois à la tête du Département de chirurgie depuis sa création en $1960^{123}$. Actif dans le domaine de la transplantation rénale dès le début des années 1960, il réalise, avec son équipe, la première transplantation cardiaque de Suisse en 1969. Dès 1965, il est rejoint par Felix Largiadèr, de retour d'un séjour à Minneapolis durant lequel il met au point une procédure inédite de transplantation pancréatique ${ }^{124}$.

En Suisse, les activités expérimentales, quel que soit l'organe, gardent toutefois un caractère éminemment marginal durant cette période, en raison des difficultés liées à son application clinique à court ou moyen terme. Rohner explique en effet qu'à Genève «les résultats de la transplantation hépatique demeurant nettement en retrait par rapport à ceux de la transplantation rénale, l'effort entre-

123 Il implante notamment le premier pacemaker permanent en 1958. Sur son activité, voir www.vis.usz.ch/ueber-die-klinik/geschichte/seiten/ake-senning.aspx (consulté en 2019).

124 Sur le parcours de Largiadèr, voir www.vis.usz.ch/ueber-die-klinik/geschichte/ seiten/felix-largiader.aspx (consulté en 2019). 
pris initialement $[\ldots]$ a été détourné momentanément vers d'autres secteurs ${ }^{125}$ ». En effet, dans le cas des transplantations hépatiques au début des années 1970, le taux de survie à un an oscille entre $20 \%$ et $30 \%$.

Cette mise entre parenthèses des activités expérimentales met en évidence la prédominance de la mission sanitaire qui gouverne les hôpitaux publics: l'implication de praticiens dans les programmes de recherche et la mise à disposition de ressources matérielles sont influencées par la perspective d'un développement clinique dans un délai proche. Le perfectionnement de la procédure opératoire, l'expérimentation de nouveaux protocoles médicamenteux ou l'affinement des critères de sélection des donneurs et receveurs permettant une survie prolongée sont progressivement attribués à quelques centres spécialisés. À cet égard, la première transplantation cardiaque réalisée à Zurich en 1969 s'inscrit dans la vague d'enthousiasme international d'une partie des médecins après l'intervention menée au Cap en 1967: en 1968, plus de 115 transplantations sont effectuées à travers le monde selon les chiffres évoqués par le corps médical127. À la même période, Hossein Sadeghi, responsable du département de cardiologie des services universitaires de médecine et de chirurgie de Lausanne, indique à propos de la transplantation cardiaque que l'équipe du service de chirurgie est en effet "préparée à de telles interventions ${ }^{\mathbf{1 2 8}}$ ». Le problème réside dans les conditions de prélèvement que les pouvoirs publics doivent encore codifier.

Les controverses éthiques suscitées par la "première » à Zurich ${ }^{129}$, auxquelles s'ajoutent les difficultés habituellement rencontrées liées à la gestion du rejet, mettent un terme à l'activité expérimentale et clinique dans ce domaine en Suisse. L'absence d'allusion même

125 HUG, AC, 0037, transplantation, foie, Projet, transplantation hépatique humaine, 10 février 1986, p. 3.

126 Mosimann 1992, 121.

127 Goy 1993, 417.

128 Société neuchâteloise de médecins, séance du 24 octobre 1970, "Dialyse et greffe rénale par le docteur Geromini, Genève", RMSR, 1, 1972, 59.

129 Hofmann 2016, 9-16. 
indirecte dans la documentation des établissements à cette période l'atteste, tout comme l'évolution au niveau international ${ }^{130}$.

\section{Cyclosporine et relève locale}

L'émergence des transplantations hépatiques et cardiaques dans les hôpitaux suisses dépend largement de l'application clinique de la cyclosporine, dont les effets favorables sur la gestion des phénomènes biologiques conduisant au rejet sont unanimement reconnus au début des années 1980. C’est probablement par le biais de conférences de spécialistes, de la littérature scientifique et de discussions informelles que les résultats encourageants obtenus par l'utilisation de la cyclosporine circulent au sein de la communauté scientifique suisse. Dans les établissements au bénéfice d'une expérience provisoirement affirmée dans les années 1970, comme c'est le cas à Genève concernant la transplantation hépatique, le travail expérimental reprend entre médecins. Rohner explique en effet que «le revirement qui se produit ces toutes dernières années nous a poussés à quelques mesures préparatoires, avec l'arrière-pensée de ne pas perdre un temps précieux ${ }^{\mathbf{1 3 1}}$ ». Le cloisonnement des activités expérimentales en marge des activités curatives entrave largement les possibilités d'identifier les acteurs principaux ou de les situer dans un cadre chronologique précis. On sait toutefois que l'envoi de collaborateurs dans des centres de pointe constitue un moyen privilégié pour renforcer l'expertise médicale de la structure administrative au sein de laquelle les activités expérimentales sont menées.

Construite sur les connaissances techniques et scientifiques acquises par un praticien plus âgé, l'expertise est réactualisée pour faire bénéficier, dans le cas genevois, la clinique de chirurgie viscérale, d'une assise scientifique incontestable. À Lausanne, François Mosimann effectue plusieurs séjours d'étude au Queen Elizabeth Hospital de Birmingham dès 1984 pour profiter de l'expérience de spécialistes dans le domaine de la transplantation hépatique tout en

130 Hamilton 2012, 362.

131 HUG, AC, 0037, transplantation, foie, Projet, transplantation hépatique humaine, 10 février 1986, p. 1. 
reconstituant une équipe à Lausanne ${ }^{132}$. L'envoi de «stagiaires » dans un centre spécialisé constitue également un moyen de consolider l'expérience de l'ensemble de l'équipe médicale. Lorsque Rohner transmet son rapport à la direction, Gilles Mentha est en séjour professionnel à l'hôpital Paul-Brousse chez le Pr Bismuth ${ }^{133}$. Enfin, la formation d'une relève locale représente une autre alternative pour s'assurer la disponibilité de médecins qualifiés et faciliter la mise en place d'un programme. À Zurich, Marko Turina remplace Åke Senning à la tête de la chirurgie cardiaque en 1985 après avoir été formé par ce dernier et réactive le programme de transplantation cardiaque ${ }^{134}$.

\subsection{Avancées cliniques}

Au-delà des particularismes locaux qui caractérisent les procédures de gestion, comme la régulation des prises de décision internes, le développement clinique de la transplantation mobilise activement le personnel administratif de chaque hôpital. Contrairement à la transplantation rénale marquée par un monopole tacite des praticiens sur la majorité des étapes entourant sa mise en œuvre, l'établissement des transplantations cardiaques et hépatiques se distingue ainsi par la diversification professionnelle des acteurs impliqués.

\section{Programmes initiaux}

Avec l'utilisation clinique de la cyclosporine, on assiste à une augmentation du nombre de centres de transplantation, notamment aux États-Unis et en Europe. Dans le cas du foie par exemple, plus d'une vingtaine d'hôpitaux au bénéfice d'un programme de transplantations hépatiques y sont dénombrés en $1985^{135}$. Dans les hôpitaux universitaires suisses, le changement de statut scientifique dont béné-

132 Mosimann 1992, 121.

133 HUG, AC, 0037, transplantation, Projet, transplantation hépatique humaine, 10 février 1986, p. 5.

134 www.usz.ch/medien/Documents/sonderbeilagen/110416_50_Jahre_ Herzchirurgie_USZ_Sonderbeilage_NZZ.pdf (consulté en 2019). 
ficie la transplantation, en passant de la procédure expérimentale à un statut de pratique thérapeutique, se traduit par la formulation de revendications écrites. Transmises aux organes administratifs par les médecins directement impliqués, ces demandes sont le plus souvent complétées par la rédaction de rapports destinés aux autorités politiques. Par exemple, Rohner adresse en février $1986^{136}$ une présentation générale des techniques et des coûts probables induits par le développement d'un programme de transplantation hépatique à Genève. Un travail identique est réalisé pour le cœur par Bernard Faidutti en avril $1986^{137}$. Fondées sur une expérience médico-technique déjà établie, ces requêtes successives mettent en évidence une fragmentation des intérêts médicaux par organe. Administrativement, cette atomisation se traduit par un rattachement effectué tacitement selon l'organe et la spécialité chirurgicale à laquelle il est associé: la chirurgie viscérale pour le foie, la chirurgie cardiaque pour le cœur.

Si ces formes d'inclusion renvoient à la diversité des structures administratives qui constituent l'organigramme de l'hôpital, elles soulignent aussi l'éclatement des disciplines "scientifiques" d'où émergent les différents types de transplantations. Au sein de spécialités chirurgicales distinctes les unes des autres, c'est à la faveur d'un confinement marqué que se déroulent les expérimentations. La diversité des procédures médico-techniques qui entourent l'élaboration de types de transplantations dissemblables requiert une faible collaboration entre ces chirurgiens aux domaines d'expertise spécifiques.

Les contacts entre les praticiens se tissent certainement par organe, à «l'extérieur » des établissements par le biais de de rencontres informelles ou de groupes de travail. À ce titre, la création de tels groupes pour la transplantation de foie et cardiaque est réalisée dans le sillage de la création de Swisstransplant, qui en coordonne l'activité ${ }^{138}$.

136 HUG, AC, 0037, transplantation, Projet, transplantation hépatique humaine, 10 février 1986.

137 HUG, AC, 0037, transplantations, cœur, La transplantation cardiaque, état actuel et perspective (sans date).

138 Dès sa création, Swisstransplant pousse les transplanteurs des différents hôpitaux à se rassembler par organe et intègre un représentant de chaque groupe dans le Conseil de fondation (voir chapitre 2). 
Le caractère inédit des pratiques, dépourvues de coutumes régulant leur répartition entre hôpitaux, pousse probablement les chirurgiens à proposer sans concertation un développement clinique rapide avant que la mise en place de programmes tombe sous le coup de directives élaborées par les autorités publiques hors de l'établissement. En effet, cette période d'émergence, soustraite provisoirement à des réglementations sanitaires nationales, est sans doute envisagée comme éphémère face à l'intérêt grandissant de la classe politique pour la gestion de la médecine hautement spécialisée. À cet égard, dès 1989, un groupe de travail est chargé par la Conférence des directeurs cantonaux des affaires sanitaires ${ }^{139}$ (CDS) d'évaluer le nombre de centres dont la Suisse a besoin. En se basant sur une cadence située entre 12 et 24 transplantations par an, ce groupe propose en mai 1989 de limiter à 2 le nombre de centres pour la transplantation de foie, ces propositions n'ayant aucun caractère contraignant ${ }^{140}$.

\section{Éviter un développement incontrôlé}

L'intérêt des instances directives des hôpitaux pour la transplantation se fonde principalement sur la crainte d'assister à un développement entre médecins, sans une prise en compte suffisante des répercussions économiques et organisationnelles sur le fonctionnement habituel de l'institution. Dans un courrier transmis au directeur, le directeur adjoint de l'hôpital cantonal de Genève mentionne "la nécessité de mener des études très complètes avant d'être pris de vitesse par des situations qui pourraient se développer si les greffes de foie, de pancréas et de cœur étaient mises en route à la sauvette ${ }^{141}$ ». À Berne, on redoute que la démultiplication des types de transplantations dans des structures administratives écla-

139 Organe de coordination politique, devenue en 2006 la Conférence suisse des directrices et directeurs cantonaux de la santé, la CDS regroupe l'ensemble des conseillers d'État chargés des affaires liées à la santé de tous les cantons. Elle vise à promouvoir la collaboration intercantonale, celle entre les cantons et la Confédération, ainsi qu'avec les organisations du domaine de la santé.

140 Staatsarchiv Bern, Insel II 757, Verwaltungsauschuss, 15 juin 1989, p. 10.

141 HUG, AC, 0037, transplantation, foie, "note à l'attention de M. Krebs", 24 janvier 1986. 
tées représente une entrave à leur gestion. Le directeur estime, en effet, que «les différents problèmes inhérents à la transplantation ne pourront pas être coordonnés ${ }^{\mathbf{1 4 2}}$ ", ce qui le pousse à proposer de rassembler l'ensemble des types de transplantations dans une même structure administrative. À Lausanne, cette crainte s'exprime sous la forme d'un embargo sur la transplantation d'organes prononcé par le Conseil d'État dès 1985. Il s'agit, comme le mentionne le gouvernement vaudois, d'éviter la mise en place de pratiques sans "en connaître tous les tenants et aboutissants pour les services médicaux et médico-techniques impliqués ${ }^{143}{ }^{1}$. Si la crainte d'un développement incontrôlé est partagée, les tentatives de concertation entre les directions des établissements autour de la mise en œuvre de ces types de transplantations semblent largement marginales. Seul le conseiller d'État genevois Jacques Vernet propose au directeur de son établissement de contacter les quatre responsables des hôpitaux universitaires pour solliciter la CDS dans le but d'entamer une réflexion sur ces pratiques ${ }^{144}$. Les contacts entrepris par le directeur Serge Krebs ne trouvent aucun écho auprès des autres directeurs. Il l'explique par les particularités des situations locales: "soit l'avancement de leurs propres études, soit au contraire l'absence d'une réelle pression à l'heure actuelle du corps médical de leur établissement ${ }^{145}$.»

Ainsi, l'évaluation des conséquences économiques et logistiques de la mise en place de ces pratiques est effectuée localement, à la faveur de procédures internes sous des formes variables selon les établissements. Rapports d'expertise, réunions de groupes de travail pluridisciplinaire, collaborations entre médecins et direction de soins pour organiser la distribution du personnel constituent

142 Staatsarchiv Bern, Insel, Abteilungstransplantation, Transpantationschirugie in Bern, lettre du directeur F. Leu à A. Weber, 4 février 1987.

143 CHUV, boîte "Conseil d'État", Prélèvements d'organes et transplantations au CHUVsituation en mars 1987, p. 3.

144 HUG, AC, 0037, transplantation, foie, "concerne: Projet de transplantation hépatique humaine."

145 HUG, AC, 0037, transplantation, foie, "concerne: Projet de transplantation hépatique humaine", lettre de J. Vernet au président du Collège des chefs de service A. Muller, $1^{\text {er }}$ mars 1986. 
autant de moyens mobilisés pour récolter des données mesurables. Dans cette optique, les instances directives sollicitent également l'expérience acquise par des centres étrangers. À Genève, un collaborateur de la Commission de direction passe deux jours à Paris dans les hôpitaux Paul-Brousse et de la Pitié-Salpêtrière pour prendre connaissance des prescriptions organisationnelles développées dans les services chargés de la réalisation des transplantations ${ }^{146}$.

Si l'engagement de personnel soignant représente une charge financière sur le long terme que les établissements refusent de supporter, certaines dépenses concernant l'adaptation des infrastructures sont inévitables. C'est le cas de l'agrandissement des laboratoires d'analyse des établissements dans lesquels les nombreux tests visant à établir la compatibilité entre le donneur et le receveur sont effectués. Si un laboratoire de référence pour l'histocompatibilité existe à Genève, son activité est surtout liée à la centralisation des résultats médicaux obtenus et la création des procédures communes d'examens biologiques pour l'ensemble des hôpitaux. Avec la perspective d'une diversification des types de transplantations, la certitude d'une augmentation inévitable du nombre d'examens à mener sur place dans un délai bref est unanimement partagée. Toutefois, les transformations des infrastructures sont largement façonnées par les impératifs budgétaires qui imposent une mise à disposition de moyens financiers limités. À Berne, alors que les premières transplantations hépatiques sont imminentes, le conseil d'administration accepte de débloquer des fonds, mais réduit considérablement le projet présenté par le corps médical ${ }^{147}$.

Dans ce contexte de contrôle strict des dépenses, la promotion de la mise en place de programmes par les transplanteurs vise à associer la transplantation à une pratique thérapeutique habituelle pour un hôpital de pointe. À cet égard, Rohner présente la transplantation dans un rapport transmis au Conseil comme étant «en passe de prendre place dans les possibilités thérapeutiques qu'un

146 HUG, AC, 0037, transplantations, foie, "note à l'attention de M.A. Rochat, directeur général adjoint ", 3 juillet 1986.

147 Staatsarchiv Bern, Insel II 952, Verwaltungsausschuss, Protokolle und Akten 1987/13, 19 février 1987. 
pays développé se doit d'offrir à sa population ${ }^{148}$ ". Dépourvu du caractère exceptionnel de ses débuts, son développement dans les établissements universitaires est légitime au même titre qu'une intervention chirurgicale lourde. La cohérence de l'offre thérapeutique, envisagée à l'échelle de l'établissement, est également présentée comme un élément prépondérant. À propos de la transplantation cardiaque, Faidutti explique qu'il comprendrait mal "pourquoi seraient pratiquées des transplantations rénales, des greffes de moelle osseuse, voir [sic] des transplantations hépatiques alors que les transplantations cardiaques seraient récusées ${ }^{149}{ }^{\text {». }}$

\section{Planifier et évaluer}

Si le renoncement à la constitution de programmes laisse craindre une diminution du dynamisme scientifique qui caractérise un hôpital de pointe, les instances directives et différents conseils y voient une menace potentielle pour l'ensemble de la structure sanitaire cantonale. Dans une vision où l'hôpital universitaire est investi d'une mission centralisatrice dans la prise en charge des habitants d'une région, souvent assimilée au canton, il se doit d'offrir les soins les plus poussés. Renoncer à certaines prestations médicales équivaut sinon à une hausse de coûts pour les assurances et le canton, en tout cas à des tracasseries administratives. À ce titre, le conseil d'administration de l'Inselspital évoque les arrangements qui devraient être trouvés avec les autres centres suisses ${ }^{150}$.

Ce processus prend la forme d'une planification interne, dans laquelle les enjeux économiques sont certes mesurés, mais sans représenter des critères exclusifs. Ce travail préparatoire, mené sur une période d'environ deux ou trois ans selon les établissements, conduit à la réalisation de premières transplantations, le plus souvent autorisées à titre provisoire. Ce procédé présente une sécurité sup-

148 HUG AC, 0037, transplantations, cœur, lettre du président du Collège des chefs de service A. Müller au président de la Commission administrative J. Vernet, 10 avril 1986.

149 HUG, AC, 0037, transplantations, cœur, La transplantation cardiaque, état actuel et perspective (sans date), p. 13.

150 Staatsarchiv Bern, Insel II 957, Verwaltungsausschuss, Protokolle und Akten 1987/23, 15 juin 1989, p. 25. 
plémentaire pour l'établissement en offrant la possibilité de prendre connaissance des répercussions logistiques et financières ou des imprévus techniques. Ainsi le planificateur de l'hôpital cantonal de Genève explique à la direction qu'il «est difficile de saisir tous les éléments qui permettent d'annoncer le coût réel de ce type d'intervention $^{151} »$. Il propose donc d'autoriser six transplantations d'essai pour chaque organe "étant entendu que ces programmes permettront de relever de manière exemplaire tous les phénomènes techniques, financiers, médicaux, etc., permettant ensuite de faire un rapport et de conclure sur l'introduction définitive de ces méthodes ${ }^{152}$ ». La qualité des résultats obtenus et la valeur thérapeutique ne représentent qu'un aspect inclus dans un ensemble de motifs hétéroclites plus large sur lequel la mise en place de programme est évaluée. À ce titre, le président de la Commission administrative insiste sur le moyen d'obtenir des connaissances précises «sur les conditions économiques dans lesquelles se dérouleraient ces opérations, pendant cette période expérimentale ${ }^{153}[\ldots]$ ». Après être parvenu à répartir le personnel disponible par rocade, l'embargo sur la transplantation hépatique est levé à Lausanne en août 1988. Comme à Genève, une réévaluation de l'impact est prévue sous la forme d'un bilan «concernant les retentissements de l'introduction de cette nouvelle prestation ${ }^{154}$ ».

Dans cette optique, la "première» constitue une étape du processus d'organisation, plus que son aboutissement. Elle s'apparente à une répétition générale pour laquelle des précautions particulières sont prises pour assurer les meilleures conditions logistiques. Dans la crainte de manquer de personnel, les urgences de Morges et d'Yverdon sont sollicitées par Mosimann pour décharger celles du Centre hospitalier universitaire vaudois (CHUV) le moment venu ${ }^{\mathbf{1 5 5}}$.

151 HUG, AC, 0037, transplantations foie, rapport de M. Maricot au bureau de direction, 13 janvier 1987.

152 Idem.

153 HUG, AC, 0037, transplantations, foie, lettre du président de la Commission administrative J. Vernet au directeur général de l'hôpital, 22 juillet 1987.

154 CHUV, boîte "Conseil d'État", "Proposition à soumettre au Conseil d'Etat", 20 mai 1988 [adopté par le Conseil d'Etat le 27 mai 1988].

155 CHUV, procès-verbal n 47/1988 de la séance de la direction du CHUV, 19 janvier 1988, p. 3. 
Les spécificités médico-techniques liées à chaque type de transplantation et les conséquences de leur conversion en phénomènes économiquement mesurables influencent sans doute la durée de cette période prospective. C'est la possibilité de mettre en place «sans l'engagement de collaborateur soignant additionnel ${ }^{156}$ » qui conduit la direction générale du CHUV à proposer au Conseil d'État l'autorisation de pratiquer des transplantations cardiaques pour le début de l'année 1987. Comme l'explique Faidutti à Genève dans son rapport, pour assurer la mise en place de programme de transplantation cardiaque, "aucun besoin particulier ni en locaux ni en personnel n'est nécessaire dans l'état actuel ${ }^{157}$ ». La transplantation hépatique présente des difficultés techniques spécifiques qui allongent la durée de l'opération et mobilisent un effectif de soignants conséquent. À Lausanne, l'embargo sur la transplantation de foie est ainsi maintenu afin de "poursuivre les études en vue de couvrir les besoins en force de travail supplémentaire ${ }^{158}$ ». Sans recrutement d'employés additionnels, un protocole exact fixant le déroulement de cette prestation est notamment requis par la direction des soins. Il vise à établir avec précision chaque étape de l'intervention pour prévoir la répartition des employés entre transplantation et activité habituelle ${ }^{159}$.

Si ces types de transplantations sont normalisés progressivement sous forme de programmes, leur adaptation aux contraintes économiques se poursuit. Comme les autres pratiques médicales parties prenantes de l'offre thérapeutique des hôpitaux, leur gestion est influencée par les impératifs budgétaires. Au CHUV par exemple, l'adaptation des procédures médicales postopératoires permet en 1991 de minimiser le besoin en personnel soignant grâce à une

156 CHUV, boîte "Conseil d'État", Extraits du procès-verbal du Conseil d'État, 25 mars 1987.

157 HUG, AC, 0037, transplantations, foie, Projet, transplantation hépatique humaine, 10 février 1986, p. 2.

158 CHUV, boîte "Conseil d'État", Prélèvements d'organes et transplantations au CHUV - situation en mars 1987, p. 3.

159 CHUV, procès-verbal n 47/1988 de la séance de la direction du CHUV, 19 janvier 1988 , p. 2. 
réduction de la durée de l'isolement dans le cas de la transplantation cardiaque $^{160}$.

\subsection{Conclusion}

Loin d'être le fruit d'une politique hospitalière planifiée, la transplantation, qu'il s'agisse du rein, du foie ou du cœur, se développe avant tout sous l'impulsion de quelques médecins hospitaliers. Le rôle prépondérant des initiatives individuelles constitue en effet une caractéristique commune au développement de tous les types de transplantations dans chaque hôpital.

Dans le cas de la transplantation rénale, les rapports interprofessionnels qui règlent tacitement les relations que les médecins entretiennent avec les instances directives leur confèrent une marge de manœuvre importante. Si le personnel administratif se charge d'assurer la bonne marche financière de l'établissement, il laisse au corps médical le soin d'évaluer l'opportunité du développement de pratiques. Selon un processus informel et dans la mesure des ressources économiques disponibles, les médecins prennent en main entre experts, en marge, l'organisation de la transplantation rénale. Si le processus de mise en œuvre qui en découle est difficile à retracer de manière détaillée, elle illustre le fonctionnement des hôpitaux à cette période.

Dans le cas de la mise en place des transplantations hépatiques et cardiaques, on constate qu'en dépit des impératifs budgétaires omniprésents qui influencent l'offre thérapeutique, aucun hôpital n'oppose un refus catégorique au développement de la transplantation. La légitimité du corps médical à définir les pratiques prioritaires, même dans des contextes de gestion contrôlée dont le personnel administratif est garant, reste un élément essentiel sur lequel se fonde le fonctionnement des hôpitaux. Le prestige et la mission sanitaire d'un hôpital universitaire constituent le point de contact autour duquel les intérêts professionnels variés se rejoignent.

160 CHUV, procès-verbal $n^{\circ} 2 / 1993$ de la séance de la direction du CHUV, 21 novembre 1993, p. 2. 
En l'absence de plan directeur relatif au développement des différents types de transplantations dans les années 1960 comme dans les années 1980, c'est le savoir-faire "local» cadré par les exigences économiques de bonne marche des hôpitaux qui marque durablement la configuration de l'offre sanitaire dans ce domaine. Alors que la coopération entre hôpitaux dans la phase initiale est très marginale, les groupes de travail formés par les transplanteurs sont les initiateurs et moteurs de l'organisation nationale de la transplantation. C'est ce que nous examinerons dans le chapitre suivant en nous centrant sur le rôle clé joué par Swisstransplant. 


\title{
Chapitre 2
}

\section{La création et le fonctionnement de Swisstransplant}

\author{
Alexia Cochand
}

La création de groupes de travail pour la transplantation rénale et de moelle osseuse à la fin des années 1960 avait jeté les bases d'une gestion coordonnée des transplantations. Dans cette période la mise en place de programmes de transplantation est réalisée par organe sous l'impulsion de médecins actifs dans des centres indépendants les uns des autres. Dès 1985, le domaine de la transplantation connaît un tournant majeur avec la création de Swisstransplant qui en deviendra un acteur de premier plan.

En retraçant le développement structurel de Swisstransplant, ce chapitre vise à mettre en évidence la transition d'une organisation informelle et décentralisée de la transplantation vers une structure unique à vocation nationale. Loin d'être évidente à sa création, la reconnaissance de Swisstransplant est progressive et n'est acquise définitivement qu'au début des années 2000 dans le cadre de la préparation de la loi fédérale sur la transplantation. La compétence dont dispose la fondation dans la gestion médicale et organisationnelle est en effet façonnée par l'extension au fil du temps de ses structures internes (principalement le Conseil de fondation, le Comité exécutif et la Coordination) qui témoigne de son influence 
croissante. S'appuyant principalement sur les procès-verbaux des structures constitutives de la fondation, ce chapitre examinera plus particulièrement l'évolution des structures déployées et les modifications apportées à l'organigramme jusqu'à la fin des années 1990. Il s'agit d'identifier les circonstances de leur création et les objectifs qu'elles ont cherché à atteindre. Nous retracerons également les activités menées par les différentes structures dirigeantes en nous arrêtant, le cas échéant, sur les divergences entre les différents membres de la fondation. Particulièrement propices pour mettre en évidence les aléas d'un processus de développement élaboré par la discussion dans les conseils de gestion, ces oppositions permettent d'identifier les priorités poursuivies par la fondation.

\subsection{Mise en place des structures et définition des activités (1985-1991)}

\section{Création de la fondation}

L'acte constitutif de la fondation est signé le 4 mars 1985 à Genève par sept membres aux intérêts professionnels variables afin de "favoriser, développer et coordonner en Suisse la transplantation d'organes, de tissus et de cellules ${ }^{161}{ }^{\prime}$. Trois d'entre eux - les médecins Felix Largiadèr, chirurgien à l'hôpital de Zurich, Michel Jeannet de l'unité d'immunologie des transplantations de l'hôpital de Genève, et Bruno Speck de la clinique d'hématologie de Bâle - sont déjà au bénéfice d'une large expérience professionnelle à l'étranger et en Suisse.

$\mathrm{Au}$ sein du département de chirurgie de l'université de Minneapolis, Largiadèr travaille notamment à la transplantation expérimentale et clinique. Dès son retour en Suisse en 1966, il participe activement au développement thérapeutique de la transplantation rénale et pancréatique au sein de la clinique de chirurgie de l'hôpital de Zurich. Médecin-chef depuis 1976 à la clinique de

161 Acte constitutif de Swisstransplant fondation suisse pour la transplantation, acte de Maître Paul Tournier du 4 mars 1985, p. 1. 
chirurgie, il assume officiellement la fonction de directeur du nouveau département de chirurgie viscérale à Zurich le 16 avril $1985^{162}$, un mois après la création de Swisstransplant. Quant à Jeannet, après un séjour à Boston en 1968 où il travaille sous la direction de Paul Snowden Russell, actif dans l'élaboration de test de typage dès le début des années 1960, il s'attelle à Genève à la standardisation des techniques et des tests de groupage leucocytaire pour l'ensemble du pays. Ce travail effectué en collaboration avec les laboratoires de chaque hôpital universitaire est coordonné et contrôlé par le laboratoire de l'hôpital cantonal de Genève de l'unité d'histocompatibilité qu'il dirige dès $1972^{163}$. Le laboratoire est reconnu en 1982 par la CDS comme laboratoire national de référence pour l'histocompatibilité ${ }^{164}$. Enfin, Speck est actif dans le domaine de la transplantation de moelle, au bénéfice d'une étroite collaboration à Leiden avec Jon van Rood, immunologiste néerlandais, Prix Nobel de médecine en 1978 avec Jean Dausset et George Snell pour son travail dans la mise au jour du système HLA ${ }^{165}$ et fondateur d'Eurotransplant en 1967. De retour en Suisse, Speck met en place l'un des premiers programmes mondiaux de transplantation de moelle à Bâle en $1973^{166}$ et y dirige la clinique d'hématologie à partir de 1979. Conjointement aux activités professionnelles qu'ils conduisent dans leur établissement respectif, ces trois médecins siègent dans un des groupes de travail formés à la fin des années 1960, lors de la mise en place de la majeure partie des programmes de transplantation rénale dans les hôpitaux suisses. Au vu de leurs compétences, il est fort probable

162 www.vis.usz.ch/ueber-die-klinik/geschichte/Seiten/felix-largiader.aspx (consulté en 2019).

163 HUG, Archives centrales (AC), CA_HC, 20.01.1973, p. 11.

164 Correspondance, "Laboratoire national de référence pour l'histocompatibilité en matière de transplantations rénales et de moelle osseuse", 27 septembre 1989, p. 1.

165 Antigènes des leucocytes humains (abrégés HLA). Les scientifiques, qui s'intéressent à ces antigènes situés à la surface des leucocytes depuis les années 1950, les identifient comme les plus importants marqueurs de l'identité chez l'homme et considèrent qu'ils jouent un rôle central dans le processus de rejet des organes transplantés. Sur cette base, ils proposent un système de typage cellulaire spécifique pour permettre l'appariement du donneur et du receveur.

166 Buckner 2000. 
que Largiadèr joue un rôle central au sein du groupe de travail pour la chirurgie de transplantation, tout comme Jeannet et Speck dans celui qui rassemble les experts du domaine de l'histocompatibilité.

Aux côtés de ce noyau d'acteurs dont l'avenir professionnel dans le domaine de la transplantation dépend de son expansion, l'acte de fondation met en évidence la participation de l'Académie suisse des sciences médicales (ASSM). Acteur institutionnel incontournable par son implication dans la définition des critères de la mort cérébrale en Suisse ${ }^{167}$, l'ASSM est représentée par Bernard Courvoisier, président de la commission centrale d'éthique médicale. Le groupe fondateur de Swisstransplant compte également un représentant de l'entreprise Sandoz-Pharma qui a identifié les effets de la cyclosporine dans la gestion du rejet et commercialisé la molécule au début des années 1980. Par le biais de la participation de Rudolf Schmidt, chercheur au sein du département de recherche clinique à Bâle ${ }^{168}$, la fondation s'assure de la disponibilité d'un intermédiaire direct auprès d'une source de financement potentiel. Afin de donner une visibilité à la fondation au-delà de la sphère médicale, la participation de deux hommes politiques est sollicitée, sans doute à l'instigation des médecins. Dans cette optique, le conseiller d'État genevois Jacques Vernet, du département de la prévoyance sociale et santé publique ${ }^{169}$, et le conseiller aux États saint-gallois Jacob Schönenberg, membre notamment du conseil de la Banque nationale ${ }^{170}$, rejoignent le groupe des membres fondateurs et intègrent le Conseil de fondation.

\section{Recherche de financements et de visibilité}

Selon ses statuts, la fondation Swisstransplant est administrée par un conseil d'au moins quinze membres, dont la composition fait l'objet de directives précises. Au groupe des membres fondateurs

167 Bellanger et Steinbrecher 2002.

168 Ce que laisse penser la publication d'articles à la même période à l'instar de celui de Schmidt du département de recherche clinique de l'entreprise Sandoz à Bâle: Schmidt Rudolf, "Dose-finding studies in clinical drug development", European Journal of Clinical Pharmacology, 34(1), 1988, 15-19.

169 https://hls-dhs-dss.ch/fr/articles/006840/2011-08-31/(consulté en 2019).

170 https://hls-dhs-dss.ch/fr/articles/033657/2010-11-30/ (consulté en 2019). 
sont adjoints des représentants des groupes de travail spécialisés qui seront constitués, des centres de transplantation, des milieux bancaires, de l'industrie et de la Caisse de réassurance pour longues maladies.

Unique espace de décision de Swisstransplant, le Conseil de fondation se réunit une fois par année. Ces réunions sont l'occasion pour les membres d'élaborer le programme d'activités de la fondation. Loin de s'apparenter à l'établissement d'un plan de développement précis, il se construit au fil des réunions par les propositions émises par les membres. Les discussions dont nous avons connaissance par l'entremise des procès-verbaux se concentrent sur deux thématiques jusqu'à la fin des années 1980: d'une part les aspects organisationnels et médicaux de la transplantation, d'autre part l'information du public au sujet du don d'organes.

Concernant les aspects organisationnels et médicaux, il s'agit le plus souvent de propositions ou réflexions centrées sur un aspect spécifiquement lié au domaine d'activité des médecins concernés. Bruno Speck aborde dès la première réunion la nécessité de mettre sur pied un registre de donneurs non apparentés pour la moelle osseuse ${ }^{171}$. Ce type de propositions discutées brièvement, sans déboucher sur des décisions concrètes, illustrent la fragmentation de l'organisation de la transplantation au début des années 1980. Toutefois, face aux intérêts médicaux propres à chaque type de transplantation, ces réunions permettent l'élaboration de réflexions communes concernant des thématiques organisationnelles. La décision prise lors de la réunion annuelle du mois de juin 1986 d'établir une statistique de tous les organes greffés en est un exemple ${ }^{172}$.

Dès les premières séances, l'information du public est perçue par l'ensemble des membres, quoique avec une intensité variable, comme une des tâches principales de la fondation. Les procès-verbaux laissent cependant apparaître certaines dissensions entre l'intérêt des médecins à développer des projets scientifiques et la mise en place de stratégies visant à informer la population. Induit par l'absence d'un

171 Procès-verbal du Conseil de fondation (P-V CF), 4 décembre 1985, p. 2.

172 P-V CF, 4 juin 1986, p. 4. 
programme d'activité préalablement défini et amplifié par l'unicité de la structure dirigeante qu'aucun organe subordonné ne complète, ce mélange d'intérêts est toutefois maîtrisé par quelques membres "emblématiques ». Transplanteurs ou acteurs directement impliqués dans le processus médical de la transplantation, ils sont souvent également membres fondateurs, ce qui leur confère une autorité sur les autres participants. À cet égard, Largiadèr dirige les débats en définissant les objectifs de la fondation. À propos d'une proposition de soutien à apporter à une recherche, il estime que "ces différents projets scientifiques ne touchent pas directement à [sic] la fondation et qu'un projet d'intérêt plus général devrait être formulé ${ }^{173}$ ». Cette remarque clôt définitivement la discussion sur le sujet.

Si la définition d'objectifs communs concernant l'organisation de la transplantation ou l'information du public ne trouve pas d'unanimité au sein du conseil, la réalité matérielle de la fondation conduit les membres à s'accorder sur le besoin d'une recherche active de financement. Inhérente au statut juridique de la fondation, l'absence de capital impose d'elle-même des limites aux activités scientifiques ou à visée informative. L'impossibilité, par exemple, de disposer d'un secrétariat qui centralise l'information représente un obstacle pour l'information du public ${ }^{174}$. Dès lors, une large partie de l'activité concrète effectuée par les membres s'articule autour de la recherche de sponsors. La principale stratégie développée est la sollicitation d'institutions ou d'acteurs inscrits dans le secteur privé, comme des banques ou assureurs ${ }^{175}$ ou des pouvoirs publics cantonaux ${ }^{176}$. Financement pour soutenir une action spécifique et ponctuelle ou pour assumer des frais constants et durables comme la production de cartes de donneurs, ces demandes sont réglées au cas par cas.

La recherche de fonds oblige la fondation à préciser ses ambitions concrètes. Elle implique que ses membres définissent des objectifs clairs à présenter aux éventuels donateurs. Ainsi, dans un contexte

173 P-V CF, 4 décembre 1985, p. 1.

174 Idem, p. 3.

175 Idem, p. 4.

176 Idem. 
de fonctionnement où la situation matérielle est limitée, un consensus se forme autour de la nécessité d'axer les efforts sur la diffusion d'information sur la transplantation et la fondation dans la sphère publique. Lors d'une séance, l'un des membres indique "qu'il est important que le nom de Swisstransplant soit davantage connu du public, comme celui de la REGA ou de la Fondation pour les paraplégiques $^{177}$ ». L'ambition est de donner suffisamment de notoriété à la fondation pour faciliter la sollicitation de futurs «sponsors $^{178}$ ». Les activités de recherche et autres projets sont relégués provisoirement au second plan, en attendant que sa visibilité soit considérée comme suffisante. Cette priorité tient en partie aux alliances nouées avec d'autres acteurs. En 1986, une année après la création de la fondation, Largiadèr relate avoir été approché par la branche zurichoise du Lions Club qui propose de mettre à disposition des moyens financiers et matériels pour soutenir les actions menées par Swisstransplant. Jouissant d'un réseau diffus d'acteurs mobilisables dans différents secteurs d'activité, le Lions Club offre également de se charger de la collecte de fonds auprès de sponsors. Dans un esprit de cohérence avec les objectifs philanthropiques qui structurent cette institution, les fonds mis à disposition ne doivent financer que des projets liés à l'information du public ${ }^{\mathbf{1 7 9}}$ et l'aide apportée à la fondation ne peut être que temporaire, sans toutefois que la durée ne soit définie. Swisstransplant saisit cette opportunité de collaboration qui lui permettra en outre de tisser des liens hors de la sphère médicale. L'orientation des activités menées par la fondation en est donc considérablement marquée sans faire toutefois l'unanimité. Le principal objet de dissension soulevé par le partenariat avec le Lions Club concerne la compétence des membres de cette organisation à s'exprimer en public sur des questions liées à la transplantation. Certains médecins de la fondation craignent que la transplantation fasse l'objet d'informations trop simplifiées ou erronées par des personnes non spécialisées. Pour permettre à la

177 P-V CF, 10 mars 1987, p. 4.

178 Idem.

179 P-V CF, 4 juin 1986, p. 2. 
fondation de garder le contrôle sur les activités menées par le Lions Club et résoudre ces tensions, le conseil décide de créer le groupe de travail Swisstransplant-Lions Club constitué de membres des deux institutions ${ }^{180}$. Sa présidence est confiée à Jean-Claude Scherz, membre du Lions Club, qui sera intégré au Conseil de fondation pour jouer le rôle d'intermédiaire entre Swisstransplant et le groupe de travail.

\section{Informer le public}

Entre 1988 et 1991, les activités sont centrées sur la récolte de fonds grâce à des "sponsors" ou à l'organisation d'événements publics, dont les bénéfices sont reversés à la fondation. La recherche est diffuse, s'orientant aussi bien sur des soutiens obtenus dans le cadre de manifestations très diverses (une démonstration de dressage de chevaux de l'École espagnole de Vienne ou un bal de bienfaisance à Zurich) que la sollicitation d'entreprises pour un don ponctuel ${ }^{181}$. Ces activités permettent de récolter de l'argent, mais également de donner une dimension publique à la transplantation et au nom de Swisstransplant.

La première action issue de la collaboration avec le Lions Club réside dans le lancement d'une carte de donneur d'organes. Présentée officiellement lors d'une conférence de presse en 1987, elle ne fait pourtant pas l'unanimité parmi les transplanteurs, comme l'illustrent les propos de Gilbert Thiel:

«[...] Il y a plusieurs années, on avait abouti à la conclusion que cette carte n'était pas indispensable et que son lancement pouvait même avoir des effets négatifs : par exemple, le fait qu'un donneur potentiel ne possède pas la carte pouvait être interprété par sa famille comme un refus de donner ses organes; d'autre part, l'expérience des pays voisins a montré que la diffusion de telles cartes reste en général limitée à une petite fraction de la population, quels que soient les efforts entrepris pour sa promotion ${ }^{182}$.»

180 Idem, p. 2.

181 P-V CF, 22 juin 1988, p. 4.

182 P-V CF, 10 mars 1987, p. 2. 
En dépit de certains avis mitigés, la majorité des membres du conseil souhaite profiter du soutien offert par le Lions Club. La carte représente un moyen privilégié de développer une présence dans l'espace public. Le recentrement ultérieur des activités sur les problématiques organisationnelles ou médicales reste une motivation sous-jacente. Plus que toute autre action, la carte de donneur doit d'abord contribuer à faire connaître la fondation auprès de la population. «Après le lancement de cette dernière [...] la Fondation sera connue et pourra s'adresser à d'autres sponsors. De ce fait le "Sponsoring-Konzept" profite directement à la fondation ${ }^{183}$ ", explique un des membres pour justifier son soutien à ce projet.

Parallèlement, le groupe de travail élabore du matériel pour soutenir les activités de promotion du don d'organes et d'information sur la fondation. Dans l'optique de la tenue de conférences publiques, des diapositives et des brochures sont spécialement conçues, de même qu'une documentation informative à joindre à chaque carte de donneur ${ }^{184}$. Avalisés par la fondation, ces supports représentent un moyen de communication uniformisé et participent à la construction d'un discours officiel sur la transplantation. Les données certifiées sur la transplantation que ces documents contiennent contribuent à lier l'image de Swisstransplant à la gestion médicale et organisationnelle de la transplantation.

La diffusion d'information passe également par une présence accrue dans la presse ${ }^{185}$. Des encarts sont mis à disposition gratuitement par des quotidiens, dont le Tages-Anzeiger, ou des agences de presse contactées par le Lions Club ${ }^{186}$, des articles sur le thème «donner, c'est la vie» sont publiés. Ce procédé proactif de diffusion de l'information assure une apparition régulière de la thématique de la transplantation dans la presse ${ }^{187}$. Des articles et tirés à part sont également publiés dans des revues médicales, comme le Bulletin des

183 Idem, p. 4.

184 P-V CF, 22 juin 1988, p. 4.

185 P-V CF, 7 décembre 1988, p. 2.

186 Rapport d'activité de Swisstransplant pour 1991, 26 mai 1992, p. 1.

187 P-V CF, 7 décembre 1988, p. 2. 
médecins suisses. La mobilisation d'acteurs médicaux joue un rôle important dans le processus de diffusion d'information développé par la fondation. Ce dernier compte largement sur la solidarité du corps médical pour jouer le rôle de relais dans l'information sur le don d'organes. Il s'agit donc de s'assurer que les médecins eux-mêmes soient suffisamment informés sur la transplantation et conscients du rôle qui est le leur.

Des lettres d'information et de sensibilisation à la transplantation et au don d'organes sont envoyées à l'ensemble des médecins appartenant au Lions Club en Suisse ${ }^{188}$. Des contacts sont noués par le groupe de travail avec des organisations telles que la Société suisse des paraplégiques ou des pharmaciens et des droguistes pour leur proposer de distribuer des brochures à leurs membres ou d'en mettre à disposition dans leurs locaux ${ }^{189}$. Ces documents concernent le plus souvent la transplantation dans sa globalité, mais sont parfois spécifiques à un organe en particulier. Les centres de don de sang sont également sollicités pour mettre à disposition des brochures sur la transplantation de moelle ${ }^{190}$.

La collaboration avec le Lions Club joue également un rôle capital dans la mise en place et le financement des infrastructures de Swisstransplant. En effet, l'institution philanthropique finance la création de centrales d'information au Tessin et à Schaffhouse ${ }^{191}$. Représentantes directes de la fondation dans la sphère publique, ces structures sont chargées de la diffusion de brochures ou de carte de donneurs à toute personne ou organisme intéressé. Parallèlement à la distribution de cartes lors d'événements, la fondation peut désormais, par l'entremise de ces centrales, répondre aux demandes concrètes de la population.

Au moment où l'organisation philanthropique zurichoise décide, conformément à son mode de fonctionnement, de mettre un terme au partenariat développé avec la fondation pour se consacrer à d'autres

188 P-V CF, 10 mars 1987, p. 4.

189 P-V CF, 13 juin 1989, p. 1; P-V CF, 5 décembre 1989, p. 3-4.

190 P-V CF, 13 juin 1989, p. 4.

191 P-V CF, 4 juin 1986, p. 3. 
causes, les bases d'un système d'information et de promotion du don sont jetées. Le lancement de la carte de donneur devient le principal outil de recrutement des donneurs et de diffusion de brochures explicatives sur la transplantation et le don d'organes. La large mobilisation de sponsors permet à la fondation d'acquérir une visibilité dans des milieux extramédicaux que la fondation aurait eu plus de difficulté à atteindre sans le partenariat établi avec le Lions Club.

\section{Les groupes de travail par organe}

Si la recherche de soutien financier occupe une place importante dans les activités du Conseil de fondation, le partage d'informations sur les aspects médicaux et logistiques de la transplantation n'est pas pour autant négligé. Dès 1987, les réunions annuelles donnent lieu à la présentation des rapports d'activité des groupes de travail de transplantation, composés de chirurgiens et de médecins spécialistes issus des différents centres et rassemblés par organe.

Le processus de rapprochement entre les médecins est le résultat d'initiatives personnelles, parfois antérieures à la création de Swisstransplant. Les groupes de travail pour la transplantation rénale et de moelle ont une activité scientifique déjà bien marquée au milieu des années 1980. Loin d'être de simples réunions d'experts, ils sont l'occasion de mettre en commun l'expérience de la pratique clinique de la transplantation. L'élaboration d'une politique de développement et de gestion des problèmes logistiques et organisationnels (échanges entre les centres, transport des organes...) est au centre des préoccupations. Le groupe pour la transplantation de moelle établit, par exemple, une collaboration avec la Croix-Rouge à Berne. L'identification et l'enregistrement des donneurs y sont effectués et centralisés pour permettre à la transplantation à partir de donneurs non apparentés de gagner en importance ${ }^{192}$.

Dans les rapports qu'ils entretiennent avec la fondation, ces deux groupes bénéficient d'une certaine indépendance due à leur ancienneté. C'est avec la diversification des types de transplantations que les autres groupes sont créés à la fin des années 1980. 
Le rôle de Swisstransplant dans le processus de regroupement des transplanteurs de différents centres demeure assez flou. Dans le cas de la création du groupe de travail pour la transplantation hépatique, le regroupement semble s'être effectué sans le concours de la fondation. Dans un rapport présenté devant le Conseil de fondation, François Mosimann, chirurgien au CHUV, explique:

«En février 1987, les services de chirurgie universitaires suisses, ainsi que les responsables des soins intensifs de plusieurs hôpitaux se sont réunis pour discuter de l'organisation des transplantations hépatiques. Il a été décidé de greffer des foies à Zurich, Lausanne, Berne et Genève ${ }^{193}$.»

Un groupe est parfois créé grâce à l'impulsion d'un membre de la fondation. À cet égard, Largiadèr décide de prendre contact et "de discuter avec les chirurgiens spécialisés dans la transplantation cardiaque de la mise sur pied d'un groupe de travail séparé ${ }^{194}$ ».

Si ces espaces de travail jouent un rôle important dans la prise en charge des aspects médicaux et organisationnels de la transplantation, ils représentent structurellement un dépassement du cloisonnement imposé par la pratique hospitalière. Au sein de leurs établissements respectifs, les chirurgiens transplanteurs sont répartis par spécialité médicale sans contact direct avec des pairs. Les échanges d'expériences entre experts dans des domaines de compétence peu répandus comme la transplantation contribuent à une collaboration interhospitalière. Ce processus de mise en commun des compétences jette les bases du processus de collaboration et de coordination de la transplantation entre les institutions. Ces groupes sont représentés au sein de la fondation par l'intégration d'un de leurs membres au Conseil de fondation. Dans le cas du rein et de la moelle, des membres des groupes de travail siègent depuis la création de Swisstransplant. Conformément aux statuts de l'acte de fondation, il est décidé d'intégrer automatiquement un représentant de chaque nouveau groupe ${ }^{195}$.

193 P-V CF, 2 décembre 1987, p. 4.

194 P-V CF, 4 juin 1986, p. 3.

195 P-V CF, 10 mars 1987, p. 5. 
Pour Swisstransplant, la participation d'un des membres dans le Conseil de fondation constitue un moyen d'établir un lien direct entre la réalité médicale et logistique de la pratique et la fondation. Le Conseil de fondation est ainsi directement informé de l'état des lieux de la transplantation en Suisse. S'ils sont partie prenante de la fondation, ces groupes gardent toutefois une large indépendance dans la définition de leur mode de fonctionnement. Les règles d'admission sont établies selon des critères propres aussi bien que par la nature des liens que les transplanteurs tissent entre eux.

\section{Le comité exécutif et l'organisation des activités de transplantation}

Si l'information du public est assurée entre 1988 et 1991, le financement d'activités distinctes est un problème récurrent qui limite l'ampleur des actions entreprises par Swisstransplant. Le Conseil de fondation procède à des recherches de fonds permanentes pour assurer la réalisation d'autres types d'activités. Le manque de moyens limite la réalisation d'actions, comme l'organisation de colloques scientifiques, et affecte directement le développement structurel de la fondation. La création de postes est en effet financée à brève ou moyenne échéance, à l'instar d'un poste de secrétaire que le Crédit Suisse propose de financer à $10 \%$ ou $20 \%$ pour trois ans $^{196}$. Swisstransplant compte également sur des arrangements avec des institutions médicales mieux loties financièrement. Elle bénéficie par exemple largement du soutien de l'hôpital cantonal de Genève au sein duquel se trouve le laboratoire d'histocompatibilité. Le secrétariat de cette institution se charge de centraliser les données sur la transplantation, tout en endossant le rôle de centrale d'information du public pour la Suisse romande ${ }^{197}$. Ces accommodements illustrent le statut institutionnel fragile de la fondation dont le fonctionnement n'est assuré qu'à court terme.

196 Idem, p. 5.

197 Correspondance, "Poste de secrétaire pour l'organisation Swisstransplant à Genève", 29 mars 1990, p. 1-2. 
La lenteur du processus décisionnel induite par le nombre élevé de membres siégeant au Conseil de fondation représente également une restriction des possibilités de développement général de la fondation. À partir de mars $1987^{198}$, les membres décident de se réunir deux fois par année. Toutefois, l'intensification du nombre de rencontres n'entraîne pas une hausse significative des décisions concrètes. Le Conseil de fondation joue plutôt le rôle d'espace de discussion où les membres relatent leurs activités en lien avec la transplantation. La fin du partenariat avec le Lions Club, annoncée en 1990, laisse également planer le doute sur les conditions dans lesquelles la poursuite des activités d'information du public pourra être menée. Sans acteurs entièrement dévolus à la recherche de fonds, le financement nécessaire pour mener ce type d'activité est menacé ${ }^{199}$.

Dans ce contexte matériel et structurel limitant les capacités du Conseil de fondation à mettre en place un plan de développement, les membres décident, sur proposition de Largiadèr lors de la réunion annuelle de 1990, de créer un Comité exécutif ${ }^{200}$. Composé de deux ou trois transplanteurs issus du Conseil de fondation, cet organe permet à ses membres de se recentrer sur le traitement des problèmes structurels de la fondation et organisationnels de la transplantation. Dès la première séance, il est prévu d'y intégrer des représentants de chaque centre afin d'aborder des aspects médicaux entre experts ${ }^{201}$.

La volonté d'offrir au sein de la fondation un espace de travail décisionnel dévolu exclusivement aux transplanteurs afin d'y traiter les aspects médicaux et organisationnels de la transplantation joue un rôle important dans l'émergence de ce comité. Autour du chirurgien zurichois Largiadèr, qui après élection assume la charge de président, les membres du Comité exécutif commencent par définir clairement les besoins structurels de la fondation ${ }^{202}$. La mise sur

198 P-V CF, 10 mars 1987, p. 3.

199 P-V Comité exécutif (CE), 28 février 1991, p. 2.

200 P-V CF, 12 juin 1990, p. 7.

201 P-V CE, 7 mars 1990, p. 1 [notre traduction].

202 Idem, p. 2. 
pied d'un secrétariat central représente la première de ses priorités. Sans base pour assurer le fonctionnement administratif de la fondation, il est difficilement envisageable, pour les membres du Comité, de mener des actions concrètes pour favoriser la transplantation ou le don d'organes. Si le fonctionnement administratif de la fondation doit être assuré, le Comité juge absolument nécessaire de développer les activités concrètes de la fondation dans le domaine de la gestion des aspects organisationnels de la transplantation ${ }^{203}$. Le besoin le plus urgent est la mise en place d'un service de coordination des activités de transplantation à l'échelon national dont le but est de permettre une meilleure répartition des organes disponibles ${ }^{204}$. Le manque d'organisation de la transplantation en Suisse est considéré comme l'une des causes majeures de la pénurie d'organes dans les centres. Adrien Rohner de l'hôpital cantonal de Genève le fait déjà remarquer lors d'une séance du Conseil de fondation en 1989 en relatant une situation à laquelle il a été confronté:

«Un enfant qui attendait un foie durant 4 mois a été transféré à Louvain: en 10 jours un foie a pu être trouvé. On doit tout de suite unifier et organiser la coordination des centres ${ }^{205}$.»

Le problème de répartition concerne essentiellement la mise à disposition d'organes prélevés par un centre qu'il ne peut pas transplanter à l'un de ses patients. Jusqu'au début des années 1990, la pratique veut en effet que le centre où le donneur décédé est pris en charge soit prioritaire pour la réalisation de la transplantation. Pour des raisons médicales ou logistiques, les organes ne sont pas toujours utilisables sur place, surtout lorsqu'il s'agit de centres ne pratiquant que les greffes rénales. Souvent, les organes ne sont ni prélevés ni proposés aux autres centres. Limités dans leur activité par le seul recours aux prélèvements effectués sur place, les centres dans lesquels tous les types de transplantations sont effectués se sentent lésés. Les programmes de transplantation hépatique et cardiaque sont particulièrement dépendants d'une utilisation optimale

203 P-V CF, 12 juin 1990, p. 7.

204 P-V CF, 23 juin 1992, p. 3.

205 P-V CF, 5 décembre 1989, p. 2 [notre traduction]. 
de l'ensemble des ressources identifiées. Certains transplanteurs souhaitent vivement changer ce mode de fonctionnement et que ces organes soient mis à disposition des autres centres pour permettre une intensification des pratiques. Plus difficilement transplantables que le rein, le cœur et le foie doivent faire l'objet d'identification systématique et d'échanges sur l'ensemble du territoire.

L'élaboration du projet de coordination nationale met en jeu la collaboration de différentes entités liées à la transplantation. C'est au sein d'un groupe de travail de la Société suisse de chirurgie qu'un système informatique de centralisation des receveurs en attente de chaque organe est progressivement mis sur pied fin 1990 grâce au financement offert par l'hôpital cantonal de Genève ${ }^{\mathbf{2 0 6}}$. Les modalités de prise en charge financière par l'établissement genevois et de la collaboration entre la société de chirurgie et Swisstransplant ne sont toutefois pas clairement documentées. Rohner, membre du Comité exécutif comme de la société de chirurgie et professionnellement actif dans l'établissement genevois, joue un rôle décisif. En l'absence de reconnaissance politique accordée à Swisstransplant, cette phase d'élaboration des structures est caractérisée par des actions isolées sans politique de développement bien établie.

Si la base matérielle informatisée est mise à disposition par l'hôpital genevois, la gestion concrète de ce système de coordination passe par la création de postes de travail fixes. Toujours limité par le manque de moyens financiers excluant la prise en charge fixe et permanente de frais, le Comité exécutif adresse une demande de financement, au nom de Swisstransplant, à la CDS. Cette sollicitation ne permet guère d'espérer un financement direct, mais représente un moyen certain d'exercer une pression sur les différents gouvernements cantonaux ou instances fédérales. Pour Swisstransplant, l'implication de l'État dans la prise en charge des coûts liés au développement logistique et organisationnel de la transplantation est perçue comme indispensable. C'est ce qu'évoque Largiadèr lors d'une séance du Comité exécutif en juin 1990: «L'organisation de base pour la répartition et l'échange des organes et la gestion de la

206 P-V CE, 8 novembre 1990, p. 1-2. 
banque de données devraient être assumées par les cantons, alors que la fondation devrait prendre en charge les frais scientifiques ${ }^{\mathbf{2 0 7}}$. »

La sollicitation financière d'instances politiques marque une étape importante dans la définition du rôle que Swisstransplant souhaite assumer. La fondation se présente officiellement dans la sphère politique comme l'institution chargée de régler l'organisation de la transplantation et la promotion du don d'organes. Au nom de l'expertise médicale et gestionnaire de ses membres aux profils professionnels divers, Swisstransplant s'affiche comme l'institution de référence. La présentation de son fonctionnement et l'expérience acquise et accumulée dans les activités d'information du public depuis plusieurs années contribuent à renforcer ce constat. Il n'est plus question de rechercher des fonds ponctuels en organisant des manifestations publiques, mais d'impliquer désormais les pouvoirs publics. Bien que la demande à la CDS ne reçoive pas de réponse favorable ${ }^{208}$, elle n'en constitue pas moins une première tentative visant à acquérir une légitimité et une reconnaissance par les autorités publiques.

Si dans un premier temps la mobilisation du milieu politique n'aboutit pas par la voie officielle, la fondation peut néanmoins compter sur un réseau de relations informelles diffus. La diversité professionnelle des membres de son Conseil de fondation permet de démultiplier les demandes de financement aussi bien auprès des milieux politiques qu'industriels. À cet égard, Guy-Olivier Segond, à la tête du département genevois de la santé dès 1989 et membre du Conseil de fondation depuis 1990, sollicite la contribution de l'État de Genève qui accepte de prendre en charge les frais inhérents à la mise en place de la coordination sous forme d'une subvention spé-

207 P-V CF, 12 juin 1990, p. 7.

208 La CDS décide d'attendre un complément d'information sur le développement médicotechnique prévisible dans le domaine des transplantations ainsi que sur le cadre légal en vigueur dans les différents cantons avant de s'engager. Elle prévoit en outre de traiter les questions liées à la transplantation dans le cadre des discussions sur la médecine de pointe initiées au début de l'année 1992 entre les représentants des cantons en charge d'un hôpital universitaire. Voir Correspondance, "Exigences applicables aux établissements autorisés à effectuer des transplantations d'organes", 29 septembre 1992, p. 1-2. 
ciale jusqu'en $1995^{209}$. Initialement provisoire et destinée à la mise en place de la Coordination nationale dans l'attente d'un accord intercantonal, la subvention est repourvue d'année en année jusqu'à la fin de la période envisagée dans ce chapitre, à savoir l'année $2000^{210}$.

\subsection{Mise en place de la Coordination nationale}

\section{Autonomiser la coordination des transplantations}

Avant même de jeter les bases de l'unification des pratiques de répartition des organes, la Coordination nationale, dont l'ouverture officielle en date du $1^{\text {er }}$ octobre 1992 fait l'objet d'une conférence de presse, conduit à une réorganisation structurelle de Swisstransplant. Le financement genevois permet en effet la création du secrétariat général qui prend désormais en charge les activités administratives de la fondation et la gestion des données concernant la transplantation rénale réalisées jusque-là par le secrétariat du laboratoire d'histocompatibilité ${ }^{211}$.

Concrètement, la mise en place de la Coordination nationale génère la création de deux postes que le financement genevois permet de repourvoir. Largement impliqué dans la prise en charge du matériel informatique nécessaire à la centralisation et au partage des données, l'hôpital cantonal de Genève met également à disposition gratuitement des locaux pour les coordinateurs ${ }^{\mathbf{2 1 2}}$.

Si l'utilité d'une telle structure dans la gestion de la transplantation ne fait aucun doute pour l'ensemble des membres de la fondation, son incorporation à l'organigramme suscite des tensions. Confiée au Comité exécutif, cette incorporation suppose une

209 Le Temps, 02 juin 1998, www.letemps.ch/suisse/1998/06/16/geneve-guy-oliviersegond-renonce-guide-collaboration-intercantonale (consulté en 2019); P-V CE, 18 décembre 1992, p. 3.

210 P-V CE, 3 novembre 1994, p. 5.

211 P-V CF, 6 avril 1993, p. 5.

212 P-V CE, 18 décembre 1992, p. 1-2. 
redéfinition des relations hiérarchiques entre les différents organes constitutifs de Swisstransplant. C'est surtout l'influence grandissante prise par le Comité exécutif dans la gestion des activités de la fondation qui soulève des divergences. En effet, le principal grief que la réorganisation entraîne est la proposition de subordonner la Coordination nationale au Comité exécutif ${ }^{213}$.

Le risque de créer un lien de subordination entre la structure responsable de la répartition des organes et le Comité exécutif, composé de transplanteurs et de médecins, suscite des craintes. Si la séparation stricte entre processus de gestion des organes et pratique des transplantations est effective dans les hôpitaux, elle devrait également être visible dans l'organisation structurelle de la fondation, selon les vœux de certains membres. La remise en cause de ce lien hiérarchique, d'abord sous la forme d'une opposition de principe, s'aiguise lors des discussions qui entourent sa mise en place concrète. Les oppositions les plus nourries surgissent lors de la création d'un poste de surveillance des activités de coordination confié à Gilles Mentha, chirurgien à Genève au sein de la clinique de chirurgie viscérale ${ }^{214}$. Émanant de certains médecins du Comité exécutif, soucieux de donner une image claire des différents processus médicaux et organisationnels de la transplantation, les contestations soulignent la nécessité d'une séparation rigoureuse des intérêts médicaux et organisationnels. Si la désignation d'un chirurgien transplanteur fragilise la probité des activités de la fondation, elle est surtout perçue comme une menace pour la répartition équitable des organes entre les centres.

Ce conflit d'intérêts pousse Jeannet, qui souhaite subordonner la Coordination au laboratoire de référence pour l'histocompatibilité de Genève, à solliciter Eurotransplant pour connaître les usages à l'étranger. Les pratiques de cette institution établissent clairement une séparation structurelle entre coordination et transplantation, comme l'illustre la réponse transmise lors d'une séance du Comité pour information:

213 Idem, p. 4.

214 Idem, p. 3. 
«Dans toutes les organisations européennes de transplantation, la distribution des différents organes lorsqu'un donneur est disponible est assurée par le bureau central ou le secrétariat général de l'organisation. La direction de ce dispositif central est souvent assumée par un immunologiste mais jamais par un chirurgien ${ }^{215}$. "

Un groupe formé par certains médecins et chirurgiens lausannois rejoint le modèle défendu par l'immunologue genevois mettant l'accent sur l'absence de chirurgiens transplanteurs au sein des structures chargées de répartir les organes «dans tous les autres pays ${ }^{216}$ ».

L'autre point épineux qui divise les chirurgiens est la localisation de la structure nouvellement créée au sein même de l'hôpital cantonal genevois. La crainte d'une collusion d'intérêts entre le centre de transplantation de Genève et la Coordination nationale, déjà soulevée par l'attribution de la surveillance des activités à un chirurgien local, se trouve renforcée. Danko Sege du département de chirurgie de Saint-Gall préférerait un lieu neutre dans des locaux indépendants à Berne par exemple ${ }^{217}$. Le risque d'opacité et de mélange des responsabilités qu'un lien trop ténu entre l'hôpital genevois et la Coordination nationale pourrait induire nécessite également une clarification et une séparation d'aspects relevant de la logistique. Le numéro de téléphone qui doit permettre de joindre les membres de la Coordination pour annoncer un donneur, par exemple, est jugé problématique. Il transite par l'hôpital, ce qui peut semer la confusion aux yeux du public en laissant supposer un contrôle de l'établissement sur les activités de coordination ${ }^{218}$.

Rassemblés autour du président du Comité Philippe Morel, les partisans d'une collaboration rapprochée entre la Coordination et le Comité exécutif jugent ces suspicions infondées. Selon eux, la création de la Coordination vise justement à empêcher la répartition et les échanges informels d'organes. Le fonctionnement même de

215 Correspondance, lettre d'Eurotransplant, 2 décembre 1992, p. 1 [notre traduction].

216 Correspondance, [département de médecine interne, division de cardiologie], 10 décembre 1992.

217 P-V CE, 18 décembre 1992, p. 4.

218 Idem, p. 5. 
la Coordination qui répartit les organes selon des règles établies et reconnues représente un rempart contre l'arbitraire. Claires et simples, les règles doivent permettre, comme le pense le chirurgien zurichois Rolf Schlumpf, de "faire disparaître cette crainte, puisqu'alors il ne sera plus possible d'avoir des situations équivoques $^{219}{ }$.

Pour pallier tout risque éventuel, il est prévu de charger la Coordination de transmettre des rapports d'activité mensuels à l'ensemble des membres du Comité et aux centres de transplantation. Cette mesure est jugée largement suffisante pour mettre la fondation et les centres définitivement à l'abri de conflits d'intérêts et empêcher toute pression exercée pour l'obtention d'organes. Les critiques émises sur la localisation n'ont dès lors plus lieu d'être. Aux yeux de Morel, il convient plutôt d'être reconnaissant envers la générosité de l'État genevois et de son hôpital, qui financent la mise en place de la Coordination et l'ensemble du matériel, épargnant ainsi à Swisstransplant des recherches de fonds et les sacrifices financiers induits par un loyer ${ }^{220}$. Fort de ces arguments, le président obtient une majorité qui entérine, sous réserve de l'approbation du Conseil de fondation, la subordination de la Coordination au Comité exécutif dès le mois de septembre $1992^{221}$.

\section{La promotion du don dans l'espace public}

Après la dissolution du groupe de travail Swisstransplant-Lions Club en 1991, les activités de promotion du don et d'information sur la transplantation reviennent au Comité exécutif. Exécutant du Conseil de fondation selon la définition des liens hiérarchiques qui unissent ces deux organes, il poursuit le façonnement de la politique de développement de la fondation. Si les fonds récoltés grâce au partenariat avec le Lions Club étaient dévolus exclusivement à l'information du public, ce secteur d'activité est désormais dépourvu de sources de financement. À la charge exclusive de la fon-

219 Idem, p. 4.

220 Idem, p. 5.

221 P-V CE, 4 septembre 1992, p. 6-7. 
dation, les structures mises en place pour assurer la visibilité dans la sphère publique sont repensées pour limiter les frais. La centrale d'information de Schaffhouse est fermée alors que les activités qui y étaient assumées sont confiées au secrétariat général, à Genève. Pour permettre une séparation entre les activités d'information et organisationnelles, la centrale téléphonique reste en service ${ }^{222}$. Il s'agit d'éviter une surcharge de travail qu'entraînerait la prise en charge des appels directs par la Coordination.

Sans ressources fixes pour créer des programmes d'information ou de nouvelles structures dévolues uniquement à la promotion du don, le Comité exécutif mise, dès 1994, sur l'organisation de la Journée nationale consacrée à la transplantation et au don d'organes $^{223}$. Si la réalisation de ce type de manifestation exige de larges moyens financiers, leur caractère ponctuel facilite le démarchage de plusieurs sponsors. Les sollicitations sont dirigées essentiellement vers l'industrie pharmaceutique dont on constate la large propension à soutenir le développement de la fondation. L'intégration dès 1992 du vice-directeur de Sandoz-Pharma au sein du Conseil de fondation dès la création de Swisstransplant permet de bénéficier d'un lien direct avec l'entreprise bâloise. Entre défense de l'indépendance de la médecine et garantie matérielle pour la promotion du don, Swisstransplant manifeste la volonté de rester discrète sur la relation qu'elle entretient avec l'industrie pharmaceutique. Ainsi, s'il est unanimement reconnu que l'organisation de ce type d'événements serait irréalisable sans les moyens financiers mis à disposition par Sandoz-Pharma ${ }^{224}$, le vice-directeur de l'entreprise bâloise indique que «les industries pharmaceutiques ne devraient pas apparaître clairement dans le comité d'organisation, l'impact pouvant être négatif aux yeux du public ${ }^{225}$ ».

222 P-V CF, 6 avril 1993, p. 8.

223 La première Journée nationale pour la transplantation et le don d'organes a lieu le

11 septembre 1994. Depuis, une journée nationale est organisée chaque année dans le courant du mois de septembre.

224 P-V CF, 29 avril 1994, p. 5.

225 Idem, p. 5. 
L'intérêt de la Journée nationale, mise sur pied pour attirer l'attention sur le don d'organes et la transplantation, réside principalement dans son impact médiatique. La forme que prennent ces rassemblements, dans lesquels l'implication de politiciens et de patients transplantés est centrale, en est largement influencée. Afin de susciter l'intérêt des médias, la participation de représentants des instances politiques est particulièrement recherchée. Dans le cas de la Journée européenne mise sur pied par Swisstransplant en 1997, il s'agit même de centrer une partie de la manifestation uniquement sur un débat concernant le problème du commerce d'organes auquel sont conviés des politiciens de l'Europe entière. Placée sous le patronage du Conseil de l'Europe, cette manifestation donne une reconnaissance officielle aux problématiques qui y sont traitées ${ }^{226}$. Pour démontrer concrètement l'apport thérapeutique incontestable de la transplantation, la participation de patients transplantés à ces manifestations constitue un élément essentiel. Une partie centrale de l'événement leur est généralement consacrée sous forme de rassemblements et d'actions symboliques variées. Ces manifestations, en plus du potentiel promotionnel qu'elles revêtent, permettent surtout de transmettre une image positive de la transplantation et du don d'organes ${ }^{227}$.

L'évaluation de la réussite de la Journée nationale se mesure largement à l'aune de la couverture médiatique dont elle a été l'objet. Même si tout article de presse sur la transplantation représente un succès constitutif du processus plus général de diffusion d'information, la couverture médiatique en lien direct avec la journée reste une priorité pour Swisstransplant. Dans le cadre de la deuxième Journée nationale en 1995, Morel, alors président du Comité exécutif, l'explique dans le compte rendu qu'il propose lors de la réunion du Comité en septembre:

«Le vol offert aux enfants transplantés à Zurich n'a pas eu la répercussion médiatique espérée puisqu'aucun journaliste n’a parti-

226 P-V CF, 2 juin 1995, p. 3-4.

227 Cette préoccupation est également partagée par les milieux associatifs, comme nous le développons dans la seconde partie de l'ouvrage. 
cipé au vol. Cependant par cette journée, de nombreux reportages sur des patients transplantés ont été publiés dans l'ensemble des médias suisses ${ }^{228}$.»

La médiatisation des événements est particulièrement importante pour la fondation. Dans le discours qu'elle en fait rétrospectivement lors de réunions, la diffusion de cartes de donneurs et de brochures est directement corrélée à l'importance de la couverture médiatique de la manifestation. La première Journée nationale est dans cette optique une réussite comme le relate Philippe Morel devant le Conseil de fondation en novembre 1994 :

"La répercussion de cette dernière a été excellente puisque l'événement a été repris par le téléjournal suisse romand et suisse italien, qu'elle a suscité plus de 200 articles dans la presse, et a eu pour conséquence la distribution de 55000 brochures d'information et 30000 cartes de donneur ${ }^{229}$."

Chaque année, le Comité exécutif s'efforce également d'organiser des conférences de presse. Elles sont l'occasion de présenter le travail de la fondation et les statistiques concernant la transplantation. Avec la mise en place de la Coordination nationale, elles prennent une importance nouvelle dans la divulgation d'information concernant l'ensemble des chiffres entourant la pratique des transplantations en Suisse. Les conférences de presse sont désormais l'unique moment dans l'année où la fondation présente les chiffres. Pour compléter l'offre informative consultable à tout moment, la fondation décide de publier un rapport d'activité spécifique pour le public, en complément du rapport transmis au corps médical. L'activité médicale des centres de transplantation est ainsi associée à Swisstransplant. Dans l'optique d'une constante recherche de reconnaissance et de financement, certains acteurs politico-sanitaires sont des destinataires privilégiés; les directions d'hôpitaux, les assureurs, les directeurs de la santé des vingt-six cantons et les conseillers nationaux reçoivent d'office ce rapport ${ }^{230}$.

228 P-V CE, 20 septembre 1995, p. 4.

229 P-V CF, 3 novembre 1994, p. 2.

230 P-V CE, 30 janvier 1998, p. 2. 
Le potentiel médiatique des conférences de presse fait également l'objet d'une définition stratégique des éléments qui doivent les constituer. Comme dans le cadre des "Journées », la présence de politiciens est activement recherchée. L'association d'une personnalité politique représente un moyen promotionnel influent, comme l'illustrent les propos du président du Conseil de fondation Segond en 1995 :

«Lors de la conférence de presse de Swisstransplant du 4 avril, qui marquait les 10 ans de la fondation, Mme Ruth Dreifuss a montré sa carte de donneur devant les caméras, une image qui a été rediffusée à de nombreuses reprises depuis lors ${ }^{231}$.»

Si la participation de patients est également un élément constitutif des conférences de presse, les situations médicales émotionnellement chargées permettent d'obtenir une large couverture médiatique ${ }^{\mathbf{2 3 2}}$. Le président de la fondation souligne lors de la conférence de presse annuelle de 1997 «l'importance et l'impact qu'ont eu les témoignages d'un enfant transplanté et de sa mère, donneuse de rein, ainsi que la présence d'une deuxième mère avec son enfant transplanté, lors de cette conférence de presse qui a trouvé un large écho dans la presse écrite avec des articles dans 83 journaux en Suisse allemande, 20 journaux en Suisse romande et 8 journaux au Tessin ainsi que dans un journal romanche ${ }^{233}$ ».

Parallèlement à ces manifestations ponctuelles, des actions plus diffuses sont organisées. Sous l'appellation de «campagnes nationales d'information ", elles gardent un caractère de promotion du don, mais elles sont plutôt centrées sur la diffusion d'information sans visée médiatique prioritaire. Ces initiatives, plus modestes et réalisées localement, sont directement centrées sur le grand public sans passer par les médias. En 1998 par exemple, des messages d'informations sont affichés sur les autobus à Genève alors que la diffusion de brochures est effectuée dans les écoles de recrues ${ }^{\mathbf{2 3 4}}$. Il arrive que des organismes sans lien avec la fondation proposent

231 P-V CF, 2 juin 1995, p. 2.

232 Sur la dimension émotionnelle de la cause du don d'organes, voir aussi chapitre 8.

233 P-V CF, 25 avril 1997, p. 9.

234 P-V CE, 7 novembre 1997, p. 4. 
de se charger de l'organisation logistique et de la prise en charge financière des activités. En 1997 par exemple, la Jeune chambre économique suisse offre de prendre en charge une vingtaine de séances d'information dans lesquelles interviennent des chirurgiens ${ }^{\mathbf{2 3 5}}$.

\section{La promotion du don à l'hôpital}

L'identification de donneurs potentiels représente la première étape dans le processus médical qui conduit à la réalisation d'une transplantation. Le nombre de donneurs apparaît comme insuffisant dès les premières réunions du Conseil de fondation. Pour les membres de la fondation, la cause principale du manque de donneurs réside dans le manque de motivation du personnel médical concerné ou d'information sur la transplantation et le processus d'identification des donneurs. Largiadèr en fait mention en 1985 lors d'une réunion à propos de la transplantation rénale: «Le don de rein est maintenant bien accepté par la population, mais le manque d'organes provient plutôt du manque d'organisation des médecins, en particulier dans les hôpitaux de districts ${ }^{236}$. » Dans un contexte d'émergence et d'élaboration structurelle, peu d'actions directement centrées sur l'identification des donneurs dans les hôpitaux sont entreprises. Les initiatives mises en place visent plutôt à évaluer l'ampleur de ce phénomène par le biais d'enquêtes réalisées auprès de médecins ou dans les hôpitaux. Évoquées sans faire l'objet de présentation approfondie, elles sont menées par certains transplanteurs à titre privé, sans implication de la fondation ${ }^{237}$. L'information du public reste l'objet principal des efforts déployés, en espérant des retombées indirectes sur le personnel des hôpitaux.

Les premières mesures ciblées débutent par l'envoi de documentation dans les services de soins intensifs. Des procédures unifiées pour l'identification de donneur sont par exemple distribuées dans ces unités ${ }^{238}$. Pour parvenir à une utilisation efficiente des ressources

235 P-V CF, 29 avril 1994, p. 5.

236 P-V CF, 4 décembre 1985, p. 2.

237 P-V CF, 7 décembre 1988, p. 2.

238 P-V CE, 4 septembre 1992, p. 7. 
disponibles, il apparaît central, pour les membres de la fondation, d'intervenir au cœur du processus d'identification. Il s'agit donc d'actions ponctuelles qui visent à informer et à faire de l'identification de donneurs un acte médical à envisager dans des situations bien précises. Ciblées par type de profession, ces brochures permettent d'informer, mais également de responsabiliser le personnel soignant qui, particulièrement dans les unités de soins intensifs, entretient un contact direct avec des donneurs potentiels.

Si l'envoi de brochures informe et répand un modèle technique que le personnel est invité à suivre, la modification concrète des pratiques passe par un changement initié de l'intérieur des services eux-mêmes. L'organisation médicale de la transplantation entre les hôpitaux est façonnée par le biais des groupes de travail et des structures dirigeantes de la fondation, mais le fonctionnement interne des services, hors du cercle des transplanteurs, est plus difficilement atteignable. Dans l'optique de modifier les pratiques de l'intérieur, la fondation décide de participer au "programme européen d'éducation pour les hôpitaux ayant des donneurs d'organes ${ }^{239}$ ".

Créé pour les médecins et le personnel soignant et développé en Angleterre dans l'optique d'être appliqué dans l'ensemble de l'Europe, il a pour but de présenter des techniques pour formuler les demandes de greffes ${ }^{240}$. La mise en commun de ressources éducatives entre les différentes institutions impliquées dans la transplantation passe par l'élaboration de pratiques standardisées et de stratégies communes pour promouvoir le don d'organes. Les participants, désignés par chaque pays intéressé, sont invités à suivre une formation à Leiden au siège d'Eurotransplant ${ }^{241}$. Il s'agit d'y acquérir un savoir spécifique à diffuser par la suite dans le cadre de séminaires organisés à un rythme encore indéfini. Pour s'assurer d'une diffusion suffisamment large au sein de la sphère médicale en Suisse, le secrétaire de l'antenne suisse du programme

239 Correspondance, "European Donor Hospital Education Program", 19 février 1991.

240 Idem.

241 Correspondance, "European Donor Hospital Education Program EDHEP", 25 novembre 1992. 
parvient à obtenir la reconnaissance de ce séminaire dans le cadre de formation continue ou postgraduée de la Société de médecine intensive ${ }^{242}$. Si elle donne une valeur institutionnelle à la formation proposée, cette reconnaissance offre surtout la garantie d'un public cible régulièrement renouvelé.

À l'exception d'un médecin modérateur, les premiers participants désignés pour suivre cette formation durant l'été 1993 par Swisstransplant sont professionnellement responsables de la coordination à l'échelon national ou dans des centres de transplantation $^{243}$. La fonction de coordination n'est pas formellement liée à l'organisation du prélèvement sur place dans les hôpitaux. Certains établissements possèdent même leur propre personnel à qui est dévolue cette charge. C'est le cas de l'hôpital de Berne comme l'explique Christian Seiler:

«[...] le centre de Berne envoie sa coordinatrice locale dans les hôpitaux périphériques lorsqu'il y a des prélèvements; elle agit donc sur place pour organiser la coordination. Cette mission pourrait également être confiée aux Coordinatrices nationales si la logistique le permettait ${ }^{244}$.»

Si l'implication de la Coordination nationale dans le processus de prélèvement est réalisée progressivement sans suivre un plan d'action défini avec précision, elle signifie un changement dans l'appréhension du problème du manque d'organes. Qu'il soit causé par le manque de motivation des hôpitaux ou pour une hausse du refus de donner aux causes inconnues, les efforts se centrent sur le développement des capacités d'acteurs acquis à la cause de la transplantation et dotés d'un savoir-faire spécifique validé par des formations. "Il serait souhaitable que les demandes de don d'organes soient faites par des professionnels $^{245}$ », affirme le représentant de Sandoz au Conseil de fondation.

242 Correspondance, "EDHEP-votre courrier 9 juin 1997 », 17 juin 1997.

243 Correspondance, "European Donor Hospital Education Program EDHEP", 25 novembre 1992.

244 P-V CE, 18 décembre, 1992, p. 13.

245 P-V CE, 29 avril 1994, p. 7. 


\subsection{Professionnalisation et anticipation des changements politiques}

La nécessité d'entreprendre des modifications menant à une professionnalisation de Swisstransplant est reconnue par l'ensemble des membres du Comité exécutif dès le milieu des années 1990. Le développement des activités organisationnelles entraîne une charge de travail importante pour le Comité exécutif, présentée parfois comme un frein à la motivation d'implication des chirurgiens dans la fondation. Au sein des centres de transplantation, il devient difficile de recruter des représentants pour siéger au Comité exécutif, comme l'explique le chirurgien Amédée Genton de Lausanne: «Le but à long terme, ceci est évident, est la professionnalisation de Swisstransplant, car tous les membres actuels sont surchargés et il est très difficile de trouver des volontaires ${ }^{246}$.»

Si ces aspects fonctionnels jouent un rôle dans la volonté de professionnaliser les structures, c'est surtout l'émergence du processus législatif régulant la pratique de la transplantation qui pousse les membres à envisager cette éventualité. Initiée dès 1995 par une commission de travail ${ }^{247}$, l'élaboration de la future loi fédérale représente pour la fondation le début d'une négociation sur le rôle que les autorités fédérales lui attribueront dans la nouvelle organisation de la transplantation. Certains membres souhaitent anticiper l'application de la loi pour permettre à la fondation de se positionner avantageusement dans les négociations qu'elle devra entreprendre avec la Confédération. Sous la forme qui est la sienne, la fondation semble cependant mal engagée. Ce constat est renforcé par la recherche d'un nouveau président en 1998, à la suite de la démission du conseiller d'État genevois Segond. Les entretiens préliminaires, menés par Largiadèr avec plusieurs conseillers nationaux et aux États, mettent en lumière la nécessité unanime d'adapter les structures sans donner toutefois plus de détails ${ }^{248}$.

Dans ce contexte, Largiadèr réfléchit à un nouveau modèle de structures dirigeantes pour trouver un président influent dans la

246 P-V CF, 15 avril 1996, p. 2.

247 P-V CE, 29 novembre 1995, p. 6

248 Protokoll, "Zweite Sitzung der Findungskommission Swisstransplant», p. 1. 
sphère politique fédérale. À la tête de la commission composée de médecins du Comité exécutif et du Conseil de fondation qui se réunit à deux reprises les 19 août et 28 septembre 1998 pour superviser la recherche d'un nouveau président, le chirurgien zurichois propose un modèle centré sur la professionnalisation des structures dirigeantes.

Resté au stade de projet, ce modèle suggère la dissolution du Comité exécutif au profit d'un comité directeur constitué d'un seul représentant du corps médical, du président de la fondation, d'un vice-président, d'un représentant de la Coordination nationale et d'un secrétaire ${ }^{249}$. Il ne s'agit plus de se réunir pour préciser l'organisation de la transplantation, mais bien de gérer les activités de la fondation comme un système organisationnel établi et fixé. Dans cette optique, l'omniprésence de médecins à la tête de la fondation n'est plus justifiée dans le nouveau modèle qui présente l'organisation de la fondation. Cette volonté affichée de diminuer l'influence du Comité exécutif est mal perçue par la majorité des membres du Comité exécutif. Le flou qui entoure le statut qu'obtiendra la fondation dans le cadre de l'application de la future loi fédérale incite à considérer tout changement comme prématuré. La prise de décision précipitée est envisagée comme une mise en péril de l'équilibre médical établi. Le président du Comité exécutif Philippe Morel l'évoque dans un courrier transmis à Largiadèr en tant que responsable de la commission de recherche d'un président:

«Les modifications des structures devraient en effet s'effectuer au moment où notre Fondation se placera sous la responsabilité directe des instances fédérales compétentes [...]. Le bon fonctionnement actuel, au plan médical de notre fondation, l'entente qui règne entre les différents partenaires; la qualité des prestations que nous offrons pourraient en effet souffrir sévèrement des modifications intempestives de notre structure ${ }^{250}$.»

Malgré la volonté de temporisation exprimée par certains membres du Comité exécutif, le Conseil de fondation accepte sans

249 Correspondance, "Organisation und Arbeitsweise der Stiftung Swisstransplant", 23 septembre 1998

250 Correspondance, sans titre [avis du conseil exécutif sur les modifications des structures de la fondation], 14 octobre 1998. 
discussion, conjointement au changement de présidence en 2000, de profondes modifications de l'organigramme.

Derrière les transformations structurelles entreprises apparaît en filigrane une "démédicalisation" des structures dirigeantes, caractérisée par la dissolution du Comité exécutif et son remplacement, comme le prévoyait le projet élaboré en 1998, par un Comité directeur composé de cinq membres issus du Conseil de fondation, dont un seul est médecin, et du président. À chaque membre correspond un département dont il est l'unique porte-parole. Selon ce redécoupage des responsabilités, les postes clés doivent à moyen terme faire l'objet d'une professionnalisation.

Le Comité médical, créé sur les bases de l'ancien Comité exécutif ${ }^{251}$, est composé uniquement de représentants des centres de transplantation. Son activité est limitée au traitement de problèmes scientifiques et médicaux, comme l'élaboration d'un accord entre la fondation et Eurotransplant sur la traçabilité des organes échangés $^{252}$. La gestion de la coordination qui représentait une des tâches importantes de l'ancien Comité exécutif est attribuée à des groupes de travail ${ }^{253}$. La représentation du Comité médical au Conseil de fondation est limitée à un seul membre, changement qui symbolise la gestion centralisée de la transplantation et la capacité de décision réduite des hôpitaux.

La dissociation entre les aspects médicaux et l'organisation logistique de la transplantation est mal perçue par le corps médical de la fondation. L'indépendance du lien entre la Coordination nationale et le nouveau Comité médical contribue à fragiliser le contrôle médical sur la transplantation. Si les aspects spécifiquement organisationnels de la répartition des organes peuvent être réalisés en dehors de toute relation avec le corps médical, d'autres problèmes, comme l'évaluation ou l'adaptation des règles d'échanges, nécessitent selon eux la consultation des transplanteurs ${ }^{254}$. La prise en charge de ces aspects

251 P-V CF, 13 janvier 2000, p. 2.

252 P-V Comité médical (CM), 25 juin 2001, p. 2.

253 P-V Comité directeur (CD), 14 août 2000, p. 2.

254 Correspondance, "Réunion du comité médical du 28 janvier 2000 ", 8 février 2000, p. 2. 
par les groupes de travail renforce encore la séparation, puisqu'ils se rassemblent lors de leurs séances de travail indépendamment les uns des autres, selon une logique d'organe. Pour les membres du Comité médical, le fonctionnement de la coordination nécessite par définition un partage général des informations et des problèmes médicaux rencontrés dans la pratique.

La diminution des possibilités d'intervention du corps médical dans l'ensemble des nouvelles structures est également dénoncée. L'intégration d'un seul médecin dans le Comité directeur est jugée largement insuffisante, tout comme la limitation à un unique représentant du Comité médical dans le Conseil de fondation. Pour les membres du Comité, cette amputation rend difficiles la possibilité et la liberté d'exprimer le point de vue médical au sein de la fondation ${ }^{255}$. Dans un courrier adressé à la nouvelle présidente Trix Heberlein, le président du Comité exécutif conteste ce processus qui tend à isoler les médecins hors des structures dirigeantes:

«En ces périodes de turbulences législatives, il est important de renforcer la cohésion, l'unité et la synchronisation des activités médicales de Swisstransplant ${ }^{256}$.»

L'évaluation du contexte politique et des stratégies pour s'y adapter ne fait pas l'unanimité parmi les membres. C'est justement l'incertitude autour de l'avenir de la fondation qui impose, pour les partisans de ce nouveau modèle moins monopolisé par les médecins, des modifications structurelles, validées par le Conseil de fondation.

Une dernière mesure mal comprise par le corps médical dans le cadre de ces restructurations est la suppression du Club de Transplantation ${ }^{257}$. Créé en 1993, il est décrit comme «un forum d'accès libre auquel peut participer l'ensemble des médecins intéressés à la transplantation, quelle que soit leur spécialitée 258 ». Le but de cette organisation est de permettre à la fondation de diffuser la thématique de la transplantation en dehors de la sphère limitée des

255 P-V Comité médical (CM), 11 septembre 2000, p. 2.

256 Correspondance, "Organigramme de Swisstransplant", 8 février 2000, p. 2.

257 P-V CF, 4 décembre 2000, p. 3

258 P-V CE, 4 septembre 1992, p. 3. 
transplanteurs. Subordonné informellement au Comité exécutif qui en supervise le fonctionnement et les activités scientifiques, il permet d'élargir la diffusion de la thématique à un public médical plus large, tout en gardant le contrôle sur l'espace scientifique.

La raison de cette dissolution n'est pas explicitement abordée, mais on peut penser qu'elle trouve son origine dans l'opacité des liens qui l'unissent à la fondation. Organisation fondée par des transplanteurs, ses membres sont parfois intégrés informellement au Comité exécutif rendant les liens entre le Club et les structures dirigeantes mal définis. Dans une perspective générale de clarification des statuts et des rapports hiérarchiques entre les structures, les liens paraissent trop intriqués pour être préservés. Alors que le corps médical se sent mis de côté, la perspective d'une bureaucratisation de Swisstransplant induite par l'imminence de la loi fédérale sur la transplantation pousse les membres du Comité médical à se regrouper dans une structure externe (la Société scientifique de transplantation). Afin de pallier ce qui est perçu comme un manque de représentativité du nouveau Comité médical, cette société se veut ouverte à tous les spécialistes de la transplantation, notamment les membres de l'ancien Comité exécutif ${ }^{259}$. Si le Club de Transplantation avait été créé en 1993 pour offrir un accès à Swisstransplant à tous les médecins intéressés par la transplantation, c'est un phénomène inverse que l'on constate en 2000 avec la professionnalisation.

\subsection{Conclusion}

À la fin des années 1980, la structure de Swisstransplant est fortement marquée par l'influence des centres de transplantation. Le poids des transplanteurs dans l'organisation des pratiques s'en ressent largement. La volonté de la fondation de réguler la transplantation ne s'apparente en aucun cas à une remise en cause des pratiques médicales ou de la mainmise des médecins sur celle-ci. La création du Comité exécutif au début des années 1990 représente une étape clé du développement structurel de la fondation. La par-

259 P-V CM, 4 décembre 2000, p. 3. 
ticipation au Comité exécutif s'apparente alors à une appartenance communautaire. Composé de médecins impliqués dans le domaine de la transplantation, il donne une forte orientation médicale à la mise en place des structures organisationnelles de la fondation. Le développement de ses activités ne va pas sans révéler des tensions entre les membres. Issues d'opposition d'idées, parfois alimentées par des rivalités, ces tensions n'empêchent pas le Comité exécutif de poursuivre ses activités aussi bien dans la gestion des échanges d'organes que dans le domaine de la promotion du don.

L'implication prépondérante des médecins jusqu'à la fin des années 1990 est une adaptation pragmatique au contexte politique plus global dans lequel la fondation se développe. Le manque d'engagement des instances politiques, sollicitées à plusieurs reprises, contraint la fondation à mobiliser ses propres ressources à la recherche d'autres sources de financement et de soutien. C'est grâce à des appuis ponctuels que certaines étapes importantes du développement structurel et opérationnel de Swisstransplant ont été concrétisées (la collaboration avec le Lions Club qui a permis le lancement d'une carte de donneur et le financement de la Coordination nationale grâce à la subvention du canton de Genève).

La professionnalisation des postes clés au sein de Swisstransplant remet en question la prédominance des médecins dans la gestion de la fondation. Influencée par l'élaboration du processus législatif mené par la Confédération à partir du milieu des années 1990, cette professionnalisation pose un dilemme au corps médical. D’un côté, la nécessité de présenter une structure pour négocier une place de choix au centre du mécanisme de la nouvelle organisation de la transplantation apparaît comme inéluctable. De l'autre, la crainte de perdre un droit de regard et d'intervention privilégié, issu de la période d'émergence de la transplantation dans les hôpitaux, est perçue comme une remise en cause de leur autorité. Les tensions entre les membres de la fondation prennent une ampleur accrue à l'occasion de la modification de l'organigramme à la fin des années 1990. L'élaboration de la future loi fédérale redéfinit non seulement le fonctionnement de Swisstransplant, mais aussi les modalités de circulation des organes, comme le montrera le chapitre suivant. 


\section{Chapitre 3}

\section{L'organisation médicale et logistique de la transplantation: le rôle de Swisstransplant}

\section{Alexia Cochand}

Dès la mise en place des programmes de transplantation rénale à la fin des années 1960, l'échange d'organes représente un élément essentiel pour en garantir un nombre suffisant à chaque centre. Bien que des arrangements existent entre certains centres dès cette période, ils sont, compte tenu de leur caractère officieux, nécessairement instables et variables. L'activité de transplantation de chaque centre dépend ainsi d'abord de ses propres prélèvements. Regroupant aussi bien des transplanteurs que des acteurs politiques, Swisstransplant parvient au début des années 1990, comme l'a montré le chapitre précédent, à mettre en place une coordination nationale, cadre organisationnel régulant l'activité de transplantation dans l'ensemble de la Suisse. Alors que la circulation des organes s'appuyait sur des règles tacites et des arrangements informels entre médecins, elle acquiert progressivement une forme réglementée entre les centres.

Ce chapitre aborde les principales étapes de cette évolution majeure, jusqu'au moment où la régulation de la transplantation devient un objet de politique fédérale à part entière. Nous examinerons d'abord les outils développés par Swisstransplant pour réguler 
la transplantation d'organes et les conséquences de la formalisation des échanges sur les pratiques. Nous aborderons ensuite les moyens qu'elle a déployés pour résoudre la question sensible des transplantations réalisées en clinique privée. Enfin, nous nous intéresserons au rôle de la fondation dans la régulation des pratiques à l'intérieur du réseau constitué par les centres en nous arrêtant sur la question du type d'organes transplantables dans chaque centre.

La correspondance conservée dans les archives de la fondation représente la base documentaire principale sur laquelle ce chapitre est constitué. Composé de très nombreux documents émis entre Swisstransplant et les centres de transplantation, et entre les membres des différentes entités, ce corpus permet d'identifier plusieurs thèmes majeurs de par leur récurrence. Dans notre analyse, nous tiendrons aussi compte des silences autour d'autres problématiques révélatrices du rôle de Swisstransplant et de l'évolution organisationnelle du don d'organes.

\subsection{La répartition des organes avant la mise en place de la Coordination nationale}

La mise en place de programmes de transplantation dans les hôpitaux suisses pose dès la fin des années 1980 la question de la répartition des organes entre centres ${ }^{260}$. Les spécificités médicales de cette pratique, dont la réussite ne dépend pas uniquement de l'habileté chirurgicale des transplanteurs, mais également de facteurs biologiques, rendent nécessaire le transfert d'organes entre les différents centres. Il s'agit en effet d'assurer la réalisation de transplantations par une circulation optimale des ressources disponibles. L'élaboration des échanges suit un processus initial de collaboration informelle entre médecins. La mobilisation de réseaux de connaissances, de liens d'amitié ou d'affinité entre les chirurgiens transplanteurs et médecins impliqués dans la procédure médicale permet d'obtenir ou de transmettre des organes inutilisables dans

260 Dans ce chapitre, comme dans le précédent, le terme "répartition" renvoie à la circulation des organes prélevés entre les hôpitaux, alors que le terme "attribution" désigne la distribution des organes aux patients receveurs. 
le centre de prélèvement à une équipe de transplantation dans un autre centre.

Lors de la création de Swisstransplant en 1985, la transplantation rénale fait déjà l'objet d'une répartition organisée. Sous l'égide du groupe de travail ad $h o c$, un cadre normatif définit les échanges entre les établissements concernés. À cette époque, l'usage veut que les reins soient prélevés et transplantés dans le même centre. Si le rein prélevé ne peut, pour des raisons de compatibilité, être greffé sur place, il est proposé à d'autres hôpitaux. Dans cette optique, chaque centre transmet régulièrement une liste d'attente au laboratoire d'histocompatibilité de Genève qui gère, dans la plupart des cas, la répartition des organes ainsi disponibles ${ }^{\mathbf{2 6 1}}$.

Dans le cas de la transplantation de cœur et de foie, l'officialisation d'une collaboration entre les médecins des différents centres par l'édiction de règles d'échange ne fait pas d'emblée l'unanimité. Les chirurgiens transplanteurs hépatiques reconnaissent la pertinence de se rencontrer pour discuter des questions organisationnelles et scientifiques liées à la réalisation de transplantation de foie, toutefois l'élaboration de statuts officiels et de règles fixes ne leur paraît pas impérative. C'est ce que rapporte François Mosimann, chirurgien au CHUV, lors d'une réunion du Conseil de fondation:

"À l'heure actuelle, chaque centre transmet sa propre liste d'attente de receveurs potentiels. Lorsqu'un donneur est disponible, les arrangements pour le prélèvement se font par contact direct avec les centres. Ce mode de faire ayant l'air de convenir pour l'instant à la majorité, le projet de statuts reste encore en veilleuse ${ }^{262}$.»

Dans le cas de la transplantation cardiaque, aucune règle n'est établie, mais le groupe ad hoc opte pour la diffusion des listes d'attente de patients entre les centres afin de permettre aux médecins de tenir compte du critère d'urgence dans la répartition des organes ${ }^{\mathbf{2 6 3}}$. Ce partage de données représente ainsi un premier pas vers une

261 Correspondance, "Poste de secrétaire pour l'organisation Swisstransplant à Genève", 29 mars 1990 , p. 1.

262 P-V CF, 22 juin 1988, p. 3.

263 P-V CF, 2 décembre 1987, p. 3. 
recherche de formalisation des règles d'échanges, bien qu'il soit difficile d'évaluer son influence sur les usages concrets et sa prise en compte par les acteurs concernés.

\section{Réguler pour optimiser les dons}

L'introduction progressive de règles d'échange au début des années 1990 vise à normaliser la collaboration entre les centres, notamment dans le cas d'organes non utilisables localement. Avec la création de la Coordination nationale, le modèle de répartition entre centres passe en effet de l'échange ponctuel des organes sur une base parfois affinitaire ${ }^{264}$ à un partage des organes fondé uniquement sur des règles médicales que les groupes de travail par organe, constitués par des représentants de chaque centre, établiront. Il s'agit pour les centres de transplantation de suivre des normes formelles définissant précisément les usages.

D'un côté, la suppression des arrangements informels entre transplanteurs permet à Swisstransplant de rendre le processus de répartition des organes transparent et d'en assumer publiquement la gestion afin d'éviter l'intervention d'acteurs extérieurs. De l'autre, ce changement marque également la volonté d'optimiser l'utilisation des organes disponibles. Dans la demande de financement transmise à la CDS en 1991, l'argumentaire aborde sans détour cet aspect, comme l'illustre cet extrait :

«Pour un échange efficace d'organes au plan suisse utilisant au mieux le potentiel des donneurs de ce pays, il est absolument nécessaire de disposer d'un service de coordination à l'échelon national ${ }^{265}$."

Si cette structure revêt une importance organisationnelle, elle offre surtout la garantie aux centres de pouvoir bénéficier d'organes. La réalisation des transplantations pour un hôpital ne dépend donc plus exclusivement des prélèvements qu'il réalise in situ. Pour permettre le fonctionnement de ce système de gestion de la transplantation, le principe de base réside dans l'annonce systématique du donneur à

264 Comme cela a été relaté lors des entretiens menés avec les médecins.

265 Demande adressée par la Fondation Swisstransplant à la Conférence des directeurs cantonaux des affaires sanitaires, mars 1991, p. 15. 
la Coordination. Préalable à tout prélèvement, cette annonce doit être faite par chaque hôpital dans lequel un donneur est identifié. Si la mise à disposition d'organes dépend alors de la communication effectuée par chaque centre, le personnel de la Coordination est amené à jouer un rôle actif dans ce processus en vérifiant lors de l'annonce avec le médecin que tous les organes potentiellement utilisables d'un donneur ont bien été prélevés. Lors de l'identification d'un donneur, certains organes comme les poumons ne semblent pas, en effet, faire systématiquement l'objet d'une évaluation. Ainsi, dans le cadre des directives concernant la transplantation de cœur et cœur-poumon, une mention rappelle que même lors de prélèvement de cœur, "vu la rareté des greffons pulmonaires sains, à chaque occasion qu'un donneur se présente, il est vivement recommandé de s'enquérir si les poumons sont utilisables ${ }^{\mathbf{2 6 6}}$ ».

Une fois l'annonce transmise, la Coordination attribue l'organe à un hôpital selon des critères d'échange établis par les groupes de travail en fonction des particularités de chaque organe. La répartition d'organes s'effectue ainsi selon des normes médicales et organisationnelles formulées par les transplanteurs eux-mêmes. Dès 1992, les groupes de travail sont invités par Swisstransplant à élaborer ou réévaluer les normes qui régulent la distribution des ressources qui les concernent. Entérinée par le Comité exécutif de Swisstransplant, l'existence de règles de répartition des organes entre centres devient une exigence pour intégrer la fondation. Au début de la mise sur pied de la centralisation des échanges, certains groupes de travail, comme celui pour le cœur, mettent plus de temps que d'autres à établir des règles d'échange. Ainsi, dans le cas de la distribution des cœurs, sans règles d'échange écrites fournies par le groupe de travail, la Coordination limite provisoirement son rôle, lorsqu'un donneur est annoncé, à proposer le cœur aux trois hôpitaux, qui s'accordent ensuite entre eux ${ }^{267}$. La mise par écrit d'usages encore souvent informels contribue à les doter d'un caractère institutionna-

266 Correspondance, "Règles de répartition des greffes de cœur, cœur-poumons et poumons en Suisse", 23 juin 1992, p. 1.

267 Correspondance, "Règles d'échanges des cœurs au niveau de la Coordination nationale", 14 septembre 1992. 
lisé. La coordination vise surtout à clarifier la pratique et pas uniquement à la réguler. Ainsi le groupe de travail de transplantation hépatique décide de conserver la règle en vigueur. La répartition est effectuée selon une ordination alphabétique des établissements, le centre bénéficiant de l'organe étant relégué en dernière position lors de l'appel suivant de manière à organiser un tournus ${ }^{268}$.

La liberté scientifique laissée aux groupes de travail - constitués par les transplanteurs et médecins en activité, répartis par organe dans les différents centres de transplantation par le Comité exécutif est significative. Les acteurs impliqués au plus proche de la pratique, les chirurgiens-transplanteurs au premier chef, proposent les règles quelles que soient les conditions de leur élaboration. Dans le cas de la transplantation de pancréas menée dans les années 1990 dans deux hôpitaux, cette élaboration prend la forme d'un échange épistolaire entre Philippe Morel et Felix Largiadèr, responsables respectivement des programmes à Genève et à Zurich :

«Comme nous en avions convenu, je me permets de te faire une première proposition pour établir une règle d'échange des greffons pancréatiques, accompagnés ou non d'une greffe rénale. [...] Cette proposition est un point de départ pour établir une règle, et j’attends bien sûr tes propositions et ton avis ${ }^{269}$."

Si les groupes ont donc le champ libre pour élaborer les règles qui conviennent le mieux aux spécificités de l'organe dont ils se chargent, il arrive que Swisstransplant les établisse lui-même, par le biais du Comité exécutif et de son président Morel. C'est le cas de la transplantation cardiaque pour laquelle les représentants des établissements hospitaliers ne parviennent pas à se mettre d'accord:

«Je souligne que cette règle est temporaire, et qu'elle prendra

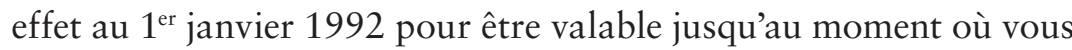
aurez mis en place, dans le cadre du groupe STAH [Swisstransplant 
Arbeitsgruppe Herz], une autre règle d'échange qui paraîtra opportune ${ }^{270}$.»

Toutefois, l'importance du statut provisoire et modifiable de ces règles est largement soulignée. Une fois un accord trouvé entre les différents membres du groupe de travail, elles gardent un caractère modulable dont il est souvent fait mention. Le but recherché par l'édiction de règles n'est en aucun cas d'entraver la pratique des transplantations dans les centres. La responsabilité de l'élaboration laissée aux groupes et donc aux transplanteurs eux-mêmes est un signal fort informant sur l'importance de la prise en compte de la réalité pratique.

Les normes revêtent une forme spécifique selon l'organe, mais également en fonction des problèmes organisationnels ou logistiques rencontrés dans la pratique. Le développement de nouveaux types de transplantations entraîne parfois l'ajustement de règles déjà en vigueur. C'est le cas pour les interventions couplées pancréas-rein: lorsqu'un hôpital réalise l'opération après avoir reçu à la fois un pancréas et un rein, il est, selon les règles établies pour la transplantation rénale, tenu de mettre à disposition des autres centres le prochain rein prélevé. Dans ce cas précis, cette règle menace le programme de transplantation rénale de l'établissement en le rendant redevable d'un organe qui n'a pas été distribué à un patient en attente d'un rein, mais d'un pancréas. Ce type de problème pratique nécessite une redéfinition des usages que le groupe de travail est chargé d'accomplir.

L'importance du Comité exécutif ne se limite donc pas à permettre aux centres de proposer un représentant, mais constitue un espace de travail concret sur la transplantation. À cet égard, un établissement recommande un interniste comme représentant, ce qui suscite la réprobation du président du Comité exécutif qui se demande «comment un interniste peut être un interlocuteur valable dans les décisions que nous devons prendre ${ }^{271}$ ». Garantie

270 Correspondance, "Règles d'échanges cardiaques", 30 décembre 1992.

271 Correspondance, "Représentation de Lausanne au sein du Conseil exécutif de Swisstransplant", 9 juillet 1992. 
de l'expertise des activités scientifiques qui y sont effectuées, l'usage veut que seuls des chirurgiens soient nommés comme représentants des centres au Comité exécutif ${ }^{272}$.

Après 1992 et la mise en place de la Coordination nationale, la diversification des types de transplantations et l'introduction de nouveaux programmes impliquent la création d'un groupe de travail reconnu par Swisstransplant. C'est le cas du groupe cœurpoumon qui, constitué informellement, demande son intégration à la fondation:

"Nous souhaiterions, au plan suisse, coordonner les efforts de développement dans le domaine de la transplantation pulmonaire et cardio-pulmonaire, qui recouvre une pathologie distincte de celle justifiant une transplantation cardiaque seule ${ }^{273}$.»

L'avis des membres du Comité exécutif est recueilli par le président avant que celui-ci ne réagisse officiellement:

"J'ai envoyé ce jour une lettre aux membres du comité exécutif leur demandant leur avis quant à votre proposition qui, personnellement, me paraît particulièrement opportune et judicieuse. Je pense que ce groupe pourrait être constitué de la même façon que les autres groupes déjà existants ce qui ne nécessiterait aucune procédure particulière si ce n'est l'organisation interne de ce groupe avec la désignation d'un Président renouvelé annuellement ${ }^{274}$.»

La reconnaissance de la légitimité médicale et organisationnelle des groupes de travail constitués par organe vise à garantir l'intégration au système de coordination nationale. S'il est théoriquement possible qu'un centre de transplantation décide de ne pas intégrer le système d'échange, une telle décision impliquerait la totale autonomie et l'isolement complet des médecins de l'hôpital. En plus d'empêcher tout échange d'organes, désormais régulé par la Coordination, le recours aux infrastructures techniques de la fondation, comme le laboratoire d'histocompatibilité, serait alors refusé. La configuration

272 Correspondance, "Représentation du CHUV au comité exécutif de Swisstransplant", 29 octobre 1998.

273 Correspondance, sans titre [demande de création d'un groupe pour la transplantation pulmonaire et cardio-pulmonaire], 8 septembre 1992.

274 Correspondance, "Votre courrier du 8.9.92", 28 septembre 1992. 
de la gestion établie par Swisstransplant rend donc difficile le développement de programmes de transplantation hors de son contrôle.

\section{Dans la pratique}

L'application des règles et le partage d'organes par la Coordination ne se font pas toujours sans difficulté. Comme expliqué plus haut, lorsque le prélèvement est effectué dans un hôpital universitaire, son centre de transplantation est prioritaire avant que l'organe, le cas échéant, ne soit proposé à d'autres centres. La répartition des organes se complexifie lorsque le prélèvement est réalisé dans un hôpital périphérique sans programme de transplantation. La résistance aux usages d'échanges, tels que définis par la Coordination, y est parfois plus marquée. C'est le président du Comité exécutif qui se charge, par de nombreux courriers, de veiller au respect des règles nouvellement codifiées. Il arrive qu'un hôpital périphérique souhaite, par exemple, que l'organe prélevé soit transmis à un centre en particulier ${ }^{275}$, comme le relate Morel, après enquête:

«Concernant le donneur qui a été prélevé à Sion par notre équipe [...], je vous informe que l'Hôpital de Sion a fait appel au Centre de Genève et non pas à la Coordination nationale comme cela m'a été clairement confirmé pour contrôler ce point-làa ${ }^{276}$.»

C'est pour éviter la persistance des usages informels que Swisstransplant veille fermement au respect des règles de fonctionnement de la coordination. Lorsque des établissements procèdent à des prélèvements sans les annoncer à la Coordination et que l'infraction est découverte, l'envoi d'un courrier par le président du Comité est automatique. Swisstransplant exerce par ce biais une pression sur les hôpitaux en leur rappelant les règles de fonctionnement:

«La Coordination nationale ne peut fonctionner si elle n'est que partiellement au courant des activités de transplantation exercées dans notre pays ${ }^{277}$.»

275 Correspondance, "Règles d'échanges concernant les foies", 9 novembre 1992.

276 Correspondance, "Votre lettre du 5 octobre 1992", 13 octobre 1992.

277 Correspondance, "Donneur d'organes (cœur et reins) prélevé dans votre centre le 7.9.1992", 28 septembre 1992. 
Si les arrangements et liens d'affinité doivent être exclus pour permettre à la coordination nationale de fonctionner, c'est également pour lever toute opacité sur les pratiques et l'origine des organes. Cet aspect est surtout mis en exergue lorsque des transplantations sont réalisées grâce à des organes provenant de l'étranger. C'est ce qu'évoque un courrier transmis à l'hôpital de Zurich, où une transplantation est effectuée par ce biais sans avoir averti la Coordination nationale:

«Il est vrai que par le passé des importations directes d'organes ont été faites de centres étrangers vers la Suisse, tu conviendras certainement avec moi qu'actuellement le passage par notre organisme national est impératif si l'on veut présenter à nos autorités politiques et à nos concitoyens toutes les garanties indispensables pour prévenir toute tentative illicite d'organes dans le Cercle de la Transplantation Suisse ${ }^{278}$. »

En plus de remettre en cause le principe de transparence, l'intervention effectuée à l'insu de la Coordination entraîne une répartition inéquitable des organes entre les centres suisses. En omettant l'annonce de ce don, l'établissement zurichois garde la place qu'il occupe dans la liste d'attribution au lieu d'être relégué en dernière position, comme le veut la règle élaborée par le groupe de travail.

Le système de coordination nécessite la participation de l'ensemble des institutions hospitalières pour fonctionner efficacement. Si les centres de transplantation y trouvent l'avantage de ne plus dépendre uniquement de leurs propres prélèvements pour mener des transplantations, l'implication des établissements périphériques est moins évidente. L'identification et la prise en charge d'un donneur cadavérique dans l'attente du prélèvement engendrent des coûts et détournent le personnel soignant des tâches habituelles. Lorsque la fondation est avertie de problèmes dans l'organisation de prélèvements survenus dans ces établissements, elle prend un soin particulier à les ménager pour s'assurer de leur collaboration. L'épisode suivant intervenu en 1994 en donne l'illustration. Après l'annonce d'un donneur identifié dans un hôpital périphérique dépourvu de

278 Correspondance, "Transplantation effectuée dans le centre de Zurich par un donneur provenant de Birmingham-UK», 4 mai 1994, p. 2. 
centre de transplantation, l'équipe responsable du prélèvement se rend sur place avec un retard considérable. Afin d'éviter le dommage irrémédiable des organes, un chirurgien local, au bénéfice d'une expérience dans le domaine de la transplantation acquise au cours de sa carrière, avait procédé lui-même à la préparation du donneur. Devant cette situation contraignante pour l'établissement dont le fonctionnement habituel est bouleversé ainsi que pour les activités du chirurgien et du personnel soignant, Morel se charge de transmettre un courrier personnel au médecin qui a informé la Coordination de la présence d'un donneur dans lequel il exprime sa vive reconnaissance à l'effort consenti :

"Je voudrais d'abord vous remercier très sincèrement de la contribution si importante que vous avez consentie en nous donnant la possibilité d'effectuer dans votre Hôpital un prélèvement d'organes. Pour avoir travaillé moi-même en dehors des centres universitaires, je sais parfaitement quelle est la charge que représente une telle situation. Afin de s'assurer de votre collaboration, il est bien entendu nécessaire que nous mettions à votre disposition une organisation qui soit dans la mesure du possible irréprochable ${ }^{279}$.»

La crainte de voir les hôpitaux périphériques perdre la motivation à identifier des donneurs potentiels est évoquée par Morel dans un courrier qu'il transmet au centre concerné par cette affaire:

"[...] Je me permets de vous demander s'il était possible de faire en sorte que de telles situations se reproduisent aussi rarement que possible, car elles entraînent pour les hôpitaux donneurs, surtout lorsqu'il s'agit d'hôpitaux régionaux, des conséquences extrêmement fâcheuses et peuvent même parfois conduire à la perte du donneur en question, et d'autres donneurs potentiels ultérieurs ${ }^{\mathbf{2 8 0}}$.»

On constate l'ascendant que prend Swisstransplant dans la régulation des pratiques médicales effectuées par les centres de transplantation, laquelle exige une uniformisation et une standardisation que les situations particulières doivent perturber le moins possible.

279 Correspondance, "Votre courrier du 15 février 1994 ", p. 1.

280 Correspondance, "Concerne donneur annoncé à votre centre le 15 février 1994 ", 18 février 1994. 
L'équilibre fragile entre les différents acteurs de la chaîne doit faire l'objet de précautions pour ne pas remettre son fonctionnement en cause.

\section{Remise en cause du modèle d'attribution}

Si la répartition des organes entre les centres est effectuée par le biais de la Coordination nationale selon des règles établies, les critères d'attribution aux patients, c'est-à-dire à l'intérieur des centres, sont moins clairs et ne font l'objet d'aucune coordination de la part de Swisstransplant.

Issus d'un développement initial de la transplantation sous la seule impulsion des hôpitaux, ces critères sont fixés par les transplanteurs leur assurant une grande liberté. L'adaptation aux situations médicales et logistiques particulières en représente le principal avantage. Une maladie passagère contractée au dernier moment par le receveur peut, par exemple, remettre en cause une intervention, même si tous les critères médicaux préalables sont réunis. Grâce à la marge de manœuvre dont bénéficient les médecins, l'organe sera dans la mesure du possible attribué à un autre patient du centre ${ }^{281}$.

Dans le cadre du processus d'élaboration de la loi sur la transplantation en 2000, ce système d'attribution est contesté par des acteurs extérieurs au domaine de la transplantation. La préférence de la Confédération pour un modèle dans lequel les centres de transplantation ne bénéficient d'aucune prérogative sur les organes prélevés est en effet pressentie dès la fin des années 1990. Dans l'attente d'une prise de position au grand jour des autorités fédérales, le Comité exécutif défend le modèle en vigueur. Lors d'une rencontre avec les représentants de l'OFSP en 1998, les membres du Comité s'efforcent de prouver que l'attribution des organes est garantie par une supervision d'experts médicaux à tous les stades du processus, dans les centres et les groupes de travail:

«Swisstransplant est CONTRE l'idée d'une attribution centrale et nominative des organes et POUR une attribution au centre de

281 Correspondance, « Anfrage von Herrn Professor Zeltern, BAG, vom 13. November 2000 betreffend Organisation und Qualität der Transplantationszentren », 25 juin 2001. 
transplantation pour lui choisir le patient le plus approprié. Le système actuel donne une chance égale à tous les patients à cause de la flexibilité, rapidité et transparence actuelle. [...] La prérogative et le droit de regard des centres de transplantation sont respectés, les avis des groupes de travail sont pris en compte et la communication journalière entre les médecins permet une grande flexibilité et surtout des interventions rapides si c'est nécessaire ${ }^{282}$."

En novembre 2000, un courrier de l'OFSP adressé à l'ensemble des centres de transplantation aborde formellement la question de l'attribution. Sans toutefois constituer une remise en cause du modèle en vigueur, il cherche à clarifier les critères qui justifient la distribution d'un organe ainsi que l'inscription d'un patient sur la liste d'attente de chaque centre ${ }^{283}$. Perçue comme une menace pour le système en vigueur, cette sollicitation fait l'objet d'une prise en main par le Comité exécutif. Dans l'optique de clarifier les pratiques hospitalières, il demande en effet à chaque établissement transplanteur de transmettre l'ensemble de leurs critères d'attribution des organes et d'inscription des patients sur les listes d'attente. La compilation des usages internes par le Comité contribue à officialiser des pratiques internes alors peu formalisées. La fondation démontre qu'audelà des règles d'échange, c'est l'ensemble du processus d'attribution des organes aux patients qui est coordonnér84.

L'explicitation des mécanismes d'attribution dans les courriers envoyés par les centres vise à convaincre que les médecins appliquent les critères médicaux. Christian Seiler, au nom de la clinique de chirurgie viscérale et de transplantation de Berne, explique dans sa réponse que ces critères sont élaborés et évalués à trois niveaux:

"Ces critères ont été, d'un côté, définis dans le cadre de conférences internationales, d'un autre les changements de détails ont été discutés au sein du STAL [Swisstransplant Arbeitsgruppe Leber] et par les équipes locales de transplantation, sous la direction de

282 P-V CE et OFSP, 12 mai 1999, p. 3.

283 Correspondance, "Attribution des organes et qualité des centres de transplantation", 13 novembre 2000.

284 P-V CM, 4 décembre 2000, p. 2. 
chirurgiens, hépatologues, immunologues, gastroentérologues et anesthésistes ${ }^{285}$.»

De la même manière, plusieurs médecins insistent sur la participation de nombreux spécialistes issus de différentes disciplines médicales dans le processus d'attribution des organes. Pour la transplantation rénale, Jean-Pierre Wauters du CHUV explique sobrement que «toutes les décisions habituellement prises par une équipe de transplantation regroupent des néphrologues et chirurgiens ${ }^{\mathbf{2 8 6}}$ ». Garants de la prise en compte de la diversité des facteurs médicaux en jeu, la pluralité de médecins spécialistes exclut une décision arbitraire, le transplanteur n'étant qu'un maillon d'un ensemble plus vaste. De fait, son poids dans le processus d'attribution est identique à celui de ses collègues. Dans cette optique, selon le chirurgien du CHUV, il serait même dangereux de confier une tâche nécessitant la considération de nombreux aspects médicaux à un seul individu. Si des discussions entre spécialistes sont nécessaires, c'est en raison de la complexité des aspects médicaux en jeu. Dès lors, à ses yeux, un système centralisé indépendant des unités de transplantation représente un non-sens médical et scientifique. L'importance des critères médicaux n'exclut pas la prise en compte d'aspects complémentaires, à l'instar du temps d'attente après inscription sur la liste. Dans la réponse que transmet la clinique de chirurgie viscérale et de transplantation de Berne, par exemple, le temps d'attente après inscription sur la liste constitue l'un des éléments pris en considération:

«Il s'agit de critères purement médicaux, basés sur des directives internationales, dans lesquels le temps passé sur la liste a aussi été pris en considération ${ }^{287}$.»

Si le processus de clarification des critères d'attribution dans les centres à la demande du Comité exécutif a pour objectif d'homo-

285 Correspondance, "Anfrage von Herrn Professor Zeltern, BAG, vom 13. November 2000 betreffend Organisation und Qualität der Transplantationszentren", 25 juin 2001, p. 2 [notre traduction].

286 Correspondance, "Description des critères de mise en liste et d'allocation d'organes concernant la transplantation rénale", 21 septembre 2001.

287 Correspondance, "Anfrage von Herrn Professor Zeltern, BAG, vom 13. November 2000 betreffend Organisation und Qualität der Transplantationszentren ", 25 juin 2001 [notre traduction]. 
généiser formellement les pratiques, on constate peu de différences dans la réalité clinique entre les centres. Les critères d'attribution des organes et d'inscription des patients sont élaborés par le groupe de travail avant d'être discutés et évalués par les médecins des centres, alors que l'hôpital, en tant qu'institution, ne semble pas avoir un poids décisif. Basée sur des critères médicaux définis et reconnus par des experts internationaux, l'attribution laisse peu de place à l'arbitraire ou à l'interprétation personnelle. Dans cette optique, il semble que les critiques émises par l'OFSP contre ce modèle ciblent plutôt l'implication prépondérante des centres dans le processus d'échange que les usages, même non écrits, qui potentiellement nourrissent des idées d'attribution aléatoire ${ }^{288}$.

Si les explications fournies par les médecins mettent en avant les critères médicaux et scientifiques dans l'attribution des organes, elles soulignent toutefois la prééminence des intérêts de chaque centre de transplantation dans l'ensemble du processus. La priorité dont bénéficie le centre responsable du prélèvement contribue à lier l'organe au centre par un droit de propriété. C'est l'existence de ce droit qui est contestée ${ }^{289}$. D'une ressource appartenant aux hôpitaux, l'organe doit acquérir le statut de bien attribuable à n'importe quel patient selon des critères qui garantissent un partage équitable. Le modèle alternatif évoqué par l'OFSP qui propose d'axer l'attribution des organes en fonction d'une liste d'attente nationale unique doit permettre cette transformation conceptuelle. En effet, le principal grief des autorités fédérales réside dans l'injustice qu'entraîne du point de vue des patients la répartition fondée sur la priorité accordée au centre où le prélèvement est effectué290. Dans un des projets de loi élaborés en 2001, l'OFSP transmet les critères qui, selon l'avis des instances fédérales, doivent garantir une attribution équitable:

«Lors d'allocation d'organes, l'objectif principal est l'équité. [...] Pour atteindre cet objectif, la loi soutient le principe que personne

288 Correspondance, "Règles de mise en attente des patients et règles d'attribution des organes", 7 mai 2002.

289 Correspondance, sans titre [avis de l'OFSP sur les critères de mise en liste d'attente et d'allocation d'organes], 15 février 2002, p. 2-3.

290 Pour les aspects éthiques de cette question, voir Bondolfi 2002. 
ne doit subir de discrimination dans la répartition d'un organe. Comme critères déterminants ne doivent entrer en considération que l'urgence médicale, les bénéfices médicaux d'une transplantation et le temps d'attente ${ }^{291}$.»

Pour l'OFSP, les critères médicaux ne représentent pas une fin en soi, mais sont des indicateurs de l'urgence et la nécessité. Ce modèle permet également d'annuler l'inégalité induite par l'attribution des organes selon un système de rotation, où les centres reçoivent chacun à leur tour un organe. Les patients inscrits sur les listes d'attente des centres de taille importante, comme Zurich où de nombreux patients sont inscrits, sont défavorisés. Ce constat pousse d'ailleurs les chirurgiens Walter Weder et Nicolas Demartines du département de chirurgie de l'hôpital de Zurich à expliciter à la présidente Trix Heberlein le problème qui les concerne particulièrement:

«Les listes d'attente dans les différents centres sont de grandeur variable, ce qui signifie que le système est inégal pour les patients. Dans les grands centres où se trouvent des longues listes d'attente (c'est le cas par exemple de la transplantation de foie), les patients sont largement désavantagés par rapport à ceux qui sont inscrits dans des centres avec une liste courte ${ }^{292}$.»

Le problème réside moins dans les critères d'attribution ou de mise sur liste d'attente au sein des centres que dans les règles d'échange appliquées par la Coordination nationale. À l'origine de cette structure s'exprime la volonté du corps médical d'établir une utilisation optimale des organes disponibles. La répartition équitable entre les patients n'est pas un aspect a priori prépondérant. Il s'agit surtout de garantir, selon des règles d'échange, la circulation des organes, lorsque la transplantation locale n'est pas envisageable. Dans ce cas seulement, l'organe est proposé à d'autres établissements selon les règles édictées par chaque groupe de travail. Cette priorité

291 Botschaft zum Bundesgesetz über die Transplantation von Organen, Geweben und Zellen (Transplantationsgesetz), 2001 [notre traduction].

292 Correspondance, sans titre [département de chirurgie de l'hôpital universitaire de Zurich à propos du projet de loi transmis à soumission des centres de transplantation], 15 février 2002, p. 1 [notre traduction]. 
contribue à lier, au sein des centres, le processus du don à ceux du prélèvement et de la transplantation.

Héritée de la période d'émergence de la transplantation, la priorité des centres chargés du prélèvement sur l'organe représente une forme d'adaptation des pratiques cliniques à un contexte technique où la maîtrise de la conservation des organes est encore peu développée. Cette réalité technique impose une utilisation rapide des greffons et rend l'établissement d'échanges à grande échelle difficile. Leur réalisation dépend largement des prélèvements effectués in situ donnant ainsi aux centres un rôle majeur dans l'ensemble du processus de transplantation. L'expérience technique, médicale et organisationnelle acquise dans les hôpitaux depuis la mise en place des programmes à la fin des années 1980 entraîne sans doute une gestion plus efficace par le personnel médical de la procédure du don d'organes. Aussi, la marge de manœuvre laissée jusque-là aux hôpitaux est moins nécessaire, rendant le passage à un modèle d'attribution centré sur les patients envisageable. Si la possibilité de remise en cause du système de répartition forgé entre médecins est sans doute issue d'un changement conceptuel dans la perception de l'organe ou des besoins des patients, l'évolution de la maîtrise des techniques rend ce projet médicalement réalisable.

Bien que Swisstransplant entende défendre le modèle de répartition établi au début des années 1990, le changement semble déjà irrévocable au début des années 2000. Reste pour la fondation à négocier le rôle qu'elle endossera dans ce nouveau système, comme le laissent penser les propos relatés lors d'une séance du Conseil de fondation: "Avec le texte de loi, on peut vivre. Les problèmes sont la compétence à la mettre en place ${ }^{293}$.»

\subsection{Garantir la compétence des centres de transplantation}

Pour rappel, lorsque Swisstransplant est créée en 1985, la configuration hospitalière de la transplantation est fixée, sans pour autant avoir fait l'objet d'un plan de développement politique ou 
médical établi. Les transplantations sont uniquement effectuées, au même titre que d'autres interventions lourdes, dans les hôpitaux au bénéfice de ressources logistiques suffisantes. Cette configuration issue d'un pragmatisme matériel est tacitement acceptée en raison des spécificités techniques de pointe que la transplantation requiert. Vers la fin des années 1980, la normalisation des pratiques médicales tend à rendre la réalisation de certains types de transplantations plus commune. Se pose donc la question du partage des compétences et de l'habilitation à mener ce type d'intervention. En 1988, lors d'une séance du Conseil de fondation, Bruno Speck évoque à propos des transplantations de moelle allogénique la nécessité de contrôler le développement des centres:

«Selon les critères internationaux, seuls les hôpitaux pratiquant plus de vingt transplantations par an devraient avoir une reconnaissance officielle. En Suisse, un seul centre, celui de Bâle, atteint un tel chiffre; il serait dès lors totalement aberrant d'ouvrir d'autres centres $^{294}$.»

Swisstransplant recommande alors de limiter la transplantation aux centres en activité. La fondation, constituée de transplanteurs de chaque établissement, n'a pas pour objectif de diminuer le nombre de centres. Toutefois, la crainte de voir la transplantation discréditée par des pratiques qui échapperaient à la réglementation renforce la volonté d'une régulation. Derrière cette restriction, il s'agit également pour la fondation de donner une reconnaissance au système de gestion de la transplantation qu'elle a établi.

\section{Les cliniques privées}

Les réflexions sur la limitation du nombre de centres émergent principalement en réaction à l'activité de transplantation dans des cliniques privées. Une, au moins, propose des transplantations rénales et cardiaques dans le canton de Vaud à la fin des années $1980^{295}$. Dans un courrier transmis par Andreas Graf, chirurgien exerçant en milieu privé, dans lequel il donne son avis sur une action d'infor-

294 P-V CF, 22 juin 1988, p. 6.

295 Idem, p. 2. 
mation du public développée par la fondation, il fait clairement référence à l'expérience qu'il y a acquise dans ce domaine:

«Je le fais en qualité de chirurgien cardiaque, confronté directement avec le problème de la transplantation puisque j'ai effectué à ce jour six transplantations cardiaques en milieu médical privé romand ${ }^{296}$.»

La principale préoccupation des membres de la fondation est le flou qui entoure la provenance des organes transplantés en milieu privé. Une clinique qui ne possède pas d'unité de soins intensifs où un donneur pourrait être identifié dépend exclusivement d'établissements publics pour offrir ce type de prestations médicales à ses clients. Les réunions du Conseil de fondation laissent entendre que les organes utilisés par ces cliniques ne peuvent, selon l'avis des membres, provenir en aucun cas d'hôpitaux publics. Les échanges d'organes, s'ils ne sont pas encore coordonnés à la fin des années 1980 par Swisstransplant, ne se réalisent qu'après un accord entre l'hôpital où le prélèvement est effectué et celui auquel l'organe est cédé. Des recherches sont menées sur les conditions d'approvisionnement de la clinique concernée. Un des membres du Conseil de fondation parvient à établir que les organes sont cédés par un hôpital étranger en suivant un circuit parallèle à ceux que les institutions en Europe et en Suisse tentent de mettre en place. Les organes sont mis à disposition de la clinique par «un arrangement avec le centre de Barcelone qui semble avoir un excédent de cœurs et qui ne veut pas les offrir à France Transplant, pour des raisons non explicitées ${ }^{297}$ ».

On constate donc que la pratique des transplantations dans la clinique vaudoise s'appuie sur la mobilisation de moyens que la mise en place de règles d'échange a pour objectif de faire disparaître. Issu d'accords soustraits à toute réglementation, ce démarchage parallèle remet en cause le bien-fondé des actions entreprises basées sur la coordination et la transparence, mais court-circuite également les critères de répartition.

296 Correspondance, "Don d'organes, votre lettre du 19 janvier 1989 ", 30 janvier 1989, p. 1. 297 Note du 2 août 1990 pour dossier du Comité exécutif de la fondation Swisstransplant, p. 1. 
La commercialisation de ce type d'opérations en milieu privé est également un problème que les membres de la fondation perçoivent comme potentiellement menaçant pour la poursuite des transplantations dans les établissements publics. La crainte est de voir les critères médicaux définissant un receveur idéal remplacés par les moyens financiers du patient. Un courrier rédigé par un membre à l'attention du Comité exécutif le met particulièrement en exergue:

«La transplantation exige une transparence absolue, en partie en ce qui concerne la provenance des donneurs, et que d'autre part les règles éthiques doivent être scrupuleusement respectées en particulier celles qui veulent qu'un patient ne doive pas pouvoir bénéficier en priorité d'une transplantation cardiaque parce qu'il a les moyens de payer des soins dans une clinique privée ${ }^{298}$."

Le problème prend une ampleur inédite avec la mise en place de la Coordination nationale. La transplantation est désormais une pratique médicale régie par des règles logistiques dont Swisstransplant est garante. Façonnées par les acteurs directement concernés et validées par Swisstransplant, ces normes consacrent l'expertise de la fondation à administrer l'organisation de la transplantation. Les enjeux s'articulent donc moins autour de la reconnaissance de la capacité médicale des cliniques privées à effectuer ce type d'opérations que de la légitimité à gérer et à contrôler leur réalisation. Dès lors, la fondation décide d'informer la CDS de ces pratiques en l'invitant à limiter officiellement la transplantation aux six centres en activité.

La réalisation de transplantations dans les cliniques privées n'est pas directement mentionnée, mais l'argumentation développée vise à rendre l'activité de transplantation dans ces établissements incompatible avec des critères de qualité, définis par la fondation. Sans se limiter uniquement à prendre les cliniques pour cibles, la demande met en exergue la nécessité de respecter des indications médicales et éthiques afin de permettre une attribution équitable des organes pour chaque patient. Les propos formulés dans l'introduction du courrier s'efforcent de démontrer que Swisstransplant et les centres de transplantation constituent l'unique structure garantissant cet objectif: 
«La fondation Swisstransplant dans laquelle les six centres de transplantation sont représentés a pour objectif de permettre à chaque patient dans notre pays qui, en raison de critères médicaux stricts, nécessite une transplantation d'organes de subir dans un délai utile une opération de transplantation ${ }^{299}$. »

Les critères scientifiques et médicaux consistent à faire des hôpitaux universitaires les seuls établissements à l'infrastructure suffisamment développée pour y mener des transplantations ${ }^{300}$. La disponibilité d'une équipe chirurgicale techniquement entraînée et prête à intervenir constamment représente un des impératifs que les cliniques privées ne peuvent assurer. Peu compatible avec l'offre thérapeutique médicale segmentée des cliniques, la disponibilité de spécialistes variés est également jugée indispensable en cas de complications soudaines. Le suivi postopératoire, basé sur une connaissance précise des dosages médicamenteux immunosuppressifs, est présenté également comme décisif pour le succès d'une transplantation. La surveillance à long terme des patients transplantés pose la question de la possibilité de ce type de prise en charge dans les établissements privés.

L'influence des aspects financiers dans l'indication à la réalisation d'une transplantation est également évoquée ${ }^{301}$. Dans les centres, les transplantations suivent des critères médicaux qui font l'objet de réévaluation par des groupes d'experts. L'interdisciplinarité des équipes mobilisées pour prendre la décision de pratiquer la transplantation est présentée comme un garde-fou contre une trop grande tendance à opérer. L'ensemble du corps médical ne tire aucun avantage financier à pratiquer une transplantation, alors que dans une clinique elle vise la rentabilité. Dans cette optique, le risque d'un recours hâtif à la transplantation, au détriment d'autres types d'opérations moins lucratives, dans le but d'augmenter les profits des cliniques, en est la principale conséquence.

299 Correspondance, "Anforderung an die zur Durchführung von Organtransplantationen berechtigten Spitäler", 20 janvier 1992, p. 1 [notre traduction].

300 Idem, p. 4.

301 Idem, p. 3. 
Enfin, la réalisation de transplantations au sein de cliniques privées est également éthiquement contestable pour la fondation. Dans un contexte de pénurie, la concurrence pour l'acquisition d'organes créée par l'émergence de cliniques est mal perçue ${ }^{302}$. Les établissements privés, sans lien d'appartenance à un réseau d'échanges, sont libres d'utiliser des organes dont la provenance est souvent incertaine, favorisant la persistance d'un marché parallèle. Dans les centres de transplantation des hôpitaux universitaires, la provenance des organes est assurée puisque chaque prélèvement effectué fait l'objet d'une annonce à la Coordination.

Si la CDS reconnaît la nécessité de réfléchir à une régulation de ce type d'activité, elle ne peut formellement circonscrire une activité médicale à certains établissements seulement comme l'illustre le passage de cette lettre, signée par son président le conseiller d'État saint-gallois, transmise au président de la fondation en septembre 1992:

"L'introduction d'une obligation de requérir une autorisation spéciale pour effectuer des transplantations ne va pas sans poser des problèmes juridiques, car l'autorisation délivrée aux médecins couvre en principe tous les actes médicaux. Encore moins pour des motifs de droit constitutionnel, on ne peut interdire à des médecins travaillant dans un établissement privé de procéder à des actes admis par la loi. Il est donc exclu que les institutions privées soient défavorisées par rapport aux hôpitaux publics en ce qui concerne la légalité de l'activitée ${ }^{303}$."

Sans base légale pour justifier une intervention politique, les membres de la fondation évoquent d'autres possibilités pour limiter la pratique de la transplantation. L'édiction de normes de qualité définies par l'ASSM en collaboration étroite avec Swisstransplant est proposée. Il s'agit dans cette optique de s'appuyer sur une institution dont les directives médico-légales, comme la définition des critères de mort cérébrale, sont tacitement reconnues et appliquées. Dans ce

302 Idem, p. 2

303 Correspondance, "Exigences applicables aux hôpitaux autorisés à effectuer des transplantations d'organes", 28 septembre 1992. 
but, des contacts sont pris avec les directeurs d'hôpitaux sans que toutefois des réalisations concrètes en découlent ${ }^{304}$.

La fondation décide de mettre à contribution les groupes de travail pour définir une liste fixe de critères médicaux dans le but d'élaborer des recommandations, comme l'illustre ce courrier du président du Comité exécutif transmis aux représentants des groupes de travail:

«Il nous est donc apparu comme indispensable que des critères médicaux, tels que, par exemple, la formation des équipes, la structure hospitalière, le personnel à disposition et l'expérience passée, soient pris en considération lorsqu'il s'agit d'envisager l'ouverture de nouveaux centres de transplantation. La proposition par les médecins eux-mêmes de règles médicales permettra certainement de mieux contrôler le développement de la transplantation et des centres de transplantation dans notre pays ${ }^{\mathbf{3 0 5}}$.»

Les acteurs impliqués dans la fondation et particulièrement dans le Comité exécutif n’ont aucun bénéfice à entraver l'activité de transplantation à laquelle ils participent par le biais de leur activité professionnelle. Restée à notre connaissance au stade de projet, cette liste n'est pas formellement établie par la fondation. Quelques courriers montrent toutefois la préférence des médecins pour le statu $q u o$. Ainsi Largiadèr suggère dans un courrier transmis au président du Comité d'établir comme critère la nécessité pour l'établissement de réaliser plusieurs types de transplantations, avec exception pour l'hôpital de Saint-Gall ${ }^{306}$.

\section{Restreindre la pratique de la transplantation dans les établissements privés}

Swisstransplant n'est pas dépourvue de moyens de pression sur les cliniques. La structure logistique et organisationnelle mise

304 Correspondance, "Projet de lettre et propositions de Swisstransplant à la Conférence des directeurs cantonaux des affaires sanitaires", 3 octobre 1991.

305 Correspondance, "Élaboration de règles médicales visant à spécifier les caractéristiques des centres habilités à effectuer de la transplantation d'organes", 30 décembre 1992, p. 1.

306 Correspondance, "Anforderungen an Transplantationenzentren", 25 février 1993. 
en place par la fondation permet aux centres de transplantation de bénéficier d'infrastructures pour mener à bien les investigations médicales et de profiter d'un réseau d'échanges d'organes. En refusant de mettre cette structure à disposition de cliniques privées, elle les exclut du groupe de partage de moyens techniques et de ressources. Concrètement le processus d'évincement s'exerce par le refus de collaboration du laboratoire d'histocompatibilité. Les tests de compatibilité tissulaire ne sont effectués que sur les échantillons transmis par les membres des groupes de travail de Swisstransplant. Dans le cas de la transplantation rénale à partir de donneur décédé, les chances de réussite sont largement compromises sans la réalisation de ces tests. La fondation refuse également l'accès aux ressources fournies par la Coordination nationale. De fait, chaque donneur identifié dans les hôpitaux publics étant annoncé à la Coordination, cette mesure vise à empêcher les cliniques privées de recevoir un organe prélevé en Suisse.

Sans base légale permettant d'interdire la pratique des transplantations dans les cliniques, la fondation agit au cas par cas. La mobilisation de leviers politiques reste une des stratégies envisagées, mais se limite à des sollicitations exercées localement. Dans le canton d'Argovie, la Clinique im Schachen souhaite constituer un programme de transplantation rénale à partir de donneur vivant apparenté. Ce type de programme représente une réelle menace puisqu'il peut être développé indépendamment des structures mises en place ou gérées par la fondation. Les dons «vivant apparenté » ne nécessitent pas impérativement les compétences techniques du laboratoire d'histocompatibilité alors que le recours au réseau d'échanges n'entre pas en compte. Face à cette situation inédite, Swisstransplant mobilise des acteurs locaux pour tenter d'empêcher la mise sur pied de ce programme. Largiadèr contacte le président de la fondation, le conseiller d'État genevois Guy-Olivier Segond, en espérant pouvoir profiter de son implication professionnelle pour sensibiliser la CDS à cette problématique. Circonscrire la transplantation aux six centres représente un moyen de garantir selon le chirurgien zurichois la probité de la procédure d'attribution et de provenance de l'organe: 
«J'ai toujours été de l'avis que la transplantation d'organes doit être limitée aux hôpitaux étatiques afin d'exclure avec certitude le danger d'un usage inapproprié et du commerce d'organes ${ }^{307}$. »

Si les courriers sont transmis à plusieurs destinataires, comme le médecin cantonal, le Conseil d'État et des représentants des assurances, les envois font l'objet d'une diffusion par un nombre important d'acteurs divers et au nom d'institutions variées. À titre privé, au nom de la fondation ou d'autres institutions comme la Société de néphrologie, une multitude de courriers est envoyée pour informer largement sur la situation. La démarche atteint son objectif puisque le médecin cantonal argovien adresse un courrier à la clinique. Si la question de l'opacité de la provenance des organes est marginale dans l'argumentaire pour dissuader les partisans de la pratique privée des transplantations, la présentation de l'ensemble des impératifs infrastructurels et médicaux en constitue l'élément central. Maintes fois développé par la fondation, cet argument cible, dans ce courrier, la nécessité de disposer d'une équipe complète et interdisciplinaire, ainsi qu'une expérience en matière de transplantation, que seuls les centres possèdent:

"Cela ne suffit pas d'avoir à disposition un néphrologue et un urologue "transplanteur" pour assurer un service pour parer aux complications éventuelles (365 jours par année). Les transplantations à partir de donneurs vivants sont menées dans les six centres de transplantation de Swisstransplant alors que les équipes ont acquis une large expérience durant de nombreuses années dans le domaine de la transplantation de reins de cadavre. Dans la clinique Schachen, il manque une couverture dans les disciplines voisines immédiates (entre autres les tests d'histocompatibilité réalisés selon les standards de qualité d'un laboratoire de Swisstransplant, l'infectiologie, l'angiologie et la médecine nucléaire, etc.) ${ }^{308}$.»

Dans d'autres cas, il arrive que des acteurs se mobilisent pour tenter de modifier les lois cantonales pour permettre de disposer,

307 Correspondance, "Nierentransplantationen mit Lebendspender in einer Privatklinik", 17 octobre 1994 [notre traduction].

308 Correspondance, "Projekt Verwandten nieren Transplantationen", 12 avril 1995 [notre traduction]. 
localement, d'une base légale. À Genève par exemple, Morel contacte le médecin cantonal, également membre du Grand Conseil. Le but de cette démarche est de lui faire parvenir l'opinion de personnes impliquées dans la transplantation à Genève concernant la réalisation de cette pratique en milieu privé ${ }^{309}$. Les conclusions du groupe genevois sont transmises directement au Conseil d'État par le médecin cantonal qui y présente son rapport ${ }^{310}$.

La limitation du nombre de centres ne trouve pas de solution définitive, mais la thématique disparaît au milieu des années 1990, sans doute avec le renoncement tacite des cliniques privées à développer des programmes de transplantation. La configuration hospitalière de la transplantation reste fixée aux établissements universitaires, qui, en l'absence de législation, gardent implicitement la prérogative des transplantations.

\section{Concentrer et limiter les programmes de transplantation}

Les programmes de transplantation de chaque centre se sont développés, pour le rein dès la fin des années 1960, pour les autres organes dès la fin des années 1980, sans que la répartition des types de transplantations entre établissements joue un rôle prépondérant dans la configuration interne de l'offre thérapeutique. Cette répartition est discutée souvent bien après la mise en place des programmes, comme l'illustre un courrier transmis par un Conseil d'État bernois à son homologue bâlois en 1990. Il y propose l'élaboration d'un accord intercantonal pour que les patients bâlois en besoin d'une greffe de foie soient dirigés à Berne. Alors que le programme est en activité depuis $1983^{311}$, il s'agit pour le conseiller d'État bernois de faire de l'Inselspital un «centre régional pour les transplantations hépatiques ${ }^{312}$ » par

309 Correspondance, "Autorisation d'effectuer des transplantations en cliniques privées, projet de loi à Genève", 22 décembre 1992.

310 Correspondance, "Pratique de la transplantation en cliniques privées", 22 février 1993.

311 Correspondance, "Collaboration intercantonale dans le domaine de la chirurgie de transplantation", $1^{\text {er }}$ juin 1990, p. 2.

312 Idem, p. 1. 
l'entremise d'un "rattachement durable des cantons du nordouest de la Suisse ${ }^{313} »$.

À partir du milieu des années 1990, dans le processus général de clarification des pratiques d'attribution des organes et de mise en liste d'attente des patients dans les centres, la question du type de programme par centre prend une importance inédite. La nécessité de présenter un fonctionnement le plus transparent possible dans le cadre des négociations avec la Confédération pèse largement sur l'émergence de ces réflexions. Une consultation des groupes de travail et des représentants des centres est effectuée par le Comité exécutif. Le but est de créer des normes ou des critères définissant le nombre de centres nécessaires par organe selon des zones géographiques. Établis directement dans les centres par les médecins, ces critères contribuent à une autolimitation. Comme l'illustre l'extrait d'un courrier, ce processus de consultation est fondé sur la crainte de voir des acteurs extérieurs au domaine de la transplantation s'emparer de cette problématique:

"Avant que des décisions restrictives en défaveur des malades ne nous soient imposées, il est indispensable que nous nous mobilisions pour émettre nos recommandations ${ }^{314}$.»

Certainement peu pressés d'entamer des réflexions visant à entraver les possibilités de développer des programmes, les acteurs médicaux des centres ne donnent, à notre connaissance, aucune réponse formelle à la demande transmise en 1995 par le Comité exécutif.

Difficile pour Swisstransplant d'influencer directement le fonctionnement interne des centres de transplantation. Bien qu'intégrés dans les différentes structures de la fondation par le biais des représentants des centres et des groupes de travail, les hôpitaux restent libres de mettre en place de nouveaux programmes de transplantation. Leur activation passe par l'intégration dans le groupe de travail de Swisstransplant d'un représentant du centre dans lequel le nouveau programme est mis sur pied. Ce processus ne fait pas

313 Idem.

314 Correspondance, "Limitation des programmes de transplantation par centre en Suisse, prochain Conseil de fondation de Swisstransplant", 22 mai 1995. 
l'objet d'un protocole établi, mais semble être informellement réglé par les praticiens eux-mêmes.

Hiérarchiquement, les groupes sont subordonnés au Conseil de fondation qui a la charge de les organiser ${ }^{315}$. C'est ce lien de subordination qui est invoqué pour justifier le décret d'un moratoire sur l'entrée de nouveaux centres dans les groupes de travail de Swisstransplant en 1999.

Dans le contexte politique d'élaboration de la loi sur la transplantation qui génère une clarification générale des pratiques et des statuts, la question du nombre de centres est donc tranchée provisoirement par une décision du Conseil de fondation. Imposée aux centres de transplantation, cette décision est diffusée ainsi des structures dirigeantes vers les entités subordonnées.

Ce qui s'apparente à un geste fort de la part du Conseil de fondation vise à sauvegarder les acquis de la configuration hospitalière de la transplantation. Il s'agit pour Swisstransplant d'éviter une diminution de la densité du réseau. Plusieurs propos relatés dans des procès-verbaux attestent une crainte de voir l'équilibre remis en cause comme l'illustre cet exemple:

«[...] la Suisse possède, en comparaison avec l'étranger, une haute densité de centres de transplantation pour le cœur, poumon et foie. Les organismes payeurs et une large frange du secteur politique considèrent cette densité trop haute et soutiennent une concentration ${ }^{316}$.»

Même en l'absence d'un plan directeur initialement élaboré entre les centres, la répartition des types de transplantations est restée stable depuis la fin des années 1980. Cet équilibre tacite semble remis en cause par la volonté affichée de certains établissements d'étoffer leur offre thérapeutique à la fin des années 1990. Si l'éventualité de la mise en place d'un moratoire est évoquée dans le cadre du développement d'un programme de transplantation pancréatique à Berne en $1998^{317}$, la question prend la forme d'un débat plus marqué en 1999. Par le biais d'une lettre transmise à Swisstransplant, l'hôpital

315 P-V CF, 11 novembre 1998, annexe 11.

316 P-V CF, 11 novembre 1998, annexe 11 [notre traduction].

317 P-V CF, 10 mai 1999, p. 7. 
de Berne informe que son offre thérapeutique va également s'étendre à la transplantation combinée cœur-poumon ${ }^{318}$. Ce courrier fait l'objet d'une discussion entre les membres du Conseil de fondation concernant la nécessité ou non de limiter les programmes de transplantation par centre. Si la limitation du nombre de centres à la configuration actuelle fait l'unanimité parmi les membres, définir un nombre de programmes pour chaque centre est sujet à controverses. L'élaboration d'une politique restrictive est perçue par certains comme une entrave posée à la recherche dans les hôpitaux. En effet, la mise sur pied d'un programme génère des bénéfices scientifiques et médicaux qui profitent à l'ensemble de l'établissement ${ }^{319}$. Pour les partisans, il s'agit d'empêcher des dépenses supplémentaires dans un contexte médical où les besoins des patients sont couverts ${ }^{320}$. Les dissensions semblent superficielles, permettant la proposition formelle d'un moratoire à la suite des discussions lors de la même réunion. Dans un contexte politique où l'obligation de rendre l'organisation de la transplantation plus transparente se fait largement ressentir par Swisstransplant, ces deux événements jouent un rôle déclencheur.

Formellement, ce moratoire empêche les transplanteurs des hôpitaux de mettre sur pied des programmes à leur guise, mais les contraint à obtenir l'autorisation du Conseil de fondation. Dans la pratique, il ne prend pas une forme totalement inflexible. Par exemple à Zurich, la réactivation d'un programme de transplantation d'îlots pancréatiques momentanément arrêté fait l'objet d'une demande des médecins. Adressée au préalable informellement à un membre du Conseil de fondation dans le but d'obtenir quelques informations complémentaires sur la procédure à suivre, cette demande contourne la voie officielle que devrait ponctuer l'approbation du Conseil de fondation. Comme le laisse comprendre la réponse transmise aux transplanteurs zurichois, il ne s'agit pas formellement du développement d'un nouveau programme:

318 P-V CF, 13 janvier 2000, p. 3.

319 Idem.

320 Idem. 
«L'université de Zurich est évidemment libre d'inscrire à n'importe quel moment des patients pour la transplantation de pancréas et d'îlots sur la liste d'attente de Swisstransplant, puisque Zurich est un centre reconnu pour la transplantation du pancréas depuis la création de Swisstransplant ${ }^{321}$.»

L'enjeu n'est pas l'élaboration technique du programme, mais bien la possibilité pour les transplanteurs d'inscrire les patients sur la liste d'attente commune de la Coordination nationale.

\subsection{Conclusion}

L'influence de Swisstransplant sur la transplantation entre les années 1985 et 2000 se traduit par la mise en place d'un système de gestion des échanges d'organes. Pratique médicale jusque-là peu normée, la transplantation devient ainsi une organisation unifiée afin de permettre une utilisation efficiente des organes disponibles. Si l'amélioration de la maîtrise des aspects biologiques ou techniques joue un rôle incontestable, les mécanismes de régulation mis en place autour de cette pratique ne sont pas moins importants. Par l'intégration de représentants de chaque organe et de chaque centre dès la fin des années 1980, Swisstransplant construit un modèle d'échange dans lequel le lien entre l'organe et l'hôpital est très étroit. Malgré la supervision unifiée des activités par la Coordination nationale créée en 1992, les hôpitaux conservent une influence dans l'orientation des pratiques grâce à l'activité des groupes de travail chargés d'élaborer les règles d'échange et de répartition des organes entre les centres. D'abord dépendante des centres, isolément les uns des autres, elle devient la pratique médicale d'une communauté, celle des transplanteurs.

Face aux institutions extérieures au réseau ainsi constitué, la fondation mobilise des moyens pour empêcher la réalisation d'interventions hors de son contrôle. Aussi, elle régule les transplantations au sein de son réseau et s'érige comme l'entité de référence dans la gestion de ces pratiques. L'importance du corps médical dans

321 Correspondance, "Inseltransplantations-Programmam Universitätsspital Zurich", 30 juin 1999, p. 2 [notre traduction]. 
la définition des règles et dans l'organisation des transplantations conduit toutefois à une remise en cause du modèle de répartition des organes. Face aux pressions extérieures, la fondation limite la marge de manœuvre des médecins en statuant sur l'élaboration de nouveaux programmes de transplantation par centre. 



\title{
Chapitre 4
}

\section{Les questionnements éthiques sur la transplantation dans les revues médicales (1950-1980)}

\begin{abstract}
Alexia Cochand
Jusqu'à l'usage généralisé de la cyclosporine dans les années 1980, le développement clinique des transplantations est freiné par la gestion difficile du rejet. Pourtant, entre les années 1950 et 1980, la transplantation fait l'objet, sous l'impulsion de médecins actifs de quelques hôpitaux occidentaux, de recherche et d'expérimentation clinique appliquées à la plupart des organes solides ${ }^{322}$. Si l'utilisation du rein artificiel associée à l'élaboration de protocoles médicamenteux facilitent la mise en place des programmes de transplantation rénale dans la plupart des établissements hospitaliers occidentaux de pointe à partir de la fin des années 1960, des difficultés immunologiques identiques caractérisent les interventions menées par les médecins dès les années $1950^{\mathbf{3 2 3}}$.

Dans un contexte thérapeutique où la survie des patients est faible mais porteuse d'espoirs, les contraintes médico-techniques liées au besoin d'organes vascularisés soulèvent des enjeux médicaux
\end{abstract}

322 Hamilton 2012, 269-295.

323 Sur les difficultés auxquelles se heurtent les pionniers de la transplantation rénale dans les années 1950, voir Küss et Bourget 1992, 39-50. 
inédits, tels que la réalisation de prélèvements sur des donneurs vivants ou l'élaboration de critères pour définir la mort cérébrale. Aussi, on peut se demander dans quelle mesure le développement de la transplantation, caractérisée par des procédures médicotechniques spécifiques, s'accompagne de questionnements éthiques. La dimension alors largement expérimentale de la transplantation fait-elle l'objet de justifications de la part des transplanteurs? Le recours à des donneurs vivants suscite-t-il des discussions? La mort cérébrale bénéficie-t-elle de la reconnaissance unanime du corps médical non spécialisé ? Quand et comment la thématique du manque d'organes est-elle abordée alors que les transplantations sont réalisées dans un petit nombre d'hôpitaux?

L'examen de ces questions permet d'évaluer le caractère historiquement inédit de certaines problématiques médicales, sociales et politiques actuelles, telles que la pénurie ou le mode de consentement, et de les inscrire dans un cadre de réflexion élargi. Nous avons retenu une acception générale de la notion de "questionnements éthiques ", incluant toutes les réflexions et préoccupations morales, économiques ou philosophiques qui se distinguent de problématiques strictement médicales et scientifiques ${ }^{\mathbf{3 2 4}}$. Nous avons choisi de mener cette analyse en nous intéressant au contenu de revues médicales qui constituent l'outil de diffusion du savoir médical par excellence dans la période considérée ${ }^{325}$. Il s'agit d'identifier dans quelle mesure les questionnements éthiques profitent de ce «relais». Plus précisément, nous utilisons comme base documentaire non exhaustive les articles relatifs à la transplantation d'organes relevés dans quatre revues médicales suisses généralistes: le Bulletin des médecins suisses (BMS), la Revue médicale de la Suisse romande (RMSR), Médecine et Hygiène (MßH) et Praxis: Schweizerische Rundschau für Medizin ${ }^{326}$. L'utilisation de revues généralistes plutôt

324 Pour cette définition, nous nous inspirons de Cameron 2002.

325 Renaudet 2011.

326 Nous avons compulsé tous les numéros des quatre revues pour la période entre 1950 et 1980 pour identifier l'ensemble des articles abordant la transplantation d'organes. Nous avons ensuite constitué notre corpus en retenant uniquement les articles, quelle que soit leur forme journalistique, contenant des éléments éthiques. Selon ces 
que de périodiques spécialisés vise à saisir une large amplitude d'avis formulés par des médecins aux parcours professionnels variés. Dans une optique comparative, ce choix permet de distinguer les réactions des transplanteurs de celles des médecins étrangers au domaine de la transplantation. Dans un premier temps, nous présenterons les revues et leurs caractéristiques éditoriales. À cet égard, nous évaluerons le potentiel informatif de chacune des revues sur la transplantation en fonction de la ligne éditoriale et du type d'articles dans lesquels cette thématique est abordée. Dans un second temps, nous mettrons en évidence les différents thèmes du développement de la transplantation qui suscitent, à des degrés variables, des questionnements éthiques en suivant un axe chronologique, des années 1950 à la fin des années 1970.

\subsection{Les revues médicales en Suisse}

En l'absence d'association professionnelle nationale compétente dans la prise en charge de problématiques éditoriales et scientifiques de la presse médicale en Suisse, les revues publiées dans les années 1950 se sont le plus souvent constituées informellement autour de groupes de médecins. Réparties par région linguistique, les revues assurent leur propre fonctionnement grâce au bénévolat des membres du comité de rédaction. Hospitaliers ou indépendants, les médecins se partagent l'écriture d'articles et la gestion de rubriques en fonction du domaine d'expertise scientifique de chacun. Aussi les thématiques abordées dans les revues sont souvent variées et destinées au corps médical dans son ensemble. Si l'émergence de nouvelles spécialités médicales dans les années 1950 conduit à la création d'entités administratives apparentées, la rareté d'un lectorat potentiel "spécialisé »sur le territoire et la concurrence avec des revues étrangères prestigieuses limitent le processus de fragmentation et de spécialisation de la production de publications périodiques. La publication d'informations médicales de qualité,

critères, le nombre global d'articles constitutifs de notre corpus s'élève approximativement à 200 (environ 30 dans le BMS, 40 dans Praxis, 120 dans M\&H et 10 dans la RMSR). 
représentant le plus exhaustivement possible les spécialités médicales variées, constitue ainsi un enjeu scientifique pour les comités de rédaction des revues généralistes.

Si les revues médicales visent à transmettre des informations sur la pratique de la médecine, la ligne éditoriale qu'elles poursuivent en modifie la substance. Sans en être investies officiellement par la Fédération des médecins suisses (FMH), MひH, Praxis et la $R M S R$ ont pour objectif la formation continue du médecin par le biais d'un contenu éditorial identique. Composé d'articles originaux, revues de presse, comptes rendus de conférences, recensions d'ouvrages, communiqués officiels ou annonces de manifestations, le contenu varie en fonction d'éléments scientifiques et matériels liés à la spécificité du contexte de production de chaque revue. Les moyens logistiques à disposition, le public médical visé ou encore la zone géographique de publication en constituent des exemples particulièrement influents.

Publiée à un rythme hebdomadaire, $M \mho ̛ H$ est l'une des rares revues helvétiques dédiées à la médecine qui possède un poste de rédacteur en chef à temps complet dès sa création en $1943^{327}$. Au-delà des tâches de coordination et de constitution des numéros, il participe activement à l'élaboration du contenu par la composition d'éditoriaux, de comptes rendus de conférences ou encore de billets d'humeur. À ce titre, Pierre Rentchnick, qui assure cette fonction à partir de 1953, constitue une figure omniprésente de la revue. Outre un conseil de rédaction à Genève, la revue genevoise possède un réseau de correspondants à l'étranger de formation médicale qui offre à ses lecteurs des articles sur les recherches menées dans des centres universitaires internationaux. Forte de ces moyens logistiques, la ligne éditoriale est aussi bien tournée vers la formation continue du médecin par la publication d'articles scientifiques que vers l'information relative à des événements médicaux. À l'opposé, la $R M S R$, revue mensuelle dont la première édition remonte à 1881 , se caractérise par une large couverture de la pratique médicale régionale. Destinée à l'ensemble des membres de la Société médicale de la Suisse romande, elle propose essentiellement des articles 
originaux rédigés par des médecins actifs dans des établissements sanitaires romands sans exclure les publications de généralistes. À côté des rubriques dont les membres du comité se répartissent la charge, l'élaboration d'un numéro habituel s'effectue sans doute informellement moyennant la sollicitation de connaissances ou de collègues à qui confier la rédaction d'articles.

Publiée depuis 1923 sous l'impulsion d'un groupement de praticiens de l'hôpital de Zurich, Praxis se caractérise par l'omniprésence de la médecine spécialisée hospitalière. Son comité de rédaction est constitué de médecins en activité dans les différents établissements universitaires de toute la Suisse. Parallèlement à la publication d'articles originaux selon le principe de "l'évaluation par les pairs", un numéro habituel, publié chaque semaine, propose une vaste revue de presse dont l'élaboration est confiée à différents rédacteurs en fonction de critères comme la langue d'édition originale dans laquelle sont présentées la recherche et les compétences de chacun. Classées par spécialité médicale et signées par l'unique patronyme de l'auteur, sans mention d'un domaine d'expertise ou de l'institution qui l'emploie, ces notices permettent aux lecteurs de prendre connaissance des recherches et travaux en cours dans des hôpitaux internationaux. Si la revue semble donc principalement destinée à un public médical restreint, elle assure suivant un rythme variable la publication de comptes rendus de la société cantonale des médecins de Zurich et de Berne.

Le Bulletin des médecins suisses, quatrième revue de notre corpus, propose une ligne éditoriale atypique en se concentrant sur des thématiques liées à la politique professionnelle et à la défense des intérêts des praticiens. Son statut d'organe officiel de la FMH lui permet de disposer de moyens financiers qui conduisent progressivement à la professionnalisation de postes au sein du comité de rédaction et des différentes structures affiliées. Un temps plein est créé en 1964 avec la mise en place du service d'information qui vise à renseigner les médecins sur les questions d'actualité en lien avec l'exercice de la médecine notamment en publiant ponctuellement des notices informatives dans le périodique. Le BMS représente également un canal de diffusion privilégié pour les directives et positionnements de groupements ou d'institutions sur des problématiques médicales 
puisque la revue est adressée à l'ensemble des membres affiliés à la FMH. Comme les autres revues, un numéro standard est constitué d'articles que les membres de la rédaction se répartissent, mais propose aussi la publication de lettres ouvertes sur des thématiques concernant la pratique de la médecine.

\subsection{La transplantation dans les revues: panorama chronologique}

Si le contenu des notices éditoriales et des articles originaux est largement influencé par la ligne de publication suivie par les revues, le niveau de maîtrise médico-technique des médecins et l'actualité de la mise en œuvre de la transplantation dans le contexte sanitaire suisse en modifient la répartition chronologique. La transplantation est ainsi d'abord uniquement relayée par le biais de recensions d'articles relatifs aux expérimentations menées à l'étranger dans les années 1950 et le milieu des années 1960. Dans la seconde moitié des années 1960, l'organisation de conférences en Suisse axées sur les problématiques juridiques en lien avec la pratique de la transplantation fait l'objet de plusieurs comptes rendus. Reflets de l'intérêt croissant que les enjeux médicaux et juridiques soulevés par les prélèvements suscitent au sein de la communauté médicale helvétique, ces publications émanent surtout du BMS. Moyen privilégié de suivre les enjeux médicaux et logistiques autour du développement des transplantations, les articles scientifiques ou rédigés par des médecins impliqués dans ce domaine font principalement leur apparition après la mise en place de programmes de transplantation rénale dans les hôpitaux suisses au début des années 1970. Il convient donc de suivre de manière plus détaillée chronologiquement le développement de la transplantation dans les revues médicales généralistes.

\section{La transplantation à l'étranger: revues de presse et commentaires}

Alors que la transplantation rénale se développe dans les hôpitaux à Paris et à Boston dès le début des années 1950, elle fait l'objet 
d'un intérêt éditorial contemporain dans Praxis et $M \mho H^{328}$. C'est par l'intermédiaire de sections consacrées à la revue de presse que les lecteurs sont renseignés sur ces expérimentations et leurs résultats. Centrées sur la présentation d'informations liées à l'actualité scientifique et médicale inédite, ces rubriques mettent en évidence des travaux de recherche pointus. Ainsi, elles ne contiennent pas de recensions d'articles liés aux enjeux logistiques soulevés par l'émergence des programmes de transplantation. Dans Praxis, la structure interne de la rubrique présente diverses spécialités médicales, dans un format bref, le plus souvent composé d'extraits de l'article original, complété par un commentaire de quelques lignes. Dans $M \mho H$, les informations relatives aux transplantations rénales sont relayées dans la chronique "acquisitions thérapeutiques ». Élaborée autour d'une sélection subjective de travaux publiés dans des revues internationales durant l'année écoulée, cette rubrique est organisée par spécialité. Comme dans Praxis, les investigations conduites dans le domaine de la transplantation rénale sont abordées dans les sous-rubriques «néphrologie» ou "urologie».

Le caractère expérimental que conserve l'ensemble de la transplantation, à l'exception du rein, jusqu'à la fin des années 1970, contribue sans doute à la diffusion inconstante des résultats obtenus par les équipes médicales concernées. Les notices qui s’y rapportent se limitent aux activités expérimentales exécutées par de rares pionniers. À ce titre, en 1968 et en $1971^{329}$, les résultats présentés par Thomas Starzl et son équipe dans le domaine de la transplantation hépatique sont relayés dans Praxis. En 1970 et 1979, deux notices relatives aux travaux menés par Norman Shumway de Stanford concernent la transplantation cardiaque ${ }^{330}$.

L’omniprésence de notices éditoriales indirectes dans notre corpus s'explique par la volonté des «transplanteurs» de publier dans des périodiques internationaux spécialisés pour traiter de problèmes

328 Sur le traitement de la thématique de la transplantation dans ces deux revues, voir Cochand 2015.

329 Praxis, 43, 1969, 1386; Praxis, 60, 1971, 1621.

330 Praxis, 21, 1970, 781-782; Praxis, 68, 1979, 1129. 
médicaux pointus ou dans des revues généralistes éditées dans le lieu où ils exercent pour présenter la mise en place de programmes. Doté d'un rayonnement scientifique dans un espace régional qui s'étend jusqu'en France et en Belgique, MÆH publie en 1967 un article original du pionnier belge de la transplantation rénale cadavérique, Guy Alexandre ${ }^{331}$, et la présentation d'une transplantation pulmonaire effectuée à l'hôpital de Gand en $1969^{332}$. La mobilisation des réseaux de connaissances constitue également un moyen informel de favoriser la publication ponctuelle d'articles dans les périodiques. Sur sollicitation du rédacteur en chef, Charles Dubost relate dans la revue genevoise la première transplantation menée en France, réalisée avec son équipe le 12 mai 1968 sur le père Boulogne, avant même que les publications paraissent dans La Presse médicale en août $1968^{333}$.

\section{Les aspects juridiques: comptes rendus de conférences et communiqués officiels}

L'attention portée à la réglementation juridique de la transplantation s'affirme dans les revues dès le milieu des années 1960 au moment où la greffe rénale fait son apparition dans les hôpitaux suisses. La parution dans le BMS de plusieurs comptes rendus d'allocutions prononcées par des juristes marque l'intérêt que suscitent pour les praticiens les problématiques juridiques induites par l'essor de la transplantation. Ainsi en 1967, la société médicale du canton de Berne propose pour sa réunion d'été une conférence sur les questions éthiques soulevées par le perfectionnement des techniques de réanimation, dont la partie exclusivement consacrée à la transplantation est publiée ${ }^{334}$. L'intérêt heuristique de ces comptes rendus est double: ils permettent d'une part de mettre en évidence le lien causal établi dans l'esprit des médecins entre l'avènement de techniques de suppléance des fonctions vitales et la réalisation de

$331 \mathrm{MEH}, 17$ mai 1967, 527-528.

332 M\&H, 14 mai 1969, 557-560.

333 M\&H, 15 juillet 1968, 811-812.

334 Bulletin des médecins suisses (BMS), 33, 1968, 873-877. 
transplantations, d'autre part d'évaluer dans quelle mesure cette thématique est reliée au contexte sanitaire helvétique.

La première transplantation cardiaque (considérée comme un succès) réalisée le 4 décembre 1967 au Cap en Afrique du Sud intensifie l'intérêt vis-à-vis de la réglementation de la transplantation. Ainsi, quelques mois après l'événement, le $B M S$ fait paraître un résumé d'un colloque organisé en mars 1967, à Londres, par la Ciba Foundation, sans écho dans les revues au moment où il se déroule. Créée par l'entreprise pharmaceutique Ciba dans le but de promouvoir la coopération internationale dans le domaine de la biologie, la chimie et la médecine par le biais de symposiums annuels, cette fondation rassemble des juristes et des spécialistes médicaux. Au travers de conférences et de discussions, ils abordent entre experts la question du don vivant, de la mort cérébrale et de problématiques médico-juridiques liées à la transplantation ${ }^{335}$. Entre l'année 1968 et le début des années 1970, plusieurs institutions médicales ou paramédicales suisses et internationales prennent officiellement position ou publient des directives relatives aux critères de la mort cérébrale et aux infrastructures nécessaires à la réalisation des transplantations. Largement diffusé au sein de la communauté médicale nationale, le BMS relaie ces informations, en mettant en exergue les directives de l'ASSM sur la définition de la mort cérébrale en 1969 et la notice de l'Association pour la chirurgie de transplantation, du groupe de travail pour l'histocompatibilité et de la commission pour les transplantations rénales de la Société de néphrologie concernant les besoins et exigences dans le cadre de la création d'un centre de transplantation rénale ${ }^{336}$. Parallèlement au regard sur la réglementation en Suisse qu'apporte le $B M S, M \mho H$ se fait l'écho du positionnement d'organisations internationales ou étrangères jugées influentes par le comité de rédaction. Elle diffuse les prescriptions établies par la Société allemande de chirurgie ${ }^{337}$ en 1967 ou la Société internationale de transplantation ${ }^{338}$ en 1971.

335 BMS, 20, 1968, 519-522.

336 BMS, 29, 1970, 853.

337 M\&H, 24 avril 1968, 497.

338 M\&H, 26 mai 1971, 965. 


\section{Les premières transplantations cardiaques dans Médecine et Hygiène}

Forte de l'implication d'un rédacteur en chef employé à plein temps, $M \nLeftarrow H$ est l'unique revue du corpus à faire paraître des notices éditoriales informatives sur les premières transplantations cardiaques menées entre la fin de l'année 1967 et la fin des années 1970. Le plus souvent dépourvues de références bibliographiques, ces notices sont centrées sur la dimension sensationnelle des événements rapportés.

Dans les numéros chronologiquement proches de l'intervention menée par Christiaan Barnard au Cap en Afrique du Sud en 1967, des rubriques inédites, "échos et nouvelles ", "la médecine en marche » et "information", deviennent les espaces de publication destinée à la transmission d'informations hétéroclites. Par exemple, des articles de presse du Monde et du Figaro ${ }^{339}$, un éditorial de l'organe officiel du Vatican ${ }^{340}$ ou encore le protocole opératoire de l'intervention conduite au $\mathrm{Cap}^{\mathbf{3 4 1}}$ y sont reproduits. Les nombreuses tentatives de transplantation qui marquent les années 1968 et 1969 y trouvent une place au travers des notices, le plus souvent de quelques lignes, où l'absence d'informations médico-techniques avérées est compensée par une brève analyse du rédacteur en chef de $M \mho H$. En marge des indications factuelles, l'opération menée par Shumway en janvier 1968 fait ainsi l'objet d'un compte rendu succinct par Rentchnick sous le titre «Le patron entre en scène »:

«[...] On ne peut s'attendre techniquement à des différences entre les greffes de cœur pratiquées au Cap et à Palo Alto, puisque la même technique a été utilisée, c'est à nouveau le problème du traitement immunosuppresseur qui va se trouver au premier plan $^{342}$.» La première transplantation cardiaque réalisée à Paris en mai 1968 donne lieu à un article accompagné de la photographie et du nom du patient. Les prouesses du chirurgien en question constituent un

$339 \mathrm{M} \& H, 17$ janvier 1968, 80.

340 M\&H, 21 février 1968, 204.

341 M\&H, 31 janvier 1968, 120.

342 M\&H, 10 janvier 1968, 34. 
élément central des propos, participant à la diffusion d'une image presque héroïque, comme l'illustre ce passage:

«[...] C'est alors la réussite d'une série de "premières" qui vont classer incontestablement le professeur Dubost parmi les meilleurs spécialistes européens: en 1951, le premier cas de greffe d'un anévrysme à l'aorte abdominale; en 1950, le premier cas de résection d'un anévrysme sacciforme de l'aorte ascendante; en 1953, une dilatation instrumentale de sténose mitrale; en 1959, le premier cas de désoblitération coronarienne pour coronarite ostiale; en 1967, la première hétérogreffe en position mitrale; enfin, le 12 mai 1968, une greffe de cœur chez un homme. Il fut le premier, en France, à pratiquer la chirurgie à cœur ouvert ${ }^{343}$.»

Réalisée en marge de la deuxième conférence internationale sur les transplantations, à laquelle le rédacteur en chef prend part en qualité d'envoyé spécial, une série d'entretiens menés avec des transplanteurs est publiée sous une rubrique inédite, «le Dr [...] m'a dit ». Si le contenu des interviews présente un intérêt heuristique en soi, la création de cette rubrique témoigne de l'aura de ces praticiens et la curiosité qu'ils suscitent au sein de la communauté médicale, du moins pour le rédacteur de la revue. En préambule à l'entretien conduit avec Denton Colley, particulièrement actif à l'institut cardiologique de Houston, Rentchnick mentionne ainsi qu'il "a déjà réalisé 11 transplantations cardiaques en quatre mois, dont 3 en l'espace de cinq jours. Huit de ces malades sont en vie et deux d'entre eux ont déjà repris une activité professionnelle ${ }^{344}$ ».

L'abandon des transplantations par la plupart des équipes médicales dès le printemps 1969 marque la fin progressive de son attrait «sensationnel» dans la revue genevoise.

\section{Les articles scientifiques originaux: la transplantation en Suisse}

Si la connaissance sur les activités médicales liées à la transplantation dans les hôpitaux au niveau international dont dispose le lec-

343 M\&H, 15 juillet 1968, 811.

$344 \mathrm{M} \& \mathrm{H}, 16$ septembre 1968, 1032. 
torat dépend du travail éditorial des comités de rédaction des revues, les informations relatives à la pratique de ce type d'interventions en Suisse sont assurées par la diffusion d'articles scientifiques originaux. Sous forme d'une présentation d'activité, les équipes médicales y mettent en lumière les résultats obtenus en évoquant également des aspects techniques et organisationnels comme la procédure opératoire suivie, la provenance de l'organe ou les critères sur lesquels s'est établi le choix du receveur. Jusqu'au milieu des années 1980, le remplacement du rein est l'unique type de transplantation à faire l'objet d'articles originaux. Toutefois, les communications que publient les revues permettent une connaissance fragmentaire de l'activité clinique des centres suisses dans ce domaine. Moyennant un lien éditorial direct avec $M \nLeftarrow H$, la clinique de chirurgie de Genève propose deux articles, en 1972 et 1978, sous forme de bilan ${ }^{345}$. Concernant l'hôpital de Berne, la RMSR propose en 1971 l'unique panorama relatif aux activités qui y sont menées dès 1969. L'expérience acquise par les hôpitaux de Lausanne, Bâle et Saint-Gall reste inconnue, suggérant l'absence de publication par les équipes médicales pour divers motifs indéterminés, comme la prise en charge d'un nombre trop faible de patients pour constituer une source de comparaison significative. Les activités de l'hôpital cantonal de Zurich, au bénéfice de l'expérience la plus ancienne dans le domaine de la transplantation rénale en Suisse, comme dans celle du pancréas où la première transplantation est réalisée en $1976^{346}$ par Felix Largiadèr et son équipe, passent largement inaperçues dans les revues de notre corpus. On peut supposer que les équipes zurichoises ont privilégié la publication d'articles dans des revues spécialisées ou d'autres revues médicales suisses généralistes comme la Schweizerische Medizinische Wochenschrift. La première transplantation réalisée à Zurich fait l'objet d'une notice informative uniquement dans $M \mho H$. Par le biais d'une rubrique destinée à la transmission rapide de l'information, le croquis de l'opération est publié à côté d'un bref commentaire ${ }^{347}$.

345 M\&H, 21 juin 1972, 982-986; M\&H, 21 juin 1978, 2337-2338.

346 www.50jahretransplantation.usz.ch (consulté en 2019).

347 M\&H, 23 avril 1969, 504-505. 
Hors des hôpitaux universitaires, Praxis et la $R M S R$ se révèlent être des indicateurs précieux pour comprendre les problématiques auxquelles sont confrontés les médecins. L'activité du centre de néphrologie et de dialyse de l'Ospedale Civico de Lugano et les rapports qu'il entretient avec l'hôpital de Zurich, où les patients tessinois sont transplantés, permettent de suivre dans une publication de 1975 les enjeux médicaux de la prise en charge des transplantés avant et après l'opération ${ }^{348}$.

\subsection{Les questionnements éthiques}

Alors que les premières transplantations cliniques menées à partir des années 1950 sont évoquées dans les revues de presse de Praxis et $M \nLeftarrow H$, avant de faire l'objet d'articles scientifiques avec les premiers programmes de transplantation dans les hôpitaux suisses, les questionnements "éthiques" que suscite leur développement apparaissent liés à l'obtention d'organes. Le donneur vivant apparenté et le patient en état de coma dépassé, requalifié officiellement dès 1968 de «mort cérébrale », constituent successivement, en fonction des moyens techniques et des connaissances immunologiques, les principales sources d'organes. Malgré la mort rapide des patients, le bénéfice thérapeutique des transplantations est peu contesté au sein de la communauté médicale durant la période envisagée. Ainsi, en dépit d'une mise en œuvre limitée par des difficultés liées à la gestion du rejet, le manque d'organes est envisagé comme une menace à l'extension des transplantations dès les années 1950 pour se renforcer à la fin des années 1960 avec la généralisation du prélèvement cadavérique. Comme nous le détaillons dans les pages qui suivent, les questionnements éthiques dans notre corpus concernent principalement le bien-fondé du prélèvement, la mort cérébrale, la justification des transplantations et le manque d'organes.

\section{Légitimer le prélèvement}

Si le savoir-faire opératoire représente une condition nécessaire à la réalisation d'une transplantation, celle-ci dépend avant tout de la 
disponibilité d'organes. Contrairement à certains tissus, comme la cornée, ou au sang, la constitution d'une banque d'organes solides est entravée par des difficultés médico-techniques insurmontables, liées à la vascularisation des organes. Dépendante de la disponibilité d'un donneur, la réalisation d'une transplantation se caractérise ainsi par une organisation non planifiable qui rend son développement clinique difficile. Pour limiter l'imprévisibilité des interventions, les premières équipes constituées autour des chirurgiens français René Küss, Charles Dubost et Monceau Servelle privilégient l'utilisation de reins dont le prélèvement est planifiable. Entre 1951 et 1952, les transplantations sont réalisées avec des organes retirés à des patients pour des raisons thérapeutiques, le plus souvent urologiques, et à des condamnés à mort. Dans le cas des interventions mobilisant les reins d'individus néphrectomisés, la provenance des organes passe inaperçue dans les revues. Les prélèvements effectués sur les suppliciés suscitent en revanche des allusions de nature "éthique " de la part des rédacteurs. Gentil, médecin dont on ne connaît que le patronyme, évoque dans Praxis les avis émis hors de la sphère médicale par des juristes qui assimilent ces prélèvements à une forme de "profanation» ou à un acte de «légitime défense de la société ${ }^{349}$ ". À l'inverse, Gentil envisage ce type de procédure comme un acte médical qui ne diffère en rien de la pratique habituelle des autopsies. De son point de vue, le prélèvement est strictement à distinguer des circonstances dans lesquelles les organes sont mis à disposition. Comme il le précise, "de fait, le fond du problème se trouve bien plus dans la légitimité de la peine capitale ${ }^{\mathbf{3 5 0}}$ ». Rétrospectivement, Küss, rompu à la pratique de ces interventions, qualifie lui-même de «dramatiques et éprouvantes» les conditions dans lesquelles le prélèvement a été conduit à même le sol à la lumière d'une lampe ${ }^{351}$. L'inutilité thérapeutique de ces prélèvements, qui débouchent sur des transplantations marquées par des échecs rapides, participe sans doute à leur abandon dès 1951.

349 Praxis, 34, 1951, 741.

350 Idem.

351 Küss 1991, 39. 
En décembre 1952, Jean Hamburger et son équipe de l'hôpital Necker à Paris ouvrent la voie aux prélèvements sur donneurs vivants en acceptant de retirer un rein à la mère d'un jeune patient, Marius Renard, pris en charge en urgence dans leur service. Dès lors, moyennant le perfectionnement de connaissances dans le domaine de l'immunologie et le développement de procédés médicaux et chimiques pour maîtriser le rejet, ce type de donneur fournit la grande majorité des reins transplantés jusqu'au milieu des années 1960. Si le prélèvement d'un rein sur un individu sain représente une atteinte au précepte hippocratique primum non nocere, l'émergence de questionnements sur le bien-fondé de cette pratique est peu documentée dans les revues. Au sein de la communauté médicale, c'est plus la réalisation des transplantations en ellemême que la provenance de l'organe qui nourrit des discussions ${ }^{\mathbf{3 5 2}}$. Hamburger relate en effet, des années plus tard, dans une interview, les avis divergents qui existaient alors sur la valeur expérimentale de ces interventions:

"Je me souviens d'un collègue et ami anglais me disant que je n'avais pas le droit de tenter des greffes chez l'homme avant d'en avoir complètement compris les mécanismes. Pourtant, c'est de cette façon que progresse la science médicale, surtout s'il s'agit de maladies mortelles. Le praticien a alors le droit de tout tenter, en l'occurrence, la morale consiste à avoir toutes les audaces. Le cas des recherches sur les greffes illustre ce point de vue ${ }^{353}$.»

Dans notre corpus, ces expérimentations limitées à quelques équipes réparties entre différents hôpitaux de Paris et Boston, effectuées avec le consentement des donneurs, sont abordées dans une optique exclusivement scientifique. Dans Praxis, la notice qui relate en 1954 le prélèvement d'un rein maternel réalisé par Jean Hamburger évoque des "conditions exceptionnellement favorables ${ }^{354}$ ", mère et fils étant considérés, à l'aune des connaissances de l'époque, comme

352 Cameron 2002, 311.

353 Entretien avec Jean Hamburger, Jean-François Picard, 13 novembre 1990, www. histrecmed.fr/index. php?option $=$ com_content\&view $=$ article\&id $=53$ : hamburger jean\& catid=8:entretiens (consulté en 2019).

354 Praxis, 37, 1954, 794. 
"pratiquement homozygotes ${ }^{\mathbf{3 5 5}}$ » en vertu de leur proximité génétique. Si dans les revues médicales suisses l'événement est ainsi relayé par le biais de publications standards dans la revue de presse, le prélèvement d'un rein sur un donneur vivant soulève un intérêt hors de la sphère médicale. Dans le préambule d'une notice relative à une seconde publication proposée par l'équipe parisienne, le rédacteur P. Knobel rappelle qu'il s'agit de «ce cas de transplantation du rein d'une mère chez son fils, qui avait défrayé la chronique mondiale fin 1952, début $1953^{356}$ ", sans apporter d'autre précision, renvoyant à des faits connus de tous. Cette intervention est en effet relayée dans la presse quotidienne helvétique, notamment dans le Journal de Genève ou la Gazette de Lausanne, où plusieurs publications durant le mois de janvier 1953 évoquent par le biais de brèves notices l'évolution de l'état de santé du jeune patient jusqu'à son décès ${ }^{357}$.

Si l'atteinte physique portée au donneur est peu évoquée, tant par les spécialistes que dans les revues médicales ou dans la presse, c'est qu'elle est symboliquement diminuée par la valeur altruiste du don. Comme l'explique le médecin John S. Cameron dans son article sur l'évolution des pratiques cliniques et des considérations éthiques dans le cas du prélèvement sur donneur vivant, le don est envisagé comme une action simple dont l'unique motivation est de sauver un proche ${ }^{358}$. Le correspondant à Paris pour le Journal de Genève, James Donnadieu, l'illustre particulièrement dans l'article qu'il rédige après l'annonce du décès de Marius Renard. Il considère alors le don comme un témoignage "de l'amour d'une mère qui, après avoir donné la vie à son fils, la lui donnait une seconde fois ${ }^{359}$ ».

Sans envisager une redéfinition de la configuration des rapports interpersonnels entre receveurs et donneurs, le choix de ces derniers s'adapte tacitement aux contraintes biologiques qui le limitent au

\section{Idem.}

356 Praxis, 19, 1954, 415.

357 Journal de Genève (JdG), 19 janvier 1953, 8; JdG, 24 janvier 1953, 10; JdG, 28 janvier 1953, 10; JdG, 29 janvier 1953, 10; JdG, 31 janvier 1953, 5.

358 Cameron 2002, 312.

359 JdG, 31 janvier 1953, 5. 
cercle familial proche. Pourtant, le poids qui repose sur les donneurs qui constituent l'unique chance de survie pour le patient est parfois remis en cause au sein de la communauté médicale. C'est particulièrement le cas entre 1954 et 1958 lorsque les transplantations sont effectuées exclusivement entre jumeaux monozygotes. Alors qu'il préparait la première transplantation de ce type en 1954, John Murray, pionnier à Boston, mentionne en effet avoir été interpellé par des médecins qui s'y opposaient ${ }^{360}$.

Dans les revues médicales suisses, il faut attendre le milieu des années 1960 pour que le prélèvement sur donneur vivant suscite des questionnements au-delà des aspects strictement médicaux. Les résultats thérapeutiques encourageants, obtenus dès 1963 grâce à l'usage de stéroïdes dans le traitement du rejet, se traduisent par un intérêt accru de la communauté médicale pour la transplantation. On le constate par la tenue de plusieurs conférences durant lesquelles les pionniers évoquent la transplantation rénale devant un public médical profane en matière de transplantations. En 1964, Hamburger et le chirurgien Jean Vaysse, avec lequel le néphrologue collabore à l'hôpital Necker à Paris, présentent un rapport sur la transplantation rénale lors de la réunion annuelle de la Société suisse de médecine interne. Aux détails médico-techniques privilégiés dans les publications de résultats se substitue une description générale de la procédure médicale dont le prélèvement constitue le fondement. Dans le résumé qu'en propose $M \mho H$, Hamburger évoque «des problèmes d'éthique qu'il faut discuter. A-t-on le droit de prélever un rein chez une personne pour le greffer chez un malade? Il est incontestable que ce procédé apparaît contraire à l'éthique médicale traditionnelle ${ }^{361}[\ldots .$.$] . » Pour autoriser un prélèvement et minimiser$ symboliquement et médicalement l'atteinte portée à un individu en bonne santé, le néphrologue français fait appel au respect de deux conditions essentielles à ses yeux: «a) il faut être sûr de la qualité du volontariat (mère qui veut sauver son enfant) et suivant le donneur qui se propose il faut le soumettre à un examen psychologique pré-

360 Woodruff 1966, 17.

361 M\&H, 20 mai 1964, 469. 
alable afin que la décision soit prise en toute connaissance de cause et non pas dans un moment d'exaltation; b) les risques du donneur doivent être faibles par rapport aux chances du receveur, ce qui est bien le cas aujourd'hui: $0,05 \%$ de risque contre $50 \%$ de chances ${ }^{362}$.»

Façonnées par le contexte médico-technique et l'état des connaissances dans le domaine de l'immunologie, ces dispositions permettent la régulation de la pratique clinique en proposant un compromis entre besoin d'organes et protection du donneur. La force des liens affectifs entre donneur et receveur comme critère explicatif sur lequel évaluer le bien-fondé du don conduit les transplanteurs, au début des années 1960, à choisir indistinctement des donneurs apparentés et des donneurs non apparentés, mais affectivement proches. Les mauvais résultats obtenus lors de tentatives menées avec les reins de donneurs non apparentés marquent l'abandon tacite de prélèvement vivant en dehors de la fratrie ou des parents. Aussi, l'évaluation de la liberté dont dispose le donneur apparenté, unique chance de survie pour le receveur, continue de nourrir les discussions, en marge de la pratique, entre transplanteurs. Si les revues de notre corpus ne l'évoquent pas, Starzl estime lors du colloque Ciba de Londres en 1966 par exemple qu'il est difficile "d'imaginer comment quelqu'un ou un panel d'experts pourrait être certain que le don libre et sans coercition existe alors que le donneur potentiel est conscient qu'il prend une décision de vie ou de mort, et que sa décision est examinée ${ }^{363}$ ». Si un débat éthique concernant le prélèvement sur donneur vivant existe bel et bien, il s'avère quasiment absent dans les revues généralistes. Il est probable que ce type de questionnement reste cantonné aux revues spécialisées à cette époque.

La généralisation du recours aux organes cadavériques dans la seconde moitié des années 1960 marque, sous la plume de nombreux transplanteurs s'exprimant dans notre corpus, la résolution définitive des problèmes éthiques liés aux prélèvements humains. En 1971, dans un article relatant les activités conduites à Berne, le 
néphrologue André Montandon explique en effet que "grâce aussi à la possibilité d'utiliser des reins de cadavres, les hésitations de certains disparurent, le problème moral de prélever un organe à une personne saine ne se posant plus ${ }^{364}$ ».

Plus qu'un moyen complémentaire de fournir des organes, le prélèvement cadavérique constitue le prélèvement privilégié des programmes de transplantation rénale dans la plupart des hôpitaux européens ${ }^{365}$. Si les problèmes moraux liés à la sollicitation de donneurs vivants ont limité la mise en place de programmes, la possibilité de procéder à des échanges d'organes grâce au prélèvement cadavérique explique sans doute l'apparition de nombreux programmes dans les hôpitaux européens. Jusque-là, les difficultés techniques qui entourent l'identification cellulaire des donneurs constituent la principale entrave à l'extension de ce type de prélèvement. Pour les pionniers du prélèvement sur donneur vivant, comme Hamburger, le recours au rein de cadavres résout en effet les problèmes d'éthique médicale, mais entraîne la perte de "la moitié du progrès parcouru car on ne peut plus sélectionner les donneurs qui sont inconnus ${ }^{366}$ ».

Avec la mise en place de tests de typage cellulaire dès la fin des années 1960, la configuration d'échange centrée sur la recherche d'un donneur en fonction d'un receveur est remplacée par l'attribution d'un organe au receveur le plus compatible. Ce renversement, fondé sur l'identification des donneurs au sein des patients de l'hôpital et le regroupement des receveurs, constitue ainsi une adaptation de la pratique des transplantations à la structure centralisée hospitalière. Les bilans d'activité montrent que le recours au donneur vivant est limité aux cas particuliers de fortes compatibilités comme l'illustrent clairement les propos de René Mégevand à Genève relayés en 1974 : «De plus en plus, on a tendance à abandonner le donneur vivant apparenté au bénéfice du rein de cadavre, ne gardant comme exception que les situations "HLA identiques", c'est-à-dire au niveau de

364 RMSR, 6, 1973, 451.

365 Cameron 2002, 318.

366 M\&H, 20 mai 1964, 469. 
la fratrie ${ }^{367}$. " Ainsi, le prélèvement sur donneur vivant, limité aux cas pour lesquels les chances de succès sont les plus grandes, ne fait guère l'objet de questionnements éthiques.

\section{La définition de la mort cérébrale}

L'avènement de techniques de réanimation médicale permet, dès le début des années 1950, la suppléance de l'activité respiratoire et circulatoire chez des patients dont l'état physique détérioré en menace la régularité ${ }^{368}$. Si cette prise en charge est transitoire, certains patients, victimes d'une destruction définitive des fonctions cérébrales, ne reprennent jamais connaissance. Cet état décrit en 1959 dans la Revue neurologique par Maurice Goulon et Pierre Morallet sous le nom de "coma dépassé» se caractérise par l'irréversibilité des dommages qu'il cause au cerveau et par l'arrêt naturel des fonctions cardio-respiratoires en l'absence de mesures supplétives $^{369}$. Dans les revues médicales suisses, la notion de «coma dépassé » passe inaperçue au moment de son invention pour faire son apparition à la fin des années 1960, associée à la réalisation de transplantations rénales. Sous la plume de transplanteurs, le coma dépassé caractérise une catégorie de patients considérés comme des donneurs privilégiés dans des articles scientifiques ou comptes rendus de conférences destinés à des confrères non-spécialistes.

Lors d'un colloque concernant les activités menées à Zurich, en 1967, Largiadèr explique que les reins disponibles à l'hôpital cantonal proviennent "en règle générale de patients morts d'affection cérébrale $^{370}$ ». Si le corps médical s'accorde dans son ensemble à reconnaître l'irréversibilité de cet état, son assimilation à un état de mort clinique n'est pas unanime. Pour les transplanteurs zurichois, le diagnostic s'appuie sur des signes cliniques mesurables établis sous la forme d'une liste non standardisée. Immobilité totale des pupilles, absence de réflexe, pas de respiration spontanée cinq minutes après

367 Praxis, 63, 1974, 27.

368 Vachon 2010.

369 Hennette-Vauchet et Nowenstein 2009, 39-41.

370 Praxis, 22, 1967, 767. 
interruption de la respiration artificielle, baisse constante de la pression artérielle et électroencéphalogramme plat constituent ainsi un faisceau de preuves médicales de la destruction des fonctions cérébrales. Chez les médecins non spécialisés dans le domaine de la transplantation, comme pour les rédacteurs des notices, l'état de coma dépassé est envisagé comme un entre-deux auquel l'arrêt des mesures de réanimation vient mettre un terme. Dans le $B M S$, un médecin se demande «si cet état peut encore être qualifié de vie ou si - l'expression revient constamment - l'individu n'est plus qu'une préparation cœur/poumon ${ }^{371}$ ».

La mort cardiaque à laquelle conduit inévitablement le coma dépassé contribue à entretenir le flou, où ce dernier est envisagé parfois comme un pronostic de mort, parfois comme un diagnostic ${ }^{372}$. Aussi, la terminologie utilisée pour qualifier ce type de donneur potentiel varie sous la plume des différents médecins. Pour les transplanteurs de Zurich, où la mort est constatée avant le prélèvement, on parle de «Frischverstorbene ${ }^{373}$ ". À Genève, lors d'une séance de la société médicale en 1967, on le qualifie de "cadavre ou d'agonisant $^{374}$ ». Dans le BMS la même année, le rédacteur évoque "un mourant ${ }^{375}$ ». Ainsi, dans ce contexte où la mort est médicalement attestée alors que les fonctions respiratoires sont maintenues, les enjeux médicaux et légaux se concentrent autour de la détermination du moment qui autorise le prélèvement ${ }^{376}$.

Du côté des transplanteurs, la recherche d'une ischémie de durée minimale afin d'assurer un meilleur succès à la transplantation pousse à privilégier un prélèvement "à cœur battant » avant l'interruption de l'assistance circulatoire. Selon les critères en vigueur en Suisse pour définir la mort jusqu'en 1968, l'arrêt du cœur en constitue l'élément central. Sur cette base, prélever un organe avant

371 BMS, 10, 1968, 521.

372 Hennette-Vauchet et Nowenstein 2009, 45-46.

373 Praxis, 22, 1967, 767.

374 RSMR, 1, 1968, 50.

375 BMS, 20, 1968, 521.

376 Voir Bellanger, Steinbrecher et Tröhler 2003. 
l'arrêt respiratoire constitue une infraction susceptible de poursuites judiciaires. Prélèvement sur vivant en l'absence de consentement, atteinte corporelle entraînant la mort ou interruption volontaire des soins à prodiguer représentent autant d'infractions dont les médecins sont, aux yeux de la loi, responsables. Comme le bâtonnier de l'ordre des avocats du canton de Genève le rappelle lors d'une séance de la société médicale à laquelle il est convié, le coma dépassé ne bénéficie d'aucun statut spécifique, «la loi ne prévoit rien: elle connaît la vie et la mort ${ }^{377}$ ».

Aussi, les équipes médicales actives dans le domaine de la transplantation se trouvent confrontées à des problèmes pratiques que les propos d'un transplanteur rapportés dans le BMS illustrent:

«Doit-on, lorsqu'on décide de toute façon d'arrêter la circulation artificielle chez un mourant atteint de graves lésions au cerveau, opérer aussi longtemps qu'il y a circulation sanguine et interrompre seulement après la respiration artificielle, ou bien faut-il d'abord fermer l'appareil et enlever ensuite le rein ${ }^{378}$ ? »

Les protocoles opératoires qui régulent le prélèvement sur donneur cadavérique varient sans doute d'un centre à l'autre à cette période. Un des rares exemples relatifs à l'activité clinique des centres de transplantation dont nous disposons provient de l'hôpital de Zurich. Si pour l'équipe zurichoise, il est clair que la mort est «soit cardiaque, soit cérébrale ${ }^{379}$ ", les prélèvements sont effectués uniquement après la suspension des mesures de réanimation. Comme le précise Largiadèr, "[...] la constatation du moment exact de l'entrée dans la mort est pour des raisons morales et légales d'une signification déterminante ${ }^{380}$ ». L'écart entre mort médicalement incontestable et mort légale pousse cependant certains chirurgiens à pratiquer des prélèvements "à cœur battant» avant que la mort par arrêt circulatoire soit prononcée. À la fin des années 1960, le pionnier de la transplantation rénale en Belgique, Guy Alexandre,

377 RMSR, 1, 1968, 51.

378 BMS, 20, 1968, 521.

379 Praxis, 22, 1967, 768 [notre traduction].

380 Idem, 767 [notre traduction]. 
explique avoir assisté à un prélèvement mené avec l'accord d'un neurochirurgien pour lequel la mort du donneur potentiel était évidente «alors que le cœur battait encore ${ }^{381}$ ».

La transplantation cardiaque du Cap effectuée en décembre 1967 joue le rôle d'accélérateur dans la résolution des questions médicales et juridiques entourant l'identification du moment de la mort. L'urgence d'y trouver une réponse s'explique pour les médecins et les juristes par l'assimilation du prélèvement de cœur à la mise à mort du donneur. Comme l'explique Jean Graven, titulaire de la chaire de Droit pénal de l'université de Genève, lors d'une table ronde consacrée aux greffes cardiaques en juillet 1968, lorsqu' « on prend chez un "donneur" un cœur maintenu en état de survie par des moyens artificiels, on provoque par là même sa mort, on met intentionnellement fin à sa vie, on commet un homicide et même, puisqu'il y a préméditation marquée par les préparatifs opératoires, un homicide qualifié, ou assassinat (de même un "murder" ou homicide au premier degré dans le droit anglo-américain ${ }^{382}$ )».

Si chirurgicalement le prélèvement d'un cœur arrêté est réalisable, il conduit, de l'avis de plusieurs transplanteurs, à l'émergence de difficultés techniques supplémentaires. George Dureau de Lyon indique dans un article publié quelques semaines avant la greffe du Cap que «la clinique nous contraint à utiliser un cœur arrêté en anoxie [...]. Or ce cœur transplanté devra assurer immédiatement une reprise de fonction correcte et totale, car nous ne disposons pas de dispositif d'appoint, comme dans le cas du rein par exemple ${ }^{383}$.» Pour éviter la reprise fonctionnelle incertaine de l'organe, le prélèvement du cœur battant est privilégié.

Les questions médicales et légales entourant la définition du coma dépassé se déplacent ainsi sur la symbolique dont est chargé le cœur. Du côté des transplanteurs, les revues transmettent un avis unanime sur l'irréfutabilité de l'état de mort, même lorsque le cœur prélevé continue à battre grâce aux moyens artificiels. Pour

381 Alexandre 1991, 340 [notre traduction].

382 M\&H, 15 juillet 1968, 821.

383 M\&H, 7 juin 1967, 614. 
les juristes également, les médecins bénéficient d'une expertise incontestable sur laquelle fonder la définition de la mort, validant de fait les critères qu'ils décrivent. Lors d'une discussion rapportée dans les actes du symposium de la Ciba Foundation, Starzl évoque pourtant une réalité plus nuancée. Il explique en effet douter «[...] qu'aucun membre de notre équipe soit prêt à considérer la mort comme certaine aussi longtemps qu'on perçoit un battement de cœur ${ }^{\mathbf{3 8 4}}$ ». Qu'il s'agisse du personnel soignant, de médecins intensivistes ou des membres de l'équipe responsable du prélèvement, ces propos mettent en évidence l'existence d'incertitudes au sujet de la validité médicale de la mort cérébrale à la fin des années 1960 dans des centres au bénéfice d'une large expérience dans le domaine de la transplantation comme Denver. Ces doutes représentent une source d'étonnement suffisant pour que le rédacteur du BMS les mentionne dans le compte rendu du colloque qu'il publie ${ }^{385}$.

L'élaboration de directives relatives à la définition de critères de mort par des groupes d'experts, comme la Société allemande de chirurgie en avril 1968, le comité ad hoc de la faculté de médecine de Harvard relayé en septembre 1968, conduit à la résolution du vide médico-juridique qui entoure la pratique des transplantations «à cœur battant». Ces nouvelles normes permettent sinon la mise en place de programmes, du moins la réalisation d'expérimentations cliniques. La nécessité de se procurer des organes vascularisés pour assurer le plus de chances de succès aux transplantations se traduit donc à la fin des années 1960 par une adaptation du cadre légal. Comme dans le cas des prélèvements sur donneur vivant, les contraintes médico-techniques façonnent les questionnements éthiques dans les revues médicales généralistes.

\section{Justifier les expérimentations}

Si plusieurs tentatives de transplantations rénales sont attestées dès la fin des années 1930, elles se soldent par le rejet et l'absence de reprise fonctionnelle de l'organe. La plus retentissante est réalisée le

384 Murray 1966, 70 [notre traduction].

385 BMS, 20, 1968, 521. 
17 juin 1950 à Chicago par Richard Lawler, qui greffe un rein chez une patiente, en omettant de signaler à la communauté scientifique le parfait état de marche du second rein de sa patiente ${ }^{386}$. Si cette intervention met en évidence l'élaboration d'un savoir chirurgical par certains médecins, la connaissance du phénomène immunologique à l'origine du rejet s'établit moins rapidement. Construites sur les échecs qui marquent l'expérimentation animale et humaine dans le cadre des greffes de peau allogénique, ces tentatives suggèrent l'existence d'une identité propre à chaque receveur et à chaque donneur sans toutefois faire l'objet de description biologique précise ${ }^{\mathbf{3 8 7}}$.

Dans ce contexte médico-scientifique peu favorable, les «pionniers " expliquent le recours à la transplantation en raison de la défaillance physique irréversible des patients auxquels ils se trouvent confrontés. Dans le cas des interventions rénales, les notices dont nous disposons mettent en évidence une situation médicale atypique révélée soudainement et menaçant à court terme la vie du patient. Dans une notice, la revue $M \mho H H$ propose la description suivante sous le titre «la greffe d'un rein réalisée pour la première fois en France »:

«Il s'agissait d'une jeune malade souffrant d'hypertension artérielle grave que tout permettait de rattacher à une lésion rénale. Cette lésion rénale semblait être unilatérale, donc justiciable de l'ablation du rein malade. Or les examens cliniques, radiologiques et instrumentaux démontrèrent que parmi différentes malformations, sans doute, congénitales, la malade avait celle d'en avoir aussi une rénale; elle n'avait qu'un rein. Toute opération d'ablation était donc impossible ${ }^{388}$.»

Le décès des patients, entraîné par le rejet ou l'arrêt fonctionnel de l'organe, est considéré par les transplanteurs comme un échec que la contrepartie d'avoir tout tenté relativise. Lors d'une conférence devant la Société vaudoise de médecine en 1960, dont MひH publie les grandes lignes, Küss indique que "pour l'instant il est consolant de se dire que l'on n'a pas été néfaste à ses malades ${ }^{\mathbf{3 8 9}}$ ", le rédacteur

386 Kinneart 2006, 465.

387 Gentili 2015, 503.

388 M\&H, 15 février 1951, 70.

389 M\&H, 25 janvier 1961, 50. 
du compte rendu de conclure: "Savoir prendre ses risques est une devise de chirurgien ${ }^{390}$.»

Hors de la sphère médicale, ces tentatives bénéficient également de l'approbation de la presse, malgré leur caractère expérimental. À la suite du décès de Marius Renard, le patient greffé du rein maternel à Paris, le correspondant du Journal de Genève mentionne ainsi que pour lui «le sacrifice de Marius Renard n'aura pas été vain, car il aura certainement permis à la magnifique équipe de l'hôpital Necker de tirer des enseignements de ce drame ${ }^{391}$ ».

La formulation d'oppositions au déroulement de ces transplantations expérimentales est peu documentée dans les revues. Reflet sans doute du large assentiment dont elles jouissent, ces tentatives sont envisagées comme bénéfiques pour les receveurs en dépit des faibles chances de survie qu'elles offrent. Dans le cas de la transplantation cardiaque, on constate une prudence plus marquée dans le regard que posent les médecins et chirurgiens. Quelques mois après la transplantation de Barnard en 1967, alors que de nombreuses tentatives ont été effectuées à travers le monde, le chef du service de soins intensifs de Baden remet en cause, dans un courrier transmis au $B M S$, l'application clinique qu'il estime hâtive. Plus qu'uniquement lié aux chances de succès qu'il juge très faibles, c'est l'espoir que ce type d'intervention fait naître chez le patient qu'il réprouve. «Le malade à qui l'on tend la fragile planche de salut est-il en mesure de penser objectivement ${ }^{392}$ ? » écrit-il, en invoquant la responsabilité de chaque médecin confronté à ce type de situations. Même soumise à des critères fixés et évalués, la transplantation cardiaque est envisagée comme un «dilemme» par plusieurs chirurgiens dont $M \mho H$ se fait l'écho. Les échecs qui soldent ces interventions posent en effet la question de la planification de cette opération de dernier recours. Contrairement aux cas d'urgence dont font état les pionniers de la transplantation rénale au début des années 1950, la greffe cardiaque est réservée à des patients dont le passé médical est connu et est même

390 Idem.

391 JdG, 31 janvier 1953, 5.

392 BMS, 7, 1968, 157. 
proscrite comme traitement de la détérioration «subite» du cœur. Préconisée trop tôt, elle risque de raccourcir la vie du patient alors qu'effectuée trop tardivement, elle se révèle inutile, comme l'explique le cardiologue français Roger Froment dans MઐH: "nous devons donc prendre une décision chez les malades dont nous connaissons mal le degré de survie ${ }^{393}$.»

Comme les autres formes de transplantation en dehors du rein, la greffe cardiaque retrouve le statut d'intervention exceptionnelle, après avoir été l'objet de plus de 100 tentatives menées par 64 équipes dans 32 pays $^{394}$. Parallèlement à un travail de recherche scientifique en lien avec l'élaboration de protocole médicamenteux et d'affinement des critères de sélection et d'indication, elle est circonscrite à des centres spécialisés. Aux États-Unis, dès 1971, le National Heart Institute limite son soutien financier au centre de Standford, reconnaissant du même coup le statut d'expert incontesté à Shumway, son directeur.

Si la valeur thérapeutique de la transplantation ne fait aucun doute pour les médecins, c'est son application excessive sans expertise que révèle ce processus de repli. «Il est impensable qu'un esprit scientifique sérieux ne reconnaisse le bien-fondé de la transplantation quand elle permet le maintien à la vie, l'amélioration de sa qualité et le soulagement des souffrances qui portent gravement atteinte à l'existence ${ }^{395}$ ", estime à ce titre le chirurgien genevois Charles Hahn dans un des rares articles de notre corpus consacrés à la transplantation cardiaque durant les années 1970 .

\section{Le manque d'organes}

Le manque d'organes apparaît dans les revues dès les premières interventions menées dans les années 1950. Sous la plume des rédacteurs, il est considéré comme la conséquence inéluctable du développement de la transplantation rénale. Si le rejet et la conservation limitée des organes freinent l'application généralisée de ce type

$393 \mathrm{MEH}, 15$ juillet $1968,823$.

394 Hamilton 2012, 355.

395 M\&H, 25 janvier 1975, 133. 
d'intervention à de nombreuses pathologies, le statut provisoire de ces obstacles est indubitable pour les rédacteurs. Dans $M \mho H$, l'auteur s'interroge: "Les affections du rein et l'hypertension artérielle ont-elles désormais trouvé dans la greffe de rein sain des chances de guérison? Si c'est le cas, où trouvera-t-on les reins sains nécessaires à tant d'hypertendus et de néphrétiques ${ }^{396}$ ? »

Avec l'omniprésence du prélèvement sur donneur vivant jusqu'au milieu des années 1960, la transplantation est orientée vers un choix limité de donneurs compatibles, à savoir les jumeaux homozygotes ou les membres d'une fratrie. Fantasmé lors des premières expérimentations, le manque d'organes se transforme dans les revues en manque d'organes adéquats, causé par l'incompatibilité d'organes prélevés hors du cercle familial. Sans pouvoir être infléchi par un volontariat du don plus marqué, il est abordé en lien avec la présentation de travaux consacrés à la recherche de types de prélèvements alternatifs. À cet égard, le prélèvement d'organes d'origine animale apparaît sous la plume de certains chercheurs, au même titre que le prélèvement cadavérique, comme un moyen à envisager pour résoudre le manque de greffons ${ }^{397}$.

Avec la généralisation du prélèvement cadavérique, l'organe disponible est attribué au receveur bénéficiant de la compatibilité cellulaire la plus marquée. Un décalage apparaît dans les hôpitaux, où les receveurs sont rassemblés par liste, entre les ressources disponibles et les receveurs en attente. Sous la plume des transplanteurs, c'est le manque de donneurs qui conduit à la disponibilité insuffisante d'organes compatibles. "En raison du manque de donneurs de reins, le choix du receveur est une décision difficile. Elle sera encore plus difficile lorsque la transplantation rencontrera un plus grand succès et que les indications seront élargies ${ }^{398}$ ", avertit le chef du service de chirurgie de Zurich, Åke Senning, en 1967. La création de réseaux d'échanges basés sur le partage d'informations entre les établissements constitue un moyen d'optimiser l'utilisation des

396 M\&H, 15 février 1951, 70.

397 M\&H, 25 mars 1964, 251.

398 Praxis, 22, 1967, 767 [notre traduction]. 
organes prélevés. Le néphrologue André Montandon explique en effet en 1973 qu'à l'hôpital de Berne "plus de la moitié des organes ainsi mis à notre disposition ne peuvent être utilisés sur place ${ }^{399}$ ". Malgré les échanges effectués entre les différentes institutions, la disponibilité d'organes compatibles est insuffisante pour couvrir le nombre de receveurs en attente. Le néphrologue bernois y voit la conséquence «d'un nombre de donneurs bien trop faible par rapport à celui des malades figurant sur la liste d'attente ${ }^{400}$ ».

La plupart des médecins, dont les revues de notre corpus se font l'écho, envisagent l'information du public comme le moyen privilégié pour faire accepter la transplantation et entraîner, par conséquent, l'augmentation du nombre de donneurs. "L'exemple de la greffe cornéenne a montré quel succès cette information peut avoir, puisque l'opposition du début a été en grande partie éliminée et qu'il n'y a plus de manque de matériel ${ }^{401}$ ", explique un rédacteur du BMS. La certitude d'une corrélation entre l'adhésion du public à la transplantation et la hausse de nombre de donneurs est fortement marquée dans l'esprit des transplanteurs de cette période. Aussi, la modification des procédures juridiques régulant l'obtention d'organes est peu discutée dans les revues à la fin des années 1960 et durant les années 1970. En effet, le consentement explicite du donneur ou de sa famille constitue un prérequis sinon juridique, au moins "éthique ", au prélèvement que peu de transplanteurs et de profanes remettent en cause au début des années 1970. Alors que le Tribunal fédéral valide la légalité du prélèvement effectué selon le modèle du consentement présumé à Zurich lors de la greffe cardiaque d'avril 1969, le directeur de l'hôpital de Berne préfère continuer à solliciter systématiquement l'accord de la famille ${ }^{402}$. «Nous avons toujours demandé l'assentiment de la famille avant de

399 RMSR, 6, 1973, 453.

400 Idem.

401 BMS, 20, 1968, 520.

402 Staatsarchiv Bern, Insel, Abteilungstransplantation, die Organtransplantation am Inselspital im Lichte der neuesten Bundesgerichtspraxis, p. 9. 
prélever les reins ${ }^{403}$ ", précise d'ailleurs Montandon. Dans certains pays, comme l'Angleterre, la reconnaissance juridique du modèle de consentement présumé est toutefois envisagée comme un moyen de combler le manque d'organes, faute de pouvoir influencer la position du public. Un rapport présenté à la Chambre des communes en 1968 indique en effet qu'il «est impossible en dépit des moyens mis en œuvre de convaincre suffisamment d'adeptes et de volontaires pour pouvoir disposer d'une réserve appropriée d'organes destinés à la transplantation ${ }^{404}$ ».

À travers les propos relatés par les revues, les transplanteurs semblent penser qu'un changement d'attitude de la population à propos de la transplantation n'est qu'une question de temps. Cette confiance s'établit sur la comparaison avec d'autres pratiques, d'abord contestées avant d'être adoptées par le public. «Rappelezvous le même genre de controverses qui est né au début des transfusions sanguines, mais aujourd'hui, elles sont acceptées sans discussion par tout le monde ${ }^{405}$ ", mentionne Denton Cooley dans une interview réalisée avec Rentchnick.

Si les moyens concrets à mettre en place pour informer le public restent mal définis au début des années 1970, ils se concentrent progressivement sur le rôle central dont est investi le médecin généraliste. Pivot entre le public et les transplanteurs, les praticiens doivent être unis derrière ce qui est désigné comme une "mission" dans un article d'information sur la transplantation, publié en 1979 dans le $B M S$ : «Le besoin d'organes pour les transplantations n'est pas couvert, il augmente. L'atténuation de la pénurie d'organes est une mission commune à l'ensemble du corps médical ${ }^{406}$. »

Dès la fin des années 1970, le manque d'organes est également présenté comme le résultat d'un nombre trop faible de prélèvements effectués ${ }^{407}$. La motivation défaillante des équipes de réanimation,

403 RMSR, 6, 1973, 453.

404 M\&H, 11 décembre 1968, 1481.

405 M\&H, 18 septembre 1968, 1032.

406 BMS, 16, 1979, 1232 [notre traduction].

407 RMSR, 100, 1980, 710. 
indirectement concernées par la transplantation, surtout dans les hôpitaux périphériques dépourvus de programme de transplantation, est invoquée par les médecins. Le plus souvent envisagé comme un phénomène factuel dans les bilans d'activité, le manque d'organes devient peu à peu personnifié par le malade condamné à mourir. Dans un article de 1975 consacré aux recherches menées au King's College Hospital sur les maladies du foie, B. Evans, correspondant à Londres pour $M \mho H H$, explique en effet que "comme dans le cas d'une autre mesure destinée à sauver la vie, la greffe de rein, le manque de donneurs restreint beaucoup les possibilités pour beaucoup de malades du foie et beaucoup d'entre eux meurent avant qu'on ait trouvé un foie qui pourrait convenir à la greffe ${ }^{408}$ ».

Le manque d'organes incarne progressivement la cause du décès reléguant en arrière-plan la défaillance fonctionnelle de l'organe.

\subsection{Conclusion}

Les considérations "éthiques"sont peu rapportées dans les revues de notre corpus aussi bien par les transplanteurs que par les médecins non-spécialistes. Le développement des transplantations se constitue entre les années 1950 et la fin des années 1970 face aux usages et procédures mis en place par les médecins spécialistes pour se procurer des organes. La régulation des pratiques et des questionnements "éthiques" qui en découlent s'effectue au sein de la sphère médicale sous forme de compromis qui permettent la poursuite des investigations médico-scientifiques, malgré les échecs obtenus lors des phases d'émergence.

Lors des interventions menées dans le cadre du développement de la transplantation rénale dès les années 1950, les facteurs biologiques et techniques orientent le choix des types de donneurs privilégiés. Bien que la liberté de choix restreinte dont il bénéficie soit reconnue, le donneur vivant apparenté représente l'unique type de donneur dans le cas de la transplantation rénale jusqu'au début des années 1960. Ce sont la meilleure gestion du phénomène 
biologique du rejet et l'acquisition de connaissances plus précises dans le domaine de l'immunologie qui permettent son remplacement au profit du prélèvement cadavérique. Malgré la réprobation dont le prélèvement sur donneur vivant fait l'objet dans la plupart des centres au début des années 1970, il reste préconisé dans le cas de compatibilité exacte des jumeaux monozygotes. C'est le succès assuré par l'identité cellulaire identique qui en autorise le recours.

La coexistence de travaux de recherche au début des années 1960 dans les domaines de la xénotransplantation et du prélèvement cadavérique montre également que durant cette période, les sources d'organes potentiellement transplantables échappent à une désignation préalable élaborée en raison de motifs "éthiques". Les difficultés médicales insurmontables que rencontrent les chercheurs mènent à l'abandon de la xénotransplantation. À cet égard, on peut se demander quel serait aujourd'hui le positionnement général au sein de la sphère médicale et de la population face à la transplantation animale si son usage permettait de sauver des vies humaines.

C'est dans l'optique de bénéficier d'organes sans avoir à recourir aux donneurs vivants, mais aussi par la disponibilité de donneurs cadavériques à l'hôpital, sur place, qu'émerge la question de la définition de la mort cérébrale. Jusqu'à l'opération du 4 décembre 1967 au Cap qui donne une notoriété décisive à la transplantation, le cas du coma dépassé constitue une réalité médicale à laquelle sont déjà confrontés certains médecins et qui permet la réalisation de transplantations rénales, sans toutefois faire l'objet d'une définition de la part d'institutions reconnues. Aussi, il semble légitime de s'interroger sur l'influence de la médiatisation de la transplantation cardiaque sur l'élaboration de directives et prescriptions médicojuridiques. Hors du cadre de son application à la transplantation cardiaque, le «coma dépassé » aurait-il fait l'objet de réglementation aussi rapidement?

La maîtrise chirurgicale représente une justification tacite pour recourir à la transplantation. Pour bénéficier d'une reconnaissance thérapeutique au sein du corps médical, elle est limitée toutefois à un nombre restreint d'experts, en marge de la pratique thérapeutique habituelle, appliquée dans le cadre de situations cliniques définies 
par des critères mesurables. La vague d'engouement qui entoure la transplantation cardiaque à la fin des années 1960 constitue ainsi une rupture qui explique l'apparition plus marquée de considérations éthiques aussi bien au sein du corps médical que dans la sphère publique.

À travers l'adaptation des prélèvements à la structure hospitalière, le manque d'organes, sur lequel l'emprise des médecins est faible jusque-là, se transforme dans le discours médical en manque de donneurs. Il est dès lors envisagé comme dû à des facteurs divers tels que le refus de donner ou le manque de motivation des équipes chargées de l'identification de donneurs potentiels.

Comme à ses débuts, la transplantation reste aujourd'hui marquée par la problématique de l'obtention d'organes. D'autres formes de prélèvements, comme ceux effectués sur donneur «à cœur arrêté ", montrent que la pratique s'étend, soulevant de nouveaux questionnements. Certains modes d'obtention restent cependant largement proscrits en raison de leur adaptation impossible, sous forme de compromis, à la pratique de la médecine telle qu'elle est envisagée à ce jour au sein de la sphère médicale. La rétribution financière du don en constitue sans doute l'exemple le plus frappant hier comme aujourd'hui. 



\section{Chapitre 5}

\section{Le don et la transplantation d'organes dans la presse quotidienne: du problème biologique au problème social}

Raphaël Hammer

L'accession d'une question ou d'une situation au rang de problème public bénéficie souvent du concours, volontaire ou involontaire, des médias qui constituent un puissant acteur de mise en visibilité ${ }^{409}$. Les textes médiatiques sont ainsi un révélateur important des discours et images qui circulent dans l'espace social sur un objet. L'un des objectifs de la recherche consistait à comprendre comment les représentations publiques du don et de la transplantation d'organes se sont transformées dans la presse quotidienne. En particulier, il s'agissait d'examiner le passage progressif de la transplantation comme technique médicale expérimentale à la fin de la Seconde Guerre mondiale au don d'organes, comme problématique de santé publique et comme problème public. Autrement dit, suivre comment, en l'espace de quelques décennies, la transplantation humaine a émergé et s'est développée comme domaine d'intérêt de la sphère médiatique dans le contexte suisse.

409 Neveu 2015b. 
Cette évolution tient évidemment en large partie aux progrès scientifiques et techniques mais aussi aux développements institutionnels et éthiques qui dessinent et organisent les modalités de l'échange d'organes dans la société. L'inscription de la transplantation dans le corps social, dans les mentalités populaires, suppose aussi le déploiement de discours publics qui contribuent à forger une ou des images de ce qu'est l'échange d'organes d'un individu à l'autre, à lui associer des valeurs, des références positives ou négatives. La médiatisation donne accès dans une certaine mesure aux discours qui sont produits dans la société sur un sujet donné.

Dans ce chapitre, nous examinerons la couverture médiatique de la transplantation d'organes sur un plan quantitatif (quelle place occupe ce sujet dans la presse? Y a-t-il des périodes de forte et faible médiatisation, et comment peut-on l'expliquer ?) et qualitatif (quels thèmes de la transplantation sont abordés et dans quels termes? Quels sont les arguments mobilisés? Que révèlent-ils du contexte normatif ambiant ?). L'étendue de la période prise en compte (environ un demi-siècle) permet d'aborder ces deux objectifs dans une perspective diachronique en tenant compte des spécificités historiques du développement de la transplantation sur les plans scientifique et institutionnel notamment.

Nous avons constitué un corpus de presse composé d'articles issus du Journal de Genève ( $J d G$ ) couvrant la période du $1^{\text {er }}$ janvier 1945 au 28 février 1998. Fondé en 1826, le JdG fut le principal quotidien d'information de référence en Suisse romande et d'audience internationale jusqu'à sa disparition à la fin des années $1990^{410}$. Il est en outre l'un des rares quotidiens helvétiques à offrir une telle continuité historique.

Pour prendre la mesure de l'évolution de la médiatisation de la transplantation d'organes dans ses différentes dimensions, nous avons fait le choix de critères d'inclusion assez large des articles. Si l'analyse porte principalement sur la transplantation et le don d'organes solides (d'un donneur vivant ou décédé), nous avons

410 Le dernier numéro du Journal de Genève (devenu en 1991 le Journal de Genève et gazette de Lausanne) date du 28 février 1998. Il fusionne alors avec Le Nouveau Quotidien pour donner lieu au journal Le Temps. 
aussi inclus les articles évoquant les organes artificiels, les transplantations d'organes chez l'animal (pour autant que l'horizon de la transplantation chez l'homme figure d'une manière ou d'une autre), les greffes de moelle, de neurones ou encore de gènes, ainsi que les greffes de membre. Nous avons retenu les articles dans lesquels la transplantation et/ou le don d'organes constituent le sujet principal de l'article, ainsi que ceux dans lesquels ils sont un thème secondaire, c'est-à-dire abordés dans une partie seulement de l'article. En revanche, les articles dans lesquels notre sujet d'intérêt apparaissait de manière très marginale ont été exclus du corpus. Le genre journalistique n'a pas constitué un critère de sélection - seuls les lettres de lecteurs et les comptes rendus de livres ou de films ont été exclus. Au total, le corpus regroupe trois cent quatre-vingt-six articles de longueur variable ${ }^{411}$.

Dans un premier temps, nous décrirons les tendances générales de la médiatisation de la transplantation et du don d'organes entre 1945 et 1998. Dans un deuxième temps, nous nous centrerons sur les principaux enjeux éthiques présents dans le corpus. Ces enjeux concernent les questions «morales» ou "philosophiques» que posent notamment les premières transplantations cardiaques, telles que l'intégrité du corps, leur justification médicale ou encore le consentement. Dans un troisième temps, nous examinerons la question de la pénurie d'organes qui est particulièrement révélatrice du processus de "socialisation" de la transplantation puisqu'il transforme un problème médical (le manque de greffons) en une question sociale (le manque de donneurs) ${ }^{\mathbf{4 1 2}}$.

411 Le corpus est constitué de $25 \%$ de "brèves" (textes d'une dizaine de lignes environ, en principe dépourvus de titre) et de $19 \%$ de "filets" (textes d'une vingtaine de lignes comportant un titre), le $56 \%$ restants étant des articles d'une longueur supérieure (jusqu'à une page). Dans le corps du texte, sauf exceptions, nous utiliserons le terme "article» au sens générique (sans distinction de longueur).

412 Rappelons que les discours médiatiques ne sont évidemment jamais le pur reflet de l'actualité, qu'elle soit politique, scientifique, juridique ou scientifique (Charaudeau 1997). Tout événement d'actualité relaté par un quotidien résulte d'un choix rédactionnel évaluant s'il vaut la peine d'être relayé au public (ces raisons pouvant être purement commerciales). En outre, l'information d'un événement fait l'objet d'une mise en forme et en contenu plus ou moins importante de la part du journaliste. Enfin, l'information contenue dans un quotidien peut ne pas être motivée par l'actualité mais refléter une activité rédactionnelle propre, sous la forme de reportages ou d'enquêtes. 


\subsection{La médiatisation de la transplantation entre 1945 et 1998}

Dans cette première section, nous décrivons l'évolution chronologique et thématique de la couverture médiatique de la transplantation d'organes, pour détailler ensuite le contenu des articles en nous centrant sur les deux périodes caractérisées par une médiatisation significative du don et de la transplantation d'organes.

\section{Tendances chronologiques et thématiques de la couverture médiatique}

La répartition par année de l'ensemble du corpus permet de brosser à grands traits l'évolution sur un demi-siècle de la couverture médiatique. Le Graphique 1 conduit schématiquement à observer quatre périodes principales ${ }^{413}$ :

1) une médiatisation très faible, voire inexistante, entre 1945 et 1966; cette période regroupe 34 articles (9\% du corpus) et se caractérise par l'absence totale de mention du thème de la transplantation d'organes dans environ une année sur deux;

2) un pic très marqué de médiatisation du thème de la transplantation entre 1967 et 1969 ; ces trois années totalisent plus d'un tiers des articles du corpus $(\mathrm{n}=133 ; 35 \%)$, ce qui correspond à une moyenne de 44 articles pour chacune de ces années;

3 ce pic est suivi par un fort tassement de la médiatisation de 1970 à 1981 avec moins de 4 articles en moyenne par année ( $\mathrm{n}=45 ; 12 \%)$;

4) enfin, la période 1982-1997 est caractérisée par une médiatisation modérée et relativement stable ( $\mathrm{n}=171 ; 44 \%)$, avec un peu plus de 10 articles en moyenne par année.

Si l'on peut ainsi constater de fortes variations dans la couverture médiatique au fil du temps, il convient d'établir dans quelle mesure l'évolution chronologique de la médiatisation reflète des variations du point de vue du contenu des articles ${ }^{414}$. À cet effet, nous avons classé

413 Bien que représentée dans le graphique, l'année 1998 n'a pas été prise en compte dans les calculs du fait qu'elle n'est couverte que partiellement. Le total de référence est donc ici de 383.

414 Bardin 1996. 


\section{Graphique 1}

\section{Nombre d'articles consacrés par année à la transplantation et au don d'organes $(n=386)$}

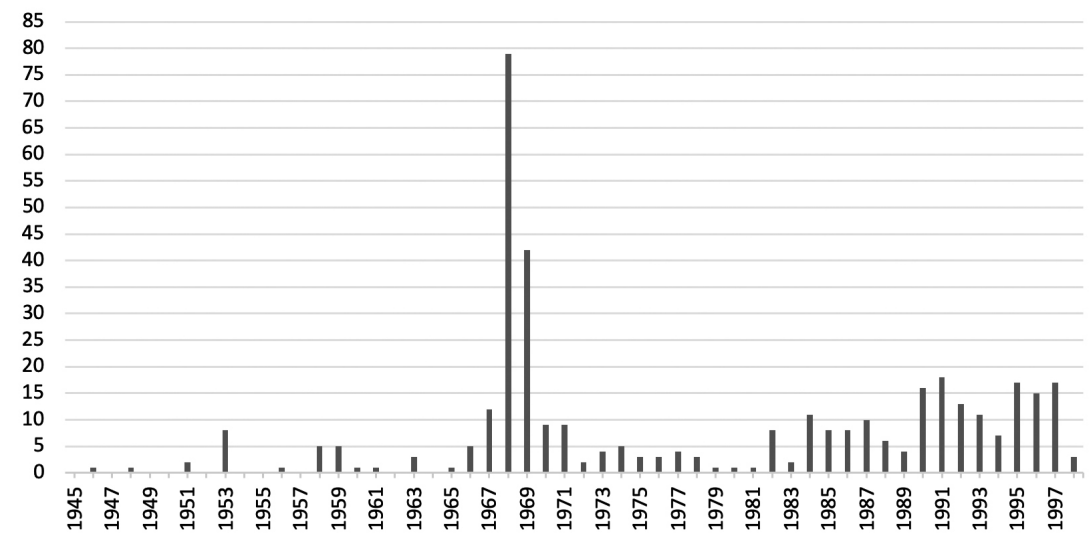

l'ensemble du corpus selon quatre catégories élaborées de manière inductive qui décrivent le thème principal selon lequel la transplantation et/ou le don d'organes sont traités dans chaque article.

Premièrement, la catégorie "science et médecine " inclut les articles qui abordent la transplantation principalement sous ses aspects scientifiques, techniques, médicaux et biologiques. Cette catégorie est largement dominante puisqu'elle s'applique à près des deux tiers du corpus $(\mathrm{n}=251$ articles; $65 \%)$. Larticle typique consiste en une nouvelle qui informe le lecteur d'une "première" chirurgicale, en décrivant son déroulement et l'état du patient transplanté. Les articles de cette catégorie "science et médecine» rendent également compte de découvertes ou d'avancées dans différents domaines de la médecine (immunologie, pharmacologie, chirurgie...), et certains dressent un bilan des connaissances et de l'activité de la médecine de transplantation pour tel ou tel organe.

Deuxièmement, nous avons distingué une catégorie "éthique et société » ( $\mathrm{n}=54 ; 14 \%$ du corpus) qui regroupe les articles abordant la transplantation et le don d'organes d'une part sous des aspects éthiques $(n=41)$, et d'autre part d'un point de vue sociétal au sens large (psychosocial, culturel, philosophique et spirituel ou religieux notamment) $(\mathrm{n}=13)$. Cette catégorie inclut des articles adoptant 
parfois une approche critique, ainsi que des articles qui soulèvent des questionnements ou qui évoquent des aspects problématiques en lien avec la transplantation et le don en termes d'opinion publique, de croyances, de valeurs ou de légitimité par exemple.

Troisièmement, les articles appartenant à la catégorie «régulation institutionnelle» $(\mathrm{n}=43 ; 11 \%$ du corpus) traitent de la définition des activités de transplantation et du don d'organes sur les plans politique, économique, juridique et hospitalier. Ces articles concernent avant tout la régulation politico-législative du don et de la transplantation d'organes ( $\mathrm{n}=27)$, et, dans une moindre mesure, des aspects économiques et juridiques $(\mathrm{n}=8)$, et touchant à l'organisation du système de santé et des pratiques hospitalières $(\mathrm{n}=8)$.

Quatrièmement, la catégorie "pénurie d'organes" regroupe les articles centrés sur la problématique du besoin ou du manque d'organes, et qui abordent les causes du manque d'organes, ses conséquences ainsi que les mesures visant à résoudre ce qui est devenu progressivement un problème de santé publique $(\mathrm{n}=28$; $7 \%$ du corpus) $)^{415}$.

La répartition chronologique de ces quatre catégories thématiques permet de voir si ces différentes manières d'aborder la transplantation d'organes sont privilégiées à certains moments plutôt qu'à d'autres. Afin de faciliter la démarche de comparaison, le corpus a été ventilé par décennie ${ }^{416}$. Le Graphique 2 montre ainsi que le cadrage "science et médecine» prédomine fortement quelle que soit la décennie considérée. Cette prépondérance diminue toutefois, en termes proportionnels, au cours du temps: $90 \%$ dans les années 1950, $85 \%$ dans les années 1960, 79\% dans les années $1970,70 \%$ dans les années 1980 , et $33 \%$ pour la période 1990-début 1998. Cette baisse relative du cadrage médico-scientifique témoigne surtout du fait que le thème de la transplantation est progressivement associé à des enjeux diversifiés. On le voit à l'examen des catégories "régulation institutionnelle» et "pénurie

415 Une cinquième catégorie "divers" ( $n=10 ; 2,6 \%$ du corpus), englobant les articles n'entrant dans aucune des catégories précédentes, a été exclue des analyses qui suivent.

416 À l'exception des années 1945-1949 et 1990-1998. 
d'organes" notamment qui font leur apparition dans le $J d G$ dans les années 1980 pour occuper une part substantielle des articles parus dans les années 1990 (respectivement $27 \%$ et 19\%). Quant à la catégorie "éthique et société ", elle émerge dans les années 1960 (15 articles), diminue dans les deux décennies suivantes (5 articles dans les années 1970, 9 dans les années 1980) et connaît un rebond important dans les années 1990 (23 articles). Il convient cependant d'être prudent dans l'interprétation de ces tendances dans la mesure où elles sont fondées sur le cadrage dominant des articles. Cela signifie que des considérations d'ordre éthique ou en lien avec la pénurie d'organes, par exemple, existent dans des articles centrés sur les aspects scientifiques ou sur la régulation institutionnelle de la transplantation. Ces tendances livrent donc une image partielle du contenu des articles du corpus et de son évolution.

\section{Graphique 2}

\section{Distribution des catégories thématiques par décennie $(n=376)$}

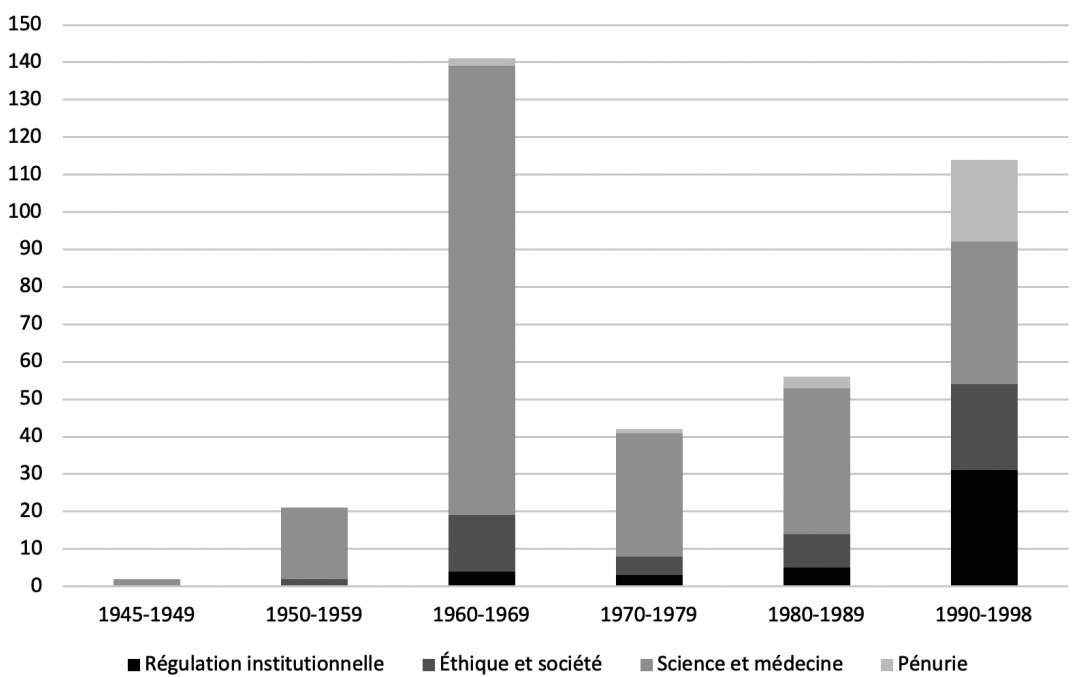

Il convient à présent d'analyser de plus près la médiatisation de la transplantation et du don d'organes en nous centrant sur les deux périodes phares identifiées ci-dessus, à savoir la fin des années 1960 et les décennies 1980 et 1990. À cet effet, nous décrivons les princi- 
paux événements et informations qui nourrissent ces deux périodes, ainsi que la nature des enjeux qui émergent des articles ${ }^{417}$.

\section{Les transplantations en plein essor (1967-1969): espoirs et prudence}

Le pic médiatique des années 1967 à 1969 relève presque exclusivement d'articles de type médico-scientifique $(\mathrm{n}=110 ; 84 \%$ des 131 articles couverts par ces trois années). Ce pic tient d'abord aux exploits chirurgicaux du médecin sud-africain Christiaan Barnard. Si la première transplantation cardiaque considérée comme réussie, qu'il a réalisée en décembre 1967 au Cap, a fait l'objet de plusieurs articles $(\mathrm{n}=5)$, la deuxième transplantation cardiaque à son actif a bénéficié d'une couverture médiatique bien plus importante ( $\mathrm{n}=16)$, du fait que Philip Blaiberg - le patient de la deuxième transplantation - a survécu bien plus longtemps à son opération que Louis Washkansky (neuf mois contre 18 jours). Le nombre très élevé d'articles consacrés à la transplantation d'organes à la fin des années 1960 tient ensuite au boom des transplantations tentées à travers le monde à la suite de Barnard. Ce sont quelque cinquante opérations de transplantation, du cœur principalement, dont le lecteur du $J d G$ aura pu prendre connaissance de décembre 1967 à fin $1969^{418}$. Il est ainsi fait mention, généralement sous forme de brèves ou de filets, de la première transplantation du cœur réalisée par exemple à New York, Stanford, Bombay, Paris, Houston, Montpellier, Dallas, Madrid, Caracas, Lyon, Marseille, Turin, Munich et Zurich. Ces deux années pour le moins prolifiques font également état d'autres opérations spectaculaires: transplantations sur des enfants et des bébés, transplantation de pancréas, de larynx, d'intestins, ou encore prélèvement simultané de six organes.

417 Notons que la période des années 1950 jusqu'au milieu des années 1960 se caractérise par plusieurs articles de fond consacrés aux aspects biologiques et expérimentaux de la greffe, dans lesquels les expérimentations menées chez les animaux occupent une place importante. Sur le plan factuel, ce sont notamment les greffes de moelle dans l'épisode des "atomisés " yougoslaves (en 1958) qui retiennent l'attention, ainsi que les tentatives de transplantations rénales.

418 Par comparaison, seule une dizaine de transplantations en tout genre sont relatées entre 1970 et 1981. 
Bon nombre des articles du pic de médiatisation de la fin des années 1960 prennent la forme d'un bulletin médical livrant des informations sur l'organe transplanté, le nom du chirurgien, le lieu du déroulement de l'opération, ainsi que l'évaluation du succès médical de la transplantation et de l'état de santé du patient. La mention du nom et prénom du receveur et du donneur, ainsi que l'évocation des circonstances médicales du décès du donneur sont fréquentes. Le compte rendu des transplantations de plusieurs patients fait l'objet d'un traitement médiatique particulier sous la forme d'une «chronique des opérés". Une série de plusieurs articles espacés dans le temps sont en effet consacrés à ces transplantations, dont le point de départ est l'exposé de l'opération elle-même, suivi d'un ou plusieurs bulletins de santé du patient quelques jours, semaines, ou mois après l'opération. Ces «chroniques des opérés" prennent fin généralement avec la nouvelle du décès du receveur. Le cas de Blaiberg en est la meilleure illustration. Outre plusieurs articles successifs qui informent le lecteur de l'évolution de l'état de santé du patient, de son état d'esprit et de ses progrès de convalescence, un article détaille le déroulement médical de l'opération, un autre rapporte ses déclarations lors d'une interview, un autre rend compte du fait que "Philip Blaiberg a tenu son propre cœur dans ses mains ${ }^{419}$ ", un autre relate son renoncement à subir une nouvelle opération, et enfin un dernier article informe de son décès.

Les transplantations du cœur en particulier donnent lieu à une comptabilité précise, comme l'illustre une brève du 22 octobre 1968 qui relate «la septième transplantation du cœur ${ }^{420}$ » au Canada. Elle précise qu'il "s'agit du soixante-quatrième patient auquel une greffe du cœur a été opérée depuis décembre dernier». Le décompte du nombre de survivants et l'identification nominale du «doyen » de tel ou tel type de transplantation font assez rapidement leur apparition. Le 24 juin 1968, une brève annonçant la mort du premier transplanté cardiaque au Brésil établit un bilan de la manière suivante: "il ne reste plus que quatre hommes vivant avec un cœur

41919 février 1968, 10.

42022 octobre, 3. 
greffé, M. Philipp Blaiberg, le révérend père Boulogne et les deux opérés de Houston, Everett Thomas et Luis Fierro ${ }^{421}$.» Le 18 août 1969, l'annonce du décès de Blaiberg bouscule le classement et ce changement de «doyen des survivants de greffes cardiaques» fait à lui tout seul l'objet d'un filet: "Philip Blaiberg a transmis sans le vouloir, au révérend père Boulogne, le redoutable honneur d'être le plus ancien survivant de la greffe de cœur ${ }^{422}$.»

Le «recensement» personnalisé des transplantés, la rhétorique des «premières" médicales ainsi que la comptabilité des records de survie traduisent une fascination vis-à-vis des exploits réalisés en même temps que les promesses d'un nouveau pan de l'histoire de la médecine. L'information prolifique sur les multiples tentatives de transplantations, notamment cardiaques, reflète l'espoir d'une révolution thérapeutique tout en affichant une certaine prudence. Par exemple, dans le compte rendu d'une transplantation rénale tentée en Angleterre en 1963, il est précisé que les responsables de l'opération "ont grand soin surtout de mettre les médecins et le public en garde contre tout optimisme prématurée ${ }^{423}$ ». La pondération est particulièrement de mise s'agissant de la transplantation du cœur: «on voit que les expériences récentes de greffe cardiaque ne sont malheureusement pas près de modifier l'évolution d'une des principales maladies modernes de l'humanité ", et que même en cas de succès, les transplantations «ne représentent pas la solution-miracle aux détériorations organiques $^{\mathbf{4 2 4}}$ ». Si pour Barnard les transplantations de cœur humain constituent une étape vers les implantations de cœurs artificiels et d'origine animale, elles n'en constituent pas moins une technique éprouvée; «la transplantation d'organes a un avenir remarquable [...] et les découvertes en cours sont très prometteuses ${ }^{425}$ ». Si à la fin des années 1960 la transplantation cardiaque suscite de l'optimisme et si elle est largement célébrée comme un événement historique, elle fait

42124 juin 1968, 5.

42218 août 1969, 14 .

42320 février 1963, 1 .

42424 mai 1968, 15.

42517 octobre 1968, 18. 
aussi l'objet d'attitudes plus réservées et d'avis divergents au sein du corps médical dont plusieurs articles rendent compte.

Les discours mitigés reposent parfois sur des arguments de coût et sur des considérations morales ou philosophiques, mais principalement sur l'obstacle que constitue le rejet dont les mécanismes sont loin d'être maîtrisés, ce qui est alors communément admis dans la communauté scientifique. L'article annonçant la première transplantation cardiaque de Barnard accorde une large place au scepticisme de plusieurs médecins allemands quant au succès de l'opération "parce que le problème causé par la défense du corps contre les tissus étrangers n'est pas encore résolu, de même que la question de la conservation du

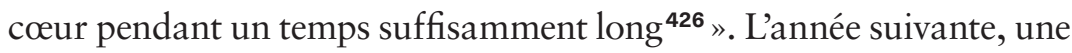
journaliste couvre un congrès d'angiologie et rapporte les propos d'un médecin spécialiste au sujet des transplantations cardiaques: "une bonne chose, certes, mais je ne crois pas que ce soit l'avenir ", contrairement aux organes mécaniques. Un autre médecin exprime son scepticisme vis-à-vis de la transplantation cardiaque et indique que l'«on déchantera dans une quinzaine d'années ${ }^{427}$ ». Omniprésent dans les années 1960, le problème persistant du rejet tempère l'enthousiasme suscité par les progrès chirurgicaux: "l'ennemi numéro un du chirurgien "greffeur" n'est pas la difficulté technique à vaincre mais bien le patient lui-même ${ }^{428}$ ». Les décès successifs des patients transplantés alimenteront le scepticisme d'une partie de la communauté médicale, comme en témoigne le compte rendu d'une conférence sur «les aspects médicaux et moraux de la greffe du cœur " qui décrit celle-ci comme une opération "qui reste une solution au caractère exceptionnel ${ }^{429}$ ».

Les interrogations médicales relatives aux transplantations de cœur humain et artificiel se prolongent dans les années 1970, comme l'indique un article établissant un bilan des transplantations cardiaques: "plusieurs équipes chirurgicales dans le monde en sont

4264 décembre 1967, 14.

42727 juillet 1968, 5 .

4286 septembre 1969, cahier 36 .

42916 octobre 1968, 18. La presse médicale montre également que les réserves ou interrogations émanent aussi de médecins non spécialistes de la transplantation (voir chapitre 4). 
même arrivées à douter de la valeur de ces opérations coûteuses pour la société, pénibles pour les malades et leurs familles ${ }^{\mathbf{4 3 0}}$ ». Mais dans le même article, le journaliste rapporte l'opinion optimiste d'un médecin qui estime que "bon nombre de difficultés s'apaiseront grâce à la recherche et que les greffes cardiaques seront, dans dix ans, aussi courantes que les autres interventions cardiaques pratiquées aujourd'hui ». Certains experts estiment ainsi que la difficulté majeure du rejet de l'organe sera surmontée à moyen terme. Prenant exemple sur les progrès réalisés dans la transplantation rénale, l'espoir est de rigueur pour les autres organes: «Une fois les méthodes au point, il n'est pas impensable d'envisager la création de banques d'organes comme il existe des banques de sang ${ }^{431}$. " D'autres discours sont beaucoup plus mesurés sur l'avenir des transplantations d'organes humains: "Déjà fort critiquées, les transplantations cardiaques se font beaucoup plus rares, dans l'attente, semble-t-il, des organes artificiels ${ }^{432}$. ${ }^{2}$ Les avis divergents quant au futur du cœur artificiel témoignent de cette période de stagnation scientifique qui rend incertain le développement de la transplantation. Les questionnements relatifs aux organes mécaniques seront encore présents dans les années 1980 dans un registre un peu différent de celui des années 1960 et 1970 durant lesquelles le développement éventuel des cœurs artificiels notamment est souvent imaginé dans l'optique de compenser le manque de greffons d'origine humaine.

\section{Politisation de la transplantation (1980-1998)}

La médiatisation accrue des transplantations d'organes à partir de 1980 tient en grande partie aux articles relevant de la catégorie "science et médecine» ( $\mathrm{n}=77 ; 45 \%$ des 170 articles de la période 1980-1998 - voir Graphique 3). Le thème des organes artificiels, notamment les implantations de cœur mécanique, y occupe désormais également une part substantielle $(\mathrm{n}=15)$. Les transplantations de William Schroeder et de Barney Clark font l'objet d'un traitement

43012 novembre 1971, 3.

43114 avril 1973, samedi littéraire, 225.

43223 novembre 1973, 3. 
médiatique similaire à la "chronique des opérés " qui concernait les premiers patients ayant reçu un cœur humain. Les années 1980 et 1990 relatent également toute une série de premières chirurgicales: greffe des deux mains, transplantations simultanées multiples (cœur-poumon, cœur-poumon-foie), xénotransplantations, transplantations de nouveau-nés, opérations inédites dans les hôpitaux suisses (transplantation de cœur, de foie, de poumon) pour ne citer que quelques exemples. La rhétorique de comptabilité et de record est également présente, bien que beaucoup moins prononcée qu'à la fin des années 1960. Les décennies 1980 et 1990 sont marquées par un regain d'activité de la médecine de transplantation qui s'explique principalement par les progrès considérables accomplis dans le contrôle des phénomènes de rejet de l'organe grâce notamment à la découverte par le laboratoire Sandoz à Bâle de la cyclosporine, un nouveau médicament immunosuppresseur.

Si du point de vue des articles médico-scientifiques, la période 1980-1998 ne diffère guère de celle de la fin des années 1960, elle se distingue par la fréquence notable des catégories "régulation institutionnelle» et "pénurie d'organes» (respectivement $\mathrm{n}=36$ et $25 ; 21 \%$ et $14,7 \%$ des 170 articles - voir Graphique 3). S'agissant de la «régulation institutionnelle », le $J d G$ rend principalement compte de l'actualité liée à la mise en place d'un cadre juridique, politique et sanitaire nécessaire au développement de la médecine de transplantation en Suisse (modalités du consentement, collaboration entre centres hospitaliers, création d'une coordination nationale de la transplantation ${ }^{433}$, ou encore élaboration d'une loi fédérale sur la transplantation d'organes ${ }^{434}$. L'importance quantitative de la catégorie « régulation institutionnelle» dans les années 1980 et 1990 est le témoin indiscutable d'une institutionnalisation et d'une politisation de toute une série de questions que pose la médecine de transplantation. Cette prise en charge du domaine des transplantations par les autorités sur le plan législatif, de l'organisation du système sanitaire

433 Voir chapitre 3.

434 La mise en place de cette régulation institutionnelle de la médecine de transplantation fera l'objet d'une forte médiatisation dans la presse romande entre 1998 et 2007 (voir Hammer 2012). 
et de la définition des compétences des différents acteurs concernés fait de la transplantation un sujet politique. Ce processus se double de la fabrication du don et de la transplantation d'organes comme problème de santé publique qui suppose non seulement l'implication des pouvoirs publics (Swisstransplant et l'OFSP en première ligne), mais aussi de la population. Cet aspect crucial, sur lequel nous revenons plus loin, se révèle parfaitement à travers la question de la pénurie d'organes, fait épidémiologique et argument rhétorique, par laquelle la transplantation devient un problème de société.

\section{Graphique 3}

\section{Répartition par année des catégories thématiques (1980-1998)}

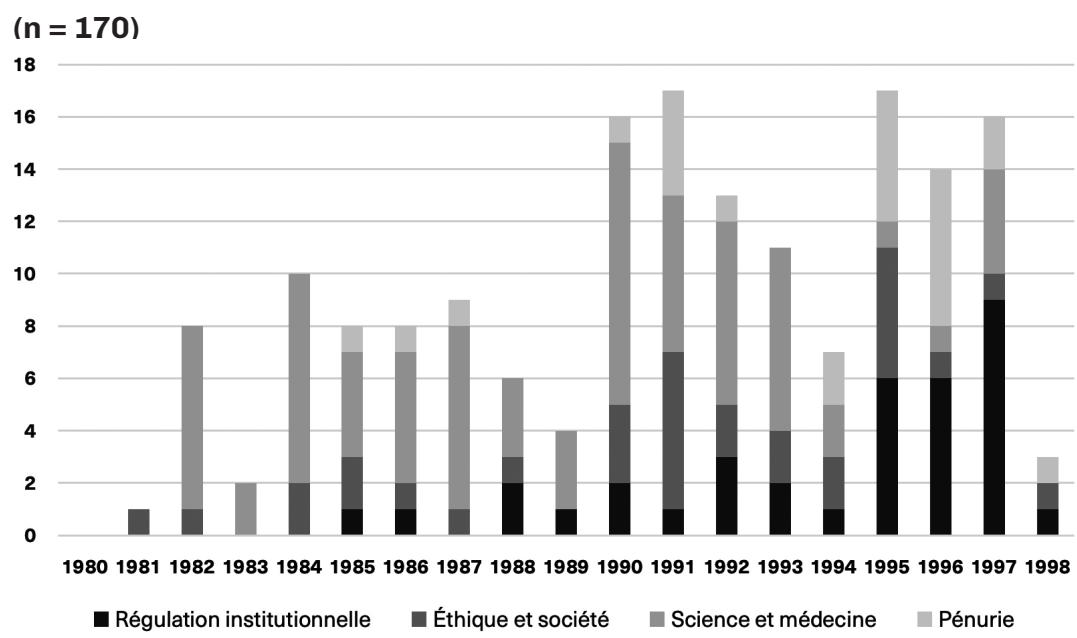

\subsection{Les enjeux éthiques}

Bien que le cadrage strictement médico-scientifique de la transplantation soit largement dominant dans l'ensemble du corpus, les questionnements sur la valeur sociale ou la légitimité de la transplantation ne sont pas absents. L'analyse a permis de dégager plusieurs enjeux éthiques significatifs dans le $J d G$ : la question de l'intégrité du corps et du statut de la personne, la justification de certaines transplantations, la mort cérébrale et le consentement lors 
de l'échange d'organes ${ }^{435}$. Si certains de ces enjeux sont associés à une période historique particulière, d'autres le sont beaucoup moins et tendent à être réactualisés au fil des innovations médicales.

\section{Intégrité du corps et de la personne:}

\section{un questionnement historiquement situé}

Les quelques articles qui abordent les questions philosophiques, morales ou existentielles des transplantations d'organes s'observent essentiellement à la fin des années 1960, à la faveur de l'écho retentissant qu'a connu la première transplantation cardiaque réalisée par Barnard. Plusieurs articles mentionnent les «problèmes moraux et humains ${ }^{436}$ » ou les "questions métaphysiques et éthiques ${ }^{437}$ ", sans nécessairement les développer, comme si, dans ce contexte, les "problèmes [que les greffes] posent [...] au point de vue éthique ${ }^{438}$ " étaient un fait commun.

Dans ce contexte, la question de l'impact de ces interventions médicales sur la notion de personne apparaît comme l'une des préoccupations morales identifiées. Pour autant, la question de l'intégrité de la personne et du statut des organes n'est discutée véritablement que dans un nombre restreint d'articles. On en trouve une première trace dans un "Feuilleton philosophique" qui rend compte en partie d'un ouvrage d'Aurèle David traitant de la frontière entre «personne» et «chose ${ }^{439}$ ». Le journaliste évoque la réification progressive des éléments du corps humain: «les techniques issues de la biologie et de la médecine conduisent de plus en plus à traiter le corps humain ou certaines de ses parties comme des choses"; "Que dire de ces "pièces détachées" que des greffes audacieuses transportent d'un individu sur l'autre et qui séjournent parfois dans

435 Les analyses de cette section portent sur l'ensemble des articles dans lesquels ces enjeux éthiques apparaissent, impliquant un sous-corpus plus large que la catégorie thématique "éthique et société".

43627 juillet 1968, 5 .

43729 juillet 1968, 3.

43824 septembre 1968, 14.

43914 février 1956, 4. 
des locaux intermédiaires en attendant preneur. » D'autres articles évoquent les bouleversements de l'utilisation des organes à des fins thérapeutiques sur la définition juridique de la personne; "la notion de la personne (corps humain) reçoit un coup sérieux de ces greffons vivants qui s'échangent comme des choses ${ }^{440}$ ».

Mais c'est en décembre 1967, à l'occasion de la première transplantation cardiaque, que l'on trouve l'expression la plus aboutie de cette interrogation fondamentale autour du statut de la personne humaine à travers une série de trois entretiens avec une personnalité différente. Inédite dans notre corpus par son format, cette série d'articles est en soi révélatrice de l'impact médiatique de l'opération de Barnard et des enjeux plus larges qu'elle pose. Si «cette opération présente, du point de vue technique, un intérêt exceptionnel, [qui] n'est pas douteux ", la justification de cette série tient dans le fait que "la greffe d'un cœur humain [...] soulève également, sur les plans religieux et moral, de délicats problèmes ${ }^{\mathbf{4 4 1}}$ ». L'intérêt de ces trois articles tient certes dans les réponses des interviewés mais aussi dans la nature des questions posées par le journaliste et dans l'identité des experts sollicités (un pasteur, un philosophe et un cardiologue). Les titres des articles - «Garant de la Création ou apprenti sorcier ${ }^{442}$ ? », «Préservez le structuralisme ${ }^{43}$ ! » et «A-t-on le droit? Question de probité $^{444}$ »- expriment sous une forme condensée les questions morales autour de l'intégrité de la personne transplantée qui sont abordées dans plusieurs autres articles parus principalement entre la fin des années 1960 et le début des années 1970.

«Dans quelle mesure les greffes organiques portent-elles atteinte à l'intégrité de l'être humain? À partir de quelle dose d'intervention l'individu, au sens moral du terme, court-il le risque de se voir profondément bouleversé? Quelle est la signification des réactions immunologiques de l'être humain? ", tels sont les "délicats problèmes »

4403 juillet 1958, 3 .

44114 décembre 1967, 13.

442 Idem.

44315 décembre 1967, 17.

44416 décembre 1967, 21. 
abordés dans ces trois articles. Pour le pasteur Jean-Marc Chappuis, «la technique des greffes [...] doit permettre à l'homme de s'épanouir plus heureusement [...], mais subsiste, encore intacte, la question de la continuité de la personne ${ }^{445} »$. Ces articles soulèvent la question du statut des éléments du corps humain (Les organes sont-ils des ressources thérapeutiques? Sont-ils des «choses »? À qui appartiennentils ?), mais aussi celle de la signification de l'échange d'organes entre êtres humains (Est-ce contre-nature? Jusqu'où chercher à vouloir combattre la finitude? Quel est le prix de la santé ?). La signification ontologique du rejet par l'organisme du nouvel organe constitue un autre problème soumis au pasteur: "Tout se passe comme si l'organisme préférait la mort plutôt que de devoir la vie à l'introduction d'un organe étranger. Quelle peut être la signification théologique de cette réaction physiologique fondamentale? » L'entretien prolonge la question de l'ordre divin et des lois de la nature en s'interrogeant sur le sens moral des transplantations en regard de la spécificité de l'homme d'un point de vue théologique. À cet égard, le pasteur estime que les transplantations, en tant que moyen de prolonger la vie, ne sont «pas forcément» conformes: "L'homme a, entre autres, l'obligation d'affronter lucidement la mort. Cela fait partie de son "vrai bien". "

Les réponses du pasteur et du philosophe montrent que si les "greffes humaines» interrogent, bousculent, voire perturbent les repères moraux habituels et les conceptions de la personne, il n'est pour autant guère à l'ordre du jour d'y renoncer. Pour le pasteur, la transplantation constitue une avancée irréversible mais dont le développement doit s'accompagner d'une perspective humaniste, à savoir "les assimiler au mieux de ce vrai bien de l'homme qui doit rester notre objectif majeur ${ }^{446} »$. Pour le philosophe, le renoncement éventuel aux transplantations n'a pas lieu d'être: "si à tort ou à raison on refusait du point de vue philosophique les greffes, il faudrait également s'interdire d'employer les électrochocs, l'insuline et la pharmacopée ${ }^{447}$ ». Si l'intégrité de l'être humain lui apparaît comme

44514 décembre 1967, 13.

446 Idem.

44715 décembre 1967, 17. 
étant un faux problème, les craintes du philosophe sont plus terre à terre, voyant dans le contexte de concurrence scientifique internationale un risque d'abus de la part des chirurgiens. Pour le philosophe, ce n'est pas la moralité de la transplantation en soi qui doit retenir l'attention, mais plutôt l'éthique médicale de ceux qui la pratiquent.

Le statut du corps humain constitue l'un des enjeux éthiques spécifiques des articles des années 1950 et 1960 puisqu'on n'en voit pratiquement plus trace par la suite, sauf lorsque le $J d G$ relate dans deux articles en 1990 le "débat sans précédent parmi les spécialistes américains ${ }^{448}$ " à propos du bébé médicament. L'un des articles cite l'avis d'un des experts qui dénonce la réduction du projet d'enfant à la seule finalité de son potentiel thérapeutique; "N'est-il pas barbare de faire naître un enfant dans le seul but de le mutiler, sous prétexte d'extraire de lui une substance-miracle ${ }^{449}$ ? » Dans le second article, en une, la journaliste offre au lecteur une position critique contre "l'instrumentalisation" du corps humain: "ce cas est limite dans la mesure où, si peu que ce soit, les parents (et les médecins) vont attenter à l'intégrité physique du bébé à naître [...] qu'on le veuille ou non, il s'agit d'une instrumentalisation, donc d'une certaine dégradation de la vie "; "De là à supposer que des bébés puissent un jour être engendrés puis avortés pour servir de greffons... Mais qui voudrait être guéri à ce prix ${ }^{450}$ ? 》 Bien que de manière moins explicite et plus politique, la critique de la réification du corps humain, et plus largement l'éthique de la transplantation, resurgira dans les années 1990 dans les articles dénonçant les cas de commerce et trafic d'organes.

\section{La justification des transplantations: enjeux médicaux et sociétaux}

La justification de telle ou telle opération concrète de transplantation constitue un autre enjeu significatif de la fin des années 1960. L'interview du cardiologue qui clôt la série d'articles évoquée ci-

44820 février 1990, 5.

449 Idem.

45010 mars 1990, 1. 
dessus aborde des aspects médicaux de l'intervention de Barnard, mais aussi des interrogations éthiques, à l'instar du problème "déontologique» suivant soulevé par le journaliste: "En général, un blessé qu'on amène, un soir dans un hôpital, et dont le cœur, par exemple, est "attendu" à quelques chambres de la sienne, par un autre malade, est-il assuré de se voir offrir toutes ses chances de survie, si ténues, si infimes soient-elles ${ }^{451}$ ? » Le cardiologue répond sur deux registres: la "probité » comme valeur cardinale du médecin d'une part, les compétences et techniques permettant de certifier la mort d'un individu d'autre part (voir plus loin). Le commentaire du journaliste montre bien que le ton n'est pas à une mise en cause des pratiques médicales mais plutôt à se faire le porte-parole des inquiétudes du public, tout à la fois légitimes et parfois non rationnelles: "Ces précisions contribueront sans doute à apaiser certaines craintes, injustifiées, certainement, mais compréhensibles, du grand public $^{452}$.» Le ton d'autres articles s'avère parfois moins conciliant à l'égard des médecins pratiquant des transplantations.

L'interrogation sur la légitimité de certaines transplantations transparaissait déjà en 1953 dans le compte rendu du drame de Marius Renard, un jeune homme victime d'un accident nécessitant l'ablation de son rein unique et qui reçut un rein de sa mère (don vivant). Dans un article sur "Les difficultés de la greffe", le biologiste Émile Guyénot évoque un léger doute sur le bien-fondé de l'opération: "tentative désespérée et peut-être légitime car le malade n'avait, par anomalie, qu'un seul rein qu'un accident venait de mettre hors service ${ }^{453}$.» La prudence est mise en avant en 1968 par les médecins allemands par rapport à leurs homologues américains: "les médecins allemands procèdent à un examen minutieux des chances de survie avant chaque greffe du rein et décident de tenter l'opération uniquement dans les cas où les conditions de succès semblent être réunies ${ }^{454}$.»

45116 décembre 1967, 21.

452 Idem.

45324 février 1953, 3.

4543 avril 1968, 18. 
La justification des premières transplantations cardiaques insiste également fréquemment sur la gravité extrême de l'état de santé du malade et sur la perspective d'une mort inéluctable à brève échéance: «Le professeur Barnard a indiqué un fait très important: outre son état cardiaque très grave, l'opéré était aussi diabétique. Et c'est en fait pour cette raison, parce qu'il n'avait plus que pour quelques semaines à vivre étant atteint par ces deux maladies à la fois que Washkansky a été choisi pour cette expérience unique dont l'issue reste incertaine ${ }^{455}$.» On retrouve cette rhétorique à propos de la première transplantation cardiaque réalisée en Suisse: «les chances de survie du patient étaient minimes. Malgré cela, le patient a tenu à subir l'opération, sachant qu'il n'avait plus que quelques semaines à vivre ${ }^{456}$." Un filet relatant la première transplantation cardiaque «en pays socialiste» livre la même justification: «Mme Horvathova était entrée à l'hôpital dans un état si critique que les risques d'une opération normale étaient aussi grands que ceux d'une greffe. C'est ce qui a décidé le professeur Siska à tenter l'opération ${ }^{457}$."

Le spectre d'opérations non justifiées médicalement apparaît de manière très nette dans le commentaire empathique du journaliste sur le refus du deuxième patient greffé par Barnard de tenter une nouvelle transplantation cardiaque; "facile d'imaginer l'appréhension du dentiste du Cap, de sa femme et de sa fille à la simple idée de rouvrir l'énorme cicatrice de Philip Blaiberg. Appréhension, et peutêtre aussi répugnance à servir - même si c'est pour sauver sa vie - de cobaye à une technique chirurgicale qui, malgré l'extraordinaire progrès qu'elle constitue, ne s'en est pas moins soldée par dix-huit décès sur vingt-cinq interventions. Lassitude sans doute enfin pour un homme qui avait pu interpréter son sursis comme une guérison et qui, après six mois, constate que la vie nouvelle qui semblait s'offrir à lui avec un cœur neuf se trouve à nouveau terriblement menacée ${ }^{458}$.» Ces propos témoignent d'un registre discursif qui fait davantage

4556 décembre 1967, 20.

45615 avril 1969, 3.

45710 juillet 1968, 12.

4588 juillet 1968, 12 . 
cas de considérations humaines que strictement médicales. Le journaliste présente en outre les progrès de la transplantation sous un angle ambivalent: une technique certes "extraordinaire» mais dont l'efficacité est mise en doute. L'interrogation sur l'attitude des médecins se lançant dans ces opérations n'est pas exceptionnelle. Un article consacré aux débats suscités par les transplantations cardiaques en Grande-Bretagne cite un homme politique suggérant que le corps médical devrait faire preuve de retenue: "les chances de succès d'une transplantation cardiaque sont si rares, qu'on ne saurait insister auprès des patients pour qu'ils se soumettent à une telle opération ${ }^{459}$.»

$\mathrm{Si}$, comme on l'a vu plus haut, le $J d G$ se fait l'écho de discours, à certains égards défensifs, visant à rassurer sur le caractère justifié des transplantations et sur le respect du patient, les chirurgiens peuvent aussi être décrits sur un mode laudatif qui met en avant l'audace ou le courage de celui qui franchit le pas dans un contexte où la transplantation fait débat. C'est le cas de la réaction d'un confrère du Pr Åke Senning, chirurgien cardiaque à Zurich et auteur de la première transplantation cardiaque en Suisse: «Il y a longtemps que le professeur Senning aurait pu tenter une telle intervention, mais vous savez à quel point les greffes du cœur sont discutées [...] il faut beaucoup de "culot" pour transplanter un cœur. Le chirurgien ne risque pas sa vie, mais bel et bien celle du patient ${ }^{460}$. " C'est d'une manière similaire qu'un journaliste résume la conférence publique d'un chirurgien et cardiologue en évoquant la "conscience professionnelle » et la «lucidité » des médecins «devant les problèmes moraux et psychologiques autant que techniques que pose l'opération de la greffe du cœur». Il est ainsi question du «grand courage des pionniers qui tentèrent les premiers cette expérience, comme celui de ceux qui acceptent de subir cette opération ${ }^{461}$ ».

Les interrogations sur la légitimité d'une transplantation ne réapparaissent, ponctuellement, que dans les années 1980 avec

4593 juin 1969, 16.

46015 avril 1969, 3.

46116 octobre $1968,18$. 
le cas particulier des implantations de cœur artificiel, et dans les années 1990 à propos des xénotransplantations.

Outre la question des coûts très élevés des cœurs artificiels, les problèmes «éthiques » qui sont mis en avant font écho à certains questionnements autour des premières transplantations cardiaques. En 1982, l'implantation d'un cœur artificiel dans la poitrine de Barney Clark fait l'objet de plusieurs articles. Dans l'un d'eux qui figure en une, la journaliste engage une réflexion critique sur «la question des rapports entre médecine et société » que soulève cette "première» médicale ${ }^{462}$. La polémique porte sur le sens de l'opération, dès lors que les "chances de survie sont faibles ", et qu'il s'agit d'une «survie artificielle» dont la qualité n'est guère enviable. La justification médicale et éthique de l'implantation d'un cœur artificiel est ainsi mise en doute. Mais c'est aussi la signification anthropologique d'une telle opération qui est questionnée, dès lors qu'elle illustre le culte de la santé et la technicisation des corps - « reflet des phantasmes prométhéens d'une civilisation qui rêve toujours de maîtriser la nature et de faire reculer aussi loin que possible les limites de la vie». Deux ans plus tard et encore en une, la même journaliste interroge l'utilité de l'opération réalisée sur William Schroeder "héros malgré lui de la chirurgie cardiaque de pointe ${ }^{463}$ ", compte tenu de l'incertitude forte du bénéfice thérapeutique du cœur artificiel et d'une qualité de vie très diminuée. Là encore, elle met en cause une décision médicale qui ne servait pas l'intérêt du patient: «Hyper-technique, hyper-coûteuse, aléatoire, cette médecine de l'exploit est peut-être inévitable. Qu'il nous soit permis de douter, et de son humanité, et de son utilité. » Un long article de mars 1988 réactive partiellement la question de la signification anthropologique de la transplantation de cœur artificiel: "Jusqu'où créera-t-on l'homme artificiel au lieu d'accepter la maladie et la mort ${ }^{464}$ ? »

46215 décembre 1982, 1.

46327 novembre 1984, 1.

46425 mars 1988, 2. 
Partie intégrante de la longue histoire médicale de la transplantation $^{465}$, le thème des transplantations d'organes d'origine animale sur l'homme apparaît de manière significative dans le corpus en 1984 avec l'implantation d'un cœur de babouin chez une fillette surnommée Baby Fae qui survécut trois semaines. Si cette opération donne lieu à une critique de la "médecine de l'exploit ${ }^{466}$ ", c'est dans les années 1990 que le thème des xénotransplantations apparaît à plusieurs reprises et sous un jour problématique. La transplantation de foie de babouin réalisée sur un homme en 1993 suscite des réserves: "Tous les spécialistes ne se montrent toutefois pas enthousiastes vis-à-vis de ce type d'intervention [...] [qui] s'interrogent sur le bien-fondé d'une greffe d'origine animale alors que la technique n'est aujourd'hui pas parfaitement maîtrisée et rappellent le manque d'informations sur ces opérations ${ }^{467}$. " Et si plusieurs articles associent étroitement la xénotransplantation et l'éthique dans les années 1990, c'est dans le contexte des discussions politiques autour de la future loi fédérale sur la transplantation. Les xénotransplantations sont évoquées comme un «sujet moralement et politiquement très délicat ${ }^{468}$ », qui suscite de "fortes craintes " et pose des "problèmes éthiques et sanitaires ${ }^{469}$ ». Pourtant, le $J d G$ relate également des discours d'acteurs médicaux présentant le développement des xénotransplantations comme l'une des mesures susceptibles à l'avenir de pallier la pénurie d'organes humains.

\section{La mort cérébrale:}

\section{incertitudes scientifiques et confiance sociale}

La question de la mort certaine de celui à qui l'on ôte un organe constitue un thème significatif de plusieurs articles de la fin des années 1960 et du début des années 1970. Pour bien situer ce contexte des incertitudes et interrogations autour de la mort

465 Schlich 2010.

46627 novembre 1984, 1.

46712 janvier 1993, 5.

4687 février 1997, 20.

4693 décembre 1997, 12. 
cérébrale, il convient de rappeler que la définition de ses critères diagnostiques est contemporaine des premières transplantations cardiaques, critères publiés pour la première fois en 1968 par une commission de la faculté de médecine de Harvard ${ }^{470}$.

L'évocation de la mort tient à deux questions, souvent liées: la capacité à définir scientifiquement la mort cérébrale, et la moralité du médecin impliqué dans le diagnostic de la mort. Interrogé sur la question de "la mort "cérébrale" ", Barnard invoque la validité scientifique de son travail et son intégrité professionnelle: "Si l'on enterre une personne un jour après le décès, il y a des parties de son corps qui sont encore vivantes. Pour moi, en toute conscience, je n'ai pas de problème. Je suis convaincu que tout ce que je fais est juste non seulement du point de vue scientifique, mais encore humain ${ }^{471}$. " Le diagnostic de la mort cérébrale fait ainsi l'objet de débats scientifiques et de doutes dont le $J d G$ se fait l'écho. Par exemple, dans un article consacré au $10^{\mathrm{e}}$ Congrès international d'angiologie organisé à Genève, la journaliste questionne: "A-t-on le droit [...] de déterminer arbitrairement - puisque les "cerveaux" de la greffe cardiaque semblent ne pas avoir arrêté une définition satisfaisante de l'instant de la mort: mort clinique ou mort du cerveau? - de déterminer, donc, le moment de l'ablation du cœur d'un être humain pour le greffer sur un autre être humain ${ }^{472}$ ?" L'emphase sur le fait que des «êtres humains» sont en jeu et sur l'incapacité des médecins à définir valablement la mort renvoie au lecteur une image écornée et peu fiable de la pratique de la transplantation. Les critiques de certains médecins à l'égard des critères de la mort cérébrale sont au centre d'un article évoquant les controverses que suscitent les transplantations cardiaques au Royaume-Uni: «En aucun cas la méthode des encéphalogrammes ne peut être considérée comme un moyen sûr pour distinguer un être mort d'un vivant ${ }^{473}$.»

470 "Report of the Ad Hoc Committee of the Harvard Medical School to examine the definition of brain death. A definition of irreversible coma", Journal of the American Medical Association, 205(6), 1968, 337-340.

4713 février 1968, 24.

47229 juillet 1968, 3.

4733 juin 1969, 16. 
La validité du diagnostic du décès du donneur tient une place significative dans le compte rendu de la première transplantation cardiaque en Suisse: "La mort cérébrale du donneur avait été établie avec certitude, a précisé le professeur Krähenbühl, avant d'indiquer les critères permettant de déterminer avec précision le moment de la mort: inconscience totale, disparition des réflexes, insensibilité des pupilles à la lumière, disparition des mouvements spontanés et disparition de l'activité cérébrale constatée à l'électroencéphalogramme ${ }^{474}$. " On retrouve la même insistance sur le constat du décès du donneur lors de la deuxième transplantation cardiaque en Suisse: "La mort du donneur - ainsi que le communique le service d'information des médecins suisses - était intervenue après que toutes les possibilités pour sauver sa vie soient restées vaines. Sa mort naturelle a été constatée par un médecin complètement indépendant du groupe de chirurgiens. Ainsi, les directives pour déterminer le diagnostic de la mort du cerveau, établies par l'ASSM, ont été rigoureusement suivies ${ }^{475}$. " Tant les précisions apportées par les médecins que le choix journalistique de reprendre les différents éléments techniques et de procédure traduisent certainement le souci de convaincre le public de la scientificité et du caractère éthique des conditions du prélèvement, et du même coup de prévenir les réactions négatives vis-à-vis d'une pratique médicale inédite. En effet, le parfum du scandale qui a entouré la médiatisation de la première transplantation cardiaque en Suisse alémanique - nous y revenons dans la section suivante - s'était accompagné d'un soupçon vis-à-vis du caractère désintéressé du diagnostic de la mort cérébrale ${ }^{476}$.

Dans les années qui suivent, la problématique de la mort cérébrale devient quasiment absente du corpus, à quelques rares

47415 avril 1969, 3. Dans l'ensemble du corpus, seuls deux autres articles donnent une définition substantielle de la mort cérébrale en 1991 et en 1995.

4759 juillet 1969, 12.

476 Bellanger, Steinbrecher et Obrecht 2002. Dans les articles des années 1960-début 1970 qui évoquent l'idée de mort cérébrale du donneur, l'expression "mort cérébrale" ou "mort encéphalique" figure rarement en tant que telle: "mort d'une hémorragie cérébrale", "arrêt de l'activité du cerveau du donneur", ou encore "mort d'une insuffisance encéphalique". Ce "flottement" lexical autour de la mort cérébrale est aussi et surtout de nature médicale (voir chapitre 4). 
exceptions près. Un long article daté du 4 mars 1972, consacré à la mort d'un point de vue philosophique, constitue une notable exception. Exposant les critères de la mort cérébrale, il indique que, malgré tout, «il ne peut y avoir de preuve absolue de la mort cérébrale, mais ces critères, admis par l'ASSM et par les pays qui nous entourent, forment la base d'une législation humainement possible en ce domaine ${ }^{477}$ ». On peut également citer un article abordant la question de la fiabilité des diagnostics de mort, partant d'un fait divers concernant des personnes qui seraient "enterrées vivantes" par erreur ${ }^{478}$. Enfin, on relèvera un article évoquant la perception de la mort cérébrale par le public ${ }^{479}$. On peut donc constater d'une part que la mort cérébrale est surtout médiatisée et discutée dans le contexte de la fin des années 1960, et d'autre part que ce thème ne réapparaît par la suite que de manière sporadique, par le biais d'articles donnant des informations scientifiques sur la définition et les modalités de la mort cérébrale.

\section{L'émergence du consentement comme question juridique et politique}

Dans le contexte des années 1960 caractérisé par une incertitude sur l'efficacité durable des transplantations cardiaques et par une sourde préoccupation autour du caractère inédit de telles opérations sur l'homme, la question du consentement n'est présente dans le $J d G$ que de manière très marginale. Cependant, parmi plusieurs des articles qui en font mention, le consentement apparaît comme un enjeu significatif du bien-fondé des transplantations ${ }^{480}$.

Ainsi, un article relatant la première transplantation cardiaque reprend l'un des points sur lesquels le chirurgien a mis l'accent: «le professeur Barnard a souligné que son équipe avait décidé [...] de ne prélever le cœur que sur un mort et seulement après que la

4774 mars 1972, samedi littéraire, 172.

47827 avril 1985, 16.

4792 novembre 1991, 27.

480 Sur la question du consentement dans le contexte des expérimentations menées au début du XXe siècle, voir Schlich 2010, 133-145. 
famille du défunt en eût donné l'autorisation ${ }^{481}$. » La précision du consentement lors du prélèvement figure également à propos de la deuxième transplantation cardiaque en Suisse: «Les parents du donneur ont été informés par avance et ont expressément donné leur consentement ${ }^{482}$.» On peut faire l'hypothèse que cette précision du consentement du donneur - absente du compte rendu de la première transplantation cardiaque en Suisse - n'est pas sans lien avec le scandale médiatique suscité par le Blick qui s'était fait l'écho de la colère de Paul Gautschi, ayant appris par les médias que le cœur de son fils avait été prélevé. Le quotidien à sensation zurichois s'était fendu d'un titre choc: «On a volé le cœur de mon garçon ${ }^{483}$ ! (Man hat meinem Bub das Herz gestohlen!).

Dans les années 1960, la préoccupation autour de la question du consentement ne concerne pas uniquement le donneur mais également, voire surtout, le receveur d'un organe. Dès lors que les transplantations, cardiaques notamment, constituent des thérapies encore largement expérimentales, c'est la question du risque lié à l'opération, et non le bénéfice éventuel, qui explique que le consentement du receveur soit spécifié dans plusieurs articles. Dans un long article de février 1963, le correspondant du $J d G$ à Londres relate la transplantation de rein d'un patient gravement atteint: «M. Lucas était dans un état vraiment désespéré quand, au début de décembre, les chirurgiens qui le soignaient lui proposèrent, en l'avertissant des risques qu'il courait, de lui greffer un rein naturel tiré du corps d'un homme d'âge moyen qui venait de mourir subitement d'une hémorragie cérébrale et dont la famille avait consenti au transfert. Il accepta ${ }^{484}$.» Dans le compte rendu de la première transplantation cardiaque en Suisse, il est question de "l'assentiment» du receveur et du fait que «les chances de survie du patient étaient minimes. Malgré cela, le patient a tenu à subir l'opération, sachant qu'il n'avait plus que quelques semaines à vivre », comme l'ont souligné les médecins lors de la conférence

4816 décembre 1967, 20.

4829 juillet 1969, 12.

483 Voir Bellanger et Steinbrecher 2002.

48420 février 1963, 1. 
de presse ${ }^{485}$. Dans ces exemples, le "consentement ${ }^{486}$ » renvoie à la problématique du risque, à l'assurance de ne pas être exposé à une opération expérimentale et à l'absence d'alternative thérapeutique. Les mentions du consentement dans les années 1960 tendent ainsi à être associées au fait que les transplantations sont des opérations de la dernière chance pour le patient, et dont le taux de succès est inconnu.

Jusqu'au début des années 1970, les rares mentions du consentement indiquent que cette question apparaît dans sa modalité la plus simple. Il n'est pas encore question de consentement présumé ou explicite, mais fondamentalement de s'assurer que le patient à qui est destiné l'organe et la personne prélevée ont exprimé leur accord. En outre, l'enjeu du consentement reste confiné à la relation médecinpatient. Dans le corpus, le consentement est en effet essentiellement évoqué en termes de protection des droits individuels, sans que la transplantation soit véritablement associée à un enjeu collectif, encore moins de santé publique. À cette époque, il n'y a aucune trace de l'idée que tout un chacun, de son vivant et en bonne santé, soit amené à prendre position sur l'éventualité d'être un donneur après sa mort. Ce n'est que dans les années 1980 que la notion de besoin collectif d'organes ou de manque de greffons émerge (voir plus loin).

À partir des années 1970, on observe un changement dans la mesure où la médiatisation du consentement est très largement déterminée par l'actualité institutionnelle. Cette question s'impose en effet progressivement comme un problème politico-juridique à la faveur de toute une série d'événements dont le $J d G$ se fait l'écho ${ }^{487}$. Ces événements marquent les premiers pas de la prise en compte de la transplantation dans les lois cantonales, notamment l'adoption des critères de la mort cérébrale ${ }^{488}$.

48515 avril 1969, 3.

486 La présence de termes comme "autorisation", "accord" ou encore "assentiment" montre que le concept de "consentement" ne s'est pas encore imposé.

487 Il s'agit, entre autres, du constat que le contexte juridique suisse est inadapté à la réalisation des transplantations cardiaques, et de la plainte en justice déposée par Paul Gautschi accusant le chirurgien d'avoir prélevé le cœur de son fils sans l'avoir consulté (article daté du 16 janvier 1970, 16).

488 Bellanger et Steinbrecher 2006, Tröhler 2004. 
L'état de la réflexion juridique dans les années 1970 suggère un déplacement sensible dans la manière d'appréhender la problématique du consentement au sens large, l'enjeu principal étant de trouver un équilibre entre les intérêts du receveur et du donneur. Le communiqué de presse consacré au congrès annuel de la Société suisse de droit pénal en 1970 met ainsi l'accent sur le compromis à trouver entre deux aspects conflictuels: «le respect dû à un mort et la possibilité de se servir de lui pour sauver une vie menacée. Les médecins et les juristes ont reconnu la nécessité d'établir de nouvelles lois tant pour protéger les particuliers que pour permettre à la science de sauver des vies humaines en appliquant des méthodes nouvelles ${ }^{489}$. Six ans plus tard, le compte rendu de la Journée juridique de la faculté de droit de Genève relate une préoccupation similaire: «De même que le défunt peut avoir, pour des motifs qui lui sont personnels, manifesté son refus de voir son corps utilisé pour une transplantation, les proches peuvent se sentir profondément atteints par l'usage qui est fait du cadavre »; "Le respect de ces sentiments doit être mis en balance avec l'intérêt du receveur à se voir pourvu d'un nouvel organe $\operatorname{sain}^{490}$.» Ce qui est nouveau ici, c'est l'affirmation que la transplantation constitue une thérapie, un bénéfice médical pour le receveur d'une part, et que l'intérêt de ce dernier est érigé à la hauteur de l'intérêt du donneur et de ses proches d'autre part. Cette recherche d'un compromis entre différentes valeurs est également mise en exergue dans un article daté du 21 septembre 1984: «Le prélèvement d'organes sur un cadavre soulève des questions éthiques importantes: comment concilier les intérêts de la science, des malades, et la protection de la liberté de chacun de disposer de son propre corps ${ }^{491}$ ? »

À partir des années 1970, le droit et le politique s'emparent de manière plus prononcée de la définition du consentement et des principes éthiques fondamentaux réglant la circulation des organes. À la fin des années 1980 et dans les années 1990, le $J d G$ se fait

48927 avril 1970, 7 .

49012 novembre 1976, 13.

49121 septembre 1984, 18. 
largement l'écho de débats politiques et juridiques sur la nature du consentement (présumé ou explicite) et sur le rôle de la famille par rapport à la position du défunt. Alors qu'ils étaient prégnants à propos des premières transplantations cardiaques à la fin des années 1960, les aspects psychologiques ou «moraux» du consentement du receveur ne sont plus guère évoqués ${ }^{492}$ dans un contexte où le contrôle médicamenteux du rejet a fait ses preuves. Comme le montre la section suivante, l'intérêt du receveur et la promotion du don occuperont à partir de la fin des années 1980 une place accrue dans les discours médiatiques avec la montée en puissance du cadrage du don d'organes en termes de pénurie.

\subsection{La montée en puissance du problème de la pénurie d'organes ou la publicisation du don}

Si le manque d'organes est un problème majeur de la médecine de transplantation, son évocation dans le $J d G$ s'avère relativement récente, au même titre que la signification qu'elle possède dans les discours contemporains des acteurs de la transplantation. Chronologiquement, la médiatisation du manque d'organes se caractérise par deux périodes clairement distinctes: les années 1945 à 1983 , où cette thématique est quasiment inexistante $(8$ articles recensés, dont l'un concerne la cornée), et la période 1984-1998 au cours de laquelle la notion de pénurie devient durablement un thème quantitativement important (56 articles l'évoquent) et acquiert progressivement la signification sociale et politique qu'elle revêt aujourd'hui ${ }^{493}$.

\section{Une donnée épidémiologique inéluctable}

Dans la première période, l'idée de pénurie renvoie essentiellement à un constat médical et épidémiologique. Par exemple, un

492 Cet aspect resurgit néanmoins ponctuellement avec la transplantation de cœur artificiel.

493 Les analyses de cette section portent sur l'ensemble des articles mentionnant le manque d'organes, et non uniquement sur ceux dont il s'agit du thème principal (i.e. la catégorie thématique "pénurie d'organes"). 
article daté du 20 février 1963 relatant les résultats prometteurs d'une transplantation de rein met en garde contre tout triomphalisme thérapeutique, notamment en raison du fait que «le nombre des "donneurs", vifs ou morts, appropriés est extrêmement faible ». Dans cet article, le manque de donneurs potentiels n'est qu'un facteur parmi de nombreux autres paramètres cliniques qui limitent le nombre de candidats dont le rein est susceptible d'être prélevé à bon escient pour un malade - cancer, maladie infectieuse, hypertension, compatibilité du groupe sanguin, alors que «le sexe du donneur est un facteur incertain encore». Dans cette période des débuts de la transplantation humaine, le manque d'organes apparaît sous les traits d'une pénurie programmée ${ }^{494}$. Là encore, le constat est neutre, et l'évocation de cette limitation future de la transplantation ne revêt aucune connotation critique ou publique, comme en témoignent des articles datés du 23 décembre 1967 (le «cœur humain, dont les disponibilités resteront forcément insuffisantes $\left.{ }^{495} »\right)$, et du 12 novembre 1971 («Pratiquement, tous ceux qui s’intéressent aux greffes d'organes estiment qu'il n'y aura jamais assez de cœurs disponibles pour sauver tous ceux qui en auront sans doute besoin $\left.{ }^{496} »\right)$. L'affirmation de cette pénurie programmée est l'occasion pour des médecins de réfléchir aux alternatives: d'une part ce que l'on appellera plus tard la xénotransplantation, d'autre part le développement d'organes mécaniques, à l'instar du directeur de l'Institut des greffes d'organes et de tissus à Moscou, engagé dans un programme américano-soviétique de réalisation d'un cœur artificiel: "Même si nous parvenons à résoudre tous les problèmes jusqu'ici sans solution concernant la transplantation des organes, il y en a un qui restera insoluble: le nombre des organes donnés pour les transplantations ne parviendra jamais à rejoindre le nombre des personnes qui en ont besoin ${ }^{497}$. »

494 L'analyse de la presse médicale montre que le thème du manque d'organes apparaît déjà dans les années 1950 comme une préoccupation chez certains transplanteurs (voir chapitre 4).

49523 décembre 1967, 17.

49612 novembre $1971,3$.

49715 février 1978, 9. Le lien entre la pénurie d'organes et la recherche d'alternatives comme les greffes d'organes d'animaux ne disparaît pas complètement par la suite, 
Si l'évocation du problème de la pénurie comme fait inéluctable en termes relativement neutres se raréfie dans les années 1980 et devient marginale dans les années 1990, c'est notamment parce qu'une autre signification émerge. Un article de 1978 préfigure tant l'importance quantitative qu'aura la question du manque d'organes humains dans le $J d G$ que la manière de cadrer le problème à partir de la fin des années 1980. Cet article introduit une rupture significative par rapport aux contenus des articles précédents par la mention des causes possibles du manque d'organes, ce qui implicitement pose le «déficit» (c'est le terme utilisé) de greffons comme un problème réversible. C'est donc la première fois qu'il est fait mention dans le corpus d'une explication du phénomène qui pointe deux facteurs: d'une part "les refus opposés par les familles ", qui dépendent "beaucoup de l'information sur les questions de transplantation", d'autre part «la difficulté d'obtenir des organes pouvant être greffés» qui vient "aussi des médecins travaillant dans des équipes de

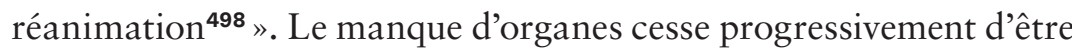
une donnée uniquement épidémiologique et médicale pour devenir aussi un fait social et politique. La problématique du nombre de greffons s'enrichit alors d'une nouvelle composante: le donneur potentiel non plus seulement dans sa dimension biologique (le corps dont on peut prélever des organes) mais en tant que citoyen appelé à se positionner sur le devenir de ses organes après sa mort. C'est à partir de la fin des années 1980 que se cristallisera ce nouveau cadrage de la pénurie d'organes.

\section{La pénurie d'organes: un problème de santé publique}

Un article de 1984 marque symboliquement le début de la deuxième période de notre corpus. De manière explicite, on trouve pour la première fois la qualification du manque d'organes en tant que "problème» qui revêt une résonance morale et de santé publique; «Le docteur [Jacques] Bernheim relève que les proches du défunt

à l'instar de cet article citant le Pr Philippe Morel: "Les xénotransplantations présentent des perspectives intéressantes, car nous n'aurons jamais assez de donneurs d'organes humains" [7 février 1998, 10].

4988 juin 1978, 12. 
endossent une lourde responsabilité en refusant que l'on utilise un organe à des fins thérapeutiques. En effet, c'est souvent la vie d'un être humain qui est en jeu [...]. M. Bernheim saisit l'occasion pour rappeler que le manque d'organes et de tissus pose actuellement un grave problème. Avis aux donateurs ${ }^{499}$... ${ }^{2}$ Le refus est problématisé non pas en regard de la psychosociologie de la famille du donneur mais en regard du pool des receveurs ainsi privés d'une chance de survivre. Et la mention du problème de la pénurie s'accompagne d'un appel au don. L'analyse des cinquante-six articles de la période 1984-1998 révèle un lexique diversifié mais cohérent dans la manière de décrire la situation comptable de l'activité de transplantation et dans la signification qui lui est attribuée.

Les caractéristiques de ce nouveau cadrage de la pénurie sont premièrement le recours très fréquent à l'objectivation statistique par différents indicateurs quantitatifs (nombre de donneurs par million d'habitants, nombre de transplantations effectuées, nombre de patients en attente de tel ou tel organe, nombre de patients décédés en liste d'attente, rang occupé par la Suisse au niveau européen). Un article de décembre 1985 chiffre la situation helvétique: "Il faudrait pratiquer chaque année 250 à 300 transplantations rénales en Suisse, a indiqué le professeur Felix Largiadèr, de l'Hôpital universitaire de Zurich. Toutefois, depuis des années, en raison du manque de donneurs, seules 150 transplantations peuvent être effectuées. Au centre de transplantation de Zurich, selon ce professeur, il y a au moins 130 patients qui attendent - certains depuis six ans - de pouvoir recevoir un rein ${ }^{500}$.» Autre exemple, un article de 1997 indique l'existence de près de 500 demandeurs d'organes: "Avec parfois une issue funeste: quarante-trois patients sont décédés l'an passé [...] avant d'avoir pu bénéficier d'une greffe salvatrice ${ }^{501}$. " Le sort du patient ayant besoin d'un organe change de perspective: son éventuel décès ou la longueur de l'attente est ainsi fréquemment associé, de manière symbolique, à la figure du non-donneur ou du

49921 septembre 1984, 18.

5005 décembre 1985, 13.

50112 avril 1997, 39 
donneur non déclaré davantage qu'à la maladie elle-même dont il est affecté ou l'absence de traitements alternatifs ${ }^{\mathbf{5 0 2}}$.

Deuxièmement, le problème du nombre insuffisant de greffons est très souvent assorti d'une qualification négative qui souligne la gravité de la situation: "on manque dramatiquement d'organes à transplanter ${ }^{503}$." Les chiffres en matière de dons sont "accablants ${ }^{504}$ ", et «le taux de refus des familles d'une personne décédée est anormalement élevé dans notre pays ${ }^{505}$ ». La pénurie d'organes constitue «un fait cruel ${ }^{506}$ ", une "situation catastrophique ${ }^{507}$ ». On observe aussi que les termes désignant le manque de greffons tendent à se fixer à la fin des années 1980. Si les termes "déficit» et «carence» apparaissent à quelques reprises dans notre corpus, les expressions "manque de donneurs", "manque d'organes» et «pénurie ${ }^{508}$ » sont les plus fréquentes et sont absentes avant 1984.

Le nombre insuffisant de greffons émerge donc comme un problème majeur auquel il convient d'apporter des solutions par des mesures spécifiques. C’est vers la fin des années 1980 que le manque d'organes apparaît dans le $J d G$ comme un problème public à part entière contre lequel il faut lutter, et qu'il n'est quasiment plus assimilé à un phénomène inéluctable, à une limitation «naturelle» de l'activité de transplantation. Par exemple, un article de 1991 décrit le «manque d'organes" comme un "problème» qui «limite [le] fonctionnement optimal» de la transplantation ${ }^{509}$. Corollaire de la thématisation du manque d'organes comme problème de santé publique, la nécessité d'améliorer le nombre de dons d'organes

502 Par exemple, "les donneurs se font en Suisse de plus en plus rares et les listes de personnes en attente d'une greffe s'allongent, quand ces derniers ne décèdent pas" [7 avril 1995, 22]. Voir aussi Stücklin 2008.

50315 octobre 1994, 28.

5042 novembre 1991, 27.

5055 décembre 1991, 28.

50625 novembre 1992, 23.

50710 septembre 1996, 14 .

508 Le terme "pénurie" apparaît pour la première fois dans notre corpus en 1986 et devient fréquent à partir de 1992.

5092 novembre $1991,27$. 
en Suisse devient un fait établi. Portés par les spécialistes de la transplantation mais aussi par la voix des journalistes, les discours volontaristes de lutte contre la pénurie sont alors très présents.

Cette thématisation s'avère étroitement déterminée par les événements institutionnels et communicationnels qui jalonnent la mise en place d'une organisation politique, juridique et hospitalière de la circulation des organes en Suisse. Plus particulièrement, les discours contenus dans le $J d G$ sur les causes du manque d'organes et les mesures pour y faire face sont largement à mettre sur le compte de l'importance croissante, à partir de la fin des années 1980, de Swisstransplant comme acteur dans l'espace public national ${ }^{510}$. Si les causes propres au fonctionnement hospitalier et aux pratiques médicales sont évoquées à quelques reprises dans le corpus, la pénurie d'organes est plus fréquemment associée à la problématique de l'information du public, comme en témoignent les propos souvent cités des responsables de Swisstransplant: "Le taux de refus des familles ou parents au moment du décès devrait pouvoir diminuer avec une meilleure information ${ }^{511}$ "; "l'augmentation des dons d'organe passe [...] par une information plus importante auprès du grand public ${ }^{512}$.» Évoquant les résultats d'une étude sur les facteurs de la pénurie, un article de novembre 1991 cite notamment les fausses croyances et représentations du public: "Clichés et fausses peurs ont poussé les gens à faire des amalgames complètement erronés ${ }^{513}$. "

Que les causes soient d'ordre cognitif, psychologique, émotionnel ou culturel, le manque de greffons apparaît principalement comme une question collective, voire comme un problème du public. Par exemple, à l'évocation du lancement de la carte de donneur de Swisstransplant en 1987, l'objectif est de «créer un réflexe de solidarité dans la population ${ }^{514}$ ». La promotion du don ou les appels au don sont souvent présents dans les articles: "Sensibiliser les

510 Voir chapitres 2 et 3.

51125 novembre 1992, 23.

51212 mars 1997, 1.

51325 novembre 1991, 21.

51415 octobre 1987, 19. 
personnes en bonne santé à devenir donneurs ${ }^{515}$ »; "Convaincre les personnes vivantes de donner leurs organes après leur décès ${ }^{516}$ "; «Par cette campagne Swisstransplant espère que le nombre de donneurs potentiels augmentera ${ }^{517}$.» Le titre d'un article daté de 1985 illustre mieux encore le lien entre la pénurie et la sollicitation du public comme levier privilégié pour la combattre: «Transplantation d'organes: on cherche des donneurs ${ }^{518}$ ! »

La montée en puissance de la pénurie d'organes dans les discours institutionnels et à travers les médias contribue ainsi significativement à donner une résonance publique et collective à la transplantation en créant un lien social entre celui qui a besoin d'un organe et la population en tant que collectif. "C'est une affaire de société. Nous sommes tous des donneurs ou des receveurs potentiels ${ }^{\mathbf{5 1 9}} »$ : l'article rapportant ces propos du Pr Philippe Morel, alors président du Comité exécutif de Swisstransplant, résume parfaitement ce mouvement de publicisation de la transplantation en tant que don d'organes.

\subsection{Conclusion}

À l'évidence, l'histoire de la transplantation se lit en partie à travers la presse. C'est le cas du développement médical, institutionnel et politique de la transplantation en Suisse et à Genève. Pour schématiser, les deux périodes dans lesquelles les transplantations d'organes font l'objet d'une médiatisation particulière correspondent effectivement d'une part à une actualité médicale inédite (les premières transplantations cardiaques à la fin des années 1960), d'autre part à la mise sur l'agenda politico-législatif des enjeux de la régulation sociétale du don d'organes (dès la fin des années 1980). Concernant les enjeux éthiques, qui occupent une place marginale dans le $J d G$, on a pu noter néanmoins qu'ils émergeaient à la fin

5157 février 1998, 10.

5167 février 1997, 20.

5179 décembre 1991, 20.

5185 décembre 1985, 13.

51910 septembre 1994, 12. 
des années 1960 dans le contexte des premières transplantations cardiaques pour devenir très peu visibles dans la décennie suivante, et pour réapparaître à partir des années 1980, à la faveur là encore d'opérations techniques inédites (implantation de cœurs artificiels notamment), mais aussi en raison de la politisation de la question de la transplantation en vue de l'élaboration d'une loi fédérale.

Ces enjeux éthiques autour des transplantations revêtent une forme différente entre le début et la fin de la période considérée. Schématiquement, dans les années 1960, ils apparaissent comme des questions «morales» ou philosophiques, voire théologiques. À partir des années 1980, il ne s'agit plus de questions «morales» mais bien "éthiques", ce qui reflète la montée en puissance de l'éthique dans le champ médical au niveau international. On peut observer aussi que certains questionnements de la fin des années 1960 ne se posent plus guère dans le $J d G$ dans la période récente: les organes sont-ils des choses? Qu'est-ce que la personne humaine? Qu'est-ce que le développement de la transplantation nous dit sur notre rapport civilisationnel à la mort, à la santé ?520 Si à la fin des années 1960 on pouvait encore s'interroger sur la légitimité de la transplantation d'organes, cela n'est plus le cas dès les années 1980 (cœur artificiel mis à part) qui se distinguent par des débats éthiques juridicisés et politisés. Il faut souligner que si le bien-fondé des transplantations est parfois questionné, notre corpus ne comporte aucune trace de contestation du principe de la transplantation humaine - ce sont plutôt les conditions et les modalités de la mise en œuvre de la transplantation qui sont parfois critiquées.

Si l'histoire des transplantations se lit donc en partie dans le $J d G$, il convient de rappeler avec force que le contenu et les discours de la presse quotidienne ne sont au mieux qu'un miroir partiel de l'actualité ${ }^{521}$. Si les médias constituent un acteur de l'espace public,

520 Comme nous l'avons évoqué dans l'Introduction, ces questionnements n'ont pas disparu des réflexions contemporaines sur le don d'organes, notamment dans les sciences humaines (voir par exemple Le Breton 2008, Moulin 1995, Boileau 2002, Sharp 2006, Gateau 2009, Veatch 2000). Pour une histoire de l'éthique de la transplantation, voir Jensen 2011.

521 Par exemple, la première transplantation rénale couronnée de succès réalisée en 1954 à Boston par le chirurgien Joseph Murray (entre frères jumeaux) n'est pas 
on peut s'interroger, dans notre cas, sur le rôle de la presse. Pour Christiane Kapitz, spécialiste en éthique de la communication, la logique médiatique de captation ${ }^{522}$ s'exerce au détriment d'une logique de mise en débat des aspects sociétaux des questions scientifiques: "Les greffes d'organes suscitent un intérêt très vif de la presse quotidienne, qu'elle traduit d'ailleurs davantage en termes de prouesse technique - ou d'échec - qu'en termes d'enjeux - humains, sociaux, économiques, politiques, juridiques ou éthiques ${ }^{523}$." Certes, on a pu constater l'importance du cadrage médico-scientifique du sujet des transplantations, mais si les enjeux éthiques ne sont pas absents, leur évocation par le $J d G$ tient le plus souvent à des événements extérieurs. On a toutefois pu relever des prises de position critiques dans quelques articles à l'égard d'opérations de transplantations particulières. Mais c'est surtout lorsqu'elle se fait le relais des discours des milieux de la transplantation et d'une "politique d'exhortation ${ }^{524}$ » que la presse devient un acteur décisif de l'espace public, a fortiori quand il s'agit de messages destinés à sensibiliser la population et à promouvoir le don d'organes. À cet égard, on peut relever que les discours de presse ont participé indéniablement au processus de publicisation et de mise en visibilité de la question de la transplantation, tout en soulignant l'impulsion décisive de l'émergence de Swisstransplant comme émetteur dominant et légitime des messages sur le don d'organes. Ce qui tendrait à soutenir l'hypothèse, par rapport au sujet des transplantations, d'une autonomie toute relative des discours de presse par rapport à l'environnement médical et politique. À cet égard, tout comme nous avons pu l'observer pour les années $2000^{525}$, on peut affirmer que la presse est davantage partie prenante de la construction d'un consensus social sur la valeur du don d'organes que de la mise en débat et la réflexion sur l'échange d'organes dans la société.

relatée par le JdG.

522 Charaudeau 1997.

523 Kapitz 2004, 177.

524 Steiner 2010.

525 Hammer 2012. 


\section{Conclusion de la Partie I}

\section{Régulation de la transplantation et émergence d'un problème public}

Vincent Barras et Alexia Cochand

Cette première partie a permis de mettre en évidence plusieurs éléments spécifiques de l'histoire de la transplantation dans le contexte suisse.

Tout d'abord, il apparaît clairement que la transplantation fait l'objet d'un développement non linéaire, façonné par les médecins des hôpitaux, sans l'appui d'une politique sanitaire qui aurait été établie préalablement entre les cantons ou par les autorités fédérales. Si les connaissances techniques constituent un prérequis fondamental au développement de la transplantation, la mise en place de cette pratique dans les hôpitaux nécessite une organisation spécifique, telle que l'aménagement de locaux, le signalement de donneurs potentiels ou encore la disponibilité de diverses catégories de soignant·e•s. On constate que, bien que la valeur thérapeutique de la transplantation ne soit jamais remise en cause, les critères économiques et logistiques propres à chaque établissement - surtout dans le cas des greffes cardiaques et hépatiques - jouent un rôle important. Sous l'impulsion des médecins, la mise en place des programmes est en effet l'objet de négociations, alors que la dynamique de développement dans les hôpitaux est essentiellement façonnée en termes budgétaires. À l'échelle nationale, c'est l'allocation d'organes 
entre les hôpitaux qui constitue l'enjeu organisationnel majeur par l'utilisation optimale des organes disponibles. L'élaboration de règles fixes et communes au sein de groupes de travail dès la fin des années 1960, ou la mise en place de la coordination nationale au début des années 1990 attestent d'un développement largement redevable aux médecins concernés en marge de réflexions politiques peu élaborées.

Les sources documentaires, et plus particulièrement les archives hospitalières peu exploitées à ce jour, permettent de dresser les grandes lignes de ce développement dans les hôpitaux suisses; elles posent toutefois certaines limites que l'histoire orale permettrait sans doute de dépasser. En effet, alors que le processus décisionnel au sein des hôpitaux échappe souvent à la mise par écrit, les entretiens rétrospectifs avec les protagonistes apporteraient des informations complémentaires tant sur le contexte de travail et l'ethos des chirurgiens transplanteurs dans cette période "pionnièreexpérimentale» que sur le fonctionnement hospitalier. Un certain nombre d'entretiens ont été menés dans le cadre de cette recherche; néanmoins, les informations recueillies ont été moins mobilisées que celles des sources écrites en raison d'un usage plus problématique, lié aux difficultés méthodologiques propres à l'histoire orale. Quels médecins choisir et selon quels critères établir ce choix ? À quel moment de la recherche mener les entretiens pour acquérir le plus d'informations exploitables? S'agit-il d'obtenir des réponses factuelles et précises pour pallier le manque d'informations? Faut-il au contraire privilégier l'évocation de souvenirs généraux en laissant la personne interviewée aborder pêle-mêle ce qui lui paraît rétrospectivement significatif et trier ultérieurement les informations recueillies? Comment ajuster l'intérêt épistémique du chercheur et les motivations personnelles de l'individu interviewé pour rendre les propos recueillis exploitables? Si ces questions nécessiteraient une réflexion approfondie dépassant largement les limites de cet ouvrage, elles laissent ouvertes de riches et pertinentes perspectives de recherches à approfondir.

Cette première partie permet également de mettre en lumière l'image publique de la transplantation et sa diffusion tant dans la 
communauté médicale non spécialisée que dans la sphère publique. En suivant, grâce à l'analyse des revues médicales généralistes et de la presse écrite, le passage de la thématique de la transplantation dans l'espace médiatique par l'analyse des revues médicales généralistes et de la presse écrite, c'est sa représentation dès son origine que l'on peut ainsi saisir.

Bien que les revues médicales et le Journal de Genève constituent des corpus médiatiques très différents, notamment lorsque l'on considère leur fonction et leur public, certains événements constituent des points de convergence dans la médiatisation. Les deux types de médias laissent en effet apparaître une forte préoccupation concernant les conditions du prélèvement et la survie des receveurs. Aux prises avec un dilemme constitué par le primum non nocere et l'incitation à tenter quelque chose pour un malade, le chirurgien pose, avec ses tentatives souvent infructueuses, les jalons d'une éthique fondée sur des valeurs fortement personnalisées, comme le courage et l'audace. Dans un contexte de grande incertitude médicale, les médecins transplanteurs contribuent eux-mêmes à renforcer la représentation de la transplantation comme se déroulant hors du cadre d'un système défini.

Bien que la question délicate de l'équilibre à trouver entre les intérêts du donneur et les besoins du malade émerge dans les deux corpus, c'est la presse générale qui contient les plus nombreux exemples de considérations morales sur le principe même de la technique des greffes et de sa signification sociétale, philosophique ou existentielle, et en particulier des interrogations sur l'intégrité du corps, la notion de personne et le statut des parties du corps humain. Chronologiquement, ces considérations se concentrent sur les périodes proches d'événements marquants, comme les premières transplantations rénales dans les années 1950, et surtout la première transplantation cardiaque en 1969. Lorsque certaines revues médicales générales abordent également ces événements, les questionnements éthiques au sens large sont peu présents, laissant davantage place au fait médical et aux données techniques.

Enfin, cette partie permet de suivre l'évolution de la transplantation comme enjeu politique et comme problème public en Suisse. 
Dans le domaine politique, la prise en charge par l'ASSM de la définition des critères de mort cérébrale puis des problèmes éthiques et légaux liés à la réalisation des transplantations ainsi que les jugements rendus par le Tribunal fédéral dans les années 1970 contribuent à laisser une grande liberté aux cantons dans l'organisation des transplantations. Confiée tacitement aux médecins des hôpitaux habilités, l'organisation prend progressivement forme au début des années 1990 dans le sillage de la création de Swisstransplant. Alors que l'implication de la sphère politique reste marginale jusqu'à la fin des années 1990, la fondation prend en main la gestion de la distribution des organes tout en jouant un rôle essentiel dans la diffusion d'une image rassurante de la transplantation tant pour le monde politique que pour le grand public.

À partir de 1999, alors que l'article constitutionnel sur la médecine de transplantation confère aux autorités fédérales le mandat de réguler et d'organiser l'activité de la transplantation, les prérogatives établies jusque-là par les acteurs du terrain, tels que les groupes de travail, Swisstransplant et les hôpitaux, sont bousculées. Les tensions et frictions engendrées à cette occasion poussent la fondation à élaborer de nouvelles stratégies, comme la réorganisation interne et le changement de conception dans la répartition des organes. Par cette forme de compromis qui la conduit à renoncer à l'omniprésence de médecins dans les structures exécutives, la fondation s'efforce de conserver son statut d'acteur clé dans la régulation et l'organisation de la transplantation et d'obtenir une reconnaissance politique en évitant une trop grande intervention de l'État.

L'émergence du don d'organes en tant que registre privilégié pour considérer le volet public de la transplantation et se constituer en problème de santé publique est réalisée avec la création de Swisstransplant et l'institutionnalisation progressive de la transplantation. Par la question de la pénurie d'organes, la transplantation est envisagée en effet comme un problème de société sous la forme du don d'organes. Si la préoccupation à l'égard de la pénurie est déjà présente dans les années 1950 et 1960, elle est appréhendée comme un problème inhérent à la transplantation elle-même. Avec la création de Swisstransplant, le don d'organes devient un problème 
public qui fait l'objet d'une promotion au moyen de diverses initiatives, telles que les messages et supports d'informations, et d'une présence médiatique accrue. Si ces moyens visent à l'information du public et à la promotion du don, c'est la pénurie qui devient elle-même au début des années 1990 un argument des discours publics. Progressivement, elle se transforme en un fait documenté et commenté, visible dans l'espace social, et un argument central d'interpellation et de sollicitation des bons sentiments de tout un chacun.

Les analyses de cette première partie montrent ainsi que le développement des techniques de transplantation s'est inscrit dans une régulation complexe et progressive, qui tient au caractère innovant de ces techniques, aux exigences de coordination d'un système efficace d'échanges des organes, mais aussi aux particularités du contexte institutionnel helvétique. À cet égard, il est remarquable que l'acteur clé de l'histoire institutionnelle de la transplantation soit un organisme paraétatique. La fondation Swisstransplant s'est en effet affirmée au sein de dispositifs tant fédéraux que cantonaux qui se sont déployés dans un deuxième temps, et dont les liens de collaboration avec les hôpitaux et l'État se seront renforcés et adaptés en fonction de l'organisation nationale et internationale du don d'organes et de sa reconnaissance croissante comme enjeu de santé publique. 



\section{Partie II. \\ Le don d'organes \\ comme enjeu \\ de mobilisation \\ associative}





\section{Chapitre 6}

\section{L'espace associatif du don d'organes}

\section{Raphaël Hammer et François Kaech}

Cette seconde partie de l'ouvrage présente les résultats du volet sociologique de la recherche, consacré à l'analyse du rôle des associations de transplantés par rapport au don d'organes comme enjeu de santé, de société et comme problème public. L'objectif n'était pas de produire une série de monographies associatives mais bien de cerner l'ensemble des différentes associations concernées, sans évacuer pour autant leurs singularités. Ce choix d'un regard englobant résulte de la multitude d'associations existant en lien avec la question du don d'organes et de la fragmentation de l'engagement collectif. S'il y a certes une Association suisse des transplantés (AST) qui regroupe le plus grand nombre de membres, elle n'a pas le monopole de la représentation politique et légale des personnes directement touchées par la greffe. Pendant une certaine période, plusieurs petites associations ont eu une visibilité médiatique locale parfois forte et ont régulièrement participé à des actions publiques, faisant ainsi preuve d'un «activisme » plus important que d'autres groupes de patients transplantés. S'intéresser à l'espace associatif du don d'organes témoigne aussi de la volonté de comprendre la mobilisation collective, en partant de l'hypothèse qu'elle est traversée par des traits communs, en termes de logique d'engagement et de fonctionnement, de domaines d'actions, de stratégies ou de conceptions 
du problème. Analyser l'espace associatif du don d'organes plutôt qu'un ou quelques groupements en particulier implique aussi que les relations entre associations peuvent être marquées par des rapports de pouvoir et de concurrence, des conflits, tout autant que par des liens de coopération.

Ce chapitre introductif expose les caractéristiques générales de l'espace associatif du don d'organes en Suisse romande, et présente l'étude de terrain que nous avons menée. Après avoir précisé la délimitation de l'espace associatif, nous retracerons rapidement l'émergence historique des associations de transplantés afin de situer le contexte contemporain de leur rôle et de leurs activités. Nous décrirons ensuite les différentes associations et fondations actives dans les années 2010, ainsi que leurs caractéristiques formelles et de fonctionnement. Enfin, nous rendrons compte des principaux aspects méthodologiques de la recherche.

\subsection{Délimitation de l'espace associatif du don d'organes}

L'espace associatif du don d'organes a été défini comme l'ensemble des organisations légalement constituées (sous forme d'association ou de fondation ${ }^{526}$ ) qui mènent des actions ou proposent des activités en lien avec la transplantation d'organes cadavériques exclusivement. Ce premier critère de délimitation tient au fait que les enjeux sociopolitiques et de santé publique sont particulièrement prégnants dans le don d'organes post mortem, c'est-à-dire dans le cas d'une personne en état de mort cérébrale ${ }^{527}$. Cette modalité du don d'organes est en effet dotée d'une charge émotionnelle très

526 Les articles 60 à 79 et 80 à 89 du Code civil suisse définissent les bases légales d'une association et d'une fondation. Dotée de statuts écrits, une association est obligatoirement constituée d'un comité et d'une assemblée générale réunissant tous les membres une fois par année. Le comité comporte en principe un président, un secrétaire et un trésorier. Une association est neutre sur les plans politique et confessionnel, et sans but lucratif. Constituée par un acte authentique, une fondation se compose d'un conseil de fondation composé de trois personnes (physiques ou morales) au moins et d'un conseil de révision. Elle implique un capital initial de 50000 francs. Les deux types d'entité se fixent un but à atteindre et sont en principe composés de bénévoles.

527 La question du don d'organes à cœur arrêté (DCD) constitue une problématique tout à fait spécifique qui n'est pas considérée ici. 
marquée puisque l'éventualité d'un prélèvement intervient dans une situation dramatique du décès généralement inattendu et brutal d'un proche. Rappelons également que la compréhension cognitive et phénoménologique de la notion de mort cérébrale pour les familles et pour la population générale est souvent difficile ${ }^{528}$, et que la transplantation d'organes venant d'une personne décédée engage des questions symboliques et anthropologiques fondamentales ${ }^{\mathbf{5 2 9}}$. Ces enjeux font l'objet de débats récurrents aux niveaux politique, légal, social et éthique. Enfin, d'un point de vue médical, rappelons qu'à l'exception du rein et d'une partie du foie, la transplantation d'organes n'intervient que sur un donneur décédé. Les autres modalités d'échange de parties du corps humain en tant que ressources thérapeutiques ne sont donc pas prises en considération, excluant les associations en lien avec le don d'organes entre personnes vivantes, le don de tissus (la cornée par exemple) ou de cellules souches du sang. Ces autres formes de dons constituent des enjeux moins politisés et débattus, moins médiatisés et font l'objet d'une mobilisation associative moins importante ${ }^{530}$.

Un deuxième critère de délimitation a été géographique: nous avons ciblé les associations localisées et actives en Suisse romande, en incluant certaines associations nationales dotées d'une antenne en Suisse romande et/ou dont les activités couvrent la partie francophone du pays. La prégnance de la question du don d'organes (en tant qu'expérience personnelle ou en tant que cause à promouvoir) dans les activités et buts de l'association nous a conduits à écarter celles qui sont centrées sur des pathologies spécifiques pour lesquelles la transplantation est souvent le seul traitement ou la meilleure option thérapeutique ${ }^{531}$. Enfin, nous avons exclu les organisations profes-

528 Boileau 2002, Ralph et al. 2014.

529 Le Breton 2008, Sharp 2006, Bateman 2002.

530 Pour le don vivant, il existe une Association suisse des donneurs vivants d'organe (ASDVO), créée en 2004 à Zürich. On notera également l'existence de Keradonum, une association en lien avec la greffe de cornée basée en Suisse alémanique. Enfin, il faut mentionner l'Association Suisse romande des greffés de la moelle osseuse et son homologue en Suisse alémanique.

531 À titre d'illustration, nous n'avons pas retenu la section romande de l'Association pour l'information et la recherche sur les maladies rénales d'origine génétique (AIRG), 
sionnelles dont les activités s'adressent avant tout au monde médical, à l'instar du Comité national du don d'organes (CNDO) dont le rôle principal est de promouvoir cette pratique au sein de l'hôpital, ou de la Fondation lausannoise de transplantation d'organes (FLTO) qui vise à soutenir la recherche médicale, la formation professionnelle et la qualité des soins. Dans cette même logique, nous n'avons pas retenu des organisations tournées essentiellement vers le soutien à la recherche médicale, à l'exemple de la Fondation Respirer dans le domaine de la mucoviscidose et de la transplantation pulmonaire.

\subsection{Quelques repères historiques}

Selon les sources consultées détaillées plus loin, la création des premières associations remonte au milieu des années 1970 avec la Société suisse des patients souffrant d'insuffisance rénale (SSPIR) et l'Association genevoise des insuffisants rénaux (AGIR) en 1975, puis l'Association des dialysés du canton de Vaud quelques années après, qui deviendra l'Association vaudoise des insuffisants rénaux (AVDIR). Leurs homologues dans les cantons du Valais (Association valaisanne des insuffisants rénaux) et du Jura (Association jurassienne des malades insuffisants rénaux - AJMIR) apparaissent quelques années plus tard, respectivement en 1982 et 1988. Si les premières associations sont liées au rein, c'est le reflet de l'histoire de la médecine de transplantation qui connaît ses premiers succès cliniques dans les années 1960 avec la greffe rénale. À l'origine, les associations pionnières ne sont pas des groupements de patients transplantés mais de malades atteints d'une pathologie rénale plus ou moins sévère. Si pour ces malades la transplantation d'un rein constitue déjà une éventualité probable et un traitement efficace, le don d'organes comme cause sociale n'est pas (encore) au centre des actions de ces associations mais constitue un objectif parmi d'autres. Au début du mouvement associatif dans les années 1970, l'une des préoccupations majeures consiste à faciliter l'accès des malades à la dialyse et à leur fournir une aide financière. Ainsi,

qui présente surtout une vocation d'entraide et d'information (la promotion du don d'organes dans l'espace public ne figure pas comme objectif). 
lors de sa création, l'Association valaisanne des insuffisants rénaux milite avant tout pour la création de nouveaux espaces de dialyse, notamment dans le Bas-Valais, et entend apporter un soutien financier aux malades habitant loin du centre hospitalier de Sion, qui sont contraints de faire de longs et coûteux déplacements pour accéder aux lieux de dialyse. L'aide financière proposée par les premières associations visait aussi à soutenir les patients transplantés qui devaient assumer de leur poche des coûts importants liés à l'opération que les assurances ne prenaient alors pas en charge.

Les années 1980 et 1990 constituent une seconde période significative du développement de l'espace associatif, caractérisé par l'apparition d'associations autres que liées au rein et qui tendent à mettre davantage l'accent sur la promotion du don d'organes auprès de la population. Ainsi, l'Association suisse des transplantés du cœur et de poumon, plus connue sous le nom des As de Cœur, est fondée en 1986 en Valais. Ses buts étaient multiples: «encourager la solidarité et la convivialité, familiariser le public au don d'organes, favoriser l'exercice physique et fournir des conseils pour la réinsertion professionnelle. » Dans un article de presse, son président fondateur soulignait que «le but primordial [...] est d'assurer une aide aux futurs transplantés en sensibilisant l'opinion publique au don d'organes: nous devons rechercher des donneurs ${ }^{532}$ ». Cet extrait montre, nous y reviendrons, que la constitution de la population comme "public» dans le discours associatif apparaît au même moment que dans les discours médicaux, tels que la presse les relaie comme nous l'avons signalé au chapitre 5. À la fin des années 1980, l'aide financière aux patients demeure une préoccupation importante dans la mesure où certains traitements ne sont pas couverts par l'assurance maladie. Dans la décennie suivante, de nouveaux groupements font leur apparition: l'Association sportive suisse des transplantés ${ }^{533}$, l'association des transplantés du foie Trans-Hepar

53224 Heures, 6 juin 1987, 7.

533 L'Association sportive suisse des transplantés mettait l'accent sur la pratique du sport dans le cadre de manifestations nationales et internationales ("pour le plaisir" et "retrouver une bonne condition physique"), et sur la promotion publique du don d'organes. 
(ou Amicale suisse des greffés du foie) fondée en 1992 à Berne, et l'association Phoenix dont le but était de promouvoir la cause $\mathrm{du}$ don d'organes et de regrouper différents collectifs partageant le même objectif. Dans ce contexte de multiplication du nombre d'associations, des intentions d'alliance s'expriment déjà. Certaines se concrétisent à l'instar de la création, en 1990, du Groupement des insuffisants rénaux romand (GIRR), rassemblant les associations des cantons romands, et dont l'ambition est de «soutenir et [...] aider les insuffisants rénaux, [...] informer sur l'insuffisance rénale et ses conséquences et surtout [...] promouvoir le don d'organes ${ }^{534}$ ». Ce n'est que quelques années plus tard que sera fondée l'Association neuchâteloise des insuffisants rénaux (ANIR) en 1997.

Durant les années 2000, troisième période significative, on assiste à une forte dynamique associative avec la création de plusieurs nouvelles entités: la fondation ProTransplant en 2001; PromOrgane, la fondation Passez-le-Relais et la fondation Tackers en 2002; l'Association fribourgeoise des insuffisants rénaux (AFIR) en 2004; l'association À Cœur Ouvert en 2005, et enfin la Fondation AGIR en 2007. On peut également mentionner la création de la fondation Fairtransplant en 2005 par des médecins transplanteurs dans le canton de Genève, à la suite de la première Journée mondiale en faveur du don et de la transplantation d'organes, fondation qui a œuvré plusieurs années pour la promotion du don d'organes dans l'espace public. Cette décennie est marquée par deux fusions significatives qui aboutiront à la plus grande association suisse de personnes transplantées: Les As de Cœur et Trans-Hepar se regroupent pour donner naissance en 2006 à l'Association suisse des transplantés (AST), en poursuivant leurs champs d'activité initiaux. Cette fusion était motivée par les difficultés financières et le manque de bénévoles, et par la volonté d'améliorer la qualité des prestations auprès des membres. La seconde fusion intervient en 2010 lorsque l'Association sportive suisse des transplantés est absorbée par l'AST pour en devenir une composante à part entière.

534 Le Confédéré, 14 septembre 1990, 2. 
Au cours des quelque quarante ans d'action associative en lien avec le don d'organes en Suisse romande, on observe de manière très schématique que les premières associations dans les années 1970 sont principalement tournées vers les besoins, notamment financiers, des patients dialysés, puis vers les besoins des patients transplantés d'autres organes. À partir de la fin des années 1980, la promotion du don d'organes dans la population prend une importance accrue, en tant que finalité principale ou objectif complémentaire aux missions d'aide. C'est donc progressivement que les associations et fondations s'emparent de la question du don d'organes comme problème social et s'attachent à agir auprès du public. La période des années 2000, qui fait l'objet de l'analyse des chapitres qui suivent, se caractérise ainsi par un développement significatif de l'espace associatif dans lequel l'enjeu public du don d'organes devient une préoccupation notable.

\subsection{La situation contemporaine}

Au moment de notre enquête (2012-2015), l'espace associatif romand est constitué de dix associations et de quatre fondations actives. Nous décrivons ci-après leurs principales caractéristiques factuelles et leurs buts généraux ${ }^{535}$ dans ce contexte temporel, en présentant d'abord les associations en lien avec la dialyse et la transplantation rénale, puis les associations couvrant tous les organes concernés par la transplantation, et enfin les fondations à proprement parler.

\section{Les associations en lien avec le rein}

Une caractéristique de l'espace associatif réside dans l'existence d'une association liée à l'insuffisance rénale dans chaque canton

535 Afin d'alléger le texte, nous n'avons pas indiqué systématiquement les multiples sources d'information utilisées dans cette section: entretiens avec les acteurs associatifs, statuts, plaquettes de présentation, bulletins d'information, sites internet ou encore articles de presse. Les données relatives au nombre de membres et à la composition des comités correspondent au moment de l'enquête. 
(Genève, Fribourg, Vaud, Valais, Neuchâtel et Jura) et d'une association faîtière ${ }^{536}$.

Association neuchâteloise des dialysés et transplantés (ANeDiT)

Présidée par un transplanté rénal à la retraite et fondée en 2013 à Marin, l'ANeDiT fait suite à l'Association neuchâteloise des insuffisants rénaux (ANIR) qui avait failli disparaître en raison de conflits internes et de démissions en son sein. Ce renouveau tient à l'initiative de l'un des membres du comité qui a «soumis aux anciens membres [de ANIR] l'idée de repartir dans une nouvelle voie et sur de nouvelles bases ». La nouvelle appellation traduit la revendication généraliste de l'association en s'ouvrant aux transplantés de tous organes, bien que la majorité de ses 70 membres soient des transplantés rénaux. Les buts de l'ANeDiT sont «de regrouper, d'informer, de soutenir et de défendre les intérêts des insuffisants rénaux (dialysés ou pas) et des transplantés de toutes sortes d'organes", et de "sensibiliser le monde politique et médical ainsi que la population neuchâteloise à l'importance du don d'organes ». L'aide psychosociale constitue également un objectif affiché, notamment en lien avec les «interrogations » et "difficultés » liées à la transplantation: "c'est aussi un lieu où les futurs greffés peuvent échanger et s'informer auprès de ceux qui ont déjà vécu la transplantation, dans le but de dédramatiser la période de l'attente et des ambivalences face à cette problématique ". Le comité est composé de six personnes, dont une infirmière coordinatrice de transplantation. L'association dispose d'un site internet et d'une page Facebook.

\section{Association jurassienne des malades insuffisants rénaux (AJMIR)}

Créée en 1988 à Delémont à l'initiative d'un petit groupe de malades, l'AJMIR vise à regrouper les personnes affectées d'une maladie rénale. Son but consiste à fournir une aide matérielle aux personnes tant dialysées que transplantées, notamment par l'organi-

536 Plus de la moitié des patients transplantés en Suisse chaque année sont des transplantés rénaux. Environ $75 \%$ des patients inscrits sur liste d'attente d'une transplantation sont des malades en attente d'un rein. 
sation d'un service de transport des patients à l'hôpital du Jura pour les séances de dialyse (sites de Porrentruy et Delémont). Lors d'un entretien, l'un des membres du comité décrit ainsi les raisons qui ont animé la création de l'association: «essayer d'aider dans le quotidien de chacun, chacune, pour aider, pour accepter cette maladie [...], pour essayer de rassurer les familles, pour leur dire que c'est une maladie chronique bien sûr, où il faut aller trois fois par semaine en dialyse, mais que finalement il y a un aboutissement qui est la transplantation. "Si la vocation de l'AJMIR réside principalement dans le soutien moral et logistique aux insuffisants rénaux du canton du Jura, elle vise aussi à participer à des actions de sensibilisation au don d'organes. L'AJMIR compte une soixantaine de membres. Le président, un transplanté rénal, est en fonction depuis 2007 et est membre de l'association depuis 1999. Une page internet présente brièvement l'association et la composition du comité.

\section{Association valaisanne des insuffisants rénaux et transplantés} (AVIRT)

Créée en 1982 sous le nom de l'Association valaisanne des insuffisants rénaux (AVIR), elle change son orientation en 2006 en intégrant les patients transplantés et devient l'Association valaisanne des insuffisants rénaux et transplantés (AVIRT). Selon ses statuts, l'AVIRT a pour but "l'amélioration de la situation des malades d'insuffisance rénale ou d'autre(s) organe(s) en Valais, spécialement l'encouragement et l'assistance morale des malades par des contacts et une aide réciproque". L'association mentionne notamment les objectifs suivants: «l'encouragement des possibilités de tout traitement médical de l'insuffisance d'organe(s) chronique en s'informant sur les développements techniques et les recherches; la création de possibilités et l'encouragement de toute action sur le plan des droits de la médecine, de l'organisation et de l'éthique, visant à mettre la transplantation à la portée de toute personne remplissant les conditions nécessaires; l'encouragement du don d'organes; une information sur les différents états de l'insuffisance rénale et d'autre(s) organe(s). » Dès sa création, l'association a participé à des actions de 
promotion du don et de sensibilisation du public aux problèmes liés à l'insuffisance rénale. Présidée par un transplanté rénal, elle compte quelque soixante-dix membres ordinaires et édite une brochure d'information interne. L'association ne dispose pas de site internet.

\section{Association fribourgeoise des insuffisants rénaux (AFIR)}

Fondée en 2004 et présidée par une patiente transplantée, l'AFIR est composée d'un comité de quatre personnes et compte une dizaine de membres au moment de l'enquête, contre une cinquantaine auparavant. Les buts de l'association sont de regrouper des personnes atteintes de pathologies du rein, promouvoir l'accès aux traitements, défendre les intérêts des patients concernés, améliorer l'information du public sur la médecine de la transplantation, et sensibiliser le public à la problématique du don d'organes. Comme plusieurs de ses homologues, l'AFIR souhaite officiellement élargir sa base en s'ouvrant aux patients transplantés des autres organes. Elle ne dispose ni de site internet ni de lettre d'information.

\section{Association vaudoise des insuffisants rénaux (AVDIR)}

Fondée en 1977, l'AVDIR représente les intérêts des personnes dialysées ou des patients transplantés rénaux, ainsi que leurs proches, dans le canton de Vaud. L'association est également ouverte aux personnes intéressées par l'insuffisance rénale et aux membres du personnel soignant. Ses objectifs consistent principalement à «aider et promouvoir la recherche, la dialyse et la transplantation». L'association a aussi une mission d'information des membres aux niveaux médical et diététique, et offre une aide matérielle concrète en louant un appartement de montagne situé à proximité d'un centre de dialyse. En plus de ces missions initiales, l'AVDIR a cherché à développer des relations de partenariat avec les soignants (néphrologues, membres du personnel infirmier, pharmaciens) afin de renforcer l'information aux patients. Au moment de l'enquête, l'association, présidée par un transplanté rénal, compte un peu plus de soixante-dix membres et ne dispose pas de site internet. 


\section{Association genevoise des insuffisants rénaux (AGIR)}

Fondée en 1975 et présidée par un patient retraité greffé du rein, l'AGIR rassemble des patients en situation d'insuffisance rénale et des patients transplantés du rein dans le canton de Genève. Les buts de l'association sont les suivants: "renforcer les contacts et la solidarité entre les personnes dialysées et greffées; apporter une aide morale et un soutien social à ses membres et à leurs proches; permettre l'échange d'information sur le vécu de l'insuffisance rénale; soutenir financièrement les membres qui en ont besoin; mener des actions d'information sur la dialyse et de sensibilisation au don d'organes et à la transplantation. » L'AGIR organise également des séances régulières d'exercice de musculation et d'équilibre pour les membres, au nombre d'une soixantaine. Elle dispose d'un site internet hébergé sur le blog d'un médecin néphrologue genevois.

\section{Société suisse des patients souffrant d'insuffisance rénale (SSPIR)}

Créée en 1975 et présidée par une patiente transplantée, la SSPIR (en allemand: VNPS pour Verband Nierenpatienten Schweiz) a pour vocation de représenter "des personnes de tout âge qui souffrent d'une insuffisance rénale, qui doivent être traitées par dialyse ou qui ont subi une transplantation rénale ". Rassemblant environ 1500 membres, la SSPIR est une association faîtière qui regroupe plus d'une dizaine d'associations cantonales d'insuffisants rénaux des trois régions linguistiques. Les membres peuvent faire partie de la SSPIR à titre individuel ou via l'une des associations régionales. L'association «met tout en œuvre pour préserver au mieux la qualité de vie des patients insuffisants rénaux et de leurs proches et pour représenter et défendre leurs intérêts ». Le comité comprend un médecin. Dotée d'un site internet bilingue, elle publie un journal environ deux fois par an depuis 2012, rédigé en allemand, en français (et en italien depuis peu), dont le but est notamment d'informer "sur les avancées médicales et pharmaceutiques qui concernent l'insuffisance rénale et ses traitements ». La SSPIR propose aussi de répondre à des questions des patients en collaboration avec des 
spécialistes des maladies et de la transplantation rénales. La SSPIR inclut deux sous-groupes de patients: l'association des parents d'enfants souffrant de maladies rénales et le groupe de travail « reins kystiques».

\section{Les associations "généralistes"}

Association suisse des transplantés (AST)

L'AST est créée en 2005 par la fusion de deux associations (As de Cœur et Trans-Hepar) dont elle reprend les champs d'activité (le président des As de Cœur devient président de l'AST). Elle regroupe environ quatre cents membres, majoritairement des personnes transplantées et quelques proches. Le comité inclut des représentants des trois régions linguistiques. Les objectifs de l'AST sont les suivants: "faire connaître le succès de la transplantation et ainsi promouvoir le don d'organes; soutenir les patients en liste d'attente; renseigner toutes les personnes qui souhaitent obtenir des informations au sujet du don d'organes et de la transplantation; aider éventuellement certains transplantés à retrouver une vie normale en les accompagnant pour régler certains problèmes administratifs et financiers ». Grâce à l'intégration de l'Association sportive suisse des transplantés (ASST) en 2010 qui en devient le secteur Sport, l'AST organise et participe à de nombreuses activités sportives, notamment de compétition au plan international (Jeux européens pour transplantés, Jeux mondiaux d'été et d'hiver pour transplantés). Le groupe sportif de l'AST «a pour but d'encourager en particulier les personnes transplantées à pratiquer des activités sportives ». L'AST dispose d'un site internet et produit deux fois par an un bulletin d'information interne en allemand et en français, et en italien depuis quelques années.

\section{À Cœur Ouvert}

Créée en 2005 à l'initiative de deux transplantés du cœur, À Cœur Ouvert œuvre dans les cantons du Valais et de Vaud tant pour l'information et la promotion du don d'organes que pour le 
soutien psychologique des personnes en attente de transplantation et des patients transplantés. Si l'association compte une dizaine de membres et un comité de plusieurs personnes, ce sont les deux fondateurs qui en ont été les principaux animateurs durant une dizaine d'années, autant par leurs diverses activités sur le terrain que par leur visibilité dans les médias. L'association ne possède ni journal interne ni site internet.

\section{PromOrgane - Association suisse pour la promotion du don d'organes}

Fondée en 2002 à Genève par un transplanté rénal et son épouse journaliste, PromOrgane vise à lutter contre la pénurie d'organes. Le but principal et initial de l'association consistait en «l'appui de la promotion pour le don d'organes afin d'augmenter le nombre de donneurs », par le biais d'actions artistiques, culturelles et sportives. La poursuite de ces objectifs était animée par le souci de coopérer activement avec divers acteurs du don d'organes (Swisstransplant en particulier et d'autres associations). L'information de la population constitue l'axe prioritaire de l'association: "Il est nécessaire d'améliorer les connaissances du grand public comme il s'avère tout aussi indispensable de mieux sensibiliser les jeunes aux possibilités multiples de la greffe d'organes "; "Il faut rendre cette information plus simple, plus naturelle et continuer à inciter les citoyens à porter sur eux la fameuse petite carte facilitant le don de leurs organes ». PromOrgane a été particulièrement active entre 2002 et 2011 dans la région genevoise, et ses actions ont souvent été couvertes par la presse locale. Selon l'évaluation d'un membre du comité, communiquée lors d'un entretien, PromOrgane rassemble environ cent cinquante membres. Le comité est composé d'une dizaine de personnes, dont Philippe Morel (alors médecin-chef du service de chirurgie viscérale aux hôpitaux universitaires de Genève - HUG). Après plusieurs années à la tête de PromOrgane, la fondatrice cède la présidence au vice-président, assistant social au service de transplantation des HUG. L'association a un site internet et publiait un bulletin interne plusieurs fois par année. 


\section{Les fondations}

\section{ProTransplant}

Créée en 2001 à l'initiative d'un médecin, ProTransplant est une fondation genevoise qui, par le biais d'organisation de conférences, soirées de gala et de campagnes publiques, "soutient les actions liées à la promotion, à la recherche et au développement du don et de la transplantation d'organes». ProTransplant prévoit également une aide matérielle, administrative et sociale aux familles de donneurs ou receveurs en situation précaire, ainsi qu'un soutien psychologique aux transplantés ou donneurs. ProTransplant est liée à Swisstransplant en tant qu'antenne locale et aux HUG - la responsable du comité d'action et cheville ouvrière des activités de la fondation y occupe la fonction de coordinatrice de transplantation. Le Conseil de fondation est présidé par un avocat et inclut plusieurs médecins des HUG. La fondation dispose d'un site internet.

\section{AGIR - Fondation pour l'aide aux malades chroniques à mobilité} réduite et la promotion du don et de la transplantation d'organes

La Fondation AGIR (Fondation pour l'aide aux malades chroniques à mobilité réduite et la promotion du don et de la transplantation d'organes) - homonyme de l'association AGIR - a été créée en 2007 à Genève à l'initiative notamment d'un patient transplanté du rein et du pancréas, conseiller en santé et action sociale au service de transplantation des HUG. Il avait été président de l'association AGIR dans les années 2000 et avait plusieurs années auparavant fondé les associations ASGREP (Association suisse des greffés rein et pancréas) et Phoenix. Les buts de la Fondation AGIR sont principalement l'aide aux malades chroniques à mobilité réduite par un service de transport à bas prix, et la "promotion du don et de la transplantation d'organes » par le biais de diverses actions de sensibilisation de la population, en lien notamment avec des événements sportifs. La fondation est née dans le cadre de la mise sur pied de la Transplant Cup - un événement à caractère sportif de promotion du don d'organes impliquant plusieurs associations d'insuffisants 
rénaux. Dans ce contexte, la création de la fondation visait d'une part à surmonter les écueils liés à la coopération interassociative, et d'autre part à associer des représentants du monde médical dans l'organisation de l'événement. Le Conseil de fondation est composé de plusieurs médecins des HUG. La fondation dispose d'un site internet.

\section{Passez-le-Relais}

La fondation Passez-le-Relais a été créée en 2002 à Montreux à l'initiative d'une mère dont le fils, victime d'un accident mortel de la route, fut donneur d'organes. Les buts principaux de la fondation sont les suivants: "distribuer en Suisse une carte de donneur d'organes dont les données seront enregistrées dans un registre informatisé; intensifier la promotion du don d'organes; organiser des campagnes d'information, des actions de parrainage et des journées de rencontre avec le public ", par le recours notamment à des personnalités jouant le rôle d'"ambassadeurs ", comme indiqué sur une brochure de présentation. L'originalité de cette fondation tient dans son premier objectif: recenser tous les donneurs potentiels dans une base de données informatique consultable par les hôpitaux universitaires dans l'optique de réduire la pénurie d'organes. La fondatrice a renoncé à son emploi pour se consacrer entièrement à la cause du don d'organes à travers Passez-le-Relais. Le Conseil de fondation comporte plusieurs personnalités publiques, émanant notamment du monde médical et politique.

\section{Tackers}

Créée en 2002 et basée en Valais, la fondation Tackers (Transplant Adventure Camps for Kids) est un projet de Swisstransplant qui est porté par une patiente transplantée du foie. Elle organise régulièrement des activités sportives, en particulier des camps de ski en Suisse à l'attention d'enfants transplantés et de leurs proches venant du monde entier. L'objectif principal consiste à «donner la possibilité aux enfants transplantés du globe de participer à l'aventure d'une vie et à une expérience dont ils se rappelleront pour toujours ", 
et à "créer une amitié et des liens pour que ces enfants et leurs familles puissent s'entraider et partager leurs expériences ». Ce type d'expérience a aussi pour mission de développer l'autonomie des jeunes patients transplantés et de favoriser leur épanouissement en démontrant "aux enfants qu'ils peuvent faire les mêmes choses que les autres enfants "normaux" peuvent accomplir». Ces camps, à travers leur couverture médiatique notamment, visent aussi à montrer au public les effets positifs d'une transplantation. La fondation dispose d'un site internet et bénéficie du soutien de personnalités internationales.

Pour compléter la description du paysage associatif dans le domaine du don d'organes, précisons que de nombreux groupements de patients sont également actifs dans les deux autres régions linguistiques. Concernant celles qui sont basées en Suisse alémanique ou qui y exercent l'essentiel de leurs activités, une demi-douzaine d'associations cantonales en lien avec l'insuffisance et la transplantation rénale sont recensées et membres de la SSPIR. Sans être exhaustif, on peut mentionner également des groupements comme Novaria (Association suisse des greffés pulmonaires), Kids Kidney Care (Association d'entraide pour les enfants atteints de maladie rénale et leurs proches), Verein Eltern Nierenkranker Kinder (VENK) (Association des parents d'enfants souffrant de maladies rénales), Elternvereinigung für das Herzkranke Kind (EVHK) (Association des parents pour les enfants atteints de maladies du cœur), Elternvereinigung lebererkrankter Kinder (EVLK) (Association des parents d'enfants malades du foie), Verein für Organspende Schweiz (Association suisse pour le don d'organes). En outre, une association basée dans le Valais (Organja), créée en 2013 et composée essentiellement de jeunes, est engagée dans la sensibilisation publique au don d'organes. Enfin, quatre associations sont actives dans le canton du Tessin: l'Associazione ticinese per pazienti di insufficienza renale (ATPIR), le Club Amici Swisstransplant, Vivere il trapianto - une association d'entraide -, et Insieme per ricevere e donare orientée vers la diffusion d'information sur le don d'organes auprès des jeunes principalement. 


\section{Caractéristiques générales de l'espace associatif suisse romand}

Il convient de remarquer tout d'abord que l'espace associatif du don d'organes inclut, au moment de l'enquête, des groupements présents dans l'ensemble des cantons romands (Genève, Vaud, Valais, Neuchâtel, Fribourg et Jura). Soulignons ensuite l'hétérogénéité à plus d'un titre de cet espace associatif. Du point de vue de l'ancienneté premièrement puisqu'il est composé autant de collectifs pionniers, nés dans les années 1970 et 1980, que de collectifs récents, créés dans les années 2000. Deuxièmement, la taille des associations varie considérablement: si certaines ne comptent que quelques membres, d'autres font état de plusieurs centaines d'adhérents. Cela n'est pas sans incidence sur l'ampleur de la zone d'influence. Excepté l'AST et la SSPIR qui visent explicitement une représentation nationale, ainsi que la fondation Tackers qui a en partie une orientation internationale (les camps d'hiver sont destinés à des enfants de tous pays), le rayon d'action revendiqué est essentiellement régional ou cantonal (les statuts de plusieurs associations en lien avec le rein mentionnent explicitement le canton comme ancrage géographique de référence). Le nombre restreint de membres affecte aussi le mode de fonctionnement dès lors que dans leur grande majorité les associations reposent sur l'engagement de malades, de quelques proches et de bénévoles. Comme noté précédemment, historiquement la création de la plupart des associations et des fondations résulte d'initiatives profanes, en l'occurrence de patients transplantés ou en attente de transplantation. De fait, les associations du don d'organes sont peu ou pas du tout professionnalisées. Le bénévolat constitue une caractéristique majeure du phénomène associatif en général: «le bénévolat, comme don de son temps et de son énergie sans attente de rétribution matérielle, est une valeur centrale de la vie associative $^{537}$.» S'agissant de la composition des groupements, à quelques exceptions près, le nombre de personnes actives dans les comités s'avère plutôt modeste, voire minime. Les professionnels de la santé (médecins ou membres du personnel infirmier) sont globalement 
peu présents dans les comités des associations alors qu'ils sont souvent bien représentés dans les conseils des fondations, compte tenu de leur notoriété, expertise et influence sociale ou politique. La plupart des associations correspondent à un modèle d'organisation largement répandu dans le domaine de la santé, à savoir des "petites associations de bénévoles, fonctionnant grâce à l'énergie énorme déployée par un noyau de quelques personnes très fortement impliquées, soutenues souvent d'assez loin par des membres plus ou moins nombreux mais peu actifs ${ }^{538}$ ".

Élément central du fonctionnement d'un collectif, l'aspect financier constitue un autre facteur de différenciation des associations. Les ressources, généralement limitées, proviennent essentiellement des cotisations des membres et de dons privés, et dans de rares cas des revenus personnels des membres fondateurs. De manière accessoire et ponctuelle, quelques associations enregistrent des entrées financières grâce au revenu d'activités propres (vente de produits, repas de soutien) ou d'actions caritatives, mais surtout grâce au soutien de la Loterie Romande, de firmes pharmaceutiques, d'hôpitaux, d'entreprises locales, de commerçants, d'associations professionnelles ou d'autres groupements. Les associations ne reçoivent généralement pas de subventions publiques, et lorsqu'elles existent, elles sont occasionnelles et d'un montant modeste. Swisstransplant ne finance pas directement les associations de patients mais fournit du matériel logistique pour la tenue de stands notamment (brochures, ballons, banderoles...). Si quelques associations ne rencontrent pas de problèmes de trésorerie, plusieurs font état de moyens faibles et d'une recherche continue de fonds pour développer ou maintenir leurs activités. Parmi les associations incluses dans notre étude, deux ont été confrontées à de sérieuses difficultés en raison de dettes impayées ou de fraudes qui ont occasionné des poursuites judiciaires et des condamnations. L'influence des caractéristiques des associations et de leur fonctionnement sur leur capacité de mobilisation sera abordée dans le chapitre 9. 
Enfin, il faut souligner que dans leur grande majorité les associations et fondations poursuivent les mêmes buts généraux: d'une part la défense des intérêts des membres, qu'ils soient transplantés ou en attente de transplantation (les proches peuvent aussi être concernés), sous la forme d'aides diverses; d'autre part la promotion du don d'organes dans la société ou la sensibilisation de la population à la thématique. Chaque groupement tend à décliner ces objectifs de manière spécifique en mettant l'accent sur l'un ou l'autre de ces deux domaines d'action. Alors que la recherche médicale peut être activement soutenue, sur le plan financier notamment, par des associations de patients dans le cas du sida, de la myopathie ou d'autres pathologies ${ }^{539}$, cet objectif est très marginal au sein de l'espace associatif du don d'organes et s'avère principalement assumé par des fondations spécialisées et professionnelles. Sans exclure d'autres facteurs explicatifs, cette situation indique que le problème du don d'organes n'est pas médical mais sociétal aux yeux des collectifs de patients, mais aussi des milieux de la transplantation et des pouvoirs publics.

\subsection{Données, méthodes d'enquête et objectifs}

Afin de décrire l'espace associatif en Suisse romande et analyser la dynamique d'engagement par rapport au don d'organes comme problème public, nous avons établi un dispositif d'enquête fondé sur plusieurs sources d'information.

Le corpus principal de données empiriques est constitué d'entretiens semi-directifs menés avec 31 personnes membres des 14 associations et fondations décrites ci-dessus. Toutes les personnes interviewées ont donné leur consentement explicite. La répartition de ces acteurs associatifs par groupement est la suivante: ANeDiT (1), AJMIR (2), AVIRT (1), AFIR (2), AVDIR (3), AGIR (2), SSPIR (1), AST (9, dont 5 appartenant à la section sport), À Cœur Ouvert (2), PromOrgane (2), fondation ProTransplant (1), fondation AGIR (1), fondation Passez-le-Relais (3) et fondation Tackers (1). Pour 
chacun de ces groupements, au moins une personne assumant des responsabilités dirigeantes a été interviewée. Au total, la majorité des personnes enquêtées ont des responsabilités de direction ou de gestion en tant que président (12), vice-président (4), secrétaire (2), membre du comité (1) ou responsable d'un comité d'action (1). Les 11 autres ont le statut de simple membre.

À notre connaissance, l'enquête couvre de façon exhaustive les associations et fondations actives en Suisse romande dans le domaine du don d'organes. Notre corpus inclut ainsi la grande majorité des acteurs associatifs engagés publiquement et médiatiquement au cours des dernières années, et permet une analyse de l'ensemble de l'espace associatif romand du don d'organes des années 2000. Si nombre des interviewés peuvent être considérés comme des figures importantes de la mobilisation profane pour le don d'organes, c'est aussi parce que certains ont fondé une ou plusieurs associations antérieures à celle dont ils font partie au moment de l'enquête. Précisons que les adhésions multiples ne sont pas rares dans notre corpus, à l'instar d'une interviewée membre d'une association locale et active dans le comité de l'association faîtière ${ }^{540}$. Enfin, plusieurs interviewés ont été membres à divers titres d'autres groupements liés au don d'organes dans un passé plus ou moins récent, permettant ainsi de multiplier et croiser les données. Sur le plan sociodémographique, les 21 hommes et 10 femmes rencontrés ont une moyenne d'âge de près de 59 ans - le plus jeune a 28 ans et le plus âgé 78 ans. Près d'un tiers des interviewés sont retraités, plusieurs sont au bénéfice complet ou partiel de l'assurance invalidité, et la grande majorité appartient à la classe moyenne.

Réalisés par François Kaech, les entretiens abordaient, outre les questions sociodémographiques usuelles, les thèmes suivants: l'implication et la carrière associatives, les caractéristiques et buts de l'association, la perception du problème du don d'organes en Suisse, les activités concrètement réalisées, ainsi que les relations entre associations et aux autres acteurs de la transplantation. À l'exception de quelques entretiens de près de trois heures, leur durée

540 Cette porosité au sein de l'espace associatif n'exclut pas l'existence de rapports conflictuels et de tensions (voir chapitre 9). 
moyenne était de une heure trente à deux heures, signe à la fois de la disponibilité des personnes sollicitées, de la richesse des échanges et d'un climat de confiance entre l'intervieweur et l'interviewé. Tous les entretiens ont été enregistrés, puis transcrits verbatim et anonymisés, et enfin codés à l'aide du logiciel Atlas.ti. L'exploitation des données a suivi la méthode des "catégories conceptualisantes » d'inspiration constructiviste qui combine des procédures déductives et inductives, permettant ainsi la prise en compte de catégories issues des questions de recherche initiales et de catégories émergeant des données ${ }^{541}$. L'analyse des entretiens visait à restituer de manière compréhensive et contextualisée l'expérience et la signification de l'engagement des interviewés dans l'action collective en lien avec le don d'organes, mais aussi à documenter les associations sur un plan descriptif.

Compte tenu du microcosme que constitue l'espace associatif romand du don d'organes et de la petite taille de plusieurs associations, la confidentialité des personnes rencontrées a été assurée en limitant au strict nécessaire les informations relatives à leur profil. Outre l'attribution d'un pseudonyme, nous avons pris le parti de citer les interviewés en mentionnant d'une part leur statut dans l'association ( «comité» ou «membre ${ }^{542}$ »), d'autre part la catégorie du groupement d'appartenance ("association en lien avec le rein", abrégé " $\mathrm{AR}$ » dans la suite du texte, "association généraliste » [AG] ou «fondation» [FD]). Lorsque cela est pertinent pour l'analyse, des caractérisations plus précises sur le groupement seront fournies. Dans le cas où nous citons des propos tenus publiquement (dans la presse notamment) ou des extraits de textes publiés (plaquette de présentation ou journal associatif par exemple), nous indiquons les noms du locuteur et du collectif. Pour éviter la répétition des termes «association » et «fondation", nous utiliserons dans la suite

541 Paillé et Mucchielli 2003.

542 Sous le terme "comité", nous englobons les personnes faisant partie de l'organe exécutif (ou "responsables associatifs"), à savoir celles qui ont le statut de président, viceprésident, membre du comité, secrétaire ou occupant une autre fonction similaire de direction ou de gestion. Le terme "membre" regroupe les simples membres. Précisons que dans les petites associations notamment, les rôles effectivement assumés par les individus peuvent ne pas correspondre formellement à leur statut sur le papier. 
du texte, sauf mention particulière, le terme "association" au sens générique, regroupant les deux formes juridiques d'action collective.

Les entretiens avec les acteurs associatifs ont été complétés par d'autres sources d'information, telles que la documentation interne aux associations (statuts, rapports d'activité, archives, bulletins d'information ${ }^{543} \ldots$ - inégalement riches et disponibles selon les associations) et leurs sites internet. À cela s'ajoutent des articles issus de recherches ciblées dans la presse quotidienne régionale et locale (Le Matin, 24 Heures, Tribune de Genève, Journal de Genève, Le Nouvelliste, L'Express...). Enfin, deux entretiens ont été réalisés avec des responsables de Swisstransplant pour mieux connaître la position actuelle et passée de la Fondation nationale suisse vis-à-vis des associations de patients.

Dans les chapitres qui suivent, nous nous intéressons à deux questionnements centraux pour comprendre le rôle des associations vis-à-vis du don d'organes comme problème public. Il s'agit premièrement de s'interroger sur la manière dont les acteurs associatifs se représentent la problématique du don d'organes et en particulier la pénurie d’organes. Rappelons que la définition de la situation constitue une dimension primordiale de la mobilisation profane. Les cadres d'interprétation mettent en jeu un contenu cognitif (puisqu'il s'agit de qualifier et de cerner ce dont il est question), et normatif ou moral, qui consiste à dénoncer une situation anormale, ou à réaffirmer des valeurs fondamentales et des comportements jugés désirables ${ }^{544}$. En nous référant à l'idée que la perception d'un problème implique notamment des phénomènes d'attributions de causalité et d'imputations de responsabilité, ainsi que des propositions de solutions ${ }^{545}$, il s'agira de décrire les cadres d'interprétation du don d'organes en Suisse qui sont à l'œuvre dans l'espace associatif romand.

543 Nous avons principalement consulté les numéros accessibles en ligne du Bulletin des As de Cœur et du Bulletin de l'AST (30 numéros de 2002 à 2015), du Journal de la SSPIR (7 numéros de 2012 à 2015), et de Swisstransplant News (devenu Swisstransplant Magazine) de 2008 à 2015.

544 Gusfield 2009.

545 Cefaï 1996. 
À cet égard, le problème de la pénurie d'organes, qualifiée de "catastrophique », "lamentable» ou "pire qu'avant ", fait l'objet d'un consensus fort dans notre corpus d'entretiens. Les interviewés évoquent une situation de crise ou d'urgence, expriment des réactions de découragement ou d'indignation, ou sont particulièrement critiques et alarmistes face aux conséquences fatales de la pénurie pour nombre de patients. Ils dénoncent la stagnation peu flatteuse du nombre de donneurs qui place la Suisse en bas de classement des pays européens ${ }^{546}$. Au-delà de l'aspect quantitatif, la pénurie d'organes est le symptôme d'un questionnement sur les causes du problème et les solutions préconisées. Pour les acteurs associatifs, le don d'organes en Suisse apparaît ainsi comme un triple problème: un problème politique et législatif, un problème hospitalier et un problème d'information de la population ${ }^{547}$. Les chapitres 7 et 8 examineront les tenants et aboutissants de ces trois cadres principaux de perception de la situation du don d'organes et montreront dans quelle mesure l'espace associatif partage une vision commune ou s'il est porteur de conceptions plurielles, voire conflictuelles.

Le second questionnement central consiste à analyser les domaines d'action privilégiés par les associations et leurs objectifs, en nous intéressant aux activités concrètement réalisées et au sens que les acteurs associatifs leur donnent. Le chapitre 9 décrira ainsi les différentes logiques d'action suivies par les associations. Il permettra aussi d'examiner dans quelle mesure les cadres de perception du problème des acteurs associatifs recoupent ou non les objectifs effectivement recherchés et affichés par les associations. À cet égard, la distance que l'on peut observer conduira à s'interroger sur la capacité de mobilisation de ces associations et leur influence sur le

546 Les interviewés font référence aux statistiques des années 2010-2014, montrant un taux de donneurs décédés par million d'habitants oscillant entre 12 et 14. En ligne: www.swisstransplant.org/fr/info-materiel/statistiques/chiffres-annuels

547 Ces trois cadres d'interprétation ne s'excluent pas; bon nombre d'interviewés situent leur discours sur les trois à la fois, mais il est fréquent que l'un des trois domine en termes de "cause" ou de "solution". Si certains interviewés développaient une vision bien définie et affirmée du problème en question, d'autres s'avéraient plus en peine d'exprimer leur perception des causes de la pénurie d'organes et des solutions envisageables. Néanmoins, tous à des degrés divers soulevaient la complexité du problème du don d'organes et relevaient l'absence de solution simple à la situation actuelle. 
don d'organes comme problème public. Si l'évolution du contexte institutionnel des dernières années joue un rôle indéniable, de même que la question des ressources, la compréhension de la dynamique de l'espace associatif du don d'organes requiert également, comme nous le montrerons, de prendre en compte les logiques individuelles d'engagement pour la cause. 


\title{
Chapitre 7
}

\section{Une cause négligée par les pouvoirs publics}

\author{
Raphaël Hammer
}

La grande majorité des acteurs associatifs considèrent que la situation perçue comme largement insatisfaisante du don d'organes relève de la responsabilité des pouvoirs publics. S'il reflète certes une société civile peu "donneuse ", le manque de greffons est avant tout dénoncé sous le prisme d'une action publique qui n'est pas à la hauteur des enjeux sociaux et sanitaires du don d'organes. Ce chapitre est consacré dans un premier temps aux différentes facettes de ce premier cadre d'interprétation d'un problème politique et législatif. Il s'agira de préciser la teneur des critiques à l'égard du manque d'engagement de l'État qui ciblent particulièrement la LTx, le consentement explicite et les campagnes officielles de communication. Nous examinerons également comment les acteurs associatifs se positionnent par rapport à différents modèles de régulation de l'échange d'organes. Nous aborderons ensuite le deuxième cadre d'interprétation du don d'organes en terme de problème hospitalier qui questionne l'organisation et les pratiques des professionnels impliqués dans la chaîne du don d'organes.

\subsection{Une cause peu reconnue}

La pénurie persistante d'organes est perçue comme le révélateur de l'absence de réelle volonté des autorités de s'engager pour le 
don d'organes, voire d'une certaine indifférence du politique ${ }^{548}$. Désenchantement et résignation accompagnent souvent les propos des interviewés qui fustigent le manque de soutien des pouvoirs publics à la cause: "l'État doit s'engager à fond et il faudrait faire plus de pub ", clame Christophe (FD, membre) qui dénonce, comme d'autres, la rareté des actions de sensibilisation auprès de la population. D'autres pointent du doigt l'inaction de l'État: «c'est le pouvoir politique qui est sourd»(Carlo, $A G$, membre); «le problème en Suisse, c'est plutôt au niveau gouvernement» (Stéphanie, AR, comité). Nombreux sont ceux qui estiment que le don d'organes n'est pas traité comme une priorité de santé publique: "soit on ne veut pas développer la transplantation et le don d'organes, et on reste sur ses positions et on se dit finalement que les gens soient malades, que les gens meurent en liste d'attente ce n'est pas notre problème, parfait! Moi je veux bien, mais au moins que l'on ait le courage de le dire... ou alors on dit: "oui, c'est une véritable cause nationale” » (Marc, FD, comité). La politique fédérale apparaît comme le niveau pertinent de lutte contre la pénurie et de promotion du don d'organes. La critique largement partagée d'un engagement insuffisant des pouvoirs publics se concrétise principalement à l'égard de la LTx qui a entériné la solution du consentement explicite comme principe régulateur de la circulation des organes. Les critiques de la loi portent également sur la politique de neutralité de l'État ${ }^{549}$ et la manière dont celle-ci s'est traduite dans la communication publique.

\section{La Loi fédérale sur la transplantation: une occasion manquée}

Dans leur grande majorité, les acteurs associatifs ont vu dans la LTx un mauvais signal pour la promotion du don d'organes et ont une opinion négative de l'évolution des débats sur la question

548 Précisons qu'une partie des entretiens ont été réalisés avant la publication du plan d'action national "Plus d'organes pour des transplantations", lancé par le Conseil fédéral en 2013, qui vise à atteindre un taux de 22 donneurs décédés par million d'habitants en 2021.

549 En ne prenant pas position sur le plan des valeurs, la LTx a été qualifiée de "cas typique de politique procédurale" en matière de question morale (Engeli et Varone 2011, 253). 
au cours des dernières années en Suisse. Le choix du consentement explicite, au lieu du consentement présumé550, est ressenti comme la preuve du manque d'engagement clair des autorités et d'une position excessivement timorée des politiques: "on sent que les politiques ont de la peine à se mouiller, c'est un petit peu entredeux [...] ils n'arrivent pas à faire bouger beaucoup de choses » (Laurent, FD, membre). Le choix du législateur, perçu comme de l'immobilisme coupable, suscite des réactions très virulentes chez plusieurs interviewés engagés depuis de nombreuses années dans le secteur associatif. Pour Pierre, la teneur de la loi est la conséquence d'un monde politique qui cherche à éviter les polémiques et les prises de risque par rapport à un sujet sensible: «On ne fait rien, on n'est pas une fois clair et net comme on fait en France où le gouvernement soutient plus activement le don d'organes, mais il faut être clair, prendre des risques, ici on est des gros chiards quoi!» (AR, comité). Si pour quelques interviewés, la LTx reflète - à mauvais escient - la culture consensuelle du monde politique helvétique, elle est surtout vue comme le signe que les autorités n'ont pas pris la mesure de la réalité tangible du problème du don d'organes. Pour de nombreux acteurs associatifs, l'élaboration d'une loi fédérale sur la transplantation aurait dû être une opportunité pour les autorités publiques de mener une politique plus active et en faveur du don d'organes, via l'introduction du consentement présumé. Marc est l'un de ceux qui expriment le mieux ce sentiment largement partagé que la LTx a été une occasion ratée pour mener une politique digne de ce nom en faveur du don d'organes: "Une loi était prévue au départ pour encourager, pour augmenter le nombre de donneurs, cette loi a été ensuite malaxée, triturée [...] et finalement dans le va-et-vient des deux chambres [elle] a été vidée de sa substance en quelques mois [...] [le projet de loi] a été limé, mais à la lime fine

550 Rappelons que le consentement présumé signifie que les médecins peuvent prélever les organes d'une personne décédée si celle-ci n'a pas exprimé son opposition de son vivant. Le consentement explicite (ou "au sens large") implique qu'un prélèvement d'organes ne peut être réalisé que si le défunt avait indiqué explicitement son consentement (en signant une carte de donneur par exemple). Si la personne décédée ne s'était pas exprimée, les proches peuvent autoriser le prélèvement. 
si je puis dire! Et au bout du compte il ne reste rien de cette loi!» (FD, comité).

Au-delà du sentiment d'une régression sociale qu'expriment certains, c'est celui d'une stagnation des dons d'organes qui prédomine, voire d'une aggravation de la pénurie. Les attentes déçues par rapport au choix final du législateur s'accompagnent de frustration chez plusieurs interviewés: "cette loi, quand ils en ont parlé au début, cela devait être le coup de pouce [...] je ne sais pas si elle ne l'a pas dégradée plutôt que l'améliorer [...] cela devait donner un petit coup de pouce au don finalement cette loi, mais on a reculé » (Pierre, AR, comité). D'autres soulignent que le choix du consentement explicite contribue à la pénurie en n'offrant aucune solution à la difficulté concrète dans laquelle se trouvent les personnes confrontées à un possible don d'organes: "la position fédérale, elle est ni blanc ni noir [...] [le principe d'autodétermination] c'est bien mais ça aide pas à prendre position dans les familles» (Pascal, AR, membre).

Quelques acteurs associatifs sont plus modérés dans leurs critiques et relèvent malgré tout des points positifs dans la LTx: la gestion nationale des organes (et non plus par centre hospitalier), une visibilité accrue de la question du don d'organes dans le débat public et les médias, ou encore l'existence même d'une loi fédérale en lieu et place d'une série de lois cantonales disparates. Néanmoins, en résumé, la loi est très largement perçue comme un problème à double titre. Premièrement parce qu'elle n'est pas en mesure d'améliorer significativement la situation comptable du don d'organes. Deuxièmement parce qu'elle révèle l'absence de volonté de l'État de s'impliquer véritablement pour la cause et d'engager une politique active. La vigueur des critiques à l'égard de la LTx et du consentement explicite s'explique aussi largement par les espoirs - déçus - que l'élaboration d'une loi fédérale avait nourris. Il faut en effet rappeler que l'option du consentement présumé était appliquée dans plusieurs cantons avant 2007, qu'elle avait été envisagée et discutée dans les phases préliminaires du processus législatif ${ }^{551}$, et enfin qu'elle est en vigueur dans des pays proches, à l'instar de la 
France ou de l'Espagne, qui affichent des taux de donneurs deux à trois fois supérieurs à celui de la Suisse. Ces éléments expliquent probablement qu'une majorité d'acteurs associatifs condamnent l'option retenue par le législateur. Il convient alors d'examiner leurs arguments en faveur du consentement présumé, bien souvent assimilé dans les discours publics à une mesure qui contribue à l'augmentation des dons d'organes.

\section{Les vertus du consentement présumé}

Contrairement à ce que l'on aurait pu attendre, l'argument du nombre de donneurs stricto sensu n'est pas central dans les propos des acteurs associatifs. Rares sont ceux qui présentent le consentement présumé comme une véritable solution au problème de la pénurie. La majorité des interviewés favorables restent très prudents quant à son impact direct sur l'augmentation de l'offre d'organes: "après avoir parlé avec beaucoup de gens qui travaillent chez Swisstransplant, qui travaillent dans les soins intensifs [...] on a appris que cela ne change pas beaucoup [dès lors que l'équipe soignante consulte de toute façon les proches du donneur potentiel] » (Gilles, AG, comité). Les interviewés soulignent que le consentement présumé à lui tout seul ne peut guère avoir d'influence s'il n'est pas accompagné d'autres mesures sur le plan de l'information du public et de l'organisation du travail hospitalier (voir 7.3).

Parmi les principaux arguments exprimés, le consentement présumé est perçu comme un moyen d'inciter à la prise de position de chacun par le renversement de la charge d'expression de sa volonté. Plusieurs interviewés soulignent que ce n'est pas à ceux qui sont disposés à faire un geste noble de subir le poids d'une démarche. Autrement dit, le consentement présumé fait peser l'effort de se faire entendre sur ceux qui ne veulent pas participer au système de solidarité, c'est-à-dire qui refusent d'être donneurs («opt-out system»): "ça change la perspective parce que si vous ne voulez pas être donneur [...] vous devez aller annoncer que vous ne voulez pas être donneur»(Suzanne, AG, membre). Cette inversion de la 
démarche apparaît comme plus cohérente avec la reconnaissance d'une valeur plus élevée du statut de donneur que de non-donneur.

Outre cette valeur morale, le régime du consentement présumé aurait aussi un avantage pratique. Dès lors qu'une majorité de citoyens ne fait pas spontanément l'effort de s'informer et de réfléchir à la question - "penser que les gens qui ne sont pas concernés vraiment vont prendre la peine d'aller trouver une carte de donneur et informer ses proches [...] on rêve ", affirme Nadia (FD, comité) -, cette option serait une incitation forte à ce que chacun prenne clairement position sur le don d'organes. En outre, le consentement présumé pourrait favoriser le débat social et la communication au quotidien sur le devenir de son corps après la mort: "ça aurait aussi le mérite de susciter beaucoup la discussion dans les familles et la prise de position au sein des familles plus, d'être plus proactif à quelque part...» (Pascal, AR, membre $)^{552}$. En donnant ainsi une meilleure visibilité à cet enjeu dans la société, le travail des soignants serait facilité: "en Suisse, il y a beaucoup de gens qui sont surpris qu'on pose la question [du don d'organes] [...] déjà pour le médecin j'imagine que l'approche est plus facile» (Laurent, FD, membre).

Au-delà des aspects concrets, l'argument principal en faveur du consentement présumé est de nature symbolique. Pour beaucoup d'acteurs associatifs, un tel changement législatif serait (et aurait été) un signal clair et fort que l'État s'engage véritablement pour la cause en cherchant à améliorer la situation. Indépendamment de sa propension à augmenter le nombre de donneurs et à réduire celui des patients sur liste d'attente, le consentement présumé a donc aussi une valeur politique de la reconnaissance du don d'organes comme problème public et comme cause légitime.

\section{Les campagnes de l'OFSP}

Les campagnes de l'OFSP cristallisent les critiques à l'égard de l'action publique et du manque d'engagement politique. La dénonciation de la prudence excessive des messages officiels s'illustre tout

552 Selon certains travaux, les efforts d'information sur le don d'organes par les autorités auprès de la population seraient au contraire plus nourris dans les pays pratiquant le consentement explicite (Pfaller et al. 2018). 
particulièrement à propos d'une brochure distribuée à l'ensemble de la population quelque temps avant le début de notre enquête. Nous avons ainsi recueilli le point de vue des acteurs associatifs à propos du contenu de cette brochure intitulée «Informations sur le don d'organes, de tissus et de cellules en cas de décès. Tous ont raison: ils expriment leur volonté ${ }^{553}$. " Les messages délivrés dans cette brochure, qui s'inscrivait dans une stratégie de l'OFSP comportant d'autres supports de communication, tels que des affiches, des bannières internet et des séquences audiovisuelles analysées par Merminod ${ }^{554}$, reflètent les contraintes du mandat d'information posées par l'État ${ }^{555}$. En effet, la mission de l'OFSP n'est pas d'inciter les citoyens au don d'organes mais d'informer «le public régulièrement sur les questions liées à la médecine de transplantation» et de «donner à chacun la possibilité d'exprimer sa volonté concernant le don d'organes, de tissus ou de cellules en toute connaissance de cause ${ }^{556}$ ».

\section{"Tous ont raison "... ou presque}

Le reproche principal adressé au contenu de la brochure concerne la neutralité revendiquée de cette action de communication publique. La majorité des interviewés s'insurgent contre le fait que la brochure n'affiche pas plus clairement une position en faveur du don d'organes, et regrettent que le message soit faible, mou, voire illisible ou obscur pour les destinataires.

553 Il s'agit d'une brochure de douze pages en couleurs donnant des informations factuelles portant par exemple sur les conditions légales de la transplantation, les mesures médicales préliminaires et la carte de donneur. En outre, la brochure contient une série de visages accompagnés de huit opinions pour ou contre le don d'organes, telles que: "Je suis plutôt pour le don d'organes et de tissus. Mais à vrai dire, je ne me suis pas encore vraiment posé la question", "Je suis pour le don d'organes. Quand ma nièce est morte, on a fait don de tout. Cinq vies ont ainsi pu être sauvées", ou "Je suis contre le don d'organes en cas de décès. Je fais beaucoup de dons sinon toute l'année. Mais mon corps il m'appartient, à moi."

554 Merminod 2013 et 2015.

555 Merminod relève que "d'une manière générale, les campagnes ont évolué d'une information sur le cadre juridique entourant la médecine de transplantation en 2007 à une incitation à l'expression de sa volonté à partir de 2008 " $(2013,135)$.

556 LTX, art. 61. 
Pour Gilles, l'OFSP outrepasse son mandat en introduisant dans l'espace public des messages relatifs à la perception subjective de la mort, défendant l'idée que celle-ci relève de la sphère privée. De son point de vue, le rôle de l'État n'est pas de communiquer sur le rapport individuel à la mort, encore moins d'exercer une influence, délibérément ou non, sur cette question ${ }^{557}$ : "la mort, c'est une discussion que l'on doit faire à la maison avec ses proches, ses amis, mais cela ne doit pas être une discussion officielle... ce que le bureau fédéral doit communiquer c'est: “voilà Mesdames et Messieurs, c'est une grande chance pour les personnes qui sont à la limite de la vie de recevoir un organe", point final! Le reste est quelque chose que l'on doit discuter dans le privé, ce n'est pas le devoir de l'OFSP de communiquer là-dessus" (Gilles, AG, comité). Comme d'autres acteurs associatifs l'affirment, le seul message légitime que l'État doit transmettre à la population est l'information sur le bénéfice thérapeutique que représente le don d'organes. Pour Gilles, la situation des malades en attente d'un organe (et leur statut de receveurs potentiels) doit être le point de départ de la réflexion de chaque citoyen. Dans cette perspective, la mention d'avis négatifs, notamment, vis-à-vis du don d'organes constitue un pas de trop et va à l'encontre de la neutralité dont se réclame l'OFSP.

Tout en soulignant l'importance du principe général de l'autodétermination, nombre d'interviewés dénoncent certains messages équivoques ou ambivalents, à l'instar du «témoignage» figurant en première page de la brochure: «Il faut être clair: "Je suis pour le don d'organe", on n'a pas à tergiverser... ou on est pour ou on est contre, mais on ne va pas dire: "Je suis plutôt pour le don d'organes et de tissus, mais à vrai dire, je ne me suis pas encore [vraiment] posé la question”... il faut être décisif dans un truc [comme ça]» (Suzanne,

557 Trois opinions contenues dans la brochure portent sur la question de la mort. La première, en faveur du don, réfère à la mort cérébrale: "Je suis pour le don d'organes et de tissus. Quand mon cerveau est mort, la vie est finie." Les deux autres opinions sont en défaveur du don de ses organes. L'une évoque la réticence viscérale à l'idée du prélèvement: "Je suis contre le don d'organes. Je ne supporte pas l'idée qu'on me découpe en morceaux quand je serai morte", et l'autre fait allusion à une conception "intégraliste" de la mort (Herpin et Paterson 2000): "Moi je suis contre le don d'organes en cas de décès. Je suis convaincu que chaque partie de mon corps a une âme." 
AG, membre). Le manque de lisibilité de l'intention du message général de la campagne est relevé à plusieurs reprises, comme cette présidente qui critique l'évocation d'une multitude d'opinions possibles sur le don d'organes: "Ils ont voulu prendre vraiment un panel large, alors justement, en mettant des jeunes, des vieux, et tous les cas, “je suis contre”, “je suis pour”, “je doute”... je trouve que c'est un peu flou, ce n'est pas clair» (Julie, AR, comité). Il en va de même pour cette interviewée, à la fois dubitative et très virulente à l'égard de l'OFSP: «ça c'était nul! cela m'a énervé ces campagnes [...] on [les autorités] fait une campagne, une information sur le don d'organes et puis on entend des gens qui disent "moi je donne", "moi je ne donne pas" [...] moi je trouve que ces "non, je ne donne pas"... alors que c'est une information sur le don d'organes, mais il faut positiver... je dois dire que je n'ai pas vraiment compris » (Brigitte, AR, comité).

Dans l'ensemble, la mention d'avis «contre» le don d'organes est perçue comme foncièrement contradictoire avec l'idée d'une information neutre, quand bien même les avis "pour» le don d'organes sont aussi représentés. Pour la plupart des acteurs associatifs, cette pluralité d'opinions et d'arguments vis-à-vis de la transplantation ne contribue guère à la réflexion de chacun ou au débat social. Aux yeux de plusieurs interviewés, la brochure de l'OFSP est un pavé dans la mare, dans la mesure où elle attribue à l'opposition individuelle au don d'organes une valeur quasiment identique à celle du «oui ». Une double critique est donc énoncée vis-à-vis des pouvoirs publics: le refus de prendre position en faveur du don d'organes (s'abstenir de se prononcer «pour») et la légitimation du non (dire explicitement qu'on peut être contre le don d'organes).

\section{Ni pour, ni neutre}

Plusieurs acteurs associatifs émettent une troisième critique plus virulente encore à l'égard de la campagne officielle, y voyant non une information maladroite et un message peu lisible, mais une action qui en fin de compte «met davantage l'accent sur le non-don que sur le don» (Pascal, AR, membre). Les vignettes défavorables 
au don sont jugées plus convaincantes que les vignettes favorables: «c'est même à la limite d'être contre » (Nadia, FD, comité). La mise en exergue d'opinions négatives est susceptible de biaiser le principe d'autodétermination en orientant le choix du citoyen davantage vers le «contre» que vers le «pour». Léo, qui n'est pourtant pas partisan d'une information centrée uniquement sur les aspects positifs de la transplantation (voir plus loin), déplore un déséquilibre manifeste: «j'ai trouvé qu'ils insistaient beaucoup sur les personnes qui étaient contre» (AG, membre). Dans ces conditions, il doute fortement que ce type de communication puisse retenir l'attention des citoyens hésitants ou indécis: "[des personnes] qui sont moins sensibilisées, je ne sais pas comment elles réagissaient avec ça, je ne suis pas sûr que cela ait attiré beaucoup de monde dans le don d'organes, parce que je l'avais trouvée plutôt négative. »

Les propos de Charles illustrent parfaitement le sentiment d'indignation d'une partie des interviewés vis-à-vis d'une action publique qui, à leurs yeux, aboutit, sous couvert de neutralité d'information, à un renversement des valeurs sur le don d'organes. Comme d'autres l'évoquent, compte tenu des enjeux majeurs de santé en présence, la superposition des " oui » et des «non» ne peut se réclamer d'une démarche neutre et revient à légitimer le «non» au détriment du «oui». Suggérer que les deux positions sont comparables entraîne une dévalorisation inacceptable des arguments en faveur du don d'organes. Une telle mise en équivalence morale est vécue comme intolérable lorsqu'à titre personnel on doit sa vie à un "oui »: "cette espèce de pub multi-choice! C'est terrible! En tout cas, moi comme transplanté, ça m'agace» (Charles, AG, membre). Autrement dit, la portée (symbolique ou réelle) d'un «non » est trop forte pour avoir la même valeur qu'un «oui». Ce «nivellement» moral attribué à la brochure revient à entériner socialement et éthiquement un individualisme forcené mal placé face à la (sur)vie d'une personne: «... deux positions, présentées comme équivalentes et qui instaurent quelque chose de complètement monstrueux, le moi comme un absolu» (Charles, AG, membre). Pour cet interviewé, le don d'organes constitue précisément un sujet de société "où le moi est haïssable, où le je n'est pas absolu [...], où on n'est pas seul et où 
le choix solitaire n'a pas de sens comme phénomène public». De son point de vue, il est de la responsabilité de l'État de faire en sorte que les oppositions n'aient pas socialement la même valeur morale que les positions favorables au don. Et d'ajouter que cette équivalence trahit la distribution des attitudes dans la population: «En mettant à égalité, l'OFSP fait une saloperie, parce qu'elle donne trop d'audience à des narcissico-égoïstico-machin-chose! [...] statistiquement, on est une belle majorité pour le don! On n'a pas le droit de faire cette pub d'équivalence!»(Charles, AG, membre).

\section{Un contre-discours malvenu}

Si les interviewés dénoncent le contenu de la brochure de l'OFSP et, de manière sous-jacente, l'état d'esprit des pouvoirs publics, ils critiquent aussi les effets de tels messages sur les dispositions de la population vis-à-vis du don d'organes. La légitimité du «non » au don d'organes, produite par la mention explicite d'opinions négatives, est de nature à alimenter les oppositions de la population et les refus de prélèvement: "Cette pub de l'OFSP, c'est de la cochonnerie, c'est contre le don d'organes [...] on sait [qu']après cette campagne de l'OFSP le vœu de la population a changé, c'est plutôt maintenant contre le don d'organes, il y a beaucoup de gens qui disent non»(Gilles, AG, comité). D’autres acteurs associatifs mettent en avant les effets parasites de la neutralité des campagnes de l'OFSP sur leurs propres activités au contact du public (voir chapitre 9). Aux yeux de Pierre notamment, le message de la brochure dessert leurs démarches d'information et de sensibilisation, suscitant auprès de la population un sentiment de perplexité ou de confusion sur l'attitude à adopter vis-à-vis du don d'organes: "Ils viennent nous saboter avec leurs réclames là, moi je n'ai vraiment pas trouvé cela bon!» (AR, comité). Plusieurs interviewés évoquent un conflit de points de vue qui s'exerce à leur détriment dans la mesure où les messages en faveur du don d'organes deviendraient moins audibles et moins crédibles. Pour certains, la brochure est contre-productive car elle risque d'alimenter des croyances erronées sur le prélèvement d'organes. Évoquant les efforts contrecarrés de 
Swisstransplant pour sensibiliser la population à la cause du don d'organes, Daniel mentionne l'une des vignettes: «Ils les sabotent d'une certaine manière par l'édition d'une brochure [qui] est [...] au mieux catastrophique, au pire mensongère [...] une personne dit: "moi je ne veux pas donner mes organes, parce que je ne veux pas que l'on me coupe en morceaux", quand on connaît [le travail] fait par les gens qui prennent l'hélico, au moment d'un prélèvement du don d'organes, et puis qui vont prélever un foie, c'est trois heures et demie d'opération... en aucun cas, les gens arrivent avec le scalpel et puis comme des vautours... c'est d'une maladresse terrible sur le plan psychologique, parce que les gens qui lisent [vont comprendre] “j'ai raison d'avoir peur, puisqu'ils l'écrivent [...] c'est comme cela que cela se passe, puisque le témoignage est dans cette brochure" " (AG, comité).

Compte tenu des attentes fortes qu'il y avait à l'égard du processus politique de la nouvelle loi, cette campagne de l'OFSP, par son contenu et sa neutralité, est perçue comme une régression sur le plan moral et social, voire comme une trahison des autorités. Le ressentiment de certains acteurs associatifs tient aussi au fait que jamais autant de ressources financières n'avaient été engagées dans des actions de communication publique, et que les opinions négatives à l'égard du don d'organes n'avaient jamais reçu une telle publicité officielle. Plus encore, la brochure est perçue par certains comme un contre-discours susceptible de saper le travail d'information mené par les associations.

En résumé, à l'exception de quelques interviewés, la critique des campagnes de l'OFSP est largement dominante dans notre corpus et cible leur coût élevé, leur frilosité, leur manque de pertinence et d'efficacité, ou encore leurs méfaits sur la disposition au don du public. Rappelons que certaines critiques visant le contenu des messages l'OFSP sont formellement inadéquates dans la mesure où la neutralité de l'information publique n'est pas un choix de sa part, mais bien une orientation décidée par le législateur à laquelle l'OFSP est soumise. Notons que la défiance émanait aussi d'autres acteurs, 
plus institutionnels, à l'instar de Trix Heberlein, alors présidente de Swisstransplant, pour qui «les campagnes de l'OFSP diffusent trop d'images négatives. Cela est contre-productif et aggrave l'insécurité au sein de la population ${ }^{\mathbf{5 5 8}}$.» Pour de nombreux acteurs associatifs, l'évidence morale et de santé publique voudrait que la communication officielle poursuive des objectifs de promotion ou de soutien du don d'organes, dès lors que des vies sont en jeu. La neutralité de l'information et de l'État est le point qui suscite le plus d'incompréhensions et qui fâche le plus. Elle est souvent assimilée à une absence de politique véritable, reflétant le non-engagement des autorités, si ce n'est leur incapacité à prendre le problème du don d'organes à bras-le-corps: "la neutralité, c'est le RIEN dans ce domaine, on ne peut pas être neutre [...] soit on fait quelque chose en faveur, soit on lutte en défaveur! Mais on ne peut pas être neutre! Et là dans cette question-là, alors plus neutre que cela, c'est juste pas possible, résultat des courses cela ne sert à rien! [...] la voie officielle, c'est du RIEN» (Marc, FD, comité).

\subsection{Quel modèle d'organisation pour l'échange d'organes?}

L'autodétermination et le don librement consenti de ses organes, qui constituent des valeurs cardinales pour les acteurs associatifs, peuvent se concrétiser de manières différentes et donner lieu à des conceptions contrastées de la régulation du don d'organes. Dans les entretiens, outre l'opposition entre les consentements présumé et explicite, nous avons cherché à connaître le positionnement des acteurs associatifs sur des modèles alternatifs de circulation des organes, généralement envisagés comme des mesures susceptibles de réduire la pénurie ${ }^{559}$. Il s'agit des modèles du registre et du choix contraint, relativement proches dans leurs principes, qui ont été occasionnellement mis à l'agenda politique et médiatique au cours

558 AST News, $n^{\circ}$ 41, mai 2013, 9.

559 La pénurie chronique d'organes au niveau international a en effet suscité de nombreuses réflexions politiques, juridiques, éthiques et économiques sur des modèles alternatifs de régulation du don d'organes. Voir par exemple les travaux de Steiner 2010, Mader 2011, Veatch 2000. 
des dernières années en Suisse. Rappelons que la solution d'un registre de donneurs d'organes était la raison d'être des revendications portées par l'association Passez-le-Relais (voir chapitres 6 et 9). Nous avons également sondé les interviewés à propos de deux autres modèles théoriques, basés sur l'incitation économique et le principe de réciprocité ${ }^{560}$.

Par-delà la reconnaissance du principe de l'autodétermination, les principales divergences observées dans les entretiens quant à la ou les meilleures manières d'organiser le don d'organes font ressortir trois enjeux majeurs: le respect ou non de la volonté expresse du donneur potentiel, le pouvoir des proches, et l'obligation de se déterminer pour chaque citoyen. Dans une certaine mesure, ces lignes de tension dépassent ou transcendent l'opposition étroite entre les variantes légales du consentement (présumé ou explicite). La question des incitations économiques et du modèle de la réciprocité permet de compléter l'éventail des attitudes des acteurs associatifs sur la régulation du don d'organes.

\section{La volonté expresse du donneur et l'esprit du don}

L'option du consentement présumé recueille, on l'a vu, un assentiment assez fort parmi les acteurs associatifs, davantage pour sa portée sociale et symbolique que pour des motifs d'efficacité et d'augmentation des organes à greffer. Cette option suscite néanmoins aussi des critiques et des réserves.

Plusieurs interviewés ont ainsi affirmé avec force leur attachement à ce que les prélèvements d'organes ne soient pratiqués qu'à la condition que le donneur ait expressément fait part de son désir de donner ses organes après sa mort. Le régime du consentement explicite est alors perçu comme seul garant de la volonté réelle du donneur décédé exprimée préalablement. Dans cette optique, le consentement présumé - appliqué de manière stricte - est assimilé à un risque de dénaturation de l'acte de don. Il équivaut à un don contraint, loin de l'idéal du don librement choisi et décidé en toute

560 L'Iran est le seul pays disposant d'un marché légal et régulé du don d'organes entre vivants. Le modèle basé sur le principe de réciprocité est en vigueur dans quelques pays, dont Singapour. 
connaissance de cause; "cela rejoindrait presque à obliger les gens, enfin, même s'ils sont décédés, leur faire faire quelque chose qu'ils ne souhaitaient pas, et puis peut-être [qu']ils n'ont pas pris le temps [d'y réfléchir]» (Laura, AR, comité). De manière plus catégorique, Léo rejette le consentement présumé qui à ses yeux revient à pervertir la noblesse, la pureté du geste, voire à vider de son sens l'idée même de don; "c'est plus du don à ce moment [...] c'est une confiscation pour laquelle on ne peut que s'opposer... il n'y a plus l'esprit du tout du don d'organes. Donner, c'est prendre une décision et faire un geste, si c'est obligatoire ce n'est plus un don" (AG, membre). Le consentement présumé est ici assimilé à une forme de nationalisation des organes, à « une contribution à la santé ou je ne sais pas comment on peut le définir, et je crois que là il faut qu'on fasse attention » (Léo, AG, membre). Renoncer au consentement explicite signifierait prendre le risque de fausser l'esprit du don, et de réduire davantage le nombre de donneurs potentiels. Dans cette première ligne de tension, l'attachement à la volonté expresse du donneur à travers le principe du consentement explicite témoigne d'un désir de préserver l'authenticité du geste et de ne pas altérer la valeur du don véritable, forgée dans l'intention assumée de faire le bien ${ }^{561}$.

\section{La décision médicale de prélèvement: avec ou sans les proches?}

Une deuxième ligne de tension concerne le respect de la volonté exprimée par le donneur potentiel et le rôle des proches dans la décision médicale de procéder ou non au prélèvement du parent décédé. La question est ici de savoir si l'avis de la famille doit être pris en considération; les médecins doivent-ils renoncer au prélèvement si les proches s'opposent au choix exprimé par le défunt?

Plusieurs acteurs associatifs estiment que les médecins ne devraient pas tenir compte de l'avis des proches lorsque le défunt était un donneur déclaré, arguant du fait qu'il n'y a pas d'impératif moral qui les obligerait à obtenir systématiquement l'assentiment

561 Cette préoccupation à l'égard d'un don exprimé en toute conscience fait écho à un certain nombre de critiques formulées en France à l'encontre de l'usage du terme "don" dans un système fondé sur le consentement présumé (voir Introduction). 
de la famille pour le prélèvement. Au nom du respect du défunt, les proches ne devraient pas pouvoir contrarier le choix exprimé de son vivant par leur parent. Critiquant les usages en milieu hospitalier - l'opposition de la famille est respectée -, Bernard dénonce le pouvoir indu de cette dernière qui va à l'encontre de l'autonomie de la personne décédée: "C'est pas normal, pourquoi est-ce qu'on doit demander l'avis à la famille? La personne qui est convaincue d'un don d'organe, qui a rempli sa carte, bon après il n'y a plus de souci... ce n'est pas comme ça que le système doit fonctionner, on a le char à l'envers ici en Suisse [...] la famille, c'est pas son problème! Le problème c'est la personne qui décède! Voilà, c'est tout. C'est elle qui décide, pas la famille» (AG, comité). Certains font valoir que le rôle déterminant de l'avis des proches dans la décision médicale de prélever ou non contredit l'esprit de la loi. Par exemple, Julien s'insurge contre les pratiques du corps médical qui à ses yeux constituent une contradiction, voire une tromperie par rapport aux discours officiels sur l'importance de l'autodétermination de chaque citoyen, précisément afin d'éviter aux proches le fardeau de se prononcer à la place du défunt; "quelqu'un a la carte et la famille, le papa, la maman, les frères et sœurs disent "non"; je me demande pourquoi ils peuvent intervenir [...] on a toujours dit: "Prenez le temps de vous décider", etc., en réalité ce n'est pas le cas, moi j’ai trouvé que là on nous a menti, c'est désagréable [...] parce que chaque fois qu'on est confronté au problème... le médecin dit: "Oui mais il faut le consentement des proches" »(AR, comité).

Un autre argument justifiant le respect strict de la volonté du défunt réside dans le fait que le refus des proches ne résulte pas d'une opposition en soi au prélèvement mais de la forte charge émotionnelle de la situation. En d'autres termes, la famille à qui l'on vient d'annoncer la mort d'un être cher n'est pas en état de répondre valablement à la question du don qui leur est posée dans des circonstances dramatiques. En outre, la difficulté de comprendre et prendre conscience de la mort cérébrale ne permet pas une discussion sereine sur la question d'un don d'organes, sans compter que celle-ci représente une souffrance supplémentaire; "pour les proches c'est très difficile de prendre des décisions lorsque la personne est 
en mort cérébrale, parce que l'on n'a pas l'impression qu'elle est complètement morte vu que les fonctions vitales sont maintenues, non, pour moi c'est un massacre de demander à la famille, je trouve que c'est trop compliqué » (Christophe, FD, membre). Aborder le don d'organes avec les proches abasourdis par la terrible nouvelle de la mort d'un parent ne ferait qu'alourdir encore un peu plus leur détresse; "les gens sont complètement anéantis, parler du don d'organes... les gens ne sont pas prêts à parler de ça, et c'est normal, moi la première... je sais que moi je donnerai mes organes, mes enfants aussi mais peut-être que devant l'horreur de voir mes gamins se faire écraser par une bagnole [...] peut-être qu'au niveau émotionnel, je ne serai pas prête à supporter une autre information » (Murielle, FD, comité).

Plusieurs acteurs associatifs défendent néanmoins les pratiques en vigueur des médecins qui renoncent au prélèvement si la famille s'y oppose. Dans notre corpus, la justification de l'implication des proches dans la décision médicale relève de deux logiques différentes. La première renvoie à une conception "relationnelle ${ }^{562}$ " du geste de donner qu'expose notamment Sylvie (AG, membre). Le fait de consulter la famille lui paraît normal et l'idée qu'elle ne soit pas impliquée heurte ses convictions: "Je trouve qu'il faut demander à la famille, pour moi c'est essentiel quand même, sinon où va-t-on? » À ses yeux, le geste du don doit autant que possible rester à l'abri des lois et des protocoles. La nécessité de préserver le caractère fondamentalement humain du don légitime le fait de ne pas exclure les proches sur la seule base d'une disposition formelle. Compte tenu du contexte émotionnel, symbolique et relationnel particulier du prélèvement d'organes, consulter la famille et suivre son avis sont des démarches naturelles, alors que la tenir à l'écart reviendrait à dénaturer le don. Réglementer la discussion sur le don en situation est perçu comme un artifice contraire à l'esprit du don et à sa complexité. Le désaccord éventuel des proches fait en quelque sorte partie intégrante de cette complexité qu'il ne faut pas chercher à simplifier ou à évacuer par des procédures, quand bien même

562 Steiner 2004. 
celles-ci seraient liées à des principes éthiques forts (l'autodétermination et le respect strict de la volonté du donneur par exemple). De son point de vue, donner ses organes ne doit pas être une obligation légale mais le fruit d'une décision volontaire: «Pour moi, ce n'est pas un devoir de donner [...] chacun doit être libre de ce qu'il veut, on a déjà assez légiféré, je suis contre toutes ces lois [...] laissons notre libre arbitre» (Sylvie, AG, membre).

La deuxième logique justifiant l'implication des proches dans la décision du prélèvement tient à la nécessité de conserver un climat social consensuel autour de la médecine de transplantation. Ainsi, pour Georges (AR, comité) qui soutient une application souple du consentement présumé, les équipes soignantes ont raison de demander l'avis aux familles afin d'éviter toute situation de conflit. Le respect de la position des proches, même s'il va à l'encontre du choix du défunt, est nécessaire pour préserver une bonne image publique du don d'organes. Cette image est fragile, exposée aux risques de scandale, aux plaintes et aux soupçons de manquement éthique; "si quelqu'un a dit "oui" et puis que la femme dit "non", c'est difficile de... parce qu'après il y a tout le risque: "oui ils ont arrêté la machine parce qu'ils voulaient prendre les organes” [...] ça peut faire de très grands dégâts dans le domaine du don d'organes s'il y a un procès, si quelqu'un accuse l'hôpital, même si ce n'est pas vrai, le fait d'accuser d'avoir débranché la machine pour pouvoir donner les organes, à mon avis... donc il faut que la famille donne son consentement" (Léo, AG, membre). L'accord des familles pour le prélèvement est ici moins défendu pour des raisons éthiques ou psychologiques que pour des motifs stratégiques: préserver la confiance de la population en évitant toute mauvaise publicité en lien avec le don d'organes, ses acteurs et ses pratiques.

Enfin, d'autres interviewés expriment une position plus nuancée sur la place de la famille. Pascal suggère que les proches soient partie prenante de la discussion autour du don, sans pour autant leur attribuer un poids dans la décision: "Ce serait dommage qu'ils aient la possibilité de bloquer, ce devrait être plus de l'ordre de l'information et du rappel de leur dire: "Voilà votre fils ou votre conjoint, votre père a sa carte, il est pour, vous savez qu'il ne s'est 
jamais opposé”... je suis assez [favorable] pour ne pas prélever sans les informer, sans les contacter, c'est là que cela fait beaucoup de sens d'avoir des équipes sur place pour rencontrer les familles et les soutenir dans ce moment-là» (AR, membre). Cette position médiane - impliquer la famille dans la démarche du don sans lui donner un rôle décisionnel - cherche à concilier plutôt qu'à opposer deux considérations éthiques majeures : le respect de la famille et le respect de la volonté du donneur. La position hésitante de Brigitte, partagée entre l'application stricte de la volonté du défunt et la prise en compte de l'entourage, montre bien que le rôle des proches reste une question épineuse; "qu'en dernier lieu [les médecins] demandent encore à la famille... est-ce que cela doit être fait ? c'est une grande question... par respect du défunt, pour la famille, pour les proches... je ne sais pas, ce n'est pas une question facile» (AR, comité).

\section{Obligation ou liberté de se prononcer}

La troisième ligne de tension concerne l'obligation de se prononcer sur le don d'organes. Tout un chacun devrait-il être contraint de se déterminer officiellement sur cette question, ou rester libre de ne pas s'interroger sur cet enjeu? Cette question est au cœur des modèles du registre et du choix contraint (mandatory choice).

Pour nombre d'acteurs associatifs, l'inscription systématique du statut de donneur ou non-donneur dans un document officiel (carte d'assurance maladie, carte d'identité, permis de conduire par exemple) est apparue comme une solution possible à la pénurie d'organes. Le caractère systématique de l'enregistrement de la position des individus permettrait, à leurs yeux, de surmonter les limites pratiques du système actuel de la carte de donneur (non obligatoire). Cet aspect n'est pas anecdotique dès lors que, si l'on en croit les sondages, une proportion importante de la population se dit en faveur du don d'organes sans toutefois avoir signé une carte ${ }^{563}$. Pour certains interviewés, la mise en place d'un registre de non-donneurs

563 Un sondage réalisé en 2015 indique que $81 \%$ des enquêtés étaient prêts à donner leurs organes après leur mort et que $53 \%$ avaient exprimé leur volonté, en ayant informé leurs proches ou signé une carte de donneur (Swisstransplant, communiqué de presse, 7 septembre 2015). 
dans un régime de consentement présumé constituerait un progrès dès lors que les donneurs potentiels le deviendraient automatiquement officiellement. Un autre avantage relevé par plusieurs acteurs associatifs est de forcer les individus à réfléchir à la question du don d'organes et à déterminer leur position personnelle, ce qui réduirait considérablement la part des indécis et de ceux qui ne font pas l'effort de se prononcer.

La contrainte de l'enregistrement du choix des individus peut être absolue ou relative. Dans le premier cas, l'individu doit se prononcer pour ou contre sans autre alternative, dans le second cas, il aurait la possibilité de choisir une position indéterminée. Cette option minimale est privilégiée par Léo: "maintenant, la prochaine étape si on veut vraiment aller plus loin, c'est de trouver un moyen de demander aux gens leur choix, s'ils ont une décision en respectant le fait qu'ils n'en aient pas » (AG, membre). Considérant que le don d'organes est devenu une nécessité sociale autant qu'une évidence politique, plusieurs acteurs associatifs plaident pour la première option (contrainte absolue). Tout un chacun aujourd'hui devrait se poser la question du don et se déterminer sur le fond, positivement ou négativement; "c'est une décision que toute personne doit prendre une fois dans sa vie et consacrer dix minutes, il n'y a pas besoin de longtemps réfléchir [...] la décision doit être prise... il faut arriver à un certain moment à forcer la main aux personnes en disant: “Mais il n'y a que deux solutions, soit oui, soit non” » (Bernard, AG, comité). Enfin, plusieurs interviewés avancent que l'enregistrement systématique du statut de donneur ou non de chaque citoyen sur un document officiel ou dans une base de données permettrait aux professionnels de santé de savoir rapidement si tel ou tel patient en état de mort cérébrale avait exprimé la volonté de donner ses organes, évitant ainsi les incertitudes liées au système de la carte de donneur «papier ${ }^{564}$ ».

564 Swisstransplant a lancé en 2014 la carte de donneur au format numérique sur smartphones, qui peut être lue automatiquement par le personnel médical lorsqu'un patient est pris en charge dans un service d'urgences. 


\section{Incitations économiques}

L'option des incitations économiques pour augmenter le nombre de donneurs d'organes potentiels constitue un sujet classique de la littérature éthique et économique ${ }^{565}$ et un thème débattu dans les milieux professionnels et politiques aux États-Unis notamment ${ }^{566}$. Dans notre corpus, le bien-fondé des incitations économiques au don d'organes (telles qu'une réduction de primes d'assurance maladie ou un rabais d'impôt) pour lutter contre la pénurie est partagé.

Les oppositions exprimées ne sont guère indexées sur des critères d'efficience (augmentation potentielle du nombre de donneurs) mais sur des critères moraux. L'argument principal des interviewés consiste dans l'incompatibilité de principe entre l'esprit du don - la gratuité - et la sphère économique, connotée négativement, mettant en jeu deux registres d'action difficilement conciliables, si ce n'est irréductibles; "c'est dangereux ça [...] c'est toujours un peu délicat d'arriver dans le côté financier ou comme ça je trouve, dans ces domaines [...] déjà dans la santé ce n'est pas évident, y a des trucs quand même qui ont tendance un peu à déraper» (Laurent, FD, membre). Cette dimension financière du don d'organes est parfois associée aux dérives de la marchandisation des corps; "c'est curieux ça, dans le don, il y a donation, c'est justement le don de soi, il y a la notion de gratuité, cela me fait presque penser au trafic d'organes » (Christophe, FD, membre). Plusieurs interviewés ne voient pas de justification à ce que la disposition au don fasse l'objet d'une transaction économique ou matérielle; «j'estime qu'il n'y a pas de raison de rémunérer quelqu'un qui simplement dit "oui, quand je serai mort je donne mon...”, si on arrive à garder ça, c'est positif » (Laurent, FD, membre). À leurs yeux, le don d'organes doit rester en dehors de la sphère marchande, sa valeur tenant justement à son extraterritorialité économique. Ils insistent sur le fait que le don d'organes doit rester un acte foncièrement désintéressé. En tant que geste altruiste,

565 Voir par exemple Steiner 2010.

566 Cette option ne doit pas être confondue avec la vente légale d'organes qui, dans notre corpus, suscite des oppositions catégoriques. Les entretiens montrent qu'il y a un consensus fort sur le caractère inconcevable et inacceptable d'un système marchand officiel d'échanges d'organes. 
il ne peut s'accommoder d'une quelconque rétribution; " eh bien, non! non, ce n'est pas vénal... c'est un acte d'amour, on donne les organes parce que tout cela ne nous sert plus à rien quand on est mort et parce que cela peut sauver des vies» (Julie, AR, comité).

Alors qu'une partie des acteurs associatifs n'entrent pas en matière sur la question par principe, d'autres s'avèrent plutôt réceptifs à la mise en place éventuelle de mesures incitatives économiques afin d'augmenter le nombre de donneurs déclarés; "cela ne me choque pas particulièrement", indique Carine (FD, comité). Un autre interviewé est a priori favorable à "une compensation financière » ou "une récompense » (matérielle ou symbolique) pour les donneurs potentiels, qui à ce titre "participent à la solidarité nationale». Il est cependant rare que les interviewés ouverts aux incitations économiques comme mécanisme de la régulation du don d'organes n'assortissent pas leurs propos de mises en garde. Ainsi, Didier, pour qui «cela pourrait être une solution ", pointe les risques de dérive: «il faudrait éviter que cela devienne dans l'esprit des gens, que cela devienne payant, un don doit rester un don, cela ne doit pas être l'attrait» (AR, comité). On retrouve, comme précédemment, les notions de gratuité et de désintéressement comme critères fondamentaux d'un don authentique, seul digne d'être pratiqué et défendu.

\section{Le principe de réciprocité:}

\section{altruisme intéressé ou générosité récompensée?}

Le modèle de régulation des organes basé sur le principe de réciprocité (proche de la formule "club») introduit la distinction entre deux catégories de malades en attente d'une greffe: d'un côté ceux qui sont (ou ont été) des donneurs d'organes potentiels et identifiés comme tels officiellement, de l'autre ceux qui ne sont pas donneurs d'organes (parce qu'ils sont opposés au don d'organes ou parce qu'ils n'ont jamais pris position sur le sujet). Le principe de réciprocité implique que le malade donneur potentiel est prioritaire sur le malade non donneur potentiel pour bénéficier d'un nouvel organe. Autrement dit, celui qui aurait accepté de donner «mérite» 
davantage de recevoir que celui qui n'aurait pas été prêt à faire le même geste envers autrui.

Si ce modèle suscite des réactions d'opposition forte, à l'instar de Julie (AR, comité) qui le qualifie de «discriminatoire », l'assentiment qu'il recueille de la part de plusieurs acteurs associatifs est essentiellement fondé sur l'idée de cohérence morale dans un contexte de ressources rares. «Ils sont contre [le don de leurs organes], mais le moment où ils doivent recevoir [...] un bout de machin, même une valve... puisque ça va avec la transplantation, ils sont pour, donc il y a quelque chose qui n'est pas décent »(Suzanne, AG, membre). Être prêt à donner est perçu comme une condition préalable pour bénéficier de la disposition d'un autre à donner. Léo, qui par ailleurs rejette fermement le principe du consentement présumé, distingue très clairement les deux catégories de malades décrites plus haut; «je ne le cache pas, c'est un petit peu radical de dire... quelqu'un qui a dit "non" pour la transplantation, il ne reçoit pas d'organe s'il en a besoin... on ne peut pas être receveur et pas donneur [en même temps]»(AG, membre). Celui qui prétend à un organe alors que lui-même refuse l'acte de donner qu'il accepte pourtant de la part d'autrui pour le sauver fait preuve d' «égoïsme pur ${ }^{567}$. » Les attitudes favorables au modèle de la réciprocité semblent ainsi prendre appui sur des motifs variables plus ou moins explicites, comme sanctionner les non-donneurs, inciter les indécis à se déterminer, ou encore témoigner une forme de reconnaissance à l'égard des donneurs potentiels.

Dans les raisonnements de ceux qui ne verraient pas d'un mauvais œil l'introduction du principe de réciprocité, le jugement moral (c'est-à-dire «qui mérite prioritairement de recevoir un organe?») tient une place bien plus importante que les effets que pourrait avoir une telle mesure sur le nombre de donneurs d'organes. Certains interviewés relèvent néanmoins les limites du modèle de la réciprocité, en premier lieu le risque de glissement d'une intention altruiste

567 Cela fait écho à la figure du "passager clandestin" (free rider) pour reprendre un concept classique en sciences sociales, faisant référence aux individus qui cherchent à profiter de l'action collective sans y participer. Voir notamment Olson M., Logic of Collective Action, Harvard University Press, 1965. 
vers une stratégie utilitariste. Le modèle de la réciprocité peut en effet se comprendre selon une logique assurantielle répondant d'abord à un intérêt personnel - la volonté d'être donneur pouvant découler de l'anticipation du besoin éventuel d'un organe, et non d'un désir authentique d'aider autrui. On retrouve ici, comme précédemment, la représentation et la préoccupation d'un don pur comme valeur cardinale de la régulation de la circulation des organes.

Les modèles alternatifs au consentement explicite - pensés généralement dans les milieux politiques, économiques ou éthiques comme autant de "solutions » potentielles au problème de la pénurie d'organes - font apparaître des positions contrastées parmi les acteurs associatifs ${ }^{568}$. Les divergences tiennent à l'importance variable qu'ils accordent à la question de l'augmentation du nombre de donneurs et aux enjeux éthiques qui eux-mêmes se définissent de façons différentes. En témoignent les tensions entre le respect de la volonté du donneur déclaré, l'intérêt du receveur potentiel, et le rôle des proches dans la décision de prélèvement. Dans l'ensemble, les propos des interviewés indiquent clairement que la fin ne justifie pas les moyens: le besoin d'organes ne peut légitimer n'importe quelle mesure ou solution. À cet égard, certains modèles de régulation suscitent de la méfiance lorsqu'ils paraissent enfreindre la nature fondamentale du don. La gratuité et le don volontaire (ou altruiste) s'apparentent à deux principes intangibles qui définissent l'authenticité du geste. Le souci de préserver le système du don d'organes de la sphère marchande et des raisonnements économiques montre que les considérations uniquement comptables dans la lutte contre la pénurie sont jugées irrecevables ${ }^{569}$. Cette «économie morale» du

568 Dans le cadre de la procédure de consultation de l'avant-projet de la loi fédérale sur la transplantation, les associations de patients qui se sont exprimées n'avaient pas de position commune quant au modèle de consentement préconisé (Chenaux 2000, 99).

569 La question de savoir si une incitation financière permet d'accroître le nombre de donneurs est hautement spéculative. Selon une étude américaine, elle entraînerait une augmentation des dons de reins inter vivos (Peters et al. 2016). Une étude autrichienne montre que des incitations financières dans le cadre du don cadavérique produisent des attitudes défavorables au don (Mayrhofer-Reinhartshuber et al. 2006). Ce résultat appuierait l'hypothèse de Titmuss (1970) selon laquelle, dans le cas du don de sang, l'introduction de dons rémunérés conduit à pervertir la fiabilité du système et à 
don d'organes observée parmi les acteurs associatifs coïncide en grande partie avec celle qui prévaut dans la population, que ce soit en Suisse ou dans d'autres pays ${ }^{570}$.

\subsection{Organisation hospitalière et procédures médicales}

Les critiques du manque d'engagement de l'État et de la LTx forment la trame principale de la perception du don d'organes comme problème politique. Le deuxième cadre d'interprétation qui émerge des entretiens concerne l'organisation du système de soins, en particulier les pratiques et attitudes des professionnels intervenant directement dans le processus du don d'organes. Le don d'organes apparaît ainsi également comme un problème hospitalier.

Pour bon nombre d'acteurs associatifs, l'enjeu de la pénurie d'organes se situe aussi à l'intérieur même des murs de l'hôpital, impliquant les équipes soignantes et l'organisation du travail, dans les services de soins intensifs notamment. L'hôpital est alors perçu comme un chaînon décisif de la circulation des organes. Cette dimension stratégique de l'hôpital par rapport au nombre d'organes disponibles a été mise en exergue par la moitié des interviewés. Pour quelques-uns, la question hospitalière est une priorité dans la lutte contre la pénurie, tandis que pour d'autres elle n'est qu'un facteur parmi d'autres. Si les pratiques hospitalières sont reliées au manque d'organes, c'est essentiellement en raison d'une détection non optimale des donneurs potentiels. En d'autres termes, l'argument de certains interviewés est que des organes sont "perdus» parce que l'identification des personnes en état de mort cérébrale (qui suppose un dispositif technique spécial, des compétences particulières et des examens spécifiques) n'est pas systématique, ou parce que la question du don d'organes n'est pas systématiquement abordée avec les proches. Deux causes principales sont avancées pour expliquer

diminuer le nombre de (bons) donneurs. Plus largement, si "l'efficacité du marché des organes reste à ce jour une hypothèse" (Sambuc 2012, 63), celle des autres modèles pour lutter contre la pénurie d'organes, y compris le consentement présumé, est également incertaine (Abadie et Gay 2006, Shepherd, O'Carroll et Ferguson 2014).

570 Bosisio, Santiago et Benaroyo 2011, Hoeyer, Schicktanz et Deleuran 2013. 
cette situation: l'insuffisance des moyens dans les hôpitaux d'une part, les attitudes et pratiques professionnelles d'autre part.

\section{Augmenter les moyens hospitaliers}

Une partie des interviewés pointent du doigt le manque de personnel spécialisé dans le don d'organes dans les hôpitaux régionaux et périphériques, qui compromet le repérage systématique et la prise en charge requise des donneurs en état de mort cérébrale. Pour Brigitte, qui affirme que «beaucoup d'organes [y] sont perdus ", des ressources supplémentaires devraient être allouées; «je trouve que [Swisstransplant] pourrait faire encore un travail plus en profondeur dans les petits [hôpitaux], là il y a un grand besoin!» (AR, comité). La dotation en personnel spécialisé dans les hôpitaux non transplanteurs est jugée encore insuffisante; "on a discuté souvent de ce problème... il y a beaucoup de petits hôpitaux où il n'y a pas un service qui est justement en place, pour détecter un donneur potentiel au moment où il y a quelque chose qui se passe " (Stéphanie, AR, comité). Cette dimension des ressources humaines et son influence sur les étapes médicales de la chaîne du don d'organes s'appuient souvent dans les entretiens sur les données d'une étude suisse récente qui a montré la pertinence de s'intéresser aux pratiques hospitalières ${ }^{571}$.

Le cas de l'Espagne est souvent cité en exemple pour souligner l'importance de l'organisation du système de soins et des pratiques professionnelles. Plusieurs interviewés louent la politique très active en faveur du don d'organes qui y a été mise en place; "les hommes politiques ont fait une campagne dans tous les hôpitaux: "Vous devez détecter vos donneurs" et [...] ils sont maintenant à quasiment 38 donneurs par million d'habitants... parce qu'ils ont formé des gens pour travailler dans une unité de soins intensifs, ils ont fait ce qu'il fallait pour que ces donneurs soient détectés, que la chaîne soit complète » (Murielle, FD, comité). Le taux spectaculaire de prélèvements en Espagne amène nombre d'interviewés à en faire un

571 Voir Weiss et al. 2014. La dernière partie de l'ouvrage montre que des mesures importantes ont été prises depuis pour améliorer les ressources pour le don d'organes dans les hôpitaux régionaux en Suisse. 
modèle dont la Suisse devrait s'inspirer pour lutter contre la pénurie d'organes; "une solution un peu comme l'Espagne... dans chaque région, il y a des coordinateurs [...] dans les hôpitaux, il y a des gens qui sont nommés pour faire avancer ce don d'organes... et là-bas, il y a plus de $30 \%$ de donneurs, ça marche! (Brigitte, AR, comité). La comparaison entre les deux pays suscite parfois des réactions d'indignation et d'incompréhension face au retard qu'accuse la Suisse; "moi ça me révolte que dans un pays comme la Suisse, on ne mette pas les moyens pour avoir des équipes formées, pour identifier les donneurs potentiels en dehors des hôpitaux universitaires » (Pascal, AR, membre). Certains acteurs associatifs soulignent que l'action publique au niveau hospitalier et du personnel soignant est plus déterminante que le mode de consentement pour améliorer la situation du don d'organes; "ça ne suffirait pas de mettre en place ce système de consentement présumé sans un très, très grand effort de formation et mise à disposition de moyens dans les hôpitaux périphériques, non universitaires pour qu'il y ait des équipes qui puissent identifier les donneurs potentiels parce qu'on en perd une quantité » (Pascal, AR, membre). Si quelques interviewés mentionnent également la nécessité de renforcer les compétences de communication des professionnels qui sont en lien avec les proches d'un donneur potentiel, le repérage non optimal des donneurs potentiels tient principalement à la dénonciation du manque de ressources personnelles dédiées spécifiquement aux différentes activités en lien avec le don d'organes au sein des établissements hospitaliers.

Malgré leurs critiques, la plupart des acteurs associatifs saluent aussi les améliorations significatives introduites depuis plusieurs années en milieu hospitalier, dans le cadre de la LTx ou plus souvent du Programme latin de don d'organes (PLDO). Plusieurs d'entre eux mettent en évidence le rôle positif du PLDO dans l'amélioration sensible de l'activité de prélèvement en Suisse romande et au Tessin. Sont notamment évoqués les moyens accrus en personnel spécialisé (postes de coordinatrices notamment) donnés ces dernières années à l'ensemble des hôpitaux disposant d'unités de soins intensifs afin d'améliorer le repérage des donneurs potentiels; «je pense que le fait de mettre un référent par hôpital, par région, et que cette personne 
s'engage, que ça soit dans son cahier des charges, ait la mission d'aller aux soins intensifs tous les cas où il y a un risque de décès, d'aller voir la famille pour préparer l'éventualité, je pense que ça c'est très très positif » (Léo, AG, membre).

\section{Réticences professionnelles}

Le non-repérage systématique des donneurs potentiels en état de mort cérébrale est aussi relié dans notre corpus à un manque d'implication de certains professionnels. Bien que cette dimension individuelle ne soit pas totalement indépendante, comme on va le voir, des enjeux politiques et légaux de la régulation du don d'organes, certains acteurs associatifs mettent l'accent sur un problème de personne, au-delà d'un problème de personnel. Faisant référence aux variations importantes du taux de donneurs observées il y a quelques années entre les différentes parties de la Suisse, Léo souligne que la pénurie est aussi «une question de volonté médicale, [...] une question d'éthique ou de conception de l'état d'esprit» (AG, membre).

Plusieurs interviewés rappellent que si le don d'organes reste un sujet sensible qui ne fait pas forcément l'unanimité dans le corps social, il peut donner lieu à des hésitations au sein même du monde hospitalier. Invoquant souvent à cet égard le contexte lourd et dramatique des situations concernées, ils comprennent, avec une certaine indulgence, la difficulté que peuvent rencontrer les équipes soignantes lorsqu'il s'agit d'annoncer la mort d'un proche et d'aborder la question du don d'organes; "c'est toujours des drames, forcément, ce n'est pas toujours facile face à une famille, face à une jeune femme qui a trois enfants et dont le mari vient de décéder d'aller encore demander des organes... psychologiquement c'est peut-être un peu plus... alors certains le font...» (Léo, AG, membre).

Plus souvent, les acteurs associatifs suggèrent que certains professionnels feraient preuve à certaines occasions d'une désinvolture coupable en ne mettant pas tout en œuvre pour identifier les donneurs potentiels. L'investissement pour le don d'organes ne serait ainsi pas toujours à la hauteur des exigences. Pour Bernard par 
exemple, le degré de conviction personnelle joue un rôle déterminant dans les chances d'obtenir des organes; "si c'est un [professionnel] qui fait parce qu'on lui a dit de faire, ça ne va pas jouer [...] il faut déjà convaincre ces gens-là de l'importance du don d'organes [...] et là il y a beaucoup à faire" ( $\mathrm{AG}$, comité). Jean dénonce quant à lui les réticences qui peuvent exister par rapport à la médecine de transplantation; "un des problèmes, c'est qu'il y a certains médecins chirurgiens qui ne sont pas favorables à la transplantation... qui disent: "Oui, mais cela coûte cher et puis c'est la sélection naturelle" " (AR, comité). D'autres acteurs associatifs insistent sur le fait que la difficulté de l'approche d'une famille endeuillée ne saurait en aucun cas justifier de renoncer à la démarche auprès des proches. Évoquant la surcharge de travail qu'un donneur potentiel implique aux soins intensifs, Adeline souligne l'impératif d'un engagement professionnel sans faille pour le don d'organes; "on ne peut pas prendre un organe comme ça [...] les médecins théoriquement ont, on m'a dit, l'obligation de faire tous les tests, seulement s'il y a un médecin qui a rendez-vous à 8 heures avec sa petite amie, et que c'est le premier rendez-vous, eh bien il ne va pas sacrifier cet amour promis pour un mort! Et ça, c'est reconnu, enfin c'est très schématique, mais ils ont leurs heures aussi les médecins " (AG, comité). Pour expliquer que certains médecins ne seraient pas exemplaires en matière d'identification des donneurs potentiels, tout en relevant une amélioration au cours des dernières années, quelques interviewés évoquent le moindre prestige social et professionnel des activités de diagnostic de la mort cérébrale et de prélèvement d'organes; «implanter l'organe, c'est prestigieux, par contre, prélever les organes, c'est pratiquement du bénévolat...»(Charles, AG, membre).

Évoquant les « réticences » de certains médecins, Marc dénonce les effets négatifs d'une forme de libéralisme dans l'éthique professionnelle des différents acteurs hospitaliers du don d'organes; "le respect des convictions de chacun, ça commence vite à poser problème $[. .$.$] quand on est dans une microsociété comme les urgences$ ou les soins intensifs [...] il suffit qu'il y ait deux personnes qui soient farouchement opposées au don, et tout d'un coup on ne peut pas 
le faire parce que les freins vont s'exprimer de manière tout à fait forte » (FD, comité). Il fustige ainsi l'absence d'obligation réelle pour les professionnels en matière de repérage des donneurs en état de mort cérébrale. Trop libérale, la loi agirait au détriment de l'intérêt des malades en attente d'une greffe. Par son laxisme à l'égard des attitudes timorées ou des "conflits de conscience" de certains professionnels vis-à-vis du don d'organes, l'organisation hospitalière et le cadre législatif plus largement sont, aux yeux de Marc, en partie responsables de la pénurie et de patients morts faute d'avoir été greffés à temps; " [il faut] obliger les centres médicaux avec des soins intensifs à détecter de manière systématique les personnes qui soient des potentiels donneurs... ça me semble être la base!»

Le point de vue de Marc est singulier: il est quasiment le seul dans notre corpus à dénoncer si vigoureusement l'attitude de certains médecins et à lier étroitement le travail des soignants et le rôle du cadre législatif. Néanmoins, il fait figure de révélateur grossissant d'une dimension importante de la perception du problème de la pénurie d'organes: l'opinion personnelle vis-à-vis de la transplantation des professionnels impliqués dans les procédures du don. La vigueur des critiques de Marc, et de quelques autres, à l'égard d'une partie du monde hospitalier ne doit probablement pas être prise au pied de la lettre, mais interprétée à l'aune de la sensibilité de cet enjeu pour les acteurs associatifs, engagés bénévolement en faveur du don d'organes. La dénonciation reflète l'indignation vis-à-vis de l'idée qu'il puisse y avoir des résistances au sein même du corps médical et qu'elles contribuent à la pénurie de greffons - alors que structurellement patients et médecins sont alliés dans la défense de la même cause. L'indignation peut tenir aussi à l'idée que la chance d'une greffe dépende d'un individu, de ses convictions ou de son degré d'engagement professionnel. L'existence de dysfonctionnements au cœur du dispositif du don d'organes peut alors apparaître comme particulièrement choquante, d'autant que les groupes de patients ont peu prise sur cet aspect. Rien ne permet cependant de se prononcer sur l'ampleur de ces résistances et leur impact sur l'activité de transplantation - les situations évoquées dans les entretiens 
sont certainement très marginales dans les faits ${ }^{572}$. Pour les acteurs associatifs, les réticences médicales sont moins tolérables et compréhensibles que celles de la population générale. Cela montre que le monde hospitalier, de par sa position cruciale dans la chaîne du don d'organes, reçoit une obligation d'exemplarité et d'excellence, sur les plans technique, organisationnel, et de la conscience professionnelle.

\subsection{Conclusion}

Dans ce chapitre, nous avons analysé deux cadres majeurs de la perception du don d'organes par les acteurs associatifs. Le premier renvoie à une lecture du don d'organes en tant que problème politique et législatif qui repose essentiellement sur la pénurie persistante de greffons et sur le contenu de la LTx. Empreinte de désenchantement, d'incompréhension ou de colère, cette lecture politique dénonce le manque de soutien des autorités fédérales à la cause du don d'organes. Le ressentiment exprimé par certains interviewés à l'égard du monde politique doit être replacé dans le contexte particulier de l'élaboration de la LTx dont la teneur (le consentement explicite et la neutralité de l'État sur la question) est loin d'avoir répondu aux attentes des milieux intéressés, espérant des mesures concrètes et un positionnement officiel clairement en faveur du don d'organes. La critique du consentement explicite des acteurs associatifs ne s'inscrit pas dans une vision strictement instrumentale de l'action publique (augmenter l'offre de greffons) et renvoie à l'importance symbolique attribuée à la loi. À leurs yeux, la LTx incarne l'échec d'une véritable reconnaissance par l'État du don d'organes comme problème public. Si les différents modèles de circulation des organes révèlent un consensus autour de la préservation du don hors de la sphère marchande, l'idée du don authentique ou l'esprit du don n'a pas le même sens pour tous

572 Une étude suisse avait par exemple montré, il y a une vingtaine d'années, que le don et la transplantation d'organes suscitaient des interrogations, notamment quant à la mort cérébrale, parmi les médecins et le personnel infirmier (Imperatori et al. 2001). Selon une étude suédoise, les médecins travaillant dans les soins intensifs approchent les patients avec des attitudes différentes à l'égard du don d'organes: en faveur du don, neutre ou ambivalente (Sanner 2007). 
les acteurs associatifs, renvoyant à des valeurs différentes et à des équilibres variables entre les trois pôles de l'espace du don que sont l'intérêt individuel, l'intérêt familial et l'intérêt collectif ${ }^{573}$. Le deuxième cadre de perception analysé dans ce chapitre présente le don d'organes comme un problème hospitalier. Deux aspects ont été plus particulièrement mis en avant par les acteurs associatifs: d'une part la question des ressources financières et en personnel spécialisé dans les hôpitaux, d'autre part la question des convictions individuelles du personnel impliqué directement dans les procédures menant à un don d'organes. Ces deux «causes» de la situation problématique du don d'organes en Suisse font très souvent écho, en filigrane, à un troisième cadre majeur d'interprétation: celui de l'information, objet du chapitre suivant qui aborde la représentation de la disposition à donner de l'individu lambda et la nature de l'information que les citoyens devraient recevoir.

573 Steiner 2010. 


\section{Chapitre 8}

\section{Les enjeux de l'information et la perception du public}

\section{Raphaël Hammer}

L'information de la population constitue le troisième enjeu majeur de la perception du don d'organes comme problème public. La plupart des acteurs associatifs voient dans la quantité et la qualité de l'information transmise à la population à la fois l'une des causes principales de la pénurie d'organes en Suisse, en même temps qu'une partie de la solution. Au-delà des critiques adressées aux campagnes de l'OFSP, les interviewés dénoncent de manière plus générale l'«information insuffisante» des citoyens, la retenue et la discrétion des messages publics - autant de signes de l'engagement timoré de l'État pour le don d'organes. De fait, l'importance du thème de l'information constitue la population dans son ensemble comme un public politique du don d'organes.

Ce chapitre aborde dans un premier temps de quelle manière est perçue la population en tant que groupe de donneurs potentiels. Si les interviewés partagent une vision «optimiste» de la réceptivité des citoyens à la cause du don d'organes, plusieurs facteurs sont avancés pour expliquer néanmoins le climat d'indifférence et de réticences qui caractérise une partie de la population. Dans les discours des acteurs associatifs (et d'autres acteurs du don d'organes), le «manque d'informations» se présente souvent comme une antienne relativement creuse, sans que l'on sache ce que serait concrètement 
une "meilleure information " ou "plus d'information ", ne serait-ce qu'en termes de contenu ou de finalité. L'analyse permet néanmoins d'identifier plusieurs fonctions que les interviewés associent à une véritable politique de l'information. Nous décrirons ainsi dans un second temps ces différentes significations, en montrant que les mesures imaginées par les acteurs associatifs pour améliorer la situation du don d'organes révèlent parfois des objectifs divergents de l'action publique.

\subsection{Une population a priori acquise au don d'organes}

La question de l'action publique en matière d'information est d'autant plus saillante dans les discours des acteurs associatifs qu'ils partagent une vision plutôt optimiste de la disposition des individus à faire don de leurs organes.

S'appuyant sur leurs expériences concrètes au contact de la population ou sur les résultats de sondages, nombre d'interviewés sont convaincus que la grande majorité des gens sont a priori bien disposés par rapport à la question du don d'organes; "des audits ont été faits en Suisse, le peuple suisse n'est pas contre le don d'organe bien évidemment, ni contre la transplantation» (Murielle, FD, comité). Selon Marc qui estime qu'environ sept citoyens sur dix sont favorables au don d'organes, "on devrait avoir en Suisse romande 100, 120 donneurs par année, et puis on en a 30,40, donc on en a trois fois moins de ce qui serait envisageable, donc c'est là où je me dis "oui, on a fait des choses", mais aujourd'hui, je me rends compte que la population est acquise et que l'enjeu se situe ailleurs» (FD, comité). Pour la plupart des interviewés, nombre de citoyens sont susceptibles, à peu de frais, de se transformer en donneurs déclarés et convaincus; " je pense que beaucoup de leurs réticences s'annulent lorsqu'on discute un peu avec eux, voilà... d'où l'information et la discussion» (Pascal, AR, membre).

Ce décalage est attribué au fait que les individus ne sont pas incités à faire l'effort de se pencher véritablement sur la question du don d'organes, ou à matérialiser leur décision en signant une carte de donneur ou en informant l'entourage de leur choix; "le 
problème c'est que quasiment le $80 \%$ ou le $90 \%$ des gens [avec qui j'ai parlé] sont d'accord, mais ils n'ont pas préparé le moment où éventuellement ils auraient un accident et puis ils seraient donneurs, par rapport à leur famille» (Laurent, FD, membre). L'interprétation du manque de donneurs potentiels en termes de dispositions négatives des individus est marginale dans notre corpus. Les acteurs associatifs qui invoquent «l'individualisme forcené » (Richard, AR, membre), le "chacun pour soi » (Bernard, AG, comité), le manque de solidarité ou d'" empathie» (Christophe, FD, membre) sont des exceptions. Dans leur grande majorité, les citoyens ne sont donc pas perçus comme foncièrement opposés au don d'organes. La pénurie n'est pas considérée comme le fait d'un public qui serait globalement défavorable ou hostile à l'égard de la transplantation; «il y a des gens qui sont contre, mais quand on discute, quand on a des stands, la plupart des gens, ils sont quand même pour le don d'organes " (Julie, AR, comité). La position de Sébastien résume l'attitude générale de notre corpus: "Je pense que les gens sont bons et généreux; seulement il faut juste bien leur parler, il faut leur parler juste» (AG, membre). La pénurie d'organes est ainsi moins considérée comme le reflet des attitudes de la population mais comme le révélateur d'une politique d'information déficiente.

\subsection{Les causes de l'indifférence et des réticences du public}

Comment les acteurs associatifs rendent-ils compte de ce problème de concrétisation de la décision d'être donneur et des hésitations, réserves ou appréhensions vis-à-vis du don d'organes qu'ils perçoivent dans une partie de la population? Rejetant les explications en termes d'égoïsme ou d'opposition de principe au don, les interviewés attribuent l'inertie ou le manque de mobilisation concrète de la population à deux facteurs principaux: l'implication personnelle faible du citoyen ordinaire d'une part, les peurs et fantasmes autour du prélèvement d'autre part, qui bien souvent sont perçus en lien étroit avec des connaissances factuelles lacunaires sur le don d'organes. 


\section{Un sujet éloigné des préoccupations quotidiennes}

Si les individus ne sont pas enclins à réfléchir sérieusement à la question du don d'organes, c'est parce qu'ils ne sont pas ou ne se sentent pas concernés. C'est là une première raison majeure évoquée par les interviewés: ne pas être touché personnellement constitue un facteur d'ignorance et de désintérêt vis-à-vis du problème. À leurs yeux, le don d'organes est un sujet légitime de préoccupation pour la majorité de la population, mais qui est ressenti comme "lointain» et «distant ", comme un problème parmi d'autres; "tant que l'on n'y est pas confronté, ou que l'on n'a pas dans son entourage une personne vraiment confrontée à la problématique d'avoir besoin d'une transplantation, c'est un sujet un peu comme un autre " (Didier, $\mathrm{AR}$, comité). De fait, les individus ne sont pas incités à se poser la question du don; «il y en a beaucoup qui n'y pensent même pas, moi je pense qu'il y a une grosse partie des gens qui n'y pensent pas» (Laura, AR, comité). Pour les acteurs associatifs, tant que les gens ne sont pas affectés à titre privé, ils restent hermétiques aux messages habituels sur le don d'organes («sauver une vie» ou «une fois décédé, nos organes peuvent servir à quelqu'un d'autre ») qui ont alors une tonalité très abstraite.

À l'inverse, être soi-même greffé, connaître dans son entourage une personne transplantée ou en attente de greffe, ou encore quelqu'un qui a fait don d'un de ses organes, conduisent naturellement à se sentir touché et à prendre position. Pour Léo, la motivation au don est directement liée à un élément de vécu personnel, à un déclencheur émotionnel; "je pense qu'il y a quand même pas mal de gens qui deviennent donneurs quand ils ont eu dans la famille ou dans leurs proches, des connaissances, quelqu'un qui a reçu un don d'organes» (AG, membre). Se sentir «naturellement» concerné par le don d'organes dépend donc des circonstances individuelles, des situations particulières, des expériences personnelles - autant d'éléments contingents qui échappent à l'action publique. Comme nous le montrons plus loin, les acteurs associatifs sont persuadés qu'il est malgré tout possible de "toucher", de sensibiliser les individus, même s'ils ne sont pas directement concernés. Et c'est bien parce que 
l'engagement concret de l'homme de la rue pour le don ne va pas de soi que les interviewés considèrent que l'information du public doit servir de stimulus à la réflexion et aider à la concrétisation des bonnes intentions latentes des citoyens.

\section{Peurs, tabous et méconnaissances}

Les idées préconçues et une part d'ignorance sont également des facteurs invoqués pour expliquer l'inertie d'une partie du public vis-à-vis du don d'organes, les réticences à en parler et à se poser la question. Par exemple, Daniel observe que "même si le don d'organes est en évolution, il y a encore beaucoup de tabous, des résistances chez des gens qui a priori ne seraient pas forcément opposés » (AG, comité). La méfiance et la peur du public sont alimentées par la méconnaissance ou l'ignorance du public; «il y a quand même beaucoup de gens qui connaissent pas comment cela se passe, et quand on ne connaît pas quelque chose, on a peur, donc ça, c'est peut-être pourquoi on dit non » (Nadia, FD, comité).

Au registre des croyances, il est notamment fait allusion au fantasme selon lequel les donneurs potentiels seraient moins bien pris en charge aux soins intensifs, voire ne seraient pas soignés du tout; "des réactions qui sont totalement ridicules je pense, mais primaires [...] il y a des gens qui croient que l'on va les laisser mourir pour que l'on puisse prendre leurs organes " (Claude, AG, comité). Pour quelques interviewés, la figure du médecin transplanteur comme vautour, à l'affût de cadavres à prélever, peut expliquer chez certaines personnes la crainte d'être prélevées sans être véritablement mortes. D'autres font référence à la croyance que le cadavre du donneur n'est pas respecté; "quand on dit: "Mais non, je suis mort, oui, mais je ne veux pas qu'on me charcute, je tiens...” [...] ce n'est pas vrai du tout, parce qu'ils [l'équipe de prélèvement] ont quand même un grand respect, d'autant plus qu'ils [les donneurs] donnent leurs organes, d'autant plus qu'on doit avoir du respect pour eux, mais il y en a qui ne sont pas encore convaincus encore de ça " (Thomas, $\mathrm{AR}$, comité). Dans un autre registre, quelques interviewés évoquent des informations erronées, comme croire qu'il y a une limite d'âge 
prédéfinie pour être donneur, ou que seules les personnes en excellente santé peuvent être prélevées.

Aux yeux de nombreux acteurs associatifs, la question de la mort, indissociable du thème du don d'organes, constitue un tabou qui pèse lourd. La peur de la mort expliquerait une partie des résistances. Pour Claude, comme pour d'autres interviewés, le sujet du don d'organes suscite encore bien souvent une gêne, un malaise; «il y a une crainte parce que cela fait peur à beaucoup de gens le don d'organes [...] la mort est là, donc il y a ce paradoxe de la mort qui donnera la vie, ou qui aidera à la continuer ou à l'améliorer, et ça c'est pas facile à résoudre » (AG, comité). La plupart des acteurs associatifs indiquent comprendre ce tabou de la mort et la réticence à penser sa mort prochaine, qui en soi ne sont pas perçus comme des attitudes contre le don d'organes; "c'est vrai que discuter du don d'organe, souvent c'est discuter de la mort, et parler de la mort, c'est sensible [...] je comprends leurs doutes, leurs craintes, leurs peurs, et je les respecte» (Pascal, AR, membre). Pour Sylvie, c'est précisément le «confort» existentiel dans lequel les gens vivent qui participe d'une évacuation de la réflexion sur la mort et sa propre mort; «moi je pense qu'on oublie qu'on va mourir, moi je crois que c'est ça le problème» (AG, membre). Aux yeux de quelques interviewés, ce contexte psychologique et culturel difficile autour de la mort dans nos sociétés ${ }^{574}$ explique aussi en partie l'engagement timoré du politique vis-à-vis du don d'organes; "c'est quand même un truc qui est un peu tabou, on parle de la mort [...] je pense que c'est aussi pour cela que politiquement ça a un peu de la peine à bouger parce qu'aucun politique ose prendre la décision» (Laurent, FD, membre).

\section{La mort cérébrale}

Entre peur et méconnaissance, la question de la mort cérébrale émerge comme une difficulté supplémentaire. Sans nécessairement établir un lien direct entre la problématique de la mort cérébrale et la réflexion individuelle sur le don, les interviewés mettent en avant la représentation floue du public de cette définition de la mort

574 Boileau 2002. 
éloignée des conceptions traditionnelles ${ }^{575}$. À cet égard, Stéphanie résume les interrogations cognitives que peut avoir la population, ainsi que les préoccupations éthiques et scientifiques autour de la certitude du décès: "quand on parle de mort cérébrale, il y a aussi ce travail à faire par rapport au coma et à la mort cérébrale, ils confondent, c'est un peu: “oui, on n'est pas mort et vous voulez nous prendre des organes", ça c'est la question, "est-ce qu'il n'y a pas d'erreurs?", “est-ce que vous êtes sûr que l'on est mort?", donc il faut les rassurer, on doit leur expliquer comment cela se déroule, qu'ils font des tests, on essaie d'expliquer finalement tout ce que l'on peut expliquer à une personne qui ne connaît rien [...] voilà on est là pour répondre à toutes les questions que les gens se posent, et surtout pour essayer d'expliquer en fait que ce n'est rien de dramatique, que l'on est mort, et de les persuader de prendre la carte» (AR, comité).

Plusieurs acteurs associatifs insistent sur la nécessité d'une démarche d'information objective des enjeux du don d'organes comme préalable à des messages de sensibilisation. Les propos de l'un d'eux illustrent cet impératif pédagogique, sans lequel la communication publique risque d'être vaine, faute d'avoir posé les données factuelles dont les citoyens ont besoin avant d'engager une réflexion sur la question du don. Il est ici fait référence à la campagne de l'OFSP menée en 2008 dont l'une des affiches mettait en avant le principe d'autodétermination avec le slogan "C'est moi qui décide ${ }^{576}$ "; " “C'est moi qui décide quoi!?” Il faut parler du don d'organes, les gens ne savent pas; il faut refaire tout le chemin, expliquer la mort cérébrale... ce n'est pas évident, parce qu'il n'y a pas longtemps encore, la mort c'était le cœur qui s'arrête, pour tout le monde, la mort cérébrale il faut l'ingurgiter, ce n'est pas évident, il faut expliquer ce que c'est, il faut expliquer ce que c'est qu'une transplantation, etc. Il ne faut pas montrer seulement des gens heureux comme moi d'être en santé, ça ne suffit pas » (Patrick, AG, membre).

575 Idem.

576 Les deux autres slogans des affiches de la campagne 2008 de l'OFSP indiquaient: "Je sais ce que je veux", et "Qui d'autre sait ce que tu veux?" Les trois slogans étaient accompagnés de la mention "Don d'organes. Oui ou non?" en caractères plus petits. 
La mort cérébrale suscite des appréhensions mais qui peuvent être surmontées par un dialogue fondé sur des informations précises et accessibles; "cela fait peur à tout le monde... mais une fois qu'ils comprennent ce qu'est la mort cérébrale... voilà il faut toujours leur expliquer ce que cela veut dire" (Brigitte, AR, comité). La notion de mort cérébrale s'ajoute ainsi à d'autres éléments de crainte et d'incompréhension qui ne facilitent pas une appréhension sereine du don d'organes, et rendent malaisé le dialogue avec la population. Certains interviewés soulignent que cette notion est pour eux-mêmes une difficulté objective lorsqu'il s'agit de donner une explication intelligible et convaincante à l'homme de la rue; "c'est le plus difficile à expliquer lors de la journée du don d'organes, la mort cérébrale, très compliqué, “on est vraiment mort?”, ça c'est les questions des gens» (Pierre, AR, comité).

\section{Réticences et oppositions du public}

Les réticences à l'égard du don d'organes sont souvent envisagées comme pouvant être surmontées ou atténuées par la transmission d'informations scientifiques et objectives sur les pratiques de la transplantation. Cependant, deux motifs d'opposition dans la population tendent à échapper, selon les acteurs associatifs, à l'influence possible d'une meilleure communication. Le premier concerne la volonté de préserver l'intégrité du corps du défunt. Le second tient aux croyances religieuses ou culturelles, qui peuvent être liées à la question du corps; "quelqu'un qui est très religieux... on peut imaginer qu'effectivement que ce n'est pas dans ses mœurs qu'on répartisse ses organes à quelqu'un d'autre» (Laurent, FD, membre); "il y a des puristes... il y a des catholiques fervents, oui, il y a aussi peut-être une question de religion» (Daniel, AG, comité). L'analyse de ces deux thèmes revient plus largement à s'interroger sur la manière dont les attitudes de refus du don d'organes sont interprétées, voire légitimées.

Certains acteurs associatifs affichent une attitude libérale et compréhensive, quand bien même l'opposition ne serait pas motivée par une conviction forte. C'est le cas de Patrick: "Je pense que 
si quelqu'un ne veut pas avoir une carte, c'est sa liberté... pour quelle raison ?... Eh bien, il y avait cette amie qui m'avait dit: "J'ai l'impression de me déclarer morte" ou je ne sais pas quoi, ça peut être d'autres choses»(AG, membre). De même Léo se refuse à porter un jugement sur la recevabilité ou non des attitudes de refus et d'opposition, partant du principe qu'il s'agit d'un choix qui doit rester libre; "ça c'est chacun son truc [...] oui, il y a des raisons acceptables, toute raison est acceptable, j'entends, moi j'ai une amie, elle m'a dit: "Jamais je donnerai mes organes", un de nos meilleurs amis: "Je ne donnerai pas mes organes, moi je veux donner mon corps à la science" [...] elle a une idée, ce n'est pas à moi de juger si c'est une bonne raison ou pas, c'est sa raison" (AG, membre). Plusieurs interviewés mettent ainsi en avant le principe d'autodétermination, que l'on soit pour ou contre le don d'organes; "chacun a le droit d'avoir son idée [...] on a le droit de refuser, ce n'est pas un crime [...] il ne faut pas non plus imposer le don à tout le monde, s'ils n'en veulent pas, il n'y a pas de raison» (Marc, FD, comité). L'opposition au don d'organes ne constitue alors pas une opinion condamnable et s'avère tout à fait respectable dans la mesure où une réflexion a été menée sur le sujet. Rares sont ceux qui adoptent cette logique de valorisation de l'autodétermination, estimant qu'une plus grande visibilité des attitudes divergentes permettrait de nourrir le débat public; "on devrait en parler plus parce que chacun a la liberté de choix de faire ce qu'il veut [...] pourquoi il n'aurait pas le droit de dire non? C'est son choix, laissez-le dire son choix. Il a une couleur politique, il a une couleur de religion, mais c'est parfait! ça nous sert d'avoir des gens qui nous disent: "Je ne suis pas pour le don d'organes" (silence)... parce que ça veut dire qu'il y a déjà réfléchi et pensé »(Carine, FD, comité).

Cette posture libérale est le fait d'une minorité d'acteurs associatifs. Les oppositions franches au don d'organes ont plutôt tendance à susciter des réactions contrastées dans notre corpus, où le principe du respect du choix de chacun et l'incompréhension des motifs de refus s'entremêlent. Cette ambivalence peut être exprimée de manière subtile ou modérée, comme en témoigne l'extrait suivant: «Je conçois que les gens disent “non” parce qu'ils n'ont pas envie 
qu'on touche leur intégrité physique; ils sont nés comme ça, ils vont partir avec tout, même si on naît poussière et on repart poussière de toute façon... ouais, j'accepte parce que bon ben il y en a qui votent droite, $\mathrm{y}$ en a qui votent gauche, $\mathrm{y}$ en a qui donnent, $\mathrm{y}$ en a qui donnent pas, c'est un état de fait. Je crois que cela ne sert à rien de se battre contre des moulins à vent » (Murielle, FD, comité). De même, Georges indique respecter l'idée que le corps n'est pas un stock de pièces détachées, tout en l'estimant peu fondée: "Moi je suis, disons, assez libéral. C'est son avis, mais j'espère bien que cette personne-là n'aura pas besoin d'un don d'organes [...] parce que ce jour-là, on pourrait lui dire: "Écoutez, madame, malgré ce que vous dites, vous seriez bien contente d'en avoir un pour vous faire vivre plus longtemps" [...] c'est ce que je lui dirais» (AR, comité). Certains opposent aux refus fondés sur les convictions religieuses l'argument de l'éventualité du besoin personnel; «on n'insiste pas... je leur dis: "Eh bien, écoutez, moi aussi j’ai la foi, je crois en Dieu, mais cela peut arriver quand même que vous deviez passer par une transplantation, réfléchissez-y" [...] c'est leur vie, c'est le quotidien, c'est leur foi... nous on accepte qu'ils refusent de donner, il faut que cela mûrisse dans leur tête...» (Brigitte, AR, comité).

Le souci de l'intégrité physique du cadavre est aussi source d'étonnement; "[des gens] qui veulent être enterrés entier [...] leur corps doit être intègre quand ils sont [décédés]...» - attitude rencontrée "plus souvent que je ne le pensais oui [...] c'est assez surprenant » (Laurent, FD, membre). Ce motif d'opposition au don d'organes suscite également des réactions incrédules, voire critiques, à l'instar de Didier; «l'organe en soi, c'est du tissu, qui a une fonction, mais ça n'a pas d'âme $[. .$.$] c'est du tissu cellulaire point barre » (AR, comité).$ Compte tenu de son parcours, Didier revendique une conception mécanique et rationnelle du corps; "j'étais arrivé à un endroit où il y avait une pièce qui lâchait, j'avais besoin d'une pièce de rechange comme je disais avec l'exemple de la voiture, pour que je puisse continuer après, que je puisse continuer d'amener de l'argent, que je puisse continuer de construire quelque chose avec ma famille, bon je sais que peut-être c'est mon côté scientifique, un côté un peu 
cartésien, après il y a des gens qui penseront tout autrement ${ }^{577} \ldots$ » (AR, comité).

Si plusieurs acteurs associatifs expriment cette tension entre la norme du respect de la position de tout un chacun et l'incompréhension vis-à-vis de la volonté de préserver le corps intact, d'autres, minoritaires, ne sont guère ambivalents et affichent des réactions critiques. C'est le cas notamment de Christophe qui fustige les attitudes contre le don, les mettant sur le compte de croyances erronées; "une personne qui refuse d'être donneur, je trouve que cela est ridicule, il n'y a aucune raison de ne pas donner ses organes, on ne va pas utiliser ses organes pour jouer avec ». Et de souligner la vacuité de ce type de refus à travers l'évidence morale du geste conjuguée à celle du bénéfice médical pour les receveurs; "c'est simplement que cela sauve d'autres personnes [...] il y en a à qui il faudrait une cornée c'est tout, et grâce à cela les gens pourront voir un lever et un coucher de soleil, et voir des choses importantes, leurs parents, et voilà c'est de nouveau voir, il y a des gens qui pourront à nouveau respirer [...] vivre normalement, c'est merveilleux de pouvoir donner ses organes, même moi je suis donneur, et si je meurs et que mon cerveau s'éteint j'espère que mes organes pourront aller à d'autres personnes aussi » (Christophe, FD, membre). Aux yeux de certains interviewés, il ne peut véritablement y avoir de motifs de refus valables, ou du moins qui seraient à la même hauteur morale que l'enjeu de vie du don d'organes. Donner et pouvoir sauver une vie apparaissent ainsi dans certains discours comme un impératif moral qu'aucune raison «contre» ne peut disputer. La volonté de préserver l'intégrité du corps peut alors difficilement être qualifiée de «bonne » raison d'opposition au don et reflète une « réaction simpliste, très simpliste [...] si on peut donner un organe et sauver quelqu'un il faut le faire... chez les gens qui refusent, est-ce que c'est un égoïsme conscient? Je ne sais pas" (Claude, AG, comité). Dans cette logique, quelques interviewés indiquent clairement que

577 Plusieurs études suggèrent l'existence d'un lien entre la représentation profane de son corps (ou du corps en général) et le souci de l'intégrité du corps, et que ces aspects influencent l'attitude à l'égard du don d'organes (Boileau 2002, Sanner 2001, FalomirPichastor, Berent et Pereira 2013). 
l'opposition au don n'est pas une attitude fondée en valeurs, mais une réaction émotionnelle; "Non, non, je crois que... ce n'est pas des bonnes raisons, ce sont des raisons sentimentales, appeler cela des bonnes raisons, des mauvaises raisons, je pense que ce sont des mauvaises raisons [...] c'est avant tout l'émotionnel qui prend le dessus et ça c'est faux, mais ce sont des raisons »(Adeline, AG, comité).

Dans l'éventail des réticences plus ou moins fortes de la population vis-à-vis du don d'organes, l'intégrité du corps et les convictions religieuses occupent une place à part dans la mesure où elles sont volontiers associées à des croyances culturelles ou à des valeurs personnelles, voire à des mécanismes émotionnels. À quelques exceptions près, ces oppositions ne sont pas interprétées en termes d'ignorance, d'informations erronées, de peur ou de fantasme, et ne sont donc guère perçues comme étant sensibles à l'influence des actions publiques de communication ou de la discussion interpersonnelle. Au-delà de ces motifs de refus, les acteurs associatifs soutiennent que le manque de sensibilité du public n'est en rien rédhibitoire. Ils jugent que l'amélioration de l'information sur le don et la transplantation d'organes peut modifier positivement cette inertie. Nombre d'idées fausses seraient ainsi rectifiées par la transmission d'explications claires et objectives, par exemple sur la notion de mort cérébrale, sur la procédure diagnostique du décès ou encore sur la manière dont le corps est rendu à la famille. En d'autres termes, les acteurs associatifs se rallient assez largement à un modèle pédagogique de l'information du public dans la mesure où la diffusion d'une représentation du don et de la transplantation conforme aux pratiques en vigueur et aux faits scientifiques devrait permettre de rassurer, de surmonter certaines résistances ou craintes, et de convaincre.

\subsection{Sensibiliser le public}

L'importance que les acteurs associatifs accordent à l'information du public ne se résume toutefois pas à sa fonction pédagogique ou éducative. Nous avons relevé précédemment que ces derniers 
mettaient l'accent sur la difficulté centrale d'intéresser des individus à un sujet pour lequel ils ne se sentent pas concernés. Cette difficulté est au cœur de toute action politique ou humanitaire qui cherche à attirer l'attention d'un public qui a priori n'a pas de raison majeure d'y être réceptif ${ }^{578}$. L'enjeu est de sensibiliser, c'est-à-dire amener un ensemble d'individus, relativement indifférents vis-à-vis d'une question, à se sentir concernés personnellement, voire à modifier leur comportement ${ }^{579}$. Cette situation peut être rapprochée de la question $\mathrm{du}$ «concernement » analysée par Boltanski ${ }^{580}$ en lien avec les manières de décrire la souffrance d'individus afin de susciter la pitié du spectateur distant et l'amener à un engagement. La topique du sentiment, fondée sur l'émotion comme opérateur de rapprochement entre le public et les souffrants ${ }^{581}$, s'avère ici d'actualité, tout en n'épuisant pas la diversité des mesures préconisées par les acteurs associatifs lorsqu'ils en appellent à davantage d'information ou à une meilleure information. L'objectif de sensibilisation du public prend en effet des formes différentes, parfois opposées.

\section{Normaliser le don d'organes comme enjeu social}

Une première fonction attribuée à l'information du public consiste à normaliser le don d'organes comme enjeu de société. Aux yeux d'une majorité d'interviewés, le but est de faire entrer dans les mœurs le thème de la transplantation et d'intégrer la réflexion sur le don d'organes dans la culture contemporaine. Il s'agit de faire du don de ses organes un sujet ordinaire, ancré dans les mentalités. Regrettant que le don d'organes ne soit pas suffisamment publicisé, nombre d'acteurs associatifs plaident pour que l'État intensifie les campagnes d'information et multiplie les événements afin d'accroître

578 Cardon et al. 1999.

579 Traïni et Siméant définissent ainsi l'objectif de sensibilisation: "D'une part, rendre sensible, c'est-à-dire perceptible, visible, digne de préoccupations morales ou politiques, un problème qui ne l'était préalablement pas. D'autre part, rendre sensibles les individus interpellés afin qu'ils deviennent attentifs, réceptifs, portés à s'émouvoir et prompts à réagir" $(2009,20)$.

580 Boltanski 1993.

581 Voir aussi Chavot, Felt et Masseran 2001. 
la visibilité sociale de la cause du don d'organes. Ils déplorent ainsi la rareté des manifestations pour faire connaître le don d'organes auprès des citoyens, à l'instar de la Journée nationale du don d'organes; "ça devrait se faire plus qu'une fois par année quand même pour plus sensibiliser les gens, passer des films, faire des conférences-débats pour inviter le peuple suisse à venir y participer, mais ça il n'y a pas... le peuple, à mon avis, n'est pas suffisamment informé » (Thomas, AR, comité).

Pour la plupart des interviewés, le problème majeur réside dans le fait que les individus ne sont ni habitués ni incités à penser la question du don d'organes et celle de leur devenir après la mort; "peut-être qu'ils ne sont pas été assez préparés, c'est peut-être ça la cause, et j'ai toujours dit [...], comme on apprend le civisme, on devrait apprendre le don d'organes; donner, qu'est-ce qu'on fait de son corps, pour finir, on le brûle, est-ce que cela vaut la peine de le brûler? [...] il faut éduquer les gens" (Sylvie, AG, membre). Transformer le don d'organes en question citoyenne représente un travail en profondeur qui suppose d'agir sur la société et les mentalités. Dans cette logique, certains considèrent la problématique de la carte de donneur comme un épiphénomène; «il ne faut pas croire que c'est en faisant signer des cartes qu'on a gagné, non, loin de là, il y a toute une éducation à faire " (Bernard, AG, comité).

De par leur rôle majeur de relais de messages dans l'espace public ${ }^{582}$, les médias sont une cible significative des critiques des acteurs associatifs. Nombre d'entre eux expriment des avis mitigés concernant la place marginale que la presse a tendance à réserver au sujet du don d'organes. Sa médiatisation accrue serait l'un des moyens de sensibiliser plus intensément le public; "je ne pense pas que les gens vont changer de mentalité du jour au lendemain [...] c'est un truc où il faut taper sur le clou tout le temps, tout le temps! [...] la télévision, la presse, ce serait important de taper régulièrement sur le clou, ça c'est un fait » (Claude, AG, comité). Si la faible couverture médiatique est pointée du doigt, le traitement médiatique - la manière dont les journalistes en parlent - n'est pas

582 Hammer 2012. 
non plus épargné par les critiques. Marc qualifie de "pitoyable» le sensationnalisme qui à ses yeux est au fondement de l'approche journalistique du don d'organes, loin d'une démarche qui chercherait à informer ou faire réfléchir le public. Il fait notamment référence à des articles relatant des vols d'organes: «les journalistes, il faut faire des premières pages: "il s'est fait arracher son rein dans les toilettes publiques" [...] comme manière de promouvoir la cause, excusez-moi mais c'est de la merde ce que l'on entend et lit dans les journaux, c'est inacceptable» (FD, comité). Bien que critiquant cette logique sensationnaliste et commerciale, Nadia en a fait son parti, considérant qu'il s'agit d'un mal nécessaire pour atteindre une certaine visibilité en tant qu'association et par conséquent pour faire exister la cause; "si on n'a pas quelqu'un de célèbre... "Oh non, mais cela ne nous intéresse pas", il faut toujours avoir un nom... ça me dégoûte, alors des fois je dois aller lécher le c... des gens connus pour pouvoir avoir [une tribune médiatique]»(FD, comité).

Si l'intensification des campagnes d'information et de la médiatisation est mise en exergue comme moyen d'accroître la visibilité du don d'organes, l'ambition de normaliser le don d'organes dans les esprits s'exprime probablement le mieux en lien avec l'importance du milieu scolaire. Nombre d'acteurs associatifs préconisent une démarche beaucoup plus active de sensibilisation dans les écoles, qui symbolise cette préoccupation d'éducation en profondeur de la société; "s'il y avait une impulsion fédérale pour que les cantons aient l'obligation d'informer clairement et de prévoir deux fois dans l'année quelque chose, peut-être différencié en fonction de l'âge des enfants... comme beaucoup d'autres choses, lorsque les jeunes, les enfants sont informés, ils amènent l'information à la maison, eux suscitent la discussion et ils ont moins de réticences, ils sont plus ouverts souvent que les parents » (Pascal, AR, membre). La communication dans les écoles permettrait de combler le défaut de socialisation du don d'organes et de rendre ainsi le geste "presque naturel »; " [les gens] seront plus intéressés, plus réceptifs... c’est peut-être une solution » (Laurent, FD, membre). Pour Richard aussi, la pénurie d'organes est un problème d'éducation scolaire. À ses yeux, le don d'organes devrait être abordé en termes de charité 
et de valeur humaniste, dès lors que cette question fait partie du développement de chaque individu; "si on avait parlé autant de la mort que de la sexualité, on serait vachement plus au point» (AR, membre). Les jeunes, jugés davantage "réceptifs ", constituent un public cible; "ce qui est le plus porteur pour le don d'organes, c'est la jeunesse... c'est ceux-là qui vont nous faire avancer» (Bernard, AG, comité).

La normalisation du don d'organes comme enjeu social représente un objectif consensuel pour les acteurs associatifs rencontrés. Il s'agit de favoriser l'attention de la population en banalisant la thématique dans l'espace social afin que la réflexion individuelle sur le don d'organes devienne une démarche évidente et généralisée. Enraciner la question du devenir de son corps après sa mort dans la culture commune, en faire une banale question de société représentent une première fonction de la sensibilisation par l'information du public.

\section{Encourager le geste du don d'organes}

Tout en reconnaissant que les campagnes institutionnelles d'information peuvent avoir une utilité, nombre d'acteurs associatifs jugent qu'elles ne sont pas à même de convaincre les individus d'être des donneurs potentiels. Comme l'a montré le chapitre précédent, les critiques principales adressées aux messages de l'OFSP concernent leur neutralité, leur manque d'attractivité, et par conséquent leur inefficacité. Faisant le constat des limites de la politique d'information des autorités et de la pénurie persistante d'organes, ils sont nombreux à souhaiter une communication qui incite de manière plus vigoureuse et résolue le public à la réflexion et à devenir donneur potentiel. Dans les discours des acteurs associatifs, cette deuxième fonction de l'information publique tournée vers la promotion du don d'organes n'est toutefois pas homogène et se concrétise dans des conceptions parfois divergentes des messages adressés à la population.

\section{La rhétorique du besoin individuel}

Un argument plusieurs fois évoqué dans notre corpus afin de susciter une prise de conscience des individus consiste à renver- 
ser le raisonnement habituel - «êtes-vous d'accord de donner vos organes? »- en mettant en avant la situation hypothétique du besoin d'une greffe. C'est cette stratégie à laquelle Adeline indique parfois recourir lors de la tenue de stands dans l'espace public; "[on leur dit] "Mais écoutez si cela vous arrive à vous, votre enfant, votre mari"... je prends des exemples extrêmement concrets, il faut les sensibiliser les gens [...] les gens réagissent favorablement quand on leur dit: "Si cela vous arrive à vous, vous faites quoi ?", il faut personnaliser la manière de parler de cette question" (AG, comité). La personnalisation de la discussion sur le don d'organes consiste ainsi à ne plus actionner le ressort principal du don (l'altruisme) pour le remplacer par un autre impératif: la nécessité de bénéficier du geste d'autrui. Pour certains, la rhétorique du besoin personnel n'est pas assez mise en avant dans la communication publique: le message que tout un chacun peut un jour être concerné par une indication de greffe serait plus porteur. Interpeller le public en tant que receveur potentiel d'un organe (ou proche d'un malade dans le besoin) est envisagé comme un mécanisme de sensibilisation plus efficace que la figure du donneur ${ }^{583}$; "les enfants aussi, c'est vrai quand on pose la question, "vous devriez donner à votre fils pour qu'il survive", alors là on voit les choses complètement différemment " (Julie, AR, comité). Ce renversement de perspective permet d'enclencher la réflexion individuelle à partir d'une situation émotionnellement forte; "quand on prend le problème à l'envers... "Imaginez que c'est votre fils qui a besoin d'une greffe de cœur ou de foie, qu'est-ce que vous feriez?", “Ah ben, là il faudra vite qu'on le sauve, il faut qu'on lui donne un organe”... là d'un seul coup les gens en mettant le problème à l'envers: “Ah ben, on n'a jamais pensé qu'un jour on aurait besoin d'un organe" [...] "d'un seul coup, c'est votre fils qui va mourir, parce qu'il n'aura pas de greffe", alors à ce moment-là les gens disent: "Mais je vais aller écraser tout le monde pour sauver mon gamin" » (Murielle, FD, comité).

583 Cette rhétorique du besoin individuel pourrait être vue comme une forme ordinaire de l'argument épidémiologique qui consiste à souligner que statistiquement un individu a plus de chances d'avoir un jour besoin d'un organe que d'être en situation de donner un organe. 
Cette première modalité de la sensibilisation rejoint en partie le modèle de la réciprocité dans la mesure où la cohérence morale entre la disposition à recevoir et à donner est posée. On voit ainsi plus largement que, pour une partie des acteurs associatifs, des messages centrés sur le besoin individuel sont plus à même de rendre les individus "concernés" bien que le don soit un sujet abstrait et loin de leurs préoccupations concrètes. Davantage que l'appel à des valeurs altruistes, la personnalisation de l'enjeu permettrait de rendre les gens sensibles à la problématique du don d'organes.

\section{Promouvoir une image positive du don d'organes}

Montrer la transplantation sous un jour ouvertement positif constitue une deuxième modalité récurrente de l'encouragement au don d'organes. Pour plusieurs interviewés, ce sont les succès, les bienfaits de la greffe pour le receveur, le potentiel thérapeutique du don, le résultat spectaculaire et extraordinaire découlant du geste qui doivent être soulignés, plutôt que les circonstances dramatiques qui en sont la source. Mettre en avant le gain de vie(s) et les conséquences heureuses et salutaires que permet le don traduit une vision pragmatique de la communication publique: privilégier l'utilité et le bénéfice de l'action. "On parle trop du mort (ton insistant) et on ne parle pas assez de la chance... on peut sauver en tout cas cinq personnes par donneur! [...] on doit donner la publicité dans le sens que c'est une chance, une chance pour cinq personnes en principe de recevoir la vie!»(Gilles, AG, comité). Insister sur le côté positif du don de ses organes permettrait de sensibiliser le public, de toucher les consciences, d'interpeller l'individu hésitant ou réticent, voire de le convaincre à être un donneur potentiel. Montrer la vie plutôt que s'appesantir sur la mort, faire valoir le nombre de patients sauvés par un don, mais aussi l'utilité de la mort, c'est le type de message que prônent plusieurs acteurs associatifs: «on devrait être plus cru... montrer le positif des choses [...] qu'après [un don] on peut sauver huit vies... beaucoup de gens nous disent: "C'est vrai qu'après... une fois que je suis mort"... "Eh bien, avec votre corps, on peut sauver huit vies”, moi je pense que cela interpelle plus» (Stéphanie, AR, comité). 
Dans une logique similaire, certains interviewés évoquent l'ambivalence qui est au cœur du don d'organes (une vie renaît grâce à une mort) et la difficulté pour tout un chacun de se poser la question de sa propre mort. Ayant l'impression que le thème de la mort occupe une place importante dans le discours dominant sur le don d'organes, Sébastien regrette que cette ambivalence ne soit pas gommée, ou au moins atténuée, au profit de la vie dans la communication publique; "d'un côté, on prolonge la vie au maximum, la mort c'est presque une faute, presque une erreur [...] et d'un autre côté, on nous parle de mort à la place de don d'organes, il y a quelque chose de complètement antinomique, et ça donne une espèce d'interférence très forte » (AG, membre).

L'idée de promotion est explicite lorsque certains acteurs associatifs pensent la communication sur le don d'organes en termes de démarche publicitaire ou de stratégie marketing; "si vous voulez vendre un appareil de télé ou une bouteille d'eau minérale, il ne faut pas parler des risques, il y a peut-être des petits trucs radioactifs dans cette eau, il ne faut pas communiquer cela, il faut communiquer que c'est bon pour la santé »(Gilles, AG, comité). Pour Suzanne qui fustige la faiblesse des messages de l'OFSP, le sujet du don d'organes devrait être traité comme n'importe quel autre "produit » qu'il s'agit de «vendre» au public; «il faut peut-être employer des personnes compétentes qui vont comme dans le marketing pour des produits... quelque part c'est la même chose, on arrive bien à vendre de la lessive, c'est la même chose [...] si on veut encourager le don d'organes, il faut employer des mots flash [...] il faut intéresser la personne et que ce soit vivant et pas que ce soit gnangnan» (AG, membre). Cette vision de l'encouragement au don s'appuie sur la valorisation des aspects positifs de la transplantation, centrée sur la figure du bénéficiaire du geste, plutôt que sur le contexte douloureux associé à la figure du donneur.

\section{Bousculer les consciences, montrer la souffrance}

Parmi les acteurs associatifs qui se montrent virulents dans leurs critiques de la communication sur le don d'organes en Suisse 
ces dernières années, quelques-uns se distinguent par des prises de position très marquées, prônant des messages forts destinés à bousculer les consciences; "il faut montrer et peut-être plus choquer, et bien voilà, une personne est décédée mais cela peut sauver huit vies » (Julie, AR, comité).

Les propos de Christophe illustrent cette conception d'une sensibilisation qui cherche davantage à persuader le public qu'à le convaincre, en recourant à des contenus émotionnels qui touchent au cœur les individus, "qui devraient vraiment secouer les gens [...] qui réveilleraient les gens au fond d'eux!» (FD, membre). Pour sortir l'individu lambda de l'indifférence, Christophe imagine des messages choquants et réalistes mettant en scène le contexte dramatique de situations de don et de non-don; «il faut faire quelque chose de plus marquant, il faudrait vraiment faire une situation où l'on se retrouve face à une personne au CHUV, par exemple face à un coordinateur de transplantation, et une maman en larmes avec le coordinateur qui lui demande s'il peut prélever les organes de son fils, en urgences qui se rapproche du réel! [...] il faut vraiment une personne en train de mourir qui dit dans l'oreille à quelqu'un après un accident de la route: "J'aimerais donner mes organes, il faut le dire aux ambulanciers”, et après une situation où la famille n'est pas d'accord, montrer la personne qui est mourante, qui a reçu un coup sur la tête et qui est en train de partir et qui arrive à dire: "Mes organes je les donne", et après la famille qui vient au CHUV et qui dit qu'elle est contre, et finalement la personne ne peut pas donner ses organes parce que la famille était contre et qu'elle était pour, donc voilà c'est un peu ambigu comme situation » (FD, membre). Comme d'autres, Christophe dénonce le manque de médiatisation de la problématique du don d'organes et la «frilosité » des journalistes. À ses yeux, les malades ne sont pas suffisamment présents dans les médias, alors qu'ils sont précisément les mieux placés pour montrer au public la douleur, l'épreuve pénible de l'attente d'un organe, le besoin vital d'une greffe, mais aussi les conséquences fatales de la pénurie; "il faudrait plus médiatiser tout ça en fait, il faudrait aller vers un jeune de 8 ans par exemple qui est sur une liste d'attente et qui est sur le point de mourir et puis que finalement par manque 
d'organe, ce gamin, il est mort, et cela aussi ça arrive, et ça on n'en parle pas, il faudrait plus parler des gens qui meurent» (FD, membre). Alors que Gilles (AG, comité) affirmait que l'« on parle trop du mort et [...] pas assez de la chance [que représente un don]» dans une volonté de dédramatiser la communication sur le don d'organes, la perspective défendue par Christophe consiste au contraire à heurter la cible des messages et à infliger au public la face sombre ou tragique du don d'organes pour le faire réagir. À l'inverse d'une promotion de la cause du don d'organes fondée sur les aspects positifs de la transplantation, cette troisième modalité repose avant tout sur des émotions fortes qui soulignent la souffrance, la mort, ou encore les difficultés concrètes de la décision du don.

\section{Des positions nuancées sur la promotion}

Qu'elle soit axée sur le pathos ou sur des messages positifs, la conception explicitement promotionnelle du don d'organes ne fait pas l'unanimité dans notre corpus. C'est le cas notamment de Jean, l'un des rares à approuver la brochure de l'OFSP, qui s'oppose à une stratégie d'information du public inspirée par le marketing: «Mais on donne la possibilité de choisir, et puis ce n'est pas de la vente de marchandise, parce que dans la vente, on ne voit jamais que l'on dise que cela a des limites, c'est toujours : notre produit est le meilleur, il a ceci ou cela... maintenant là on laisse la possibilité [de choisir]»(AR, comité). Quelques autres acteurs associatifs défendent une information "objective» et équilibrée susceptible de favoriser la réflexion de l'individu. Pour Didier, par exemple, il ne faut pas recourir à «la langue de bois » et occulter les aspects moins positifs de la transplantation; "il faut être transparent dans ce que l'on dit et pas avoir peur d'évoquer certains aspects aussi du don » ( $\mathrm{AR}$, comité), tels que les phénomènes de rejet, les échecs possibles de la greffe, ou encore les effets secondaires des traitements immunosuppresseurs. À ses yeux, il est important de donner une image de la médecine de transplantation fidèle à la réalité tout en tenant un discours favorable à son égard. Il s'agit ainsi de parler «du don d'organes de façon brute» et de répondre aux interrogations des 
gens qui ne sont "pas stupides" (Didier, AR, comité). De manière similaire, quelques interviewés vantent les qualités d'un reportage télévisé ${ }^{584}$ sur la transplantation montrant certaines de ses facettes moins heureuses; "on a vu aussi la personne qui refusait et puis... non, c'était très bien. Et la personne où ça a loupé, où cela ne marche pas parce que c'est aussi quelque chose qui doit être dit... non, moi, j'ai trouvé très bien alors qu'ils fassent ça » (Léo, AG, membre); «on voit les gens en attente, on voit même les gens qu'on n'est pas sûr de prendre, parce qu'ils sont mentalement pas capables ou parce qu'ils picolent trop... c'est bien qu'on montre ça, mais on voit l'engagement des infirmiers, des médecins, des coordinateurs...» (Patrick, AG, membre). Ces propos reflètent le souci d'une information équilibrée qui montre les différents enjeux du don d'organes, la complexité de la greffe, ses bienfaits majeurs sans voiler pour autant les complications et les difficultés.

D'autres interviewés défendent une politique d'information du public centrée sur la liberté de choix et la réflexion individuelle, condamnant tout message susceptible de culpabiliser l'individu non donneur ou non concerné. C'est le cas de Laura, l'une des rares à juger favorablement les campagnes de l'OFSP; "où il y a les gens qui disent, "moi je suis pour", "moi je suis contre"... parce qu'il y en a qui disent: "Non, moi je suis contre, ça m’appartient!”... et après, ils disent: "Ils ont tous raison", moi je vais assez dans ce sens-là, chacun sa réflexion... [...] pour moi cette phrase [...] cela reflète la réalité, on ne peut pas obliger, c'est quelque chose qui est vraiment très personnel, je suis étonné d'entendre que les autres ne trouvent pas bien ces spots [...] je trouve que justement ça pousse peut-être les gens à réfléchir $[. .$.$] cela ne culpabilise personne » (AR, comité).$ La rhétorique du besoin personnel, évoquée précédemment comme stratégie de sensibilisation, est contestée par certains acteurs associatifs car elle est susceptible de stigmatiser les individus réticents ou opposés au don; "c'est contre-productif, c'est pas comme cela qu'il faut aborder le thème du don d'organes, ce n'est pas en disant: “si cela vous arrivait à vous...”... en faisant cela, vous l'attaquez,

584 Il s'agit de La Part de l'autre, reportage en cinq épisodes sur la transplantation d'organes tourné aux HUG, diffusé au printemps 2013 à la Télévision suisse romande. 
vous le culpabilisez déjà, donc il va partir, mais il faut répondre aux questions et accepter que quelqu'un dise je suis contre, on doit l'accepter [...] chaque individu a le droit de dire: "Non, je ne suis pas d'accord" »(Didier, AR, comité). D'autres expriment leur scepticisme quant au bien-fondé de démarches militantes trop ouvertement en faveur de la cause du don d'organes, préférant des approches prudentes, respectueuses de l'opinion de chacun; "il ne faut pas non plus que les personnes aient le sentiment qu'on veuille les convertir, c'est pas du tout ça [...] il faut respecter aussi la décision, la personne et ça, c'est essentiel, on n'est pas des ayatollahs... je ne fais pas l'apologie du don [...] je suis très respectueux de toutes les décisions »(Sébastien, AG, membre).

\section{La force du témoignage : I'incarnation du problème}

La normalisation du don d'organes comme question de société, d'une part, et l'encouragement des citoyens à prendre position sur la question ou à se déclarer donneurs, d'autre part, correspondent à deux visées majeures de l'amélioration de l'information du public. Si la responsabilité d'assumer ces deux fonctions revient principalement aux pouvoirs publics, elle revient aussi en partie aux acteurs associatifs par le biais du témoignage personnel. Cette troisième fonction de l'information du public renvoie spécifiquement à un registre d'action assumé par les personnes transplantées elles-mêmes.

Pour la grande majorité des interviewés, témoigner de son expérience individuelle peut contribuer à une plus grande prise de conscience du problème du don d'organes. Plusieurs soulignent la nécessité de sensibiliser la population à partir d'exemples concrets de patients et mettent en avant leur propre parcours de transplantés lors de discussions avec des personnes hésitantes, voire réfractaires. Pour certains acteurs associatifs, les témoignages des personnes greffées sont la voie royale en matière de promotion du don d'organes, notamment lorsque les ingrédients essentiels du geste du don sont présents (le besoin d'organe, l'altruisme); "il y avait un type génial qui a donné son rein à sa femme, ça c'est le genre de type qui devrait arrêter de travailler et parcourir la Suisse pour expliquer ce 
qu'il a fait [...] comment il a raconté son histoire, moi j’ai trouvé cela fantastique, ce type, on devrait le mettre à la télé dix minutes tous les soirs (rires) en Suisse allemande, je suis sûr qu'il aurait de l'effet parce que là il a eu de l'effet [sur la salle dans laquelle il était intervenu], c'est cela qui manque [...] il a simplement résumé son histoire sans plus, il avait quelques photos de lui et de sa femme à l'appui de son discours [...] ce type-là, vous le mettez en Suisse allemande, je suis sûr qu'il double [le nombre de donneurs] en peu de temps! [...] ce genre de témoignage, c'est parfait... [...] il était prenant, il était convaincant, il était rassurant, voilà! »(Pierre, AR, comité).

Le potentiel de sensibilisation du public par les témoignages de patients repose notamment sur l'exemplarité des greffés de l'efficacité de la transplantation. Ils sont la preuve manifeste que le don permet de sauver une vie, a fortiori de qualité. Ils sont en même temps la preuve du besoin d'organes et du problème de la pénurie. Le chapitre suivant montrera que le témoignage constitue une compétence et une prérogative fortes revendiquées par la plupart des associations. S'exprimer en public sur son parcours de malade, sur l'expérience du don et de la greffe fait en effet partie des activités phares des associations auprès de la population. La parole des transplantés incarne la puissance thérapeutique de la technique médicale et devrait aider à convaincre à moyen terme la société du bien-fondé de la transplantation; "[la société] est en phase d'être prête, c'est un cheminement, c'est un processus qui prendra du temps et c'est par l'exemple de gens comme moi, je ne suis pas le seul, qui sont les meilleurs vecteurs de la réussite et de la nécessité du don » (Didier, AR, comité). Pour Sébastien, qui préconisait une communication plus adaptée en direction des citoyens («il faut juste bien leur parler, il faut leur parler juste, sans idéaliser»), les témoignages personnels sont précisément à même de toucher les gens, d'attirer leur attention sur une situation problématique, voire d'influencer leur disposition au don d'organes; "peut-être donner plus la parole aux personnes transplantées qui peuvent dire aussi: "Voilà, j’ai reçu cet organe, c'était in extremis, et puis si cette personne-là ne s'était pas questionnée sur le don d'organes à un moment donné, eh bien, ça aurait été très problématique” " (AG, membre). 
Convaincus de la force de sensibilisation des témoignages, les acteurs associatifs n'ont cependant pas tous la même définition du «bon» témoin, c'est-à-dire du promoteur adéquat et efficace de la transplantation. Autrement dit, quel doit être le profil du transplanté qui saura valoriser le geste du don d'organes et délivrer une image positive de la greffe ? À cet égard, Nadia a une conception bien définie des caractéristiques requises. À ses yeux, les personnes transplantées engagées dans la communication doivent être attractives par leur apparence et surtout emblématiques des bénéfices de la greffe par l'activité physique qu'elles déploient. Il s'agit typiquement de patients transplantés jeunes, qui revivent pleinement après avoir échappé de peu à la mort, qui mènent une vie normale, sont épanouis, font ou refont du sport et qui fondent une famille. N'importe quel transplanté n'est dès lors pas un bon «ambassadeur» du don d'organes; «si on est un vieux monsieur qui a toujours bu et mangé du fromage et blablabla qui a un problème cardiaque, on est moins... que si on voit Clarisse ${ }^{585}$ qui avait peut-être 16, 17 ans, magnifique fille, vraiment super belle [...] elle est prof d'école... et du jour au lendemain, quelque chose comme ça arrive, et puis elle renaît, et elle fait du sport, et elle est positive, elle est rayonnante, cette fille, alors pour moi, elle est une bonne ambassadrice» (Nadia, FD, comité). Selon leur âge, leur style de vie, leur état de santé et leur apparence, les patients transplantés sont inégalement dignes de représenter publiquement le don d'organes ${ }^{586}$. Le comédien Gérard Depardieu, greffé hépatique à l'hygiène de vie peu scrupuleuse, apparaît comme l'antithèse de Clarisse, dont la médiatisation ${ }^{587}$ risque de desservir

585 Pseudonyme d'une patiente greffée, ayant de nombreux succès sportifs à son actif, et membre d'une association généraliste.

586 D'autres traits composent l'image sociale et morale du transplanté, tels que la gratitude ou la reconnaissance envers le donneur (ou sa famille), ou le respect envers l'organe reçu (Sharp 2006). L'enjeu du "bon" ou "mauvais" témoin n'est ainsi pas négligeable. Biaudet $(2013,283)$ relate ainsi qu'au sein d'une association en France "l'activité de témoin est contrôlée" dès lors que "la parole [des personnes transplantées] mérite d'être diffusée et d'autres dont le discours requiert d'être tu, ou au moins encadré", notamment lorsque le registre de la plainte des conditions de vie après une greffe est trop présent.

587 Voir, par exemple, un article paru le 27 janvier 2013 dans Le Matin www.lematin.ch/sante/sante/On-peut-vivre-normalement-apres-une-greffe-du-foie/ 
la cause du don d'organes; "ce n'est pas une bonne publicité [...] on a un truc comme ça, c'est un peu comme une grosse merde qui arrive au milieu de tout le travail qu'on a fait, ça fait vraiment de la contre-pub» (Nadia, FD, comité).

Sportif, beau, actif, épanoui... quelques interviewés ne partagent pas cette définition du bon témoin à tous points de vue pour plaider la cause du don d'organes. Jean notamment regrette la tendance à faire moins cas des parcours des patients ordinaires, par exemple dans les journaux internes des associations; "personnellement j'ai toujours trouvé dommage que l'on mette au premier plan des sportifs... pourquoi on ne mettrait pas une fois la vie de famille d'une mère qui a été transplantée, comment elle peut élever ses enfants, qu'est-ce qu'elle apporte [...] ou une jeune fille qui a été transplantée et qui a eu toutes les difficultés d'étudier, et puis que maintenant elle peut continuer à étudier, et puis, eh bien, ça aussi on n'en parle pas, mais on parle de celui qui a réussi à faire tant d'exploits » (AR, comité). Néanmoins, il rejoint la position de Nadia concernant le potentiel inégal de sensibilisation des patients transplantés selon leurs caractéristiques sociales; «je me suis aperçu que le témoignage d'une personne d'un certain âge n'apporte plus, il faut trouver des jeunes qui ont été transplantés et porter le témoignage de ces jeunes, sinon ça ne sert à rien!» (Jean, AR, comité).

La distinction entre les figures «exceptionnelle» et «ordinaire» des patients pour représenter la réalité positive du don d'organes dans l'espace public fait l'objet d'un discours beaucoup plus incisif de la part de Charles. Il critique sévèrement la construction publique de la figure du transplanté héroïque, dans sa version sportive; «il y a ce petit supplément qui, d'ailleurs, est exploité de manière complètement imbécile maintenant, il faut aller sur l'Everest pour faire juste... des conneries » (AG, membre). S’il dénonce la logique sensationnaliste de la médiatisation du don d'organes qui à son

story/29401863, ou cet extrait tiré d'un article d'un journal français: "Ce nouveau foie, Depardieu ne l'a pas ménagé ces dernières années, multipliant les incidents liés à des repas trop arrosés, tout en enchaînant les films à marche forcée, tandis que son tour de taille s'élargissait" (Le Point, 9 janvier 2013, en ligne: www.lepoint.fr/culture/ les-exces-de-gerard-depardieu-inquietent-ses-proches-09-01-2013-1611232_3. php). 
avis participe de cette situation, il pointe également du doigt une part de connivence de certains acteurs associatifs; «il y aurait [...] une demande à exprimer aux associations de patients pour leur demander de faire autre chose que du spectacle promotionnel pour que les gens donnent [leurs organes]»(Charles, AG, membre). Au lieu de mettre en avant la «performance physique» du transplanté comme symbole de l'efficacité de la greffe, il préconise le marqueur social du « retour à l'activité » qui à ses yeux est plus représentatif de l'expérience de la plupart des patients transplantés et des difficultés rencontrées, contrairement à la dimension sportive et à la capacité physique de l'organisme.

Deux logiques différentes du témoin «exemplaire» de la promotion du don d'organes se dessinent ici. La première renvoie à une préoccupation centrée sur l'exceptionnalité et le spectaculaire qui forcent l'admiration du destinataire du message, à travers le corps et l'exploit sportif. Cette conception du témoignage qui privilégie les aspects positifs de la greffe peut s'accommoder d'une forme de surenchère dans la normalité de la qualité de vie retrouvée. La seconde logique du «bon» témoin de la transplantation vise certes à souligner les bénéfices de la greffe, mais en cherchant surtout à aborder la réintégration des rôles sociaux quotidiens et à représenter des vies ordinaires, plus proches du citoyen lambda et donc plus susceptibles de le toucher.

L'importance des témoignages des patients transplantés s'inscrit plus largement dans une tendance forte au traitement personnalisé des questions de santé publique et reflète une «subjectivisation de la délibération sociétale [qui] conduit à la promotion du témoin érigé en acteur central de l'espace privé/public ${ }^{\mathbf{5 8 8}}$ ». Vecteurs singuliers de l'authenticité d'un problème collectif autant que de la légitimité d'une pratique médicale et sociale, les témoignages portent le «vrai» dans leur dimension factuelle ou affective. À ce titre, on leur attribue souvent la faculté de relier la sphère privée et la sphère publique, d'établir une "filiation avec l'ensemble du corps social ${ }^{589}$ ", et donc

588 Mehl 2003, 492.

589 Idem, 493. 
de produire l'identification du public. Si le témoignage profane peut servir d'illustration d'un fait général ou tenir lieu d'argument en soi ${ }^{590}$, il peut aussi, particulièrement dans le don d'organes, être qualifié de témoignage "performatif» dès lors que le récit du transplanté vise à engager autrui dans la disposition au don ${ }^{591}$.

\subsection{Conclusion}

Si le problème de l'information est érigé comme l'une des causes majeures de la faible réceptivité du public dans le contexte actuel, l'amélioration de la politique d'information est envisagée comme une mesure prioritaire. L'importance du thème de l'information de la population dans les discours des interviewés tient au fait que la communication de proximité est l'une des activités principales des associations, domaine dans lequel elles revendiquent des compétences et une expérience que les autres acteurs du don d'organes n'ont pas (voir chapitre 9). Mais elle tient aussi à la définition des acteurs associatifs de la situation, caractérisée par une vision optimiste des attitudes du public vis-à-vis du don d'organes. Globalement, les acteurs associatifs font état d'un climat moral plutôt favorable au don d'organes au sein de la population. La majorité des Suisses sont perçus comme des donneurs potentiels mais dont la décision est latente précisément en raison d'une politique d'information déficiente sur plusieurs points. Sur le plan cognitif d'abord, une meilleure information du public suppose de diffuser des connaissances factuelles et scientifiques sur la médecine de transplantation afin de corriger des représentations erronées, de réduire les peurs et de limiter le poids de l'imaginaire social et des fantasmes sur l'échange d'organes, notamment autour de la mort cérébrale. Sur le plan sociétal ensuite, il s'agit de normaliser la question du don: l'information devrait servir à généraliser la prise de conscience sociale de l'importance du don d'organes. Construire progressivement une culture du don d'organes grâce à l'éducation de la population doit permettre

590 Mehl 2003.

591 Amey et Hammer 2010. 
de banaliser la réflexion sur le sujet. Ce travail de fond peut être rapproché de la quête d'une «compatibilité culturelle ${ }^{592}$ ». Enfin, les acteurs associatifs soulèvent une interrogation cruciale: quel doit être le but recherché de l'information du public? La promotion est la réponse prédominante: encourager les individus à se déclarer donneurs potentiels. C'est dans cette optique que les préoccupations des interviewés sont les plus vives autour du manque de réceptivité de la population et de l'efficacité des messages. Certes, les frontières entre l'incitation à la réflexion, la sensibilisation au problème de la pénurie et à l'importance du geste, et l'incitation à signer une carte de donneur sont parfois ténues et difficiles à trancher dans les propos des interviewés. Pour autant, la variété des fonctions attribuées à l'information renvoie à des questions majeures sur la signification sociale et morale du don d'organes : s'agit-il d'un devoir citoyen, d'un geste attendu de tout être humain envers son prochain ? S'agit-il d'un choix comme un autre dans lequel être "pour» et être «contre» ont la même valeur? Et à quelles conditions le refus est-il acceptable? Ou encore, dans quelle mesure est-il légitime de porter un jugement sur chacune de ces options? Le choix politique de la neutralité en matière de communication sur le don d'organes réactive plus que jamais ces questions centrales.

Les positions exprimées par les acteurs associatifs - quoique certains n'aient pas de position affirmée - reflètent une diversité de conceptions (oscillant entre le don comme obligation morale et comme liberté individuelle) et d'attitudes vis-à-vis des résistances au don. L'une des tensions principales oppose une politique d'information neutre, peu engagée et axée sur le principe de l'autodétermination, à une politique active d'information explicitement favorable au don d'organes, recourant au besoin à des messages émotionnels susceptibles de briser l'indifférence du plus grand nombre. Il n'y a donc pas de consensus fort parmi les acteurs associatifs sur la définition du don d'organes comme enjeu de communication publique, si ce n'est autour de quelques positions générales. Néanmoins, la légitimité de la présentation du geste du don d'organes comme

592 Moulin 1995. 
enjeu d'un débat contradictoire est très marginale dans notre corpus - les critiques virulentes de la brochure de l'OFSP l'attestent. La publicisation d'opinions défavorables au don peut en effet être lue comme «la construction du "don d'organes" non pas comme objet de consensus social mais comme objet de positionnements contradictoires, autrement dit comme un objet de débat ${ }^{593} »$. Interroger publiquement le caractère a priori consensuel du don d'organes, qui revient d'une certaine manière à le relativiser socialement, constitue une inflexion politique et axiologique majeure pour les acteurs associatifs. Si ces derniers condamnent souvent de manière virulente et sans ambages le contexte social et politique en Suisse, cela ne signifie pas forcément qu'ils expriment publiquement ces critiques ou que celles-ci sont au cœur des activités concrètes de leurs associations, sans compter que les divergences dans la définition du don d'organes dans notre corpus ne recoupent pas nécessairement la diversité de l'espace associatif. Il convient donc de s'interroger sur les actions, revendications et stratégies des associations afin de mieux cerner leur rôle. 


\section{Chapitre 9}

\section{La mobilisation des associations : soutenir les malades et le don d'organes}

Raphaël Hammer et François Kaech

Après avoir décrit de quelle manière les acteurs associatifs perçoivent le don d'organes en tant que problème public, il convient de s'interroger sur leurs actions menées concrètement et sur le sens de leur engagement. Les buts affichés par les associations (dans leurs statuts, leur journal interne ou sur leur site internet) ne donnent qu'une indication générale de leur mission et sont insuffisants pour comprendre de près leurs domaines d'action et le rôle qu'elles jouent. La prise en compte de l'expérience des responsables et membres d'associations est nécessaire à un double titre. D'abord parce que les activités effectivement accomplies ne correspondent pas forcément aux buts formels de l'association. Quelle qu'en soit la raison, il peut y avoir un écart, plus ou moins important, entre l'ambition ou l'idéal qui fonde la raison d'être d'un collectif et ce que ce dernier met réellement en œuvre. Ensuite, les propos des acteurs associatifs revêtent un double statut puisqu'ils peuvent s'exprimer comme porte-parole du collectif et/ou à titre individuel ${ }^{594}$. Les données d'entretiens permettent ainsi de documenter ou compléter des informations relatives à l'association en tant que telle (mode de fonctionnement,

594 Buton 2005. 
composition, activités...), mais donnent aussi accès au point de vue de l'interviewé sur le rôle joué par son association. Cette dualité est d'autant plus pertinente dans notre cas que les objectifs et activités des associations ne concordent pas nécessairement avec la manière dont nos interlocuteurs situent les enjeux actuels de la cause du don d'organes (voir chapitres 7 et 8 ).

Ce chapitre analyse dans un premier temps les stratégies et actions associatives concrètement réalisées, sans chercher à en rendre compte de manière détaillée pour chacune des associations, ni à les évaluer à l'aune des buts affichés. Dans un deuxième temps, nous examinerons le rôle joué par les associations par rapport à la problématique du don d'organes en considérant les obstacles qu'elles rencontrent et les limites de leur capacité d'action.

\subsection{Stratégies et activités}

La diversité des activités réalisées par les associations renvoie schématiquement à trois grands objectifs: soutenir les patients sur le plan psychosocial et leur apporter une aide matérielle; informer et sensibiliser le public sur le don d'organes; et améliorer la prise en compte du don d'organes dans les politiques publiques ${ }^{595}$. Alors que l'aide (premier objectif) implique surtout des activités et des prestations tournées vers les membres du collectif, l'information du public et la mobilisation au niveau politique supposent des actions dans la sphère publique. Loin d'être exclusifs, ces objectifs sont souvent complémentaires. Cela est particulièrement vrai pour les deux premiers puisque nombre d'associations sont engagées à la fois sur le soutien aux membres et sur la promotion du don dans la société - certaines mettant la priorité sur l'un ou l'autre type d'activités.

\section{Le soutien aux membres}

«Notre devoir c'est d'accompagner ces gens qui sont transplantés, ils peuvent venir chez nous avec leurs problèmes, on peut discuter, on peut donner des informations [...] si on arrive à aider quelqu'un, 
c'est tout ce qu'il faut " (Gilles, AG, comité). L'aide aux membres se présente sous des formes diverses et répond à des attentes de nature psychosociale, voire identitaire, d'informations pratiques ou encore de soutien matériel et financier.

\section{L'entre-soi et le soutien psychosocial}

Une fonction primordiale de la vie associative consiste dans l'organisation d'activités favorisant l'entre-soi, c'est-à-dire la rencontre de personnes partageant une même condition et ayant traversé des épreuves similaires. Faire partie d'une association permet de se retrouver entre patients transplantés mais aussi entre patients traités en dialyse, une partie d'entre eux étant inscrits sur la liste d'attente pour une greffe. Si le développement des liens sociaux est relevé par la majorité des associations, il est plus particulièrement revendiqué au sein des associations de patients insuffisants rénaux et transplantés. Ce président affiche «le bien-être de nos membres » comme une priorité qui implique disponibilité d'écoute et sollicitude: "dans les relations que l'on entretient avec les patients qui sont nos membres, en termes de soutien psychologique, de soutien des conjoints, des familles... s'il y a quelqu'un qui me dit [...] "Il y a quelque chose qui ne va pas ces temps, est-ce que je peux venir te voir?", alors bien sûr et on discute, alors ça on fait » (Jean, AR, comité). Parfois, les responsables de quelques associations prennent les devants et rencontrent des patients non membres afin de se faire connaître et de connaître leurs besoins éventuels, par exemple en rendant visite aux malades dans les centres de dialyse.

Dans cette logique d'entraide, la plupart des associations mettent régulièrement sur pied des activités permettant aux membres de partager leurs expériences et de se divertir à l'occasion d'excursions, de sorties culturelles, de repas, etc. - activités qui ont lieu lors des assemblées générales mais aussi à des moments spécifiques; "on se retrouve au restaurant, on parle ensemble, c'est quelque chose de très intime [...] et l'apéritif c'est aussi un endroit où l'on peut parler de ses problèmes et échanger nos expériences, par exemple autour des médicaments, cet aspect d'échange a une grande importance» 
(Gilles, AG, comité). L'un des membres évoque le côté "rassurant» de pouvoir discuter avec d'autres patients de leur vécu de la transplantation, qu'il s'agisse de l'aspect somatique, psychologique, professionnel ou encore conjugal: "Au début on est assez content de faire partie de... on rencontre des gens qui ont déjà quelques années, comment ils vivent ça et... savoir s'ils ont les mêmes petits inconvénients qu'on a... c'est réconfortant... des choses comme ça, et puis les accompagnants... le vécu d'un conjoint de transplanté est plus dur que celui du transplanté, beaucoup plus dur» (Patrick, AG, membre). À l'occasion de ces activités de sociabilité, l'entre-soi et l'entraide se nourrissent mutuellement. Exemple parmi d'autres, le compte rendu de l'une des «rencontres automnales régionales» témoigne de l'importance des relations interpersonnelles pour la «culture» et l'identité de l'association:

«Les nouvelles amitiés se nouent puis se cultivent par le dialogue, l'échange d'expériences, le temps offert aux autres, la convivialité, la joie de se retrouver, l'écoute, le fait de s'insuffler mutuellement courage et confiance, de montrer de la joie de vivre et de la reconnaissance et surtout par le rire. Ce sont parfois de petits riens qui font le plus d'effet. Quel bonheur pour un membre de l'association de longue date qui ne se porte pas bien de recevoir une carte signée de tous qui lui redonne courage et envie de vivre, de voir que l'on pense à lui en tout temps. Quel réconfort pour le partenaire d'un membre défunt d'être également invité aux manifestations de l'association et d'être entouré dans son deuil ${ }^{596}$."

Le récit de l'un des interviewés - un transplanté d'une trentaine d'années - illustre la diversité des attentes et des formes de soutien liées à l'appartenance d'une association. Il met d'abord en exergue le bénéfice qu'il tire de l'expertise d'autres transplantés; «il y a le partage des informations... essentiel! Parce que... qui sait mieux qu'un transplanté pour nous donner ce genre d'information? Très certainement pas le corps médical, jusqu'à un certain point pour tout ce qui est très pointu, là $\mathrm{OK}$, aucun doute, mais pour tout ce qui est quotidien... je fais un petit coup de fil, voilà : "Comment tu ferais à ma place?”, ne serait-ce que pour des questions d'assurance ou

596 Bulletin de l'AST, $n^{\circ}$ 38, novembre 2011, 5-7. 
beaucoup plus...» (Sébastien, AG, membre). Le savoir des patients couvre aussi des aspects liés aux stratégies thérapeutiques et à la prise en charge médicale; "cela permet de croiser aussi les regards et cela ouvre à de nouvelles perspectives... (Sébastien, AG, membre). Au-delà du soutien pragmatique, Sébastien souligne également des liens précieux de solidarité les uns envers les autres; "il y a un suivi aussi... ça, c'est super, il y a vraiment un filet qui fait du bien... on sait que lui va un peu moins bien, alors on se soucie, on lui lance un coup de fil, le transplanté rénal ici à Genève, il va un peu moins bien en ce moment... ce côté humain fait énormément de bien, une fois de plus il supporte... c'est quelque chose sur lequel on s'appuie.» Le même interviewé montre que l'association de transplantés peut s'apparenter à une communauté basée sur des valeurs fortes de compréhension mutuelle et d'empathie réciproque: "C'est pas facile! Parce que c'est quand même une atteinte... on a traversé des étapes difficiles, et qui peut mieux comprendre qu'un transplanté? Il y a beaucoup d'estime et de réciprocité, de respect [...] c'est quelque chose en arrière-fond qui fait beaucoup de bien de savoir qu'il y a cette association et les membres.»

Enfin, Sébastien évoque l'identification aux pairs ${ }^{597}$ qu'offre le cadre associatif: pouvoir évaluer sa "normalité ", son état de santé, ses capacités et sa situation professionnelle en se comparant à autrui. "Se dire... dans quelle mesure les gens sont limités ou pas dans leurs activités au quotidien, est-ce que celui-là il travaille, est-ce que celui-là il ne travaille pas [...] j’ai une rente à l'AI à $50 \%$ et je pense que ce sera toujours comme ça, même si cela va extrêmement bien, mais cela permet de se positionner aussi... est-ce que je suis dans le faux, est-ce que je suis dans le juste... ne serait-ce que se comparer [...], comparer des trajectoires de vie et de dire: "Ah, il arrive à faire tout ça, eh bien, je devrais en être capable", ça c'est quelque chose que je ne retrouve nulle part ailleurs. " Le cas de Sébastien montre parfaitement que l'entraide peut être un besoin latent ou une attente forte des membres qui se concrétise essentiellement à travers la participation à la vie associative.

597 Colinet 2013. 
Le soutien psychosocial en réponse à des demandes individuelles constitue rarement une activité structurée et officielle des associations. Si certains interviewés participent à des groupes de parole organisés dans le cadre de l'association, c'est le plus souvent de manière informelle et occasionnelle que les acteurs associatifs apportent une aide morale à des patients redoutant la perspective d'une transplantation ou vivant difficilement l'attente d'un nouvel organe. Ce transplanté évoque le cas d'un malade indécis face à l'opportunité de se faire opérer; "on a discuté une heure, une heure et demie, le temps qu'on était là-bas [à l'hôpital] [...] ce qu'on a traversé, et ouvertement on lui dit aussi le pour et le contre, je lui dis, il y a ces médicaments, il faut faire attention, après tu auras une vie plus hygiénique pour être mieux, pour ton bien et tout ça [...] à la fin il s'est fait opérer, il m'a revu, il m’a dit “merci” " (Carlo, AG, membre).

\section{Le sport: un repère de normalité individuelle}

Les activités sportives jouent un rôle particulier dans le soutien psychosocial que recherchent certains membres ${ }^{598}$. Outre les rencontres interpersonnelles, le divertissement et les voyages, l'intérêt du sport réside dans les bénéfices médicaux d'une activité physique régulière pour la récupération et le maintien en forme après une transplantation ${ }^{599}$. La pratique du sport dans un cadre associatif, avec des pairs, constitue aussi une source de motivation pour reprendre ou commencer une activité physique. Pour certains, il s'agit d'une opportunité de se prendre en charge; "ça m'a permis de continuer une activité sportive, plutôt que de se laisser aller, parce que des fois il faut quand même un peu se bousculer [...] c'est des objectifs [les compétitions], ça nous permet de rester en forme, enfin, pour moi » (Sylvie, AG, membre).

598 Rappelons que la section sport de l'AST est spécialisée dans l'organisation d'activités sportives et de compétitions internationales réunissant des participants transplantés. D'autres associations proposent également des activités en lien avec le sport ou l'exercice physique mais à une échelle plus locale et ponctuelle.

599 Sur le plan médical, l'activité physique contribue notamment à entretenir la santé du nouvel organe et à réduire les effets secondaires liés aux médicaments immunosuppresseurs, tels que la prise de poids. 
Un autre bénéfice majeur souligné dans les entretiens réside dans la construction d'une identité positive de soi-même. Au-delà de la pratique du sport en tant que telle, l'ambiance et le profil des personnes rencontrées sont mis en avant. L'entre-soi des activités sportives permet d'échapper en partie à l'identité de «patient» dès lors que la condition de transplanté n'est pas au centre des discussions; " on ne parle pas tous de greffes, certains, oui, mais pas tous, c'est quand même plus ouvert...» (Sylvie, AG, membre). Pour Sylvie comme pour d'autres, pouvoir côtoyer des transplantés actifs, en bonne santé, dans le cadre des compétitions constitue une forme d'espoir et une parenthèse dans l'univers quotidien de la maladie; "ce sont des gens qui ont fait des choses, ils ne se sont pas repliés sur eux-mêmes [...] ça donne un coup de pouce pour se relever de ce carcan [...] alors [c'est] tout positif. » Le contexte du sport évacue pour un temps les aspects médicaux, les difficultés psychosociales et les soucis de santé. Il représente un horizon encourageant du sort de transplanté et agit comme une émulation; "cela me tire vers le haut, ça me tire de voir tous ceux qui ont participé, des sportifs... c'est une association, moi, qui vraiment me porte » (Sébastien, AG, membre). Relevons enfin que les camps de ski pour enfants transplantés s'inscrivent à la jonction de l'entre-soi et du soutien psychosocial d'un côté, et de l'affirmation des vertus de l'activité physique sportive de l'autre côté. L'objectif est en effet de donner l'occasion à ces enfants (et leurs familles) de nouer des liens d'entraide, et surtout de leur permettre de développer leur personnalité et de se prouver qu'ils sont des enfants comme les autres. L'organisatrice de ces activités souligne le bénéfice psychologique sur les participants; "les enfants qui viennent chez nous sont traités par leurs parents comme s'ils étaient faits en verre, ils sont couvés, ils ont peur... et quand ils partent de chez nous, ils ont changé, ils repartent plein de vie, ils ont plein de projets [...] la différence que nous faisons à la vie de ces jeunes compte beaucoup pour moi » (Nadia, FD, comité).

Si elle peut offrir des avantages similaires à ceux d'autres activités associatives (vie sociale, partage d'expériences, de conseils ou d'informations pratiques), pour certains membres, la spécificité du sport, de surcroît de compétition, réside dans le fait qu'il représente 
un puissant marqueur de normalité et de qualité de vie. En outre, le statut de sportif constitue le point de référence identitaire de nombreux responsables et membres associatifs, au-delà de celui de transplanté ${ }^{600}$. Nous montrons plus loin que la mise à distance de l'image du «transplanté malade» vise aussi à modifier la représentation sociale du transplanté.

\section{L'aide concrète}

Le soutien des associations prend aussi la forme d'une aide concrète et pratique. Il peut s'agir d'une aide administrative, consistant à fournir des informations pour guider les patients dans leurs démarches avec les assurances maladie notamment, et pour les orienter vers les services adéquats, qu'il s'agisse de services sociaux ou d'adresses de professionnels de santé, comme des diététiciens. Des coups de main pour chercher un appartement par exemple ou d'autres petits services ponctuels sont aussi évoqués par certains acteurs associatifs. L'aide concrète revêt aussi une forme matérielle, à l'instar des associations qui possèdent des véhicules destinés à transporter les malades dans les centres de dialyse ou des transplantés lors de leurs visites médicales dans les centres hospitaliers éloignés. Il s'agit aussi de mettre à disposition des appartements pour accueillir les familles de patients venant de subir une transplantation ou de louer un chalet à la montagne pour les membres et leurs proches. Dans la mesure de leurs moyens, certaines associations proposent une aide financière, en lien notamment avec des frais de santé qui ne seraient pas pris en charge par l'assurance maladie, ou pour payer les dialyses lors de vacances à l'étranger, sous forme de prêt ou de don. L'aide financière, rappelons-le, est en partie à l'origine de la création de plusieurs associations: "Les insuffisants rénaux devaient faire des dialyses, et quand ils allaient à l'étranger, les caisses maladies ne payaient pas d'avance, elles ne prenaient pas

600 L'importance de la dimension identitaire pour la mobilisation collective en général a été soulignée dans la littérature: "la capacité d'un groupe à se doter d'une identité forte et valorisante - fût-elle imaginée - constitue une ressource de première importance pour que ses membres intériorisent une vision de leur potentiel d'action [et] que le collectif s'affirme dans l'espace public [...]" (Neveu 2015a, 80). 
en charge, donc il y a un fonds qui a été constitué pour aider et faire des avances aux gens qui prenaient des vacances en faisant des dialyses, au départ, c'était un aspect important» (Jean, AR, comité).

Certaines associations transmettent également des informations utiles à leurs membres par le biais d'un journal interne, d'un rapport d'activités étoffé ou de l'organisation de conférences ${ }^{601}$. Elles concernent par exemple les statistiques ou les aspects légaux du don d'organes, le droit du travail ou de l'assurance invalidité. Mais ce sont les problèmes de santé et les aspects médicaux liés à la transplantation qui sont le plus souvent abordés: outre des conseils de santé, des informations relatives aux médicaments, aux traitements immunosuppresseurs, à une maladie particulière ou encore au suivi médical ${ }^{602}$. L'éducation thérapeutique à proprement parler constitue cependant une activité très marginale des associations.

Sans exclure pour autant des actions d'information et de promotion du don d'organes, l'entraide au sens large constitue la mission principale de plusieurs groupements. Tel président qualifie son association de "caritative » et revendique pleinement, comme d'autres, le caractère local (c'est-à-dire cantonal) de son engagement: «ce à quoi elle sert, c'est de donner des petits coups de pouce à gauche, à droite [...] notre association, on l'a faite autour de l'aide chez nous, pour les personnes d'ici» (Pierre, AR, comité). Un autre président souligne: «Notre but [est] d'aider nos membres quand ils ont des problèmes, dans des moments de dépression, de les soutenir moralement, et puis dans la mesure du possible financièrement" (Thomas, AR, comité). L'importance du soutien large aux patients concerne aussi certaines fondations: "Cette fondation n'est pas faite que pour la

601 C'est le cas notamment de l'AST qui met sur pied chaque année depuis 2003 (initialement sous l'égide des As de Cœur), en collaboration avec Swisstransplant et plusieurs associations de patients, et avec le soutien d'entreprises pharmaceutiques, un "Symposium pour transplantés", dans lequel des aspects médicaux, psychologiques, sociaux ou juridiques de la transplantation sont abordés par des spécialistes.

602 Le Bulletin de l'AST et le Journal de la SSPIR donnent un bon aperçu des thèmes traités en lien avec la transplantation: la sexualité, la grossesse, l'alimentation, le sommeil, le sport, les voyages, la dialyse, les médicaments génériques, l'hygiène bucco-dentaire, la biopsie du muscle cardiaque, l'acide mycophénolique ou encore la médecine régénérative. 
sensibilisation de la communication mais surtout pour apporter un soutien sur le plan social, administratif» (Murielle, FD, comité).

Si les activités orientées vers les besoins des membres sont très diverses, elles ont pour point commun de chercher à faciliter le vécu de la condition de transplantés ou de candidats à une transplantation, en répondant à leurs difficultés, qu'elles soient sociales, psychologiques, économiques, administratives ou matérielles. Le retour à une qualité de vie quasi normale après une greffe constitue en effet un défi non négligeable de réintégration socioprofessionnelle et d'adaptation psychologique au nouvel organe ${ }^{603}$. Ces différents types d'aides font très largement écho à la fonction traditionnelle et historique des associations de soutien aux malades, en termes de socialisation et de «réaffiliation sociale ${ }^{604}$ », de partage d'expériences, de transmission d'informations et de savoirs, permettant aux patients de se prendre en charge (self help) et d'intégrer leur identité de malades ${ }^{605}$.

\section{Informer et sensibiliser le public}

L'information du public sur le don d'organes en tant que pratique sociale et problème de santé publique constitue un deuxième objectif majeur, affiché par toutes les associations (voir chapitre 6). Certains responsables soulignent que la promotion du don d'organes représente la principale raison d'être de leur collectif, revendiquant parfois «une différence de vocation » vis-à-vis des associations œuvrant dans l'aide et le soutien aux membres; "nous on n'est pas une association de greffés, on est une association qui promeut le don d'organes» (Bernard, AG, comité). La responsable d'un autre collectif insiste sur la différence entre ses activités tournées vers la cause du don d'organes et les activités de soutien aux patients; "[notre groupement] est pour la promotion du don d'organes et l'information, ce n'est pas pour rencontrer des gens, etc., pour faire un club de transplantés... pas du tout» (Adeline, AG, comité). Dans cette

603 Engle 2001, Neukom et al. 2012, Schulz et Kroencke 2015, Youngner 2003.

604 Ngatcha-Ribert 2012, 296.

605 Herzlich et Pierret 1991, Rabeharisoa et Callon 2000a. 
optique, les associations organisent ou participent à des événements très divers afin de rendre la question du don d'organes davantage visible dans l'espace public et présente dans les consciences individuelles. Sans prétendre à l'exhaustivité, nous mentionnons les activités et stratégies les plus significatives en lien avec cet objectif ${ }^{606}$.

À des fréquences variables, quasiment toutes les associations tiennent des stands d'information dans la rue, notamment lors de la Journée nationale du don d'organes qui mobilise chaque année depuis 1994 différents acteurs du don d'organes qui se coordonnent pour assurer une visibilité dans de nombreuses villes. Des manifestations ponctuelles sont aussi l'occasion pour certaines associations de tenir un stand, telles que des événements sportifs (meetings d'athlétisme, courses à pied, concours hippiques...), des braderies, des marchés ou des concerts. Des stands sont également tenus en dehors de manifestation particulière dans des centres commerciaux ou sur des places publiques. Plusieurs associations mettent également sur pied des événements spécifiques de grande envergure et au fort potentiel médiatique. Ces événements ont le plus souvent un caractère sportif: tournoi de golf, match de football, régate, course à pied ou à vélo, et compétitions de ski notamment, à l’instar des jeux mondiaux d'hiver des transplantés. Dans un autre registre, on peut mentionner la publication d'ouvrages relatant l'expérience d'un proche ou d'une personne transplantée, ainsi qu'un film sur le don d'organes réalisé par Tackers, traduit dans plusieurs langues et diffusé dans des classes d'école de divers pays. Quelques associations se sont également investies dans des activités artistiques pour faire parler du don d'organes (concerts de musique, pièce de théâtre, concours littéraire ou de dessin, édition d'un recueil de poèmes...), ainsi que dans des événements à caractère symbolique pour témoigner de la gratitude des receveurs vis-à-vis des donneurs et leurs familles (plaque commémorative, arbre du souvenir...).

606 Nombre d'événements et manifestations organisés par les associations impliquent la collaboration active d'autres acteurs, en particulier Swisstransplant et des hôpitaux, mais aussi d'autres associations de patients, les pouvoirs publics ou des partenaires privés, pharmaceutiques par exemple. Par souci de concision, nous ne précisons pas les partenaires et les soutiens des événements mentionnés dans cette section. 
Enfin, la plupart des associations sont actives dans l'organisation de conférences ou soirées d'information, généralement en collaboration avec un médecin ou une infirmière impliqués dans la transplantation. Si ces interventions publiques sont destinées à la population tout-venant, elles visent particulièrement les jeunes, jugés davantage "réceptifs", par le biais d'exposés dans le cadre scolaire (lycées, collèges et écoles professionnelles) ou lors d'écoles de recrues. À travers ces divers événements, les associations cherchent certes à accroître la visibilité sociale du thème du don d'organes, mais aussi à favoriser leur propre visibilité auprès de la population et de membres potentiels.

\section{Les messages à faire passer}

Prioritaire ou secondaire, l'objectif d'information et de sensibilisation du public constitue un horizon commun de toutes les associations. Cependant, ces notions sont rarement discutées ou problématisées en tant que telles dans les discours des interviewés. Les termes «information", "promotion" ou «sensibilisation" ne font guère l'objet d'une conceptualisation et sont souvent utilisés de manière équivalente par les acteurs associatifs. Néanmoins, l'analyse des entretiens fait ressortir plusieurs modalités des messages qu'ils cherchent à faire passer dans la population.

L'objectif des stands d'information et des conférences publiques consiste à faciliter le dialogue sur le don d'organes, considéré comme mal connu et trop peu visible. Pour une part, les échanges avec la population visent à transmettre des faits sur l'activité de transplantation et le don d'organes, et à mieux faire connaître un domaine de la médecine souvent chargé de représentations erronées et suscitant des craintes et des réticences, comme nous l'avons souligné au chapitre précédent. L'effort d'information du public revêt alors une dimension didactique; "c'est cela que l'on essayait d'inculquer aux gens: les connaissances médicales, leur expliquer comment on fait une opération du cœur, une opération du foie, des reins, une opération du pancréas, etc. donc on essayait d'expliquer» (Claude, AG, comité). Il s'agit aussi d'apporter des réponses relatives au cadre 
éthico-légal et aux conditions médicales du prélèvement (critères pour être donneur, anonymat, gratuité, respect du corps...). Dans le registre des peurs et des "fantasmes", la nécessité de "rassurer» le public est omniprésente, notamment par rapport au diagnostic de la mort cérébrale qui, aux dires de nombreux interviewés, suscite des doutes; «beaucoup de gens ne savent pas qu'un moment donné, ce sont des neurologues qui viennent et qui ne travaillent pas pour l'unité de transplantation, ça, c'est quand même fondamental, alors ça évidemment il faut l'expliquer aux gens »(Daniel, AG, comité). Les rencontres et échanges directs avec la population visent aussi à souligner l'importance du don d'organes et de la problématique de la pénurie, ainsi que ses conséquences pour les malades (nombre de décès sur liste d'attente, durée de l'attente pour recevoir un nouvel organe...). Plusieurs arguments déployés reflètent ceux des milieux officiels de la transplantation, à l'instar du risque épidémiologique: «l'argument principal c'est quand même de dire que vous avez, entre guillemets, plus de chances d'avoir besoin d'un organe que d'en donner un" (Laurent, FD, membre). Il s'agit donc de faire passer le message que tout un chacun est concerné par l'éventualité de recourir à une transplantation, à titre personnel ou pour un proche.

La diffusion de la carte de donneur constitue un autre aspect important des activités associatives. Le nombre de cartes distribuées est souvent brandi comme la marque du succès de leur rôle; "je pense que dans le [canton], on a fait une bonne propagande, pendant en tout cas six, sept ans, je suis sûr que l'on a le $60 \%$ des personnes qui ont leur carte de donneur» (Pierre, AR, comité). La promotion de la carte de donneur rejoint l'objectif des instances officielles et des professionnels de la transplantation: favoriser l'expression de l'autodétermination de chacun et éviter le poids d'une décision difficile à ses proches dans une situation de don potentiel.

Enfin, la qualité de vie retrouvée grâce à la technique de la transplantation constitue l'un des messages déterminants à faire passer auprès du public; "de les sensibiliser sur le fait qu'on peut quand même vivre avec... ça permet quand même à des gens de vivre mieux, comme moi disons... simplement avec un don qui ne 
coûte pas grand-chose» (Laurent, FD, membre ${ }^{607}$. Tel est également l'objectif du film produit par l'une des associations: "si on peut dire: "On est encore là, on a fait ça, ça et ça, tout va bien, voilà mes petits-enfants" [montrer que la vie continue] oui, c'est un thème que j'utilise souvent quand je fais des présentations " (Nadia, FD, comité). Le sport, nous y revenons ci-après, et plus encore les exploits sportifs permettent de montrer l'efficacité de la transplantation au public, comme le relatent fréquemment les bulletins d'associations $^{608}$. La «facilité » et la beauté du geste du don sont aussi des éléments soulignés dans les discussions avec la population. Le message que le don d'organes sauve des vies ${ }^{609}$ et que chacun est un bienfaiteur potentiel est central: "Je dis qu'on a la possibilité chacun sans être médecin de sauver la vie de plusieurs personnes si quelque chose nous arrive. C’est magnifique...»(Nadia, FD, comité).

Pour résumer, les visées principales des acteurs associatifs en matière d'information sont les suivantes: faire connaître le don d'organes dans l'espace public et rendre la question plus visible socialement, augmenter le niveau de connaissances de la population sur la transplantation, rassurer et rectifier certaines croyances, faire prendre conscience de la pénurie d'organes comme problème de santé publique, montrer l'efficacité et la noblesse du geste, ou encore inciter tout un chacun à la réflexion. S'inscrivant globalement

607 Montrer la valeur thérapeutique de la transplantation et la qualité de vie qui en découle n'est pas un argument propre aux associations, mais un élément central de l'ensemble des discours publics de sensibilisation sur le don d'organes. Par exemple, la manière dont la Transplant Cup de 2008, sous forme de régate, est mise en avant dans le bulletin de Swisstransplant s'inscrit dans cette logique: "Cet événement sportif a de nouveau été en mesure de sensibiliser un large public au don d'organes et de proposer aux participants une manifestation sportive pleine de joie de vivre, de plaisir et d'espoir de voir "en vrai" les succès de la transplantation grâce au don d'organes" (Swisstransplant News, septembre 2008, 13).

608 "L'édition 2007 du Marathon de la Jungfrau, qui se courra le 8 septembre l'année prochaine, sera l'occasion d'attirer l'attention du public sur le don d'organes, en montrant que le don d'organes, loin d'assurer la simple survie d'un malade, lui offre un nouveau départ avec une qualité de vie excellente qui rend possibles des performances extraordinaires" (Bulletin de l'AST, n² 26, août 2006, 12).

609 Omniprésent au niveau international dans les discours institutionnels et les campagnes de promotion, le message du don de vie (gift of life) est au cœur de la construction culturelle contemporaine du don d'organes, comme nous l'avons évoqué dans l'Introduction (Healy 2006, Moloney et Walker 2002). 
dans les fonctions attribuées à l'information (voir chapitre 8), ces messages couvrent les trois orientations généralement associées aux campagnes de santé publique: agir sur les connaissances de la population, sur les attitudes et dispositions des individus, et enfin sur leurs comportements ou pratiques ${ }^{610}$. Rappelons, à la suite des chapitres précédents, que certaines stratégies concrètes d'encouragement au don d'organes (la rhétorique du besoin personnel ou de la souffrance des malades en attente...) ne font pas nécessairement consensus parmi les acteurs associatifs.

\section{Un travail de proximité indispensable}

Si à bien des égards leurs messages sont similaires aux discours des professionnels de la transplantation, la spécificité des associations réside dans leur travail de proximité auprès du public. Elles sont nombreuses en effet à souligner l'importance du dialogue direct avec les citoyens comme une dimension clé de leurs activités; "chez nous, c'est quand même le travail de proximité qui compte, ça j’en suis persuadée... on essaie de persévérer là-dedans, on est à l'écoute... chaque fois que l'on fait des informations, il y a beaucoup de monde qui vient... je pense que c'est dans ce sens que l'on arrivera à leur faire comprendre que c'est comme toute maladie, cela peut arriver à tout le monde»(Brigitte, AR, comité). Être au contact des gens, à l'écoute de leurs questions, pouvoir répondre à leurs préoccupations, aller à la rencontre des plus jeunes lors d'exposés dans le cadre scolaire, privilégier les échanges interpersonnels... autant d'activités qui apparaissent comme une compétence propre au milieu associatif. Cette proximité avec le public est d'autant plus nécessaire que de nombreux interviewés la conçoivent comme étant à même de surmonter les réticences de la population et d'encourager les donneurs potentiels. Le travail d'information réalisé sur le terrain et les discussions individuelles sont jugés comme des moyens essentiels de sensibilisation à la cause du don d'organes; "en amont, les associations peuvent faire du travail positif aussi, parce que le don d'organes, on l'explique sur les stands... c'est très peu connu,

610 Logan 2010. 
c'est une chose qu'il faut expliquer, il faut parler aux gens, parce que sans dialogue c'est cuit! »(Claude, AG, comité). Plusieurs interviewés livrent diverses anecdotes montrant que la communication personnalisée et directe a permis de convaincre certains citoyens du bien-fondé du don d'organes; "Je sais déjà que des gens ont été donneurs grâce à certaines actions avec qui j'avais affaire, ça c'est énorme pour moi » (Nadia, FD, comité).

En somme, si l'information du public doit être améliorée, les associations, grâce au contact direct avec la population, revendiquent la capacité de faire avancer la cause du don d'organes; "l'information, c'est la meilleure chose, mais cela doit se faire dans les associations, au niveau cantonal, ce n'est pas au niveau suisse» (Brigitte, AR, comité). Si ces propos dénotent la légitimité de l'implication locale de plusieurs associations et surtout le peu de crédit et de pertinence accordés aux campagnes d'information (voir chapitre 7), la valorisation du travail de proximité se cristallise aussi fortement dans le pouvoir du témoignage, au croisement des opérations d'information et de sensibilisation (voir chapitre 8).

\section{Témoigner pour la cause:}

authenticité et normalisation de la figure du transplanté

Le témoignage des transplantés représente un élément central du travail de proximité des associations et de légitimation de leur rôle. Que ce soit à l'occasion d'exposés dans des écoles, de conférences publiques, d'entretiens dans les médias ou de discussions dans la rue, la plupart des interviewés évoquent dans des termes similaires l'importance de parler de leur expérience personnelle.

Les vertus attribuées au témoignage résident dans le fait de (dé)montrer l'issue concrète d'un don d'organes en même temps que le bienfait médical d'une transplantation. À contre-courant des stéréotypes d'une personne diminuée ou recluse, la valeur du témoignage s'inscrit aussi dans la modification de l'image sociale d'un transplanté en mettant en avant la normalité (retrouvée) grâce à un nouvel organe; "ce sont des gens qui dans la vie font du sport, ils travaillent $[. .$.$] ce sont vraiment des gens normaux, je pense que c'est$ 
cela qu'il faut vraiment communiquer, c'est la qualité de vie qu'on reçoit, c'est la vie que l'on reçoit» (Gilles, AG, comité). L'exemple concret et "vivant» de la réussite de la transplantation représente en tant que tel un argument de promotion du don d'organes; "cela leur fait voir que cela marche, que l'on n'est pas une moitié de légume, il y en a beaucoup qui pensent que d'être transplanté, c'est survivre encore et que l'on n'a pas un niveau de vie excellent" (Stéphanie, AR, comité).

Comme on l'a noté précédemment, la pratique sportive ou l'activité physique constitue fréquemment un marqueur public de normalité611. À l'exemple des jeux mondiaux d'hiver des transplantés, les compétitions sportives contribuent à façonner socialement la figure du transplanté non comme une personne malade, invalide, diminuée ou encore sédentaire, mais comme quelqu'un qui a retrouvé ses capacités fonctionnelles; "ça, c'est un bon modèle de dire: "Je peux faire des courses de ski [...] bien que je sois transplanté”... grâce à la transplantation on peut dire: "Ça, je peux faire" [...] le fait de voir que des gens qui ont été transplantés peuvent bouger, vivre comme tout le monde, même faire un petit peu plus, ça c'est important » (Léo, AG, membre). Pour cette responsable d'une association qui organise des événements à caractère sportif, la valorisation de la figure du transplanté s'inscrit dans une stratégie délibérée de communication de l'efficacité de la greffe et de promotion du don; «on a mis en avant les transplantés pour montrer que ces gens grâce à un don, ils vivent comme vous et moi [...] le but de tout ça [...] c'est pour sensibiliser le public à la réflexion » (Murielle, FD, comité). Si la pratique du sport peut constituer un critère personnel de normalité de la vie après une transplantation, elle permet aussi de façonner une certaine image publique du transplanté qui revêt un enjeu

611 Outre au sport et à l'activité professionnelle, le thème de la normalité est aussi adossé à des activités quotidiennes et banales, comme l'illustre ce compte rendu d'une activité de l'AST; " pour la sortie à l'est, nous étions accompagnés d'un journaliste et d'un photographe, qui voulaient faire un reportage sur des transplantés. C'est avec surprise qu'ils ont constaté que nous ressemblions à "un groupe normal de randonneurs". Oui, il est merveilleux pour nous de pouvoir vivre "normalement" grâce à un don d'organe. Faire des excursions, découvrir des nouveautés, rencontrer des amis, danser [...]" (Bulletin de l'AST, $\mathrm{n}^{\circ}$ 44, novembre 2014, 15). 
important pour les acteurs associatifs. Le témoignage individuel (médiatique ou non) représente ainsi une ressource de légitimation du don d'organes comme pratique médicale et de normalisation sociale du transplanté lui-même ${ }^{612}$.

Si l'expérience personnelle peut en soi avoir valeur de promotion, ce sont les émotions du témoignage qui donnent toute sa signification et sa force à l'opération de sensibilisation: "rendre concret" et "faire agir ${ }^{613}$ ". Les émotions sont perçues comme le vecteur privilégié d'une prise de conscience morale de l'importance du don d'organes. Les parcours dramatiques des personnes greffées se prêtent particulièrement bien à l'exploitation des sensibilités. Ce transplanté cardiaque évoque ainsi les éléments enclins à susciter l'empathie et l'admiration de l'auditoire lors de ses interventions publiques: l'imminence de la mort et la figure du rescapé, la souffrance, la lutte pour la (sur)vie, l'abnégation, l'incertitude, le destin heureux. "Je cherche pas bien loin, je prends mon exemple, ce qui s'est passé, je devais partir bien avant... le début de la liste d'attente, parce que j'ai fait soixante-trois jours de coma, et puis soixante-huit jours de soins intensifs d'une traite... il paraît qu'ils ne misaient pas 5 centimes sur moi, mais bon ils ne m'ont pas eu... [...] c'est après que j'explique vraiment que j'avais pas envie de crever [...] je veux faire comprendre aux gens qu'il arrive un certain moment où il faut prendre une décision, mais il faut réfléchir, il faut écouter le pour et le contre, alors moi, c'est clair, je donne mon pour avec le récit» (Bernard, AG, comité). L'instrumentalisation du registre pathémique est ici parfaitement assumée; "c'est par le récit de où j’ai passé [...] les gens sortent le Kleenex et machin [...] il faut être franc, cela ne sert à rien de tourner autour du pot, il faut vraiment crocher les gens

612 Les progrès médicaux de la transplantation sont des facteurs déterminants qui conditionnent en grande partie ce phénomène de normalisation, à l'exemple de l'hémophilie (Carricaburu 1993). Dans son volet individuel, la normalisation du statut de transplanté implique, comme pour nombre de maladies chroniques (Bury 1991), d'intégrer dans la vie quotidienne les traitements et ses éventuels effets secondaires ainsi que ses contraintes et précautions du point de vue de l'hygiène de vie, mais aussi d'intégrer le nouvel organe dans son schéma corporel, de se réadapter dans la vie sociale, familiale et professionnelle, ou encore d'identifier ses nouvelles capacités et limites physiques. 
dans l'émotif, si vous les touchez pas avec l'émotif, cela ne va pas toucher [...] et puis là les gens sur [le coup de] l'émotion se disent: "Ouais, c'est vrai on ne peut pas laisser tomber", et c'est vrai qu'on n'a pas le droit de laisser crever les gens comme ça... » (Bernard, AG, comité). L'expérience personnelle du don d'organes peut ainsi faire l'objet d'un «travail émotionnel » au sens où les émotions mobilisées dans le témoignage constituent un répertoire d'actions ${ }^{614}$, y compris dans la presse grand public ${ }^{615}$. Selon Neveu, «le privilège donné au ressort émotionnel est la marque d'entrepreneurs de cause peu professionnels, de séquences émergentes de problèmes ${ }^{616}$ ».

Pour beaucoup d'interviewés, la force de la parole du patient tient dans l'authenticité et la singularité de l'expérience de celui ou celle qui est directement concerné dans sa chair : le corps abîmé, souffrant et réparé incarne la vérité des enjeux du don d'organes, comme geste moral et ressource inestimable de vie, mais aussi comme problème de santé publique en termes de pénurie. À travers le témoignage, les acteurs associatifs revendiquent ainsi une expertise expérientielle ${ }^{617}$ et une légitimité spécifique pour parler du don d'organes, qui sont autant de ressources politiques. Dans un courrier adressé à Swisstransplant en 2004, la présidente d'une association avançait précisément l'argument de l'authenticité pour renforcer leur collaboration: "Ces différentes associations et fondations, nées d'un peu partout, forment un atout majeur pour Swisstransplant puisqu'elles symbolisent, par des groupes d'individus volontairement constitués,

614 Latté 2015.

615 Amey et Hammer 2010. Le recours au registre émotionnel pour favoriser l'adhésion du public au don d'organes est l'un des éléments constitutifs des discours institutionnels et associatifs sur le sujet. Virginie Plumart relate qu'au début des années 1980, "le président national [de France-Transplant] encourageait [...] les bénévoles à se situer sur le registre de l'émulation des affects afin d'obtenir l'adhésion de la population: il fallait "émouvoir, exciter la compassion des gens", faire mention de "la situation pénible des malades", de leurs "attentes angoissées", et ne pas oublier de dire que des enfants avaient également besoin de greffes" $(2012,103)$.

616 Neveu 2015b, 140-141.

617 À la suite des travaux de Borkman, Akrich et Rabeharisoa proposent la définition suivante: "l'expertise expérientielle [...] se traduit par une compétence à mobiliser le savoir expérientiel pour apporter des réponses à des problèmes formulés en termes généraux ou spécifiques" (2012, 70). 
l'authenticité du problème dans le quotidien » (Adeline, AG, comité). Cette spécificité du milieu associatif est d'ailleurs reconnue par des acteurs institutionnels du don d'organes. Dans un courrier daté de 2002, Trix Heberlein, alors présidente de Swisstransplant, voyait d'un bon œil les initiatives profanes en faveur du don d'organes, en l'occurrence la création alors récente de la fondation Passezle-Relais; "pour nous, cet engagement pour la promotion du don d'organes est très important et comme il s'agit des personnes qui ont vécu des moments douloureux et décisifs, ces expériences sont plus crédibles que les appels de notre organisation ». Dans ce contexte, l'expertise des personnes transplantées tend à s'inscrire davantage dans un rapport de complémentarité, voire d'alliance avec les professionnels de la santé et les organismes du don d'organes, que dans un rapport de dénonciation ou d'opposition frontale ${ }^{618}$.

\section{Les médias}

Outre la couverture médiatique des événements d'information et de promotion du don d'organes (Journée nationale du don d'organes, manifestations sportives, conférences publiques...), l'intervention directe dans les médias est l'un des moyens de parler de la problématique du don d'organes et de son expérience personnelle de transplanté ${ }^{619}$. Pour autant, si certaines associations n'ont guère d'écho dans les journaux, hormis de façon ponctuelle dans des quotidiens locaux, d'autres ont pu jouir d'une couverture médiatique régulière de leurs activités (conférences de presse, interviews à la radio, dans la presse, et plus rarement à la télévision). Bénéficier de relais médiatiques constitue ainsi une stratégie complémentaire à la présence sur le terrain comme moyen de sensibiliser le public. Pour autant, le rapport aux médias est souvent marqué du sceau de l'ambivalence, caractérisé par la nécessité de jouer le jeu pour

618 Lochard et Simonet 2009.

619 Mentionnons aussi, moins fréquents, les événements mondains (soirées de gala, concerts...) qu'organisent plusieurs associations dont l'objectif est multiple: faire connaître l'association et ses buts grâce au concours de célébrités et personnalités qui favorise l'attention médiatique; faire parler du don d'organes; et récolter des fonds pour financer ses activités de sensibilisation à la cause. 
exister, en se pliant aux règles de la logique médiatique. Si la presse généraliste notamment traite du sujet du don d'organes, c'est souvent moins dans une optique purement promotionnelle qu'en raison de son caractère sortant de l'ordinaire. Cet interviewé souligne ainsi que l'intérêt médiatique est conditionné à un contenu accrocheur pour les lecteurs: "Les médias, on les croche avec quelque chose de spécial, parce qu'autrement ils entendent pas, il y a des séries où les journaux ont bien parlé du don d'organes, et puis maintenant, c'est silence radio depuis un moment» (Bernard, AG, comité). D’autres relèvent combien la couverture médiatique obéit à l'agenda institutionnel; "Il faut qu'il y ait des événements pour qu'ils en parlent, alors évidemment quand il y a la Journée du don d'organes, eh bien, ils en parlent un petit peu, ils en remettent une couche et puis voilà » (Léo, AG, membre).

Illustrant un rapport instrumental aux médias, Carine indique qu'il faut savoir "appâter " les médias avec de «l'émotionnel »: «Pendant deux ans, j'ai beaucoup utilisé des patients greffés où ils racontaient toujours les mêmes histoires, ça s'effrite au bout d'un moment... c'est comme si vous mettez un produit sur le marché et que vous avez la même étagère et l'étagère devient usée à force de servir le même produit, il faut changer» (FD, comité). Se soumettant aussi à la logique médiatique en proposant du spectaculaire, de l'inédit ou la présence de célébrités, Nadia (FD, comité) décrivait ce jeu comme un mal nécessaire pour avoir une certaine visibilité. Enfin, les propos de ce président résument le lien de dépendance et l'ambivalence des associations vis-à-vis du pouvoir des médias et de la recherche du sensationnalisme: "On a la stratégie du pauvre, comme on n'a pas de sous, on espère que les journalistes vont nous faire un bon papier [...] mais ils font un papier qui les arrange! Mais qui n'arrange pas forcément la cause, et on voit [que] tous ces articles qui sortent sont des ramassis de contre-vérités parfois, qui font froid dans le dos ${ }^{620} »$ (Marc, FD, comité). Que les interviewés témoignent

620 Le rapport tendu qu'expriment plusieurs acteurs associatifs vis-à-vis des médias n'est pas propre à la question du don d'organes. La critique du sensationnalisme et de la déformation des faits scientifiques de la presse s'observe par exemple dans les associations en lien avec le sida (Barbot 1999) et l'hémophilie (Carricaburu 1993). 
de leur satisfaction ou au contraire de leur mécontentement à l'égard du traitement médiatique du don d'organes, ils ont bien conscience du rôle majeur que peuvent jouer les médias dans la signification du don d'organes dans l'espace public.

\section{Agir sur le politique: un objectif marginal}

Alors que l'activisme associatif dans la sphère politique constitue l'une des caractéristiques de la mobilisation collective des patients au cours des dernières décennies ${ }^{\mathbf{6 2 1}}$, force est de constater qu'il en va bien différemment dans le domaine de la transplantation d'organes. Les actions visant à agir directement au niveau des instances politiques, cantonales ou fédérales, sont en effet minoritaires. L'engagement politique, cherchant par exemple à modifier le cadre institutionnel de la régulation du don d'organes, les modalités de l'action publique ou à défendre explicitement les intérêts des malades, constitue ainsi une ambition marginale au sein de l'espace associatif.

\section{L'engagement politique de Passez-le-Relais}

La fondation Passez-le-Relais est la seule association dont l'engagement était fortement axé sur le registre politique. Son objectif, rappelons-le, consistait à modifier la régulation du don d'organes en créant un registre officieux de donneurs et non-donneurs à l'échelle nationale. Aux dires de la fondatrice et présidente, plus de 50000 personnes ont été inscrites à son registre. L'introduction au début des années 2000 de ce moyen d'expression de la volonté des citoyens constituait une alternative concurrente au système en vigueur de la carte de donneur, soutenu par les milieux de la transplantation, Swisstransplant notamment. Par la suite, Passezle-Relais changea d'objectif et eut l'ambition de faire figurer le statut de donneur ou non-donneur sur un document officiel ${ }^{622}$, tel que la carte d'assurance maladie que tout résident suisse possède. Les propositions défendues par la fondation visaient à simplifier

621 Carricaburu et Ménoret 2004, Dodier 2003.

622 Cette question a été discutée à plusieurs reprises au Parlement au cours des dernières années. 
en amont l'organisation de la transplantation, notamment pour les professionnels, mais traduisaient aussi un acte symbolique en faveur du don d'organes en lui donnant une reconnaissance politique et une visibilité sociale accrues. En particulier, la mention du statut de donneur sur un document officiel signifierait que la disposition vis-à-vis du don représente une question existentielle et sanitaire de première importance, justifiant l'obligation ou l'incitation forte à ce que chaque citoyen prenne position.

Durant près d'une dizaine d'années, la responsable de Passez-leRelais, entourée de quelques membres et bénévoles, a multiplié les actions afin de rendre visible la cause du don d'organes et de tenter de convaincre le monde politique de ses projets. Outre les événements mondains impliquant des célébrités sportives et des hommes politiques, destinés à récolter des fonds pour financer ses activités et à se faire connaître du grand public, son engagement principal était dirigé vers les plus hautes sphères de l'État: "Si on ne change pas le politique [...], si on n'a pas le levier du pouvoir, on n'obtiendra jamais rien.» C'est ainsi qu'elle a mené un lobbying politique soutenu dans les couloirs du Parlement fédéral à Berne: "J'ai fait deux ans de salle des pas perdus à toucher des mains de gauche et de droite. » La proposition de créer un registre national de donneurs a ainsi été débattue au Parlement fédéral mais sans succès ${ }^{623}$. Les projets de la fondation ont été loin de faire l'objet d'un consensus dans les milieux politiques et de la transplantation. Sa responsable indique que son combat s'est pendant plusieurs années heurté à de vives oppositions, notamment de la part de Swisstransplant ${ }^{624}$.

623 Une telle proposition a fait l'objet d'une motion au Conseil national fin 2013, rejetée par le Conseil fédéral. Fin 2018, Swisstransplant a mis sur pied un registre national du don d'organes, base de données électronique permettant d'enregistrer sa décision personnelle pour ou contre le prélèvement de ses organes et tissus à des fins de transplantation.

624 Ces oppositions sont aussi dues aux circonstances particulières de l'engagement de la fondatrice - l'un de ses fils fut donneur de plusieurs organes. Suite à un concours de circonstances, elle a noué des liens avec la mère du receveur du cœur de son fils, receveur qui devint par la suite un membre actif de la fondation. Ce faisant, la responsable de Passez-le-Relais transgressait l'anonymat, principe majeur du don d'organes. Ses visions sur la manière d'organiser le don d'organes en Suisse étaient "contradictoires" avec les règles en vigueur, pour reprendre les termes d'une personne responsable de Swisstransplant. Celle-ci nous a également confirmé que les milieux 
De manière très ponctuelle et moins engagée, d'autres associations ont été impliquées dans des actions de nature politique, à l'exemple de prises de position auprès du Parlement fédéral pour la défense des intérêts des transplantés dans le cadre de la sixième révision de l'assurance invalidité. De même, l'AST est intervenue brièvement en 2013 auprès de la Commission de la sécurité sociale et de la santé publique du Conseil des États pour «défendre [...] la cause du don d'organes au niveau politique ${ }^{625} »$. En outre, quelques associations ont été entendues dans les phases préparatoires de la LTx pour défendre le consentement présumé ${ }^{626}$. Enfin, des représentants des patients greffés ont pu s'exprimer dans le cadre de la révision partielle de la LTx. Au-delà de ces quelques exemples et à l'exception de Passez-le-Relais, les associations en lien avec le don d'organes ont été peu investies dans des actions concrètes, a fortiori contestataires et publiques, visant à peser directement sur les politiques sanitaires ${ }^{627}$.

\section{D'une mise à distance à de nouvelles ambitions politiques?}

La définition des enjeux d'une mobilisation collective n'est pas indépendante du choix des stratégies et des modes d'action ${ }^{628}$. La marginalité de l'engagement politique des associations résulte en

médicaux n'ont jamais attesté que le transplanté en question avait effectivement reçu le cœur du fils.

625 Bulletin de l'AST, n 43, mai 2014. Dans un autre registre, on peut également mentionner la lutte menée par une association locale pour empêcher la fermeture d'une antenne de dialyse.

626 Selon un médecin impliqué dans ce processus politique, les associations avançaient en ordre dispersé et avaient des prises de position "un peu différentes les unes des autres, avec des nuances dans les messages [...], si les associations avaient été unies, peut-être que cela aurait eu un impact [sur la loi]" (entretien). Voir aussi Chenaux (2000).

627 La préoccupation politique n'est pas pour autant absente des discours associatifs. Ainsi, "un plus grand poids politique" est présenté comme l'un des avantages de la fusion en 2005 entre Les As de Cœur et Trans-Hepar (Les As de Cœur News, $n^{\circ} 19$, avril 2004, 6). Le franchissement du nombre de 400 membres au sein de l'AST constitue "un énorme succès qui accroît notre influence face aux médias et aux politiques" (AST News, $n^{\circ}$ 41, mai 2013, 1).

628 Barbot 1999. 
partie de leurs objectifs: la centration sur l'entraide ou le soutien matériel aux malades, et le choix de privilégier un travail de sensibilisation à la cause du don par des activités de communication directement auprès de la population. Pourtant, nous l'avons vu, la plupart des interviewés interprètent volontiers, davantage à titre individuel que comme représentant associatif, le don d'organes comme un problème en grande partie politique, tant au niveau des causes que des moyens d'y remédier (voir chapitre 7). L'implantation locale des activités de la plupart des associations tient aussi aux faibles ressources humaines et financières dont elles disposent (nous y revenons plus loin). Aux dires de plusieurs responsables de «petites» associations, l'action au niveau politique est hors de portée, leur priorité étant de remplir au mieux les missions qu'elles se sont fixées avec les moyens du bord. Il convient de noter aussi chez quelques interviewés un désintérêt assumé vis-à-vis des aspects politico-législatifs du don d'organes et un refus explicite de s'engager sur le terrain politique. Par exemple, tout en reconnaissant qu' «il y a beaucoup à faire au niveau politique ", cette responsable cible son engagement sur le terrain concret: "Tout ce que je veux faire, c'est dire combien je suis heureuse, dire merci, montrer ce que nos donneurs ont fait [...], faire des activités... Je suis égoïste, moi je ne veux pas aller faire du lobbying» (Nadia, FD, comité). On observe ainsi globalement dans l'espace associatif une mise à distance du politique, délibérée ou circonstancielle, tant dans les objectifs que dans le type d'action.

Néanmoins, après de nombreuses années d'implication associative, quelques interviewés envisagent de réorienter leurs objectifs vers la sphère politique, estimant que c'est la seule voie d'un changement significatif: "Nous sommes maintenant dans un carrefour de notre partenariat, et c'est sûr que nous allons augmenter notre pression sur Swisstransplant et sur l'OFSP, avec un certain nombre de projets [qui] sont en élaboration... Nous allons porter sur la place fédérale la problématique» (Daniel, AG, comité). C'est également le cas de ce président qui souhaite agir au niveau des instances nationales afin que la loi permette à l'État de promouvoir le don d'organes et d'introduire le consentement présumé: «Une des zones que j’aime- 
rais privilégier maintenant, c'est le travail avec les politiques, parce que c'est un travail de base, et il faut que l'on puisse avoir un réel impact auprès des politiques, pour leur dire, attention, attendez, ce qu'il y a aujourd'hui, ce n'est pas viable» (Marc, FD, comité). Un premier pas dans cette direction a consisté dans le lancement d'une pétition sur Internet en faveur de la révision de la LTx et du passage au consentement présumé ${ }^{629}$. Cette volonté de réorienter la stratégie d'action découle d'un changement de perception du problème: la sensibilisation du public ne peut à elle seule améliorer la situation. «La population, elle est acquise, maintenant ce qui n'est pas acquis, c'est les représentants de la population, donc c'est là-dessus qu'il faut travailler... [...] je me suis rendu compte [que] s'il n'y avait pas les infrastructures derrière qui fonctionnaient et qui entérinaient cette position favorable de la population, on n'arriverait à rien " (Marc, FD, comité). Le président d'une grande association projette également de s'engager davantage au niveau politique et souhaite "organiser un apéritif avec les gens qui sont dans le Parlement, pendant les sessions, pour leur parler du don d'organes, pour leur parler de ce changement [...] il faut faire quelque chose» (Gilles, AG, comité). Cet intérêt pour le politique transparaît également chez d'autres interviewés, à l'instar de Sébastien (AG, membre) qui, tout en étant très satisfait des activités de son association, regrette qu'elle ne cherche pas davantage à promouvoir le don d'organes dans la société - il verrait d'un bon œil qu'elle cherche à avoir un "poids politique » et qu'elle exerce des «pressions».

\subsection{Rôle et pouvoir des associations}

Après avoir décrit les principales stratégies et activités des associations, il faut s'interroger sur leur capacité d'action. À cet effet, nous examinons d'abord le bilan que tirent les responsables associatifs pour nous intéresser ensuite aux obstacles et limites du pouvoir de mobilisation des associations, tant du point de vue de

629 Cette pétition était sans lien avec l'initiative populaire lancée en automne 2017 par la Jeune chambre internationale de la Riviera en faveur du consentement présumé. 
leurs caractéristiques internes que des changements récents dans le contexte de régulation du don d'organes en Suisse.

\section{À l'heure du bilan: succès et regrets}

À l'heure du bilan, les interviewés brossent un tableau contrasté de l'impact des activités menées et de la situation de leur association. Au rang des aspects jugés le plus positivement figurent les activités liées à l'entraide et au soutien psychosocial. Plusieurs responsables estiment que leur association répond aux attentes et besoins des patients en leur fournissant une aide adaptée et en proposant des activités conviviales qui ont favorisé les liens entre les membres. Un président se félicite d'avoir pu «rendre l'association plus vivante vis-à-vis de nos membres, de leur montrer qu'on est là, qu'on est une association" (Thomas, AR, comité). Un autre souligne «les relations créées avec les membres, qui sont très positives" (Jean, AR, comité). Quant à cette responsable de l'organisation de camps de sports d'hiver, elle relève la satisfaction de voir au fil des années les enfants transplantés devenir des adultes épanouis et en bonne santé (Nadia, FD, comité). Si les activités d'entraide et de sociabilité entre pairs sont globalement perçues comme des succès, y compris par certains membres, quelques interviewés estiment néanmoins que leur association devrait faire davantage pour le soutien psychosocial ou la (ré)insertion professionnelle des personnes transplantées.

Les activités d'information et de sensibilisation du public suscitent des appréciations positives plus mesurées, dès lors qu'elles sont contrebalancées par le regard critique porté sur la situation globale de la transplantation. Alors que les acteurs associatifs estiment avoir contribué à mieux faire connaître le don d'organes grâce à diverses actions auprès du public, nombreux sont ceux qui relèvent aussi les limites de l'exercice. Ce responsable juge que le sujet «don d'organes » a gagné en visibilité sociale mais souligne qu'il reste beaucoup à faire sur le plan de l'information et des représentations individuelles: "Nous nous rendons compte que dans ces stands, de moins en moins de gens tombent de la lune en entendant parler du don d'organes, de la transplantation [...] par contre, si le sujet 
lui-même n'est plus à ce point étranger ou étrange, il demeure beaucoup de fantasmes, de craintes, de peurs»(Daniel, AG, comité). Un autre se dit satisfait d'avoir pu favoriser l'image positive de la transplantation grâce aux contacts avec la population tout en restant très prudent à propos de l'efficacité des actions entreprises: "C'était un peu une goutte d'eau et puis je me dis: même si à la limite il y avait deux personnes sensibles, eh bien voilà, c'est toujours deux personnes de plus» (Laurent, FD, membre).

Dans un autre registre, si Marc loue le succès de plusieurs manifestations publiques importantes qu'il a mises sur pied pour promouvoir le don d'organes - «On a quand même réussi à initier une dynamique, il y a des personnes qui ont été touchées, et qui conservent un souvenir extraordinaire »-, c'est un sentiment en demi-teinte qui prédomine: "Ce qui a été fait, cela a été bien fait, ça a eu un impact limité certes $[. .$.$] ça a plus [...] enfoncé des portes ouvertes, c'est-à-dire continué à$ convaincre des gens qui étaient déjà presque convaincus et c'est certainement utile de faire cela » (FD, comité). D’autres acteurs associatifs, tout en étant plutôt satisfaits de la publicité faite au don d'organes, soulignent les difficultés d'engager un véritable échange avec le public sur le sujet. Un autre bémol souvent exprimé concerne le manque de visibilité des associations elles-mêmes dans l'espace public, qu'il s'agisse de ne pas avoir pu ou voulu se faire mieux connaître auprès de la population et des médias ${ }^{630}$, et de ne pas avoir davantage cherché d'appuis du côté du monde politique.

Compte tenu des énergies déployées et des moyens limités à disposition, les actions de sensibilisation auprès de la population sont généralement considérées comme des succès d'estime. L'état des lieux est cependant terni par le sentiment que la cause du don d'organes demeure peu soutenue par les pouvoirs publics (voir

\footnotetext{
630 Dans quelques cas, le manque de visibilité concerne aussi les patients eux-mêmes et les professionnels de la santé, comme l'illustre une association cantonale en lien avec le rein: "La première chose à faire est de se faire connaître. La triste vérité est que très peu de monde (patients et/ou médecins/personnel soignant) connaît notre existence. Ce constat est bien affligeant et il faut y remédier au plus vite [...]. Nous devons collaborer avec les néphrologues et le personnel soignant pour qu'ils relaient l'information aux patients, pour que ceux-ci sachent que nous existons et que nous pouvons peut-être les soutenir, répondre à leurs questions" (Journal de la SSPIR, $n^{\circ} 1,2012$ ).
} 
chapitre 7). L'impression de buter contre l'inertie institutionnelle, la frilosité politique, les tabous culturels et l'indifférence d'une partie de la société a tendance à contrebalancer l'évaluation positive des efforts accomplis. Par exemple, si Carine est satisfaite d'avoir contribué à donner «une autre image [du don d'organes]" dans la population et d'avoir "fait quand même bouger beaucoup de choses ", elle relève qu'elle s'est heurtée au système: "Je ne peux pas faire changer le monde politique, et c'est pas mon rôle» (FD, comité). Le bilan ambivalent des activités d'information du public et de la promotion de la cause tient ainsi à la confrontation des deux niveaux d'appréciation de la situation du don d'organes. À l'échelle de l'association et de ses moyens limités, le bilan est favorable, mais à l'échelle du contexte sociopolitique helvétique, l'impact des activités associatives paraît généralement faible ou dérisoire. Laurent résume parfaitement ce sentiment mitigé: "Le côté associatif c'est vraiment des petites gouttes d'eau comme ça... mais bon plusieurs gouttes d'eau, ça fait quelque chose... mais quand même... disons au niveau suisse, il y a une grosse machine à bouger, c'est un peu compliqué » (FD, membre). Si le bilan contrasté des associations renvoie à la difficulté de faire bouger les choses politiquement et dans les mentalités, il tient aussi aux obstacles rencontrés dans la réalisation de leurs activités.

\section{Obstacles et limites de la mobilisation}

Qu'il s'agisse de la capacité d'agir sur le monde extérieur ou de répondre aux besoins des membres, le pouvoir de mobilisation de toute association dépend de ses ressources et de son mode de fonctionnement, mais aussi du contexte dans lequel elle se situe ${ }^{631}$. Son devenir, le succès ou l'échec de ses activités sont influencés par de multiples paramètres tant internes qu'externes ${ }^{\mathbf{6 3 2}}$. L'objectif n'est pas ici de passer en revue de manière systématique les facteurs pouvant

\footnotetext{
631 Pinell 2007.
}

632 La diversité des théories de l'action collective et de l'engagement en sociologie et en sciences politiques reflète cette complexité dans l'appréhension de la mobilisation associative et du pouvoir des collectifs citoyens (voir par exemple Neveu 2015a, Fillieule et Péchu 1993, Cefaï 2007). 
rendre compte de la mobilisation des associations autour du don d'organes, mais de mettre en avant les éléments les plus significatifs de leur dynamique.

\section{Ressources et logique d'engagement}

Les ressources internes des associations constituent un enjeu crucial. Comme indiqué précédemment, leurs moyens financiers sont généralement faibles, composés principalement des cotisations des membres, de donations privées ou de revenus liés aux activités associatives. Les subventions publiques, lorsqu'elles existent, sont modestes et ponctuelles. Loin d'être couronnée de succès, la quête de subventions mobilise beaucoup d'énergie et engendre une situation de concurrence entre les associations puisqu'elles sont parfois amenées à solliciter les mêmes sources possibles de financement.

Les ressources humaines représentent un autre problème majeur pour la plupart des associations. Rappelons d'abord que plusieurs d'entre elles ont peu de membres. Ensuite, le recrutement laborieux de nouveaux membres prêts à s'engager, jeunes en particulier, constitue une doléance récurrente, quand ce n'est pas le constat d'une baisse des membres ${ }^{633}$. L'apport de «sang neuf» et le renouvellement des fonctions dirigeantes sont alors compromis. Dans ces conditions, l'écueil le plus significatif réside dans le fait que la «vitalité » de certaines associations repose sur l'implication active d'un petit nombre d'individus, qui peut être d'un ou deux. L'effectif réduit des personnes qui «portent» l'association constitue un obstacle à la continuité d'autant plus sérieux, si ce n'est insurmontable, que leur santé est rendue fragile par leur parcours médical et leur âge. Le dynamisme et parfois la survie de plusieurs, notamment petites, associations sont ainsi étroitement liés à un noyau d'individus particulièrement engagés. Le sort de l'association se confond alors avec le parcours de son fondateur ou de son président en place de longue date. Une

\footnotetext{
633 Le phénomène n'est pas nouveau. Par exemple, en 2003, le président de Trans-Hepar lançait un appel pour recruter des bénévoles romands: "Con questo appello voglio tentare per l'ultima volta di reclutare due nuovi membri per la Svizzera romanda attorno ai quali dovrebbe ruotare l'attività regionale della nostra associazione" (Les As de Cœur News, $n^{\circ} 17$, août 2003, 14).
} 
démission, un décès, la péjoration de l'état de santé ou l'usure de l'engagement d'une personne peut sonner le glas de l'association. Des tensions internes peuvent aussi contribuer à la vulnérabilité d'un collectif, a fortiori s'il est constitué de quelques membres.

La dépendance de certains groupements à l'implication d'un nombre restreint de personnes s'explique en partie par les conditions de l'engagement initial pour le don d'organes. Il n'est pas rare en effet que des patients transplantés décident de créer une association, ou de s'y investir, dans une logique apparentée à un contre-don. S'engager dans un collectif pour promouvoir la cause est une manière pour certains de "rendre» ce qu'ils ont reçu. La personnalisation du combat pour le don d'organes peut ainsi entrầner une personnalisation du collectif qui obère sa pérennité et la continuité des activités par-delà l'engagement individuel. Cette logique de l'implication associative n'est certes pas généralisable à toutes les associations enquêtées, mais elle est significative dans plusieurs cas. Si cet aspect constitue probablement l'une des particularités de la thématique du don d'organes, il s'ajoute au manque de moyens financiers, au petit nombre de membres actifs et à la faible professionnalisation du fonctionnement associatif, qui représentent à l'évidence des obstacles qui pèsent sur le potentiel d'action.

\section{La capacité à se faire entendre}

La capacité des associations du don d'organes à se faire entendre dans la société dépend aussi de leur insertion dans le champ de la transplantation et dans le système social plus largement. À cet égard, le pouvoir limité des associations dans l'espace public et dans les décisions politiques tient notamment au fait que peu d'entre elles peuvent compter sur des appuis auprès des instances politiques et administratives (aux niveaux local et fédéral), et au fait que celles qui jouissent de relais médiatiques sont l'exception plutôt que la règle. Il faut relever également que les liens avec Swisstransplant et le monde médical varient considérablement d'une association à l'autre. Certaines, à l'instar de Tackers ou de l'AST, ont développé des liens importants de coopération avec Swisstransplant - «On peut parler 
d'un vrai partenariat maintenant", indique un haut responsable de Swisstransplant. D'autres associations ont des liens de proximité avec le monde hospitalier grâce à la profession de santé d'un des responsables ou à la présence de médecins ou d'infirmières dans le comité de telle association ou fondation. À l'inverse, quelques acteurs associatifs expriment des relations plutôt conflictuelles et des critiques à l'égard de Swisstransplant, jugeant la collaboration ou le dialogue difficile ${ }^{634}$. Plusieurs d'entre eux nuancent toutefois leur propos en soulignant l'amélioration récente des relations avec le changement intervenu à la présidence de la fondation nationale. Si certains acteurs associatifs regrettent que les relations avec Swisstransplant ne soient pas plus fructueuses, d'autres ne cherchent pas particulièrement à développer des liens avec cet organisme, préférant mener leurs activités de manière indépendante. S'il est difficile d'évaluer dans quelle mesure la nature des relations avec Swisstransplant influe sur la capacité d'action et la visibilité des associations, il ressort que la plupart d'entre elles se trouvent dans une situation "segmentée ${ }^{635}$ ", c'est-à-dire à distance des acteurs significatifs de la transplantation, politiques et médicaux notamment.

L'espace associatif du don d'organes se caractérise également par une coopération limitée entre les associations, pour des raisons aussi diverses que la poursuite d'objectifs différents, une personnalisation de la cause, des zones d'influence bien définies, des inimitiés personnelles, voire des luttes de territoire et des enjeux de pouvoir et d'image ${ }^{636}$. Si l'histoire de l'espace associatif n'est pas exempte de

634 Ces rapports tendus renvoient généralement à des événements anciens et sont de plusieurs ordres: des querelles personnelles, des conflits d'ordre financier et de transparence comptable, mais aussi des conflits liés à l'utilisation du nom ou du logo de Swisstransplant, ou encore à la manière d'aborder le public - "une communication parfois trop agressive pour le don d'organes", selon une source interne à Swisstransplant.

635 Ce terme, emprunté à Oberschall, s'oppose à la situation d'un groupe "intégré" qui "dispose de connexions stables lui donnant des chances d'être entendu des autorités supérieures" (Neveu 2015a, 53).

636 Une personne responsable au sein de Swisstransplant nous décrivait la situation du début des années 2000 , marquée par des "conflits d'intérêts, des conflits personnels" entre associations; "tout le monde se battait pour la même cause, mais il y avait peu, voire pas de communication entre elles... c'était toujours un peu cette peur de se faire prendre la clientèle, si on allait au marché à [nom de localité], il fallait être sûr qu'il n'y avait pas une autre association qui [y] était déjà stationnée." 
fusions (voir chapitre 6), elle est aussi marquée par les velléités de plusieurs groupements de travailler ensemble ou d'unir leurs forces, sans réel succès, ainsi que par des ruptures. Par exemple, suite à des conflits, l'une des associations de patients en lien avec le rein a quitté l'association faîtière (SSPIR). Certaines activités (tenue de stands, manifestations sportives, conférences, soirées mondaines...) réunissent de façon ponctuelle les efforts et les ressources de plusieurs associations dans un climat de bonne entente ${ }^{637}$. Mais c'est globalement une situation de fragmentation de la mobilisation collective qui prévaut, dans un contexte caractérisé par des ressources financières restreintes, un renouvellement difficile des engagements individuels, et des changements institutionnels dans la régulation du don d'organes, sur lesquels nous revenons plus loin. Commentant la première Journée mondiale pour le don et la transplantation d'organes tenue à Genève en 2005, le vice-président de France ADOT (Fédération des associations pour le don d'organes et de tissus humains) relevait «l'individualisme des associations» en Suisse, en référence aux « 48 associations [qui] œuvrent en faveur du don d'organes ${ }^{\mathbf{6 3 8}} »$. Cette situation de «turfism» ${ }^{\mathbf{6 3 9}}$ - terme désignant un défaut de coalitions entre associations autour d'un objet commun constitue un obstacle à la construction efficace d'une cause ${ }^{640}$ et à une visibilité forte dans l'espace public. Dans le domaine du sida ${ }^{641}$ ou de la maladie d'Alzheimer ${ }^{642}$, par exemple, la création d'alliances, au-delà des divisions, a favorisé la capacité des associations à se faire entendre des pouvoirs publics.

637 Depuis le milieu des années 2000, Swisstransplant invite une fois par an les responsables des différentes associations de Suisse à se réunir dans ses locaux. Aux dires des responsables de Swisstransplant, ces rencontres ont permis d'améliorer les relations entre et avec les associations.

638 Source: www.france-adot.org/images/pj/78p1_Premiere\%20Journee\%20 Mondiale\%20-\%20Site.pdf

639 Wood 2000.

640 Bach 1995.

641 Barbot 2002, Voegtli et Fillieule 2012.

642 Ngatcha-Ribert 2012. 
Pour comprendre la marginalité de l'action politique et l'influence limitée des associations sur les politiques publiques, les caractéristiques sociales des acteurs associatifs entrent également en ligne de compte. Les circonstances de l'engagement dans un collectif de santé sont généralement liées à la survenue d'une maladie - que l'on soit touché à titre personnel ou à travers un proche. La proximité avec la maladie est donc un élément déterminant de l'implication des individus $^{643}$, à la différence des motivations qui ont donné lieu aux «nouveaux mouvements sociaux ${ }^{644}$ ». L'engagement profane pour le don d'organes est, au départ, moins de nature «idéologique » qu' "accidentelle », c'est-à-dire l'expérience d'une lourde épreuve de santé dans laquelle la vie est souvent en jeu. Cela peut expliquer que les acteurs associatifs enquêtés, à quelques exceptions près, sont des primo-militants, qui n'ont donc ni un parcours ni une âme de militant. Rarement dotés de compétences préalables en matière d'action collective et de lutte face aux pouvoirs publics, ils ne sont pas forcément familiers des rouages de la politique institutionnelle ${ }^{645}$ et n'inscrivent pas la question du don d'organes dans un combat plus large ${ }^{646}$. Outre la valorisation de l'entraide, l'absence de passé

643 Knobé 2009. Précisons que le devoir de charité à l'égard des personnes vulnérables peut aussi être une motivation à l'engagement associatif, dans l'esprit philanthropique qui a structuré les premières mobilisations contre des maladies à la fin du XIX ${ }^{\ominus}$ et au début du $X X^{\ominus}$ siècle.

644 Neveu 2015 a.

645 Comme relevé plus haut, certains responsables associatifs ne s'intéressent pas au politique ou connaissent mal les développements politico-législatifs récents du don d'organes en Suisse.

646 Le contexte et les facteurs de la mobilisation profane autour du don d'organes sont très éloignés de ceux de la mobilisation associative exceptionnelle contre le sida au début de l'épidémie (Pinell 2002). Barbot mentionne ainsi "les principaux facteurs qui ont favorisé la mobilisation des homosexuels: la disposition d'un capital culturel, l'appartenance aux classes moyennes supérieures et la possibilité d'y mobiliser des alliés, la préexistence de réseaux de sociabilité volontaires, de liens électifs entre des individus, constitués ou renforcés par la conscience d'être socialement menacés" (1999, 158159). L'exception notable à ce tableau d'ensemble est la fondatrice de Passez-le-Relais - une des rares responsables d'association non transplantée - dont l'activisme sur le terrain politique et médiatique doit beaucoup aux compétences acquises durant sa formation: "Je me suis mise en route comme si j'allais prendre un job [...] apprendre un vocabulaire différent, utiliser mes clés marketing-communication de ma formation de base pour pouvoir approcher les gens." 
militant peut expliquer le rapport distancié au politique, comme cela a été également observé dans la lutte contre le cancer du sein ${ }^{647}$.

Enfin, la culture politique helvétique peut aussi expliquer l'intérêt limité des milieux associatifs pour défendre une cause dans un rapport de force avec l'État. Plusieurs travaux suggèrent en effet que l'expression citoyenne privilégie les canaux institutionnels de la démocratie directe plutôt que des moyens de confrontation ${ }^{648}$. Comparativement à d'autres pays, les mobilisations collectives en Suisse recourent moins à des actions spectaculaires et violentes, ou à des stratégies de protestation publique telles que les grèves ou les manifestations de rue.

\section{Changements institutionnels et rôle des associations}

À l'instar de la lutte contre le sida en Suisse ${ }^{649}$, la dynamique de l'espace associatif n'est pas indépendante des changements institutionnels, en particulier l'évolution de la régulation de la transplantation. Incontestablement, l'entrée en vigueur de la LTx le $1^{\text {er }}$ juillet 2007 correspond à une nouvelle donne politique dans le domaine du don d'organes. Rappelons que jusqu'à cette date la médecine de transplantation était régulée par un arrêté fédéral, des arrêts du Tribunal fédéral et par des réglementations cantonales hétérogènes, notamment par rapport au type de consentement. Le don d'organes comme question de santé publique était alors une affaire gérée essentiellement à l'échelle des cantons, en l'absence de politique nationale ${ }^{650}$. Les campagnes d'information sur le don d'organes étaient de la compétence des autorités sanitaires cantonales et n'étaient guère fréquentes. Merminod rappelle qu'avant 2007 «la Confédération n'avait jamais réalisé de campagnes à propos du don d'organes et de la médecine de transplantation du fait de l'inexistence d'un mandat

647 Knobé 2011.

648 Balsiger 2016.

649 Voegtli et Fillieule 2012.

650 Sur le plan de l'attribution des organes ou des échanges entre centres hospitaliers, des accords existaient entre ces derniers, notamment sous les auspices de Swisstransplant et de l'ASSM (voir chapitre 3). 
législatif correspondant ${ }^{651}$ ». Swisstransplant organisait divers événements d'information et de sensibilisation du public, à l'instar de la Journée nationale du don d'organes (voir chapitre 2), mais ces opérations étaient modestes compte tenu du budget "quasi inexistant » pour la communication, selon l'un des responsables de la fondation.

Dans ce contexte, les associations ont assurément joué un rôle actif et pionnier dans la communication publique sur le don d'organes - plusieurs associations ont été fondées bien avant la création de Swisstransplant en 1985. On relèvera notamment que l'une des premières cartes de donneur, si ce n'est la première en Suisse, a été créée par l'association Les As de Cœur en $1987^{652}$. Jusqu'au début des années 2000, les associations ont assumé une part importante de l'information sur le don d'organes et de sa promotion auprès de la population. Dans un contexte de vacance politique relative (l'État fédéral est en retrait et le don d'organes n'est pas véritablement constitué en problème public), la place des associations est plus facilement acquise: "Lorsque le terrain est dégagé, délaissé par les grands acteurs institutionnels de la santé, les associations de malades correspondantes sont en mesure de jouer un rôle irremplaçable, la légitimité de leur intervention étant assurée ${ }^{653}$.»

La LTx modifie ce contexte en profondeur avec deux conséquences importantes au moins pour les associations. Premièrement, on assiste à une politisation accrue du don d'organes, de la pénurie et de la médecine de transplantation puisque ces questions relèvent désormais de la compétence d'instances fédérales et de politiques publiques à l'échelle nationale. Cette "mise en politique $^{654}$ » signifie aussi l'entrée en scène et l'intervention de nouveaux acteurs, en particulier l'OFSP qui reçoit pour mission, sur mandat de la Confédération, d'informer la population, alors que

651 Merminod 2013, 135.

652 À ce moment, deux cartes sont mises en circulation: l'une directement accessible produite par Les As de Cœur, l'autre de Swisstransplant que le public pouvait obtenir après avoir rempli un formulaire de demande adressé à la fondation créée alors peu de temps auparavant.

653 Rabeharisoa et Callon 2000b, 1226.

654 Barthe 2006. 
Swisstransplant hérite de la promotion du don d'organes. Avec la LTx, Swisstransplant a pu renforcer son assise grâce à l'obtention de moyens financiers accrus, notamment pour les relations publiques. Par rapport à la période qui précède 2007, la régulation change d'échelle avec les nouvelles prérogatives dont est doté l'État, qui de fait devient le «propriétaire ${ }^{655}$ » du don d'organes comme problème public. Compte tenu de cette redistribution des compétences et des ressources, les associations perdent de leur influence dans la «capacité à créer ou orienter la définition publique d'un problème ${ }^{656}$ ». Ajoutons que la politisation équivaut aussi à une bureaucratisation et à une autonomisation de la gestion des questions relatives au don d'organes.

Deuxièmement, la LTx implique l'émergence d'un cadrage officiel de la question du don d'organes, chargé d'orientations symboliques et politiques fortes. Si le législateur a sans surprise entériné les principes éthiques de la gratuité, de l'anonymat et d'un acte librement consenti, les choix du consentement explicite élargi et de la neutralité de l'État ont suscité des critiques de la part de plusieurs professionnels de la transplantation, d'acteurs associatifs et de juristes ${ }^{657}$. Rappelons que dans le cadre de la LTx le rôle de l'État n'est pas d'inciter ou non les citoyens à devenir donneurs, mais de les informer et de les encourager à prendre position. Il s'agit « d'offrir au public les conditions-cadres pour choisir l'option qui lui semble préférable, à savoir donner ou ne pas donner ses organes ${ }^{658}$ ». En regard des attentes d'une partie des acteurs concernés par la transplantation $^{659}$, la législation suisse traduit une reconnaissance symbolique faible de l'enjeu du don d'organes. L'accent officiellement mis sur l'information et la neutralité plutôt que sur la promotion ne reflète

655 Gusfield 2009.

656 Gusfield 2009, 11.

657 Mader 2010.

658 Merminod 2013, 136.

659 Au début des années 2000, la perspective d'une loi nationale sur la transplantation était envisagée comme un moyen d'harmoniser les pratiques en Suisse, mais aussi comme une opportunité de généraliser la règle du consentement présumé - considérée comme l'une des mesures à même d'atténuer le manque d'organes. 
dans l'ensemble pas les objectifs des associations et la manière dont leurs responsables conçoivent le problème. On l'a noté précédemment, les acteurs associatifs plaident pour que les pouvoirs publics fassent davantage pour le don d'organes: "[ils] attendent plus de visibilité, plus d'engagement vers un oui, tout aussi bien de l'Office fédéral de la santé publique que de la part de Swisstransplant ", comme l'indique une personne responsable de Swisstransplant. Sans pour autant être nécessairement en porte-à-faux, la perception du problème par les acteurs associatifs n'est pas (ou plus) dans la même lignée que la définition officiellement défendue par le gouvernement.

À titre d'exemple, la question des causes de la pénurie d'organes s'est sensiblement modifiée, passant d'un cadrage axé sur le manque de donneurs à un cadrage mettant en exergue le manque de dons, pour reprendre la distinction de Prottas et Batten ${ }^{660}$. En effet, durant plusieurs années, si l'on en juge par les discours dans la presse des associations, patients et professionnels de la transplantation, la pénurie était surtout interprétée sous l'angle d'une disposition individuelle au don défaillante, renvoyant à un problème d'ordre culturel, cognitif ou émotionnel ${ }^{661}$. Progressivement, l'organisation et les pratiques hospitalières, incluant l'identification des donneurs potentiels, ont pris davantage de place dans les discours des professionnels, des acteurs associatifs et dans les mesures politiques ${ }^{662}$. Ainsi, parmi les mesures du plan d'action national «Plus d'organes pour des transplantations", trois concernent des aspects médicohospitaliers ("formation du personnel médical ", "processus et

660 Prottas et Batten 1989.

661 Hammer 2012.

662 Ce changement de cadrage peut être illustré par l'attention portée à un article de la Weltwoche dans un journal associatif, commenté en ces termes: "Ce retard [manque de greffons] a depuis des années été imputé, apparemment à tort, aux faibles taux de consentement. En effet, selon l'article [...] le problème de l'insuffisance des prélèvements d'organes s'explique essentiellement par le manque de formation du personnel soignant et par les restrictions budgétaires des hôpitaux régionaux" (Les As de Cœur News, novembre 2003, $n^{\circ} 18,6$ ). Les propos du Pr Philippe Morel, l'un des pontes de la transplantation en Suisse, témoignent également de ce changement de perception; "[...] le problème n'est plus seulement lié à l'information "grand public", mais également à un contrôle intra-hospitalier qui doit être effectué régulièrement, obligatoirement, et dans l'ensemble des hôpitaux ayant des soins intensifs" (Les As de Cœur News, décembre 2004, $\mathrm{n}^{\circ} 21,14$ ). 
gestion de la qualité », et "structures et ressources des hôpitaux") et une l'information dans la population ("campagnes et relations publiques»). Ce déplacement rend moins consensuelle ou évidente la définition du problème de la pénurie et du type de solutions. En outre, les associations ont a priori peu d'influence sur la question de l'optimisation des pratiques hospitalières ou des stratégies médicales pour pallier la pénurie d'organes.

Conjuguées aux obstacles et limites mentionnés plus haut, la politisation et la redéfinition partielle des contours du problème public du don d'organes depuis un peu plus d'une dizaine d'années peuvent expliquer les limites de la mobilisation des associations. Avec l'introduction de la LTx, le vide politique et symbolique que compensaient en partie les associations a été comblé par l'État. Le renforcement de l'intervention des pouvoirs publics et le changement d'échelle de la régulation du don d'organes soulèvent la question de la mission des associations aujourd'hui. Il est intéressant de relever à cet égard qu'aux yeux des acteurs associatifs la LTx n'a pas eu d'incidence majeure sur leur rôle - rares sont ceux pour qui la division du travail issue de la LTx remet en question la mission de promotion du don d'organes du secteur associatif. Cela reflète probablement la nature des activités associatives (essentiellement tournées vers les membres - soutien et aide - et la population - information et sensibilisation), en même temps que la grille de lecture peu politique de la mobilisation collective des patients. 



\section{Conclusion de la Partie II}

\section{Un espace associatif en recomposition}

\section{Raphaël Hammer}

À l'issue de notre enquête en 2015, l'espace associatif romand du don d'organes apparait comme fragile et marqué par un affaiblissement ou un essoufflement de la mobilisation. Ce constat est aussi celui de certains acteurs associatifs. Ainsi, à l'occasion de la Journée mondiale du rein en mars 2011 à Berne, «la SSPIR a été déçue du peu de ses membres ayant répondu à l'appel de cette action qui fut pourtant réussie ${ }^{663}[\ldots]$.. Des observateurs privilégiés livrent une appréciation similaire, comme ce haut responsable de Swisstransplant: «Ce qu'on observe, nous, c'est qu'ils sont de moins en moins actifs dans des stands, ils se retirent, il y a un certain manque de gens motivés, la volonté de sortir pour promouvoir le sujet a baissé. » Ce déclin de la vitalité de l'espace associatif au cours des dernières années indique que les années 1990-2000 constituent probablement l'âge d'or de la mobilisation associative en lien avec le don d'organes en Suisse romande. À cet égard, notre enquête a permis de documenter sociologiquement la fin de cette période marquée par une nouvelle donne politique du don d'organes, et apporte des éléments de réflexion sur la recomposition actuelle de l'espace associatif.

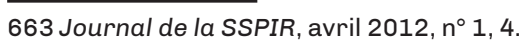


S'il faut relever la dissolution en 2014 de la fondation Passezle-Relais, la plupart des associations se situent à un tournant de leur histoire. Deux situations peuvent être distinguées. La première reflète une phase de transition (ou reconstruction), dans laquelle se trouvent deux fondations et, à une exception près, toutes les associations en lien avec l'insuffisance et la transplantation rénales. Les responsables de ces groupements font état d'une stagnation ou d'une suspension des activités qu'ils expliquent par la nécessité de redéfinir leurs objectifs et actions, et de recruter de nouvelles forces vives. La seconde situation peut être qualifiée de critique dès lors que la pérennité des activités de trois associations est incertaine, si ce n'est celle des associations elles-mêmes ${ }^{664}$. Le président et cofondateur de l'une d'elles a démissionné et renoncé à son engagement pour des raisons de santé après plusieurs années de forte implication. Cette démission a constitué un coup dur pour cette petite association généraliste comme l'indiquait son vice-président et cofondateur, soulignant également la difficulté de lui trouver un successeur. Une autre association généraliste se trouve dans un cas de figure similaire en raison du décès de sa fondatrice en 2014, qui occupait alors la fonction de vice-présidente après plusieurs années de présidence. Il s'agit enfin d'une association en lien avec le rein dont le président et l'un des membres s'interrogeaient sur la pertinence de l'existence même de l'association au vu de la diminution de la participation et d'un désintérêt accru des membres. Par contraste avec ces situations d'incertitude, une fondation et une grande association généraliste s'inscrivent dans une trajectoire de continuité. Aux dires de leurs responsables, elles ne sont pas confrontées à des difficultés particulières du point de vue des ressources leur permettant de mener à bien leurs activités, et n'envisagent pas de nouvelles stratégies ou de redéfinir le sens de leurs actions.

À l'exception de cette minorité d'associations caractérisées par la stabilité et le dynamisme de leurs activités, force est de constater que l'espace associatif romand du don d'organes est en phase de

664 En suivant Morin (1976), la situation de crise ne signifie pas nécessairement que l'association est en situation de disparition mais qu'elle se situe à un moment décisif de son histoire, qui peut par exemple évoluer vers une transformation profonde. 
recomposition. Il est donc légitime de s'interroger sur le futur des associations. Connaîtront-elles un regain de leurs activités actuelles dans la continuité de leurs objectifs initiaux? Sont-elles au contraire destinées à repenser leurs stratégies et s'engager dans de nouvelles actions? Leur viabilité dépend-elle seulement d'un « rajeunissement » de leurs comités et d'une «modernisation» de leurs modes de fonctionnement? L'espace associatif est-il au contraire condamné à une disparition progressive et à renaître sous une forme totalement renouvelée, impliquant une nouvelle génération de groupements et de militants? Plus fondamentalement se pose la question du rôle social et politique des associations du don d'organes.

Seul l'avenir apportera bien sûr des réponses à ces questions, mais quelques pistes peuvent être esquissées. En matière de stratégie de mobilisation publique, deux grandes options se présentent. Compte tenu des discours critiques à l'égard du manque d'engagement de l'État, une première option consisterait à se mobiliser contre les pouvoirs publics en faveur de l'introduction du consentement présumé et de l'abandon de la neutralité ${ }^{665}$. Une stratégie de confrontation ou de dénonciation publique impliquerait des modes d'action et un profil sociologique de militants inédits dans le cas du don d'organes. De plus, une position d'extériorité et de contestation est difficile à maintenir dans la durée et requiert des relais efficaces, à l'instar des médias, et des ressources permettant de se faire entendre et de faire pression. Enfin, compte tenu de la sensibilité morale et symbolique de l'enjeu du don d'organes, il convient de ne pas sous-estimer le poids des contraintes discursives sur la prise de parole publique. Au sens de Fassin ${ }^{666}$, l'économie morale de la transplantation implique en effet des valeurs, des normes et des émotions qui balisent ce qui

665 Un tel changement politique est possible, compte tenu du succès de l'initiative de la Jeune chambre internationale de la Riviera en faveur du passage à un système de consentement présumé (voir la partie Enjeux actuels et perspectives). Cette initiative fait suite au rejet de l'introduction du modèle du consentement présumé par le Parlement, notamment dans le cadre de la révision de la LTx en 2015. En 2012, à une large majorité de ses membres, la Commission nationale d'éthique pour la médecine humaine s'était prononcée contre le consentement présumé, arguant que celui-ci irait à l'encontre des droits de la personnalité. 
est dicible ou non, en termes de justification ou de contestation du don d'organes. Pour rester crédible politiquement et audible socialement, la mobilisation autour du don d'organes ne peut pas produire n'importe quel argument ou recourir à n'importe quelle action. Par exemple, les principes du respect du corps et du défunt apparaissent comme intangibles, de même que les principes de la gratuité et du don librement consenti. En outre, la promotion du don devient disqualifiante si elle est perçue comme trop insistante, le déni de la souffrance des personnes en attente de transplantation est difficilement recevable, au même titre que la stigmatisation des non-donneurs.

Néanmoins, on peut avancer que c'est précisément le rôle d'une association d'assumer la position de contradicteur des pouvoirs publics, profitant de leur liberté de parole et de ton, ne serait-ce que pour (ré)activer le débat social ou politique. On peut concevoir que la fonction des associations consiste à nourrir la pluralité des opinions en défendant vigoureusement la promotion du don d'organes. C'est en substance le propos d'une personne responsable au sein de Swisstransplant: «Est-ce que ce n'est pas justement aux associations de jouer le diable et de dire "il faut y aller, il faut dire oui" [...] est-ce que ce n'est pas finalement leur travail parce qu'eux n'ont pas les menottes qu'on a nous [référence au cadre législatif]...»

Selon Neveu: "Le complément le plus efficace au processus de mobilisation reste de s'introduire durablement dans les négociations avec les autorités, de pénétrer dans le cercle des agents habilités à gérer un type de dossier ${ }^{667}$. » La stratégie d'intégration - seconde option en matière de mobilisation politique - impliquerait de se fondre par exemple dans la définition officielle du problème public et à se poser en relais des politiques poursuivies par l'OFSP (stricte information du public) ou à (continuer à) fonctionner comme partenaire de terrain de Swisstransplant (promotion du don d'organes). Une telle coopération au sein de l'espace associatif suppose des négociations et une confiance entre des collectifs de taille inégale et au fonctionnement différent, sans compter l'écueil non négligeable

667 Neveu 2015a, 113. 
de la barrière des langues. Une autre voie consiste à se mobiliser pour se faire reconnaître comme un interlocuteur légitime des pouvoirs publics en y exerçant une fonction consultative ou décisionnelle. À l'heure de la démocratie sanitaire, les patients sont de plus en plus légitimes à participer à la gouvernance du système de soins. Signalons à cet égard qu'une association, l'AST, fait partie du comité de suivi du plan d'action national «Plus d'organes pour des transplantations ${ }^{668}$ ». Une autre voie d'intégration consiste dans la professionnalisation des structures et activités associatives. La professionnalisation peut être le résultat d'une reconnaissance par l'État du bien-fondé et de la qualité des prestations de services et d'entraide, impliquant un financement de la part des pouvoirs publics. Si cette option a l'avantage de surmonter les limites du bénévolat, le risque est de rendre l'association dépendante de l'État au point qu'elle n'assume qu'un rôle de gestionnaire au détriment de sa logique militante ${ }^{669}$, ou qu'elle soit réduite à un «rouage d'une politique publique ${ }^{670}$.»

Quelle que soit la stratégie envisagée (contestation ou intégration), la problématique des ressources et de la fragmentation des forces associatives demeure. Confrontées à des difficultés budgétaires ou à une baisse de l'engagement, des associations œuvrant autour d'un même enjeu peuvent être amenées à créer des alliances durables, voire à créer une structure nationale ou régionale unique. On l'a dit, ce type de processus n'est guère aisé. Outre l'implication locale des actions et la personnalisation de l'engagement pour une cause, d'autres obstacles peuvent se dresser: concilier des conceptions différentes de l'action associative ou intégrer de nouveaux acteurs comme des représentants du corps médical ${ }^{671}$.

668 Le choix de cette association plutôt qu'une autre s'est fait sur des critères de visibilité et de représentativité nationale des transplantés. Le rôle du comité de suivi est de discuter, en concertation avec le comité de pilotage, les propositions de l'OFSP relatives à la réalisation des différents sous-projets (entretien avec Mme Nyfeler, coresponsable de la section transplantation et procréation médicalement assistée, OFSP).

669 Girard 2014, Pinell 2002.

670 Neveu 2015a, 67.

671 Bach 1995. 
Dans le cas présent, malgré des échecs dans le passé, l'idée de créer une association faîtière reste d'actualité, et est défendue tant par Swisstransplant que par certains acteurs associatifs, conscients de la nécessité de parler d'une seule voix et d'unir les forces en présence. Au-delà de la capacité d'action des collectifs de patients, l'enjeu de la mobilisation est de donner une visibilité consensuelle au don d'organes, thème particulièrement sensible aux voix discordantes dans l'espace public. 


\section{Partie III. \\ Enjeux actuels et perspectives}

Vincent Barras, Manuel Pascual et Raphaël Hammer

En l'espace d'un peu plus d'un demi-siècle, la technique médicale de la greffe a connu un développement majeur, aussi bien sur les plans scientifique et clinique que politique et sociétal. Les chapitres précédents se sont attachés à comprendre de quelle manière la médecine de transplantation et le don d'organes étaient devenus l'enjeu public que l'on connaît aujourd'hui dans le contexte helvétique. L'analyse socio-historique a notamment mis en évidence les processus informels et le rôle clé des initiatives individuelles de certains médecins ou chirurgiens dans l'émergence des programmes de transplantation dans les hôpitaux suisses à la fin des années 1960. Tout en étant encadrés par les textes de l'ASSM, ces premiers pas ont été accomplis en dehors de toute impulsion hiérarchique et de tout plan directeur politique ou hospitalier concerté. Les prémices organisationnelles de la transplantation en Suisse dans la période 1950-1980 ont été le fait d'un développement médical largement autonome; cette autonomie s'amenuisera par la suite avec les transformations des modes de gouvernance des hôpitaux.

Schématiquement, les décennies suivantes sont caractérisées par l'extension et la consolidation de l'organisation du don et de la transplantation, notamment au niveau de la gestion des échanges 
d'organes entre hôpitaux. Un des points d'orgue de cet essor est la création en 1985 de la fondation Swisstransplant qui permettra le passage d'une coopération informelle et décentralisée à l'établissement d'une coordination nationale visant à optimiser l'utilisation des organes disponibles. Cette évolution est étroitement liée aux exigences d'un espace politique national du don et de la transplantation d'organes que pose la perspective d'une régulation fédérale et non plus cantonale. Le début des années 2000 marque un tournant majeur avec notamment l'entrée en vigueur en 2007 de la LTx, consécutive à l'acceptation en 1999 de l'article constitutionnel 119a donnant la compétence aux autorités fédérales de légiférer sur ce domaine d'activité. Cette nouvelle donne politique modifie substantiellement le champ du don d'organes en redéfinissant les rôles du Conseil fédéral, de l'OFSP et de Swisstransplant. Au terme de l'analyse sociologique, ce changement d'échelle de la politisation du don d'organes comme problème public apparaît comme l'un des facteurs qui expliquent «l'essoufflement» de la mobilisation de nombre d'associations de patients transplantés, en Suisse romande comme dans les autres régions linguistiques.

Le domaine du don et de la transplantation a connu au cours des dernières années d'autres développements importants dans la continuité de cette nouvelle dynamique politique, organisationnelle et sociale que la LTx a instituée, et qui préfigurent les enjeux et débats sociétaux et politiques à moyen terme en Suisse. Cette troisième partie de l'ouvrage vise à compléter le regard sociologique et historique en présentant les évolutions les plus récentes du contexte du don et de la transplantation d'organes. Les textes de trois acteurs clés que sont l'OFSP (Dr Andrea Arz de Falco, vice-directrice), Swisstransplant (Dr Franz Immer, directeur ${ }^{672}$ ) et les médecins «sur le terrain » (Pr Philippe Eckert, ancien médecin-chef de service du Service de médecine intensive et adulte, CHUV et Pr Manuel Pascual, médecin-chef de service du centre de transplantation d'organes, CHUV) permettent de prendre connaissance du devenir des différents processus engagés depuis une vingtaine d'années. Leurs contributions

672 La traduction du texte de Franz Immer a été assurée par Alexia Cochand et Vincent Barras depuis l'allemand. 
montrent la diversité des mesures déployées à de multiples niveaux (coordination, optimisation des processus, formation du personnel soignant, plan d'action national, registre national du don d'organes, réseaux hospitaliers, étude de cohorte, évaluation du potentiel de dons...). Elles livrent également une image précise de la complexité du domaine du don et de la transplantation d'organes en Suisse, sur le plan des acteurs et des instances concernés, ainsi que des échelons géographiques, institutionnels, politiques et administratifs qui sont impliqués. En filigrane de cette synthèse des changements actuels du paysage institutionnel de la médecine de transplantation en Suisse, nous pouvons également faire le constat que nombre de questions de fond présentes dès les débuts de l'institutionnalisation du don et de la transplantation d'organes, comme nous avons pu le montrer dans les chapitres précédents, demeurent. Quand bien même elles sont façonnées spécifiquement par le contexte social et historique, des questions comme l'optimisation d'une ressource rare, la pénurie d'organes, les modalités du consentement, le sens du don, les finalités de l'information de la population, ou encore la participation du public et des patients dans le débat social sont toujours d'actualité, et appellent à prolonger la réflexion pluridisciplinaire sur le don et la transplantation d'organes comme enjeu de société. 



\title{
Chapitre 10
}

\section{Développements du point de vue de l'Office fédéral de la santé publique (OFSP)}

\author{
Andrea Arz de Falco
}

\subsection{Aperçu}

Le besoin d'organes à des fins de transplantation est supérieur à l'offre. Cette situation ne se limite pas à quelques pays moins performants dans la détection des donneurs, comme la Suisse; elle est mondiale ${ }^{673}$ et n'épargne pas des pays ayant figure d'exemple tels que l'Espagne et la Croatie. Malgré les efforts déployés, seulement $10 \%$ environ des besoins effectifs sont couverts dans le monde ${ }^{674}$.

Le besoin d'organes solides ne cesse de croître ${ }^{675}$. Grâce aux progrès de la médecine, la transplantation constitue en effet de plus en plus souvent une option thérapeutique; certaines maladies chroniques telles que le "diabète sucré " ou les maladies du foie en phase terminale peuvent désormais être soignées grâce à une transplantation. Mais si les besoins augmentent, la disponibilité des organes à des fins de transplantation doit croître également pour

673 Scheuher 2016.

674 Matesanz et al. 2017.

675 Matesanz et Miranda 2002. 
éviter que l'écart entre l'offre et la demande ne continue de se creuser et que la liste des patients en attente d'une greffe ne s'allonge. Seuls un engagement fort et une volonté politique de prendre des mesures visant à augmenter le nombre d'organes disponibles pour une transplantation sont susceptibles d'offrir aux patients des perspectives appropriées.

En 1999, le peuple et les cantons ont clairement accepté une disposition constitutionnelle sur la médecine de transplantation (art. 119a Cst). Il s'en est suivi l'entrée en vigueur en 2007 de la loi fédérale du 8 octobre 2004 sur la transplantation d'organes, de tissus et de cellules $(\mathrm{LTx})^{676}$ et de cinq ordonnances d'exécution ${ }^{677}$. Ainsi, pour la première fois dans ce domaine, une même réglementation était applicable dans toute la Suisse, introduisant, par exemple, le modèle de consentement au sens large. Au cours des années qui ont suivi, le nombre des donneurs décédés a stagné. Si la barre des 100 donneurs a été franchie en 2009, le taux de 12 donneurs par million d'habitants (pmp, per million population) ainsi obtenu restait bas, se situant dans le dernier tiers à l'échelle internationale. L'effet sur les listes d'attente n'a pas tardé: le nombre de patients en attente d'organes a ainsi brutalement augmenté, passant de 868 fin 2007 à 1478 fin $2017^{678}$.

Y avait-il alors en Suisse un potentiel inexploité? Le cas échéant, pourquoi les donneurs potentiels n’ont-ils pas été détectés?

\section{2 Étude relative au potentiel de la Suisse (SwissPOD)}

Les résultats de l'évaluation formative relative à l'exécution de la LTx, évaluation fondée sur des données relevées entre 2007 et 2009, indiquent que le vivier de donneurs éventuels n'était pas exploité de façon optimale en Suisse et qu'il existait, par conséquent, un net potentiel d'amélioration. On constate notamment une grande

676 www.admin.ch/opc/fr/classified-compilation/20010918/index.html

677 www.admin.ch/opc/fr/classified-compilation/81.html\#810

678 www.bag.admin.ch/bag/fr/home/zahlen-und-statistiken/zahlen-fakten-zu-transplantationsmedizin/zahlen-fakten-zur-spende-und-transplantation-von-organen. html\#1143296152 
disparité, au niveau du nombre de donneurs annoncés, entre les divers hôpitaux effectuant des prélèvements d'organes ainsi qu'entre les différentes régions linguistiques.

L'étude Swiss Monitoring of Potential Organ Donors (SwissPOD) a été lancée en 2009 à l'initiative de l'OFSP ${ }^{679}$ afin d'étudier les raisons de tels écarts.

Le Comité national du don d'organes (CNDO) a assuré la direction de cette étude et le "Groupe des 15 » (aujourd'hui association Médecine Universitaire Suisse) et l'OFSP ont contribué à son financement. Dans le cadre de l'étude de cohorte prospective, les données relatives à l'ensemble des décès d'adultes survenus dans les unités de soins intensifs accréditées et dans environ la moitié des services d'urgences ont été examinées et le processus du don d'organes dans les différents hôpitaux a été évalué entre septembre 2011 et août 2012.

Si l'étude a fait état de bonnes conditions organisationnelles et structurelles au niveau des processus du don d'organes, elle a également mis en évidence de nombreuses lacunes et défaillances: $66 \%$ des donneurs potentiels ont été "perdus» parce qu'ils n'ont pas été identifiés comme tels ou parce qu'ils n'ont pas pu être pris en charge par manque de personnel et/ou de lits. Le taux de refus s'élevait alors à $52 \%$, un niveau très élevé comparé à celui des autres pays européens où le taux moyen avoisine les $30 \%$. Il apparaît, en outre, que l'entretien avec les proches et le moment de la demande de don jouent un rôle décisif dans la prise de décision.

Les auteurs de l'étude ont conclu que, théoriquement, la Suisse avait alors un potentiel de 290 donneurs, soit un taux de 36,5 pmp, c'est-à-dire près de trois fois supérieur au taux effectif à cette période.

\subsection{Un rapport répondant à trois postulats}

En 2010, la question de la pénurie d'organes a également été débattue au Parlement: trois postulats ont été déposés (Gutzwiller

679 Unterschiedliche Organspenderaten in der Schweiz: Eine prospektive Kohortenstudie zu potenziellen Spendern: www.bag.admin.ch/bag/fr/home/strategie-und-politik/politische-auftraege-undaktionsplaene/aktionsplan-transplantationsmedizin.html 
[10.3703], Amherd [10.3701] et Favre [10.3711]) essentiellement pour demander l'introduction de mesures visant à accroître le taux de donneurs. En réponse à ces interventions parlementaires, l'OFSP a transmis en mars 2013 au Parlement un rapport intitulé «Examen de mesures susceptibles d'augmenter le nombre d'organes disponibles pour une transplantation en Suisse $e^{680} »$. Ce rapport analyse l'effet des mesures proposées sur le nombre de donneurs. Il montre notamment que ni l'introduction du consentement présumé, ni la création d'un registre des donneurs, ni la mention de la qualité de donneur sur un document officiel ne sont susceptibles d'accroître sensiblement le nombre de donneurs. La seule mesure prometteuse consiste à investir dans l'information et dans la formation du personnel médical.

Le rapport confirme, en outre, que des mesures isolées ne permettront pas à elles seules d'augmenter durablement le taux des donneurs. Les pays ayant les meilleurs résultats en la matière ont accru le nombre de leurs donneurs surtout grâce à la mise en place d'un plan d'action combinant et coordonnant différentes mesures adaptées.

\subsection{Le plan d'action}

$\mathrm{Au}$ vu des conclusions du rapport répondant aux postulats, le Conseil fédéral a lancé, le 8 mars 2013, le plan d'action «Plus d'organes pour des transplantations ${ }^{681}{ }$. L'objectif était d'améliorer durablement la situation de pénurie en augmentant le nombre de donneurs et par là même le nombre d'organes disponibles. Il s'agissait notamment d'optimiser les processus dans le domaine du don d'organes ainsi que les structures hospitalières afin de mieux identifier et prendre en charge les donneurs potentiels.

Le plan d'action suisse prévoit également la mise en œuvre coordonnée et harmonisée de mesures d'optimisation à l'échelle natio-

680 Bericht in Erfüllung der Postulate Postulate Gutzwiller [10.3703], Amherd [10.3701] und Favre [10.3711]: www.bag.admin.ch/bag/fr/home/strategie-und-politik/politische-auftraege-und-aktionsplaene/aktionsplan-transplantationsmedizin.html

681 www.g2020-info.admin.ch/fr/create-pdf/?project_id=54 
nale. Il fait partie intégrante de la stratégie politique Santé 2020 du Conseil fédéral et plus précisément de l'objectif visant à promouvoir la qualité des prestations et des soins (domaine d'action "Garantir et renforcer la qualité des soins $\left.{ }^{682} »\right)$.

Le plan d'action a pour but concret de porter le taux de donneurs décédés à 20 pmp, un niveau supérieur à la moyenne européenne. Cet objectif, fixé en collaboration avec des experts à partir des résultats de l'étude SwissPOD, est considéré comme ambitieux mais réaliste.

En 2015, le comité de pilotage, organe décisionnel du plan d'action, a décidé de durcir cet objectif: le taux de 20 pmp ne devra être atteint qu'avec les dons de patients décédés de mort cérébrale primaire («Donation after Brain Determination of Death» ou DBD), c'est-à-dire ne pas inclure les dons intervenant après la mort cérébrale secondaire à l'arrêt cardio-circulatoire («Donation after Circulatory Determination of Death» ou DCD) ${ }^{683}$.

\subsection{Organisation du projet}

L'organisation du projet est conduite par l'OFSP, y sont associés les représentants des principaux acteurs dans le domaine du don d'organes comme les cantons (CDS), Swisstransplant/CNDO, les soins intensifs et les centres de transplantation. Outre l'implication des organisations directement concernées, l'OFSP tenait à intégrer au projet des représentants d'une association de transplantés, d'une organisation de patients et de la Commission nationale d'éthique dans le domaine de la médecine humaine (CNE). C'est pourquoi un comité de suivi a été créé, qui devait servir d'organe de réflexion aux décisions du comité de pilotage de l'échelon supérieur. Une réunion commune des deux comités a eu lieu au moins une fois par an, afin de favoriser l'échange, de discuter de la stratégie définie et d'en garantir l'acceptation. Par exemple, les mesures des champs

682 www.bag.admin.ch/bag/fr/home/strategie-und-politik/gesundheit-2020/eineumfassende-strategie-fuer-das-gesundheitswesen.html

683 www.bag.admin.ch/bag/fr/home/medizin-und-forschung/transplantationsmedizin/ spenden-von-organen-geweben-nach-dem-tod/nachweis-des-todes.html 
d'action ont été identifiées en 2013 lors d'un atelier commun des deux comités.

En avril 2013, le plan d'action a été placé sous le patronage du Dialogue Politique nationale de la santé (PNS), plate-forme permanente de la Confédération et des cantons pour les questions de politique de santé, garantie d'un ancrage politique optimal. Le Dialogue PNS a chargé le comité de pilotage, dans un premier temps, de dresser un état des lieux et de définir les priorités et, dans un deuxième temps, de mettre en œuvre les mesures définies dans le cadre de sous-projets.

Les améliorations définies ont été classées en quatre champs d'action:

- formation du personnel médical;

- processus et gestion de la qualité;

- structures et ressources des hôpitaux;

- campagnes et relations publiques.

Pour satisfaire à l'exigence d'une mise en œuvre coordonnée et groupée, les mesures ont été listées dans un modèle d'efficacité (voir Figure 1$)^{684}$.

\subsection{Réalisation}

Depuis 2014, les mesures définies sont mises en œuvre selon une approche coordonnée. Trois des sous-projets (formation, processus et gestion de la qualité et structures et ressources) sont réalisés par Swisstransplant/CNDO, le quatrième (campagnes et relations publiques) étant du ressort de l'OFSP.

Ces efforts ont certes conduit à une augmentation du taux de dons avec un pic en 2015, mais cette poussée a fléchi dès 2016, le nombre des donneurs chutant de manière inexpliquée, notamment

684 www.bag.admin.ch/bag/fr/home/strategie-und-politik/politische-auftraege-undaktionsplaene/aktionsplan-transplantationsmedizin.html 
Figure 1.

Modèle d'efficacité 2013
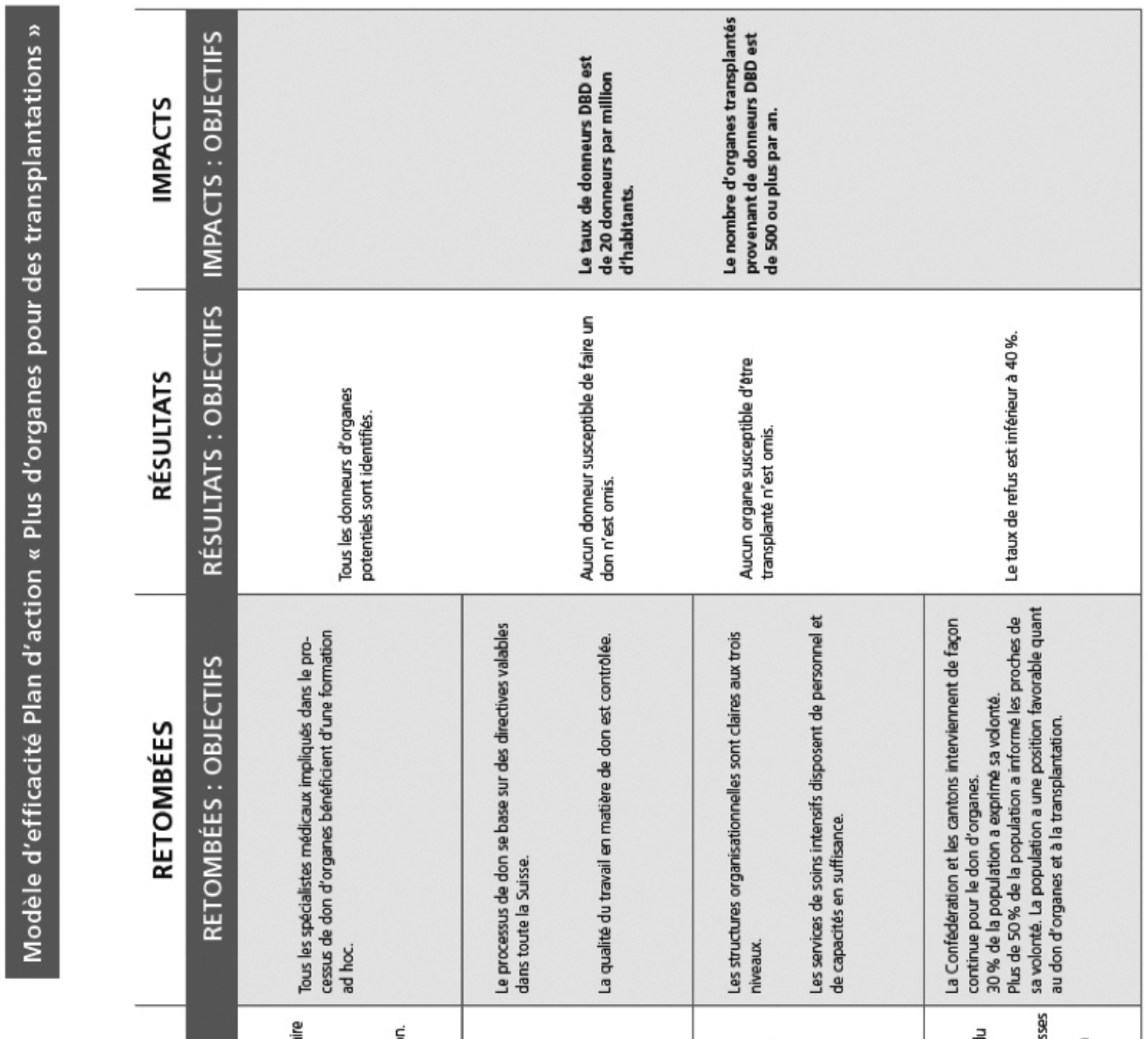
au cours du premier semestre. Ce recul a fait l'objet de diverses hypothèses et suppositions: s'agissait-il simplement de fluctuations naturelles du nombre de dons ou bien le maximum avait-il été atteint? Le soulagement a été grand lorsqu'on enregistra en 2017 une nouvelle tendance à la hausse allant jusqu'à dépasser le niveau de 2015 en fin d'année.

Un premier bilan intermédiaire a été établi fin 2017. Les nombreuses améliorations mises en place, la collaboration plus étroite entre les différents réseaux de don d'organes, une transparence accrue et une garantie de financement du personnel chargé de la coordination sur place ont certes entraîné une augmentation du nombre de dons, mais celle-ci a été plus faible que prévu. Car un problème difficile à expliquer demeure: le taux de refus lors des entretiens avec les proches. Depuis le lancement du plan d'action, il oscille en effet entre $50 \%$ et $60 \%$ dans l'ensemble de la Suisse, un niveau deux fois supérieur à la moyenne européenne. Le taux de refus a fortement augmenté au fil du temps sans jamais fléchir. On constate par ailleurs un énorme écart entre les différents réseaux.

Le taux de dons a certes à nouveau sensiblement augmenté début 2018, mais il semble que l'on ait été trop optimiste dans les objectifs que l'on s'est fixés et que le taux de 20 pmp pourra difficilement être atteint d'ici à fin 2018 en prenant en compte uniquement les dons intervenant après la mort cérébrale primaire ${ }^{685}$ (DBD).

Les objectifs n'ont pas été atteints, d'une part, parce que certaines mesures n'ont pas encore été entièrement réalisées, d'autre part, parce qu'il s'est avéré que certaines actions demandaient plus de temps pour déployer leurs effets.

\subsection{Prolongation du plan d'action}

Le bilan du plan d'action est globalement très positif: il a permis de nombreuses avancées en termes de mise en réseau, d'optimisation des processus, de mise en place de structures et d'acquisition de

$685 \mathrm{Au} 31$ décembre 2019, le nombre de donneurs décédés s'est établi à 18,4 pmp (11,7 pmp si l'on ne tient compte que des donneurs en état de mort cérébrale, dits DBD) (Swisstransplant, Preliminary Statistics 2019). 
compétences. Une base solide est ainsi en place pour les années à venir. Pour que les mesures prévues portent leurs fruits de manière optimale, le Dialogue PNS a décidé en mai 2018 de prolonger la durée du plan d'action jusqu'en 2021. La prolongation ne doit toutefois pas uniquement servir à cette seule fin, elle doit également permettre d'approfondir ou d'adapter les différentes mesures et de traiter le sujet des donneurs décédés suite à un arrêt cardiaque (DCD).

Parallèlement à cette décision, le Dialogue PNS s'est félicité de la proposition visant à clarifier les raisons motivant la décision lors de l'entretien. Une enquête nationale rétrospective doit être réalisée auprès des familles dans un délai de six à douze mois après le décès d'un donneur potentiel. On attend de ce sondage téléphonique qu'il apporte des informations approfondies sur le processus décisionnel et qu'il renseigne sur les motifs de la décision. Cela permettra de définir des mesures en vue d'optimiser les entretiens avec les proches puis de les mettre en œuvre.

\subsection{Perspectives}

Le besoin de coordination demeurera après l'échéance de la prolongation du plan d'action; c'est pourquoi il est prévu de transférer celui-ci vers un programme permanent à partir de 2022, comme cela a été fait dans d'autres pays qui obtiennent de bons résultats en matière de dons d'organes. L'offre sera toujours inférieure aux besoins; à cet égard, l'important est de maintenir un certain équilibre entre l'offre et la demande. C'est seulement en poursuivant la promotion du don d'organes que le sujet restera d'actualité et que le patient inscrit sur la liste d'attente pourra espérer obtenir ce qui est essentiel pour lui. 



\section{Chapitre 11}

\section{Développements du point de vue de la fondation Swisstransplant}

\section{Franz Immer}

C'est avec l'entrée en vigueur de la LTx et les ordonnances du $1^{\text {er }}$ juillet 2007 que Swisstransplant a été mandaté en qualité de service national des attributions par l'OFSP. En plus de la gestion de la liste d'attente, il s'agissait, avant tout, d'assurer l'attribution des organes à l'échelle nationale en conformité avec la loi. Selon le principe de base de l'égalité des chances, tous les individus inscrits sur la liste d'attente doivent bénéficier des mêmes chances de recevoir un organe. Le système d'attribution national a remplacé les règlements cantonaux en vigueur jusque-là et l'attribution régionale des organes qui y était liée. Les patients et patientes en situation d'urgence sont prioritaires dans toute la Suisse: le législateur a en effet décidé que les organes disponibles devaient être attribués en priorité aux receveurs dans une situation d'urgence. Alors que l'efficacité médicale constitue le deuxième critère, les groupes d'experts de Swisstransplant ont été chargés d'élaborer un algorithme spécifique pour chaque organe, et destiné à réguler en dernier lieu l'ordre d'attribution en tenant compte du temps d'attente des receveurs potentiels. Un travail herculéen pour ces groupes d'experts constitués par organe. Comment un bien aussi rare qu'un organe peut-il être attribué de manière juste, et pourquoi un système informatisé, 
le Swiss Organ Allocation System (SOAS), devrait-il remplacer le libre choix d'attribution fondé sur l'expertise des spécialistes de la transplantation? Les premiers débats apparus lors de la mise en place de ce système se sont peu à peu apaisés. Les groupes d'experts du Comité médical de Swisstransplant (CM) ont assumé leur responsabilité et ont continuellement adapté les réglementations aux développements médicaux actuels. Ainsi, l'équité dans la procédure d'attribution s'en est trouvée améliorée.

Avec l'entrée en vigueur de la loi, les cantons ont également dû s'engager. Dans les hôpitaux disposant d'unités de soins intensifs accréditées, des professionnels du don d'organes et de tissus ( «les coordinateurs locaux»), rétribués par les cantons, ont été chargés de veiller aux procédures et aux structures d'identification et de signalement de donneurs et donneuses potentiels, tout comme à la prise en charge des proches. Il s'agit là d'une mesure importante prévue par la LTx pour lutter contre le manque d'organes perdurant depuis de nombreuses années. Les cantons ont toutefois interprété et mis en œuvre très différemment cette nouvelle exigence, si bien que la CDS, sous la présidence du conseiller d'État et futur président du Conseil de fondation Pierre-Yves Maillard, a chargé la Fondation, en 2009, de concrétiser ce mandat. Il s'agissait pour cette dernière d'établir des directives se substituant aux obligations légales des cantons sur le plan du don d'organes et de tissus et de développer, à cet effet, des synergies à l'échelle nationale et d'établir des standards. En raison de ce nouveau mandat, qui se révélera capital, comme la suite le montrera, la fondation Swisstransplant a créé le Comité national du don d'organes (CNDO), pendant du Comité médical (CM), dont le travail concerne la transplantation. Constitué de spécialistes du domaine du don d'organes (soins intensifs et urgences), le CNDO a été ainsi chargé de concrétiser le mandat confié par la CDS. Ce comité a élaboré, en premier lieu, le Swiss Donation Pathway, un manuel rassemblant les normes minimales destinées aux différents secteurs impliqués dans la procédure du don d'organes. L'OFSP et l'association Médecine Universitaire Suisse, l'ancien G15, lui ont également confié, à l'initiative de la direction de Swisstransplant, la réalisation d'une étude visant à évaluer le nombre de donneurs 
potentiels en Suisse. Entre le $1^{\text {er }}$ septembre 2011 et le 31 août 2012, tous les décès survenus dans les unités de soins intensifs accréditées de Suisse ont été compilés dans un registre créé à cette occasion par Swisstransplant (étude SwissPOD). Les résultats ont montré que la Suisse dispose du même potentiel de donneurs d'organes que ses pays voisins. Certains facteurs, comme le taux élevé de refus formulés par les proches lors des entretiens, empêchent toutefois le don d'organes d'être finalement concrétisé. Face à cette situation, la Confédération et les cantons ont lancé entre 2013 et 2018 le plan d'action "Plus d'organes pour des transplantations", auquel Swisstransplant et les experts du CNDO ont été activement associés. Dans ce cadre, quatre domaines d'activité ont été identifiés; la gestion de trois d'entre eux incombe à Swisstransplant. Le premier concerne la formation du personnel spécialisé, le deuxième, la mise en place des procédures et des structures dans les hôpitaux. Le troisième se rapporte au financement du personnel spécialisé, dont il a été question plus haut, et à l'exécution du mandat légal qui en découle dans les hôpitaux. Le quatrième a trait à l'information de l'opinion publique, un domaine qui depuis l'entrée en vigueur de la loi dépend de l'OFSP : le Parlement lui a confié la mission d'informer la population de manière neutre sur le thème de la transplantation d'organes. Depuis 2015, la fondation Swisstransplant est partenaire de l'OFSP pour les campagnes d'informations et apporte une expertise par l'entremise du président du CNDO.

La collaboration au niveau suisse s'est sensiblement améliorée. Plus de $80 \%$ du personnel spécialisé dans le domaine du don d'organes et de tissus participe régulièrement à des formations et accomplit un Blended Learning complet, élaboré et financé par la fondation avant même les débuts du plan d'action. En 2018, la Suisse comptait 158 donneuses et donneurs décédés, ce qui correspond à une hausse de $60 \%$ au cours des cinq dernières années et de $50 \%$ en l'espace de dix ans ${ }^{686}$. Cette progression a été considérablement favorisée par la réintroduction du don après arrêt cardio-circulatoire (DCD) à partir du 1er septembre 2011. Jusqu'à l'entrée en vigueur

686 Weiss et al. 2018. 
de la LTx, cette forme de don était surtout pratiquée dans les hôpitaux universitaires de Zurich (USZ) et de Genève (HUG). Les dons après arrêt cardio-circulatoire (DCD) concernent des patientes et patients des unités de soins intensifs dont le pronostic vital est désespéré et dont les soins sont interrompus. On considère qu'un individu décédé d'un arrêt cardiaque dans les cent vingt minutes après l'interruption des soins offre la possibilité d'un prélèvement dit de «Maastricht III» (DCD catégorie III). Les poumons, le foie, les reins et le pancréas peuvent être prélevés et être transplantés avec des résultats aussi bons que dans le cas de prélèvement en situation de mort cérébrale primaire avec maintien de la fonction cardiovasculaire (DBD). Lorsque l'arrêt circulatoire survient dans un laps de temps de cent vingt minutes, on vérifie au moyen d'un examen à ultrason que le cœur n'éjecte plus de sang pendant au moins cinq minutes (réduit de dix à cinq minutes en 2017). Ensuite, la constatation de la mort est effectuée par deux médecins spécialisés, selon le "principe des quatre yeux ", prévu par les directives de l'ASSM. Le patient ou la patiente est alors en état de mort cardiaque et cérébrale.

En raison de la formulation de l'article 8 de la LTx, qui stipule que la volonté du défunt doit être supposée, cette forme de don a été interrompue en juillet 2007 en Suisse: formellement, un donneur «DCD catégorie III » n'est pas décédé au moment du consentement, puisque la mort cérébrale n'est pas encore constatée. Forte de ce constat, Swisstransplant a réuni des représentants des hôpitaux (médecins intensivistes et transplanteurs), de la Confédération, des cantons et de l'ASSM. Tous les participants ont unanimement reconnu que l'interdiction du don «DCD catégorie III » ne se conformait pas à l'objectif poursuivi par le législateur. Par conséquent, une prise de position du Département fédéral de la justice et police a été demandée. Après des travaux préparatoires intensifs, le USZ et les HUG ont réintroduit la forme de don «DCD catégorie III » le 1er septembre $2011^{687}$. Environ sept ans après la reprise de ce type de don, tous les centres de transplantation et la plupart des grands hôpitaux cantonaux, où sont également effectués des prélèvements

687 Wälchli-Bhend et al. 2011. 
multiorganes, y ont recours. Il représente environ $30 \%$ des dons d'organes cadavériques, ce qui contribue considérablement à la hausse du don d'organes et, par là même, de l'activité de transplantation. Entre-temps, les articles 8 et 10, qui concernent les questions relatives aux mesures de conservation des organes, ont fait l'objet d'une adaptation dans le cadre de la révision de la LTx.

Ces avancées ont été possibles grâce à une collaboration intensifiée avec les spécialistes du domaine du don d'organes et de la transplantation. Le don d'organes est partagé en cinq réseaux, coordonnés par cinq médecins cadres sur mandat de Swisstransplant. Le don d'organes est aujourd'hui largement soutenu dans les cinq réseaux de donneurs et bénéficie d'un financement spécial.

Néanmoins, il convient de poursuivre ce développement. La formation des professionnels doit être élargie et approfondie. Durant des années, les étudiants en médecine tout comme ceux du domaine des soins n'ont bénéficié, lorsque cela a été le cas, que d'une formation accessoire au sujet du don d'organes. Ce manque partiel de connaissance a ainsi compliqué la détection et l'annonce de donneurs au sein des hôpitaux. La question du don d'organes et de tissus est un aspect important du "End-of-Life Care ", qu'il est nécessaire de faire connaître avec transparence et compétence grâce à des spécialistes. Le plan d'action de 2013 à 2018 l'a clairement montré: dans plus de la moitié des entretiens menés avec les proches, ces derniers ne connaissent pas la volonté du défunt et doivent, en raison de la législation actuelle, se substituer à sa décision. La situation de tristesse dans laquelle se trouve l'entourage endeuillé, souvent très touché, entraîne un taux de refus nettement plus élevé, de plus de $50 \%$, que dans nos pays voisins.

La fondation Swisstransplant a lancé le $1^{\text {er }}$ octobre 2018 le premier registre national du don d'organes. La décision de donner ou non ses organes et tissus peut être enregistrée tout en restant librement modifiable à chaque instant. Avec cette carte de donneur moderne et électronique, la population bénéficie d'une autre possibilité de consigner sa décision et d'assurer ainsi sécurité et clarté. Connaître les vœux du défunt soulage beaucoup les proches et le personnel de l'hôpital. Les experts sont unanimes; il faut connaître, 
autant que possible, la volonté du défunt pour qu'un climat de confiance soit garanti dans le traitement de cette question complexe et que l'on puisse agir en plein respect.

Pour cette raison, la fondation Swisstransplant soutient également l'initiative populaire lancée par la Jeune chambre internationale Riviera «Pour sauver des vies en favorisant le don d'organes ", qui demande, par modification constitutionnelle, le passage du mode du consentement explicite à celui du consentement présumé. L'initiative a abouti avec 113000 signatures valides et a été déposée en mars 2019 auprès de la Chancellerie fédérale. Une votation populaire aura lieu au plus tôt en 2022, le thème du don d'organes et de tissus reste ainsi présent. Il faudrait donc également continuer à thématiser l'importance de la décision et influencer indirectement le développement du nombre de donneurs.

C'est d'abord l'engagement de nombreux collègues qui a permis la progression des dix dernières années. Le personnel spécialisé des hôpitaux a fait beaucoup, le mandat de la CDS a été crucial, tout comme l'engagement de l'OFSP et des cantons dans le plan d'action. Enfin, les Hôpitaux suisses $(\mathrm{H}+)$ et la Fédération suisse pour tâches communes des assureurs-maladie (SVK) ont créé les conditions-cadres qui ont permis de négocier l'ensemble du processus d'indemnisation des donneurs, qui est garanti à long terme. Les développements positifs que l'on connaît actuellement dans le domaine du don d'organes et de tissus n'auraient, en effet, pas été possibles sans les spécialistes du don d'organes et de tissus actifs dans les hôpitaux, l'engagement des unités d'urgences et celles de soins intensifs tout comme la compensation des frais pour les prestations liées à la procédure du don. Les pressions budgétaires sur les hôpitaux, le manque de personnel spécialisé et les nombreuses exigences administratives auraient pu représenter des obstacles infranchissables. Les normes élevées des hôpitaux en Suisse, l'excellence des équipes de transplantation, tout cela fait que les quelque 1400 patientes et patients actuellement sur la liste d'attente ${ }^{688}$ bénéficient d'une meilleure chance de se voir attribuer un organe.

688 Chiffre correspondant à la situation fin 2019 (Swisstransplant, Preliminary Statistics 2019). 
J'aimerais ici tous les remercier chaleureusement. Durant les dix dernières années, beaucoup de choses ont été faites et le niveau de qualité des hôpitaux suisses a été continuellement amélioré. La considération, la franchise et la transparence de tous les participants impliqués dans ce processus ont rendu possibles ces avancées. Merci. 



\section{Chapitre 12}

\section{Évolution médicale et organisation actuelle}

Manuel Pascual et Philippe Eckert

Sur le plan médical et chirurgical, une évolution des pratiques et des collaborations entre les six centres suisses de transplantation (Bâle, Berne, Genève, Lausanne, Saint-Gall et Zurich) est observée dès le début des années 2000. En effet, dans le passé, un manque de coordination dans le développement des différents types de transplantations d'organes a été constaté en Suisse, comme cela est décrit dans la partie historique de l'ouvrage. Cette problématique complexe d'insuffisance dans la planification ou la coordination des greffes, qui comporte des enjeux organisationnels hospitaliers, médicaux, techniques, économiques ainsi que de "prestige", va donc progressivement être discutée et traitée, initialement au niveau de la CDS, puis par le "Groupe des 15 », sur demande de la CDS. Au début des années 2000, le «Groupe des 15 » (G15) en Suisse est composé des doyens des cinq facultés de médecine et des directeurs généraux et médicaux des cinq hôpitaux universitaires. Aujourd'hui, le G15 a changé de dénomination pour «association Médecine Universitaire Suisse (unimedsuisse) ». 


\subsection{Travaux du Groupe des 15 en Suisse et évolution Vaud-Genève}

Depuis début 2000, le G15 s'est consacré à instaurer progressivement en Suisse une médecine de pointe de haute qualité, dont la médecine de transplantation fait partie intégrante. En janvier 2003, un mandat est ainsi transmis par le G15 au Pr Manuel Pascual (à Boston, puis au CHUV à Lausanne dès mars 2003) en tant que chef de projet d'un groupe de travail composé de personnes de contact pour chaque centre de transplantation suisse et pour Swisstransplant. L'objectif principal de ce mandat est d'établir un concept détaillé de collaboration et de coordination en réseau des activités de la médecine de transplantation en Suisse. Un premier rapport intermédiaire est rendu en avril 2003 suite à des discussions multidisciplinaires et des analyses détaillées de la situation du moment. À la fin de l'année 2004, le rapport final est rendu par le G15 à la CDS, avec des recommandations concrètes visant à planifier et organiser le "Réseau de transplantation suisse multisite ", sur la base des travaux effectués par le groupe de travail du G15. Les aspects principaux du rapport concernent l'optimisation des activités et des collaborations en transplantation, le besoin de concentration de certaines activités (par exemple, procédures d'implantation de l'organe) sur certains sites, l'enseignement, et la coordination de la recherche clinique ainsi que le besoin de créer un registre national.

Dans ce contexte, il est intéressant de mentionner que dès février 2004, les centres de transplantation d'organes de Lausanne et de Genève s'associent pour former officiellement le «Centre universitaire romand de transplantation »(CURT), cela dans le cadre de l'association Vaud-Genève. La répartition des transplantations entre Lausanne et Genève est donc décidée et validée au niveau politique, et se fait désormais selon une logique d'organes (cœur et poumon à Lausanne; foie, pancréas et intestin à Genève). La transplantation rénale, la plus ancienne (qui date du début des années 1970), reste pratiquée sur les deux sites. Au CURT, seuls l'acte chirurgical et le suivi postopératoire immédiat sont concentrés sur un site (au CHUV 
à Lausanne ou aux HUG à Genève). Toutes les autres activités médicales se déroulent sur les deux sites, soit le bilan prétransplantation et le suivi médical posttransplantation à long terme. Cela implique que chaque centre peut continuer à développer une médecine de transplantation de haut niveau (clinique, enseignement, recherche), tout en respectant la répartition des activités de transplantation d'organes.

En 2005, le comité directeur de la CDS accueille favorablement le rapport final du G15 et le modèle proposé pour les transplantations, sous réserve de l'évolution de la planification de la médecine hautement spécialisée (MHS) en Suisse, notamment au niveau intercantonal. À noter que l'élaboration et la finalisation de la "Convention intercantonale relative à la MHS » (CIMHS du 14 mars 2008) prendront un certain temps, avec de nombreuses discussions au niveau politique et médical. Les cantons signent finalement la CIMHS qui entre en vigueur le $1^{\text {er }}$ janvier 2009.

\subsection{Loi fédérale sur la transplantation et développements en Suisse romande et au Tessin}

Comme mentionné aux chapitres précédents, la loi fédérale du 8 octobre 2004 sur la transplantation d'organes, de tissus et de cellules (LTx), ainsi que les ordonnances d'exécution entrent en vigueur le $1^{\text {er }}$ juillet 2007. Cela implique que les mêmes règles sont désormais appliquées pour toute la Suisse, contrairement à la situation antérieure (lois ou règles cantonales). Dans le cadre de la nouvelle loi fédérale, les greffes d'organes doivent être réalisées dans les centres de transplantation qui sont officiellement autorisés par l'OFSP. Le Conseil fédéral, en accord avec les cantons, peut également décider de limiter le nombre de centres de transplantation en Suisse, si cela s'avère nécessaire.

La LTx impose également à tous les hôpitaux disposant d'une unité de soins intensifs des exigences précises en matière d'organisation du don d'organes. Chaque hôpital doit désigner un coordinateur local du don, chargé de mettre en place différentes procédures liées au don: détection des donneurs potentiels, diagnostic de la mort 
cérébrale, maintien du donneur, prise en charge des proches. Ce coordinateur doit également informer et former le personnel hospitalier potentiellement confronté à un patient donneur d'organes. Il assure également un système de contrôle qualité (Registre SwissPOD ultérieurement).

En 2007, au moment de l'entrée en vigueur de la loi, la grande majorité des hôpitaux suisses ne sont pas prêts à assumer les nouvelles obligations qu'ils doivent remplir. C'est notamment le cas en Suisse romande et au Tessin. Forts de ce constat, les chefs des services de soins intensifs des hôpitaux universitaires de Genève (HUG) et du centre hospitalier universitaire vaudois (CHUV), en partenariat avec leurs collègues du CURT et avec le soutien de leur direction hospitalière, lancent les travaux de réflexion, qui vont aboutir à la création d'un réseau hospitalier de don d'organes en Suisse latine: le Programme latin de don d'organes (PLDO).

L'organisation du PLDO (président: Pr Philippe Eckert, Lausanne, puis Dr Deborah Pugin, Genève, en 2020, et Dr Marco Rusca dès 2021) repose sur quelques idées fortes:

- les tâches de coordinateur local du don sont assumées par des infirmier·ère-s spécialistes en soins intensifs ou en soins d'urgence, qui ont un temps dédié pour les effectuer;

- une structure centrale assure le fonctionnement du réseau. Elle est composée d'une direction de cinq membres et d'une coordination de réseau. Celle-ci est constituée de spécialistes du don d'organes qui soutiennent l'activité des coordinateurs de don dans leurs hôpitaux;

- le PLDO est soutenu politiquement par la Conférence latine des affaires sanitaires et sociales (CLASS), qui a décidé de sa création et en a confié la gestion à l'association Vaud-Genève. La CLASS finance le PLDO (jusqu'à l'introduction d'un financement uniforme suisse par Swisstransplant).

Le PLDO a été le premier réseau hospitalier structuré de don d'organes en Suisse. Il est entré en fonction le 1er juillet 2008. Il a rejoint le Comité national du don d'organes (CNDO) créé par Swisstransplant en 2009. 
Durant ses premières années, le PLDO s'est attaché à développer le don d'organes dans tous les hôpitaux, afin notamment de répondre aux exigences de la LTx. Très rapidement une formation spécifique est mise sur pied pour les coordinateurs de don, qui se familiarisent avec tous les aspects de leurs tâches. Grâce à leur engagement, avec le soutien de la coordination générale, des cours centrés sur la communication dans le contexte du don d'organes sont proposés dans les hôpitaux du PLDO. La dynamique positive qui se crée autour du don ainsi que le soutien politique marqué des cantons se traduisent par une augmentation significative du nombre de donneurs d'organes. De plus, en comparaison avec le reste de la Suisse, les cantons latins ont un nombre de donneurs (calculé par rapport à la population) plus élevé, ainsi qu'un taux de refus du don nettement plus bas. Ces résultats s'observent d'abord en étudiant la statistique annuelle de Swisstransplant, ensuite en analysant les résultats de l'étude SwissPOD. Celle-ci, conduite en 2011-2012, a pour objectif d'évaluer tous les décès survenus dans les unités de soins intensifs en Suisse et de rechercher les causes du faible nombre de donneurs (absence de détection, refus des proches, etc.). Cette étude évoluera ensuite vers le registre Qualité SwissPOD qui permet, année après année, de suivre l'évolution du don en Suisse.

Dès la création du CNDO de Swisstransplant, le PLDO a été très actif au niveau national, soutenant le développement d'une organisation suisse du don organisée en réseaux régionaux. S'appuyant sur l'efficacité de son organisation, le PLDO a soutenu la définition de standards nationaux, que ce soit dans l'organisation du don ou la formation des professionnels. Les échanges entre réseaux, qui se sont progressivement développés en une décennie en Suisse, ont permis l'organisation de nouveaux programmes de don, par exemple le don à cœur arrêté (patient en mort cérébrale après arrêt cardiocirculatoire, DCD), ainsi que la mise sur pied de manifestations similaires dans différentes régions, comme la Journée des proches. Celle-ci se veut un moment de partage entre les familles de donneurs et les professionnels du don et rencontre, année après année, un beau succès. 
Après Zurich et Saint Gall, Genève met en place, dès 2016, un programme de don à cœur arrêté (programme DCD). Ce don diffère du don habituel en Suisse, dit à cœur battant (programme DBD). Dans ce dernier cas, le patient est en mort cérébrale, suite à une lésion primaire affectant son cerveau, avec une atteinte irréversible de toutes les fonctions cérébrales. Dans ce cas, la respiration est assurée par un ventilateur relié au patient par un tube oro-trachéal, et la circulation sanguine se poursuit grâce à l'activité cardiaque du donneur, souvent soutenue par des médicaments. Dans le cas du don à cœur arrêté DCD, chez des patients souffrant d'une atteinte très sévère de la fonction d'un organe vital (cerveau sans critères de mort cérébrale, poumon, cœur), il peut être décidé d'un arrêt de la réanimation médicale, avec évolution vers le décès. Avec l'accord du patient ou de ses proches, la mort cérébrale survenant dans les minutes suivant l'arrêt cardiaque, un don d'organes peut être effectué. Dès 2017, un programme similaire est mis en place à Lausanne. En 2019, les programmes DCD procurent un peu plus d'un tiers des dons d'organes et contribuent ainsi de façon importante à l'augmentation du nombre de donneurs en Suisse.

Dès 2016, Swisstransplant assure le financement des coordinateurs de don dans toute la Suisse. Cependant, la CLASS décide de maintenir son soutien financier au don d'organes et au PLDO, en lui confiant un mandat précis: développer les programmes DCD et de don de cornée dans les hôpitaux non universitaires et élaborer un programme d'actions destinées à promouvoir le don en Suisse latine.

Dans un esprit marqué par la solidarité et l'engagement, les coordinateurs locaux de tous les cantons mettent sur pied différentes actions de promotion du don: présence lors de manifestations sportives ou culturelles, exposés dans les écoles, promotion $\mathrm{du}$ don lors des cours obligatoires de sauveteurs, actions dans les entreprises, etc. Des programmes DCD sont aussi déployés dans les hôpitaux de Fribourg puis Sion, alors que le don de cornée est organisé à l'Ospedale Civico de Lugano. Enfin, grâce à ce mandat, le PLDO peut étoffer sa structure de soutien, notamment par un secrétariat, devenu indispensable au vu des nombreux programmes en cours. 
En 2018, le PLDO a fêté ses 10 ans d'activité. Cette organisation maintenant bien rodée entend poursuivre durant les prochaines années l'ensemble de ses programmes en faveur du don d'organes et de tissus.

\section{3 Étude de cohorte suisse de transplantation (STCS)}

En 2004-2005, des discussions préliminaires ont lieu parmi un groupe de cliniciens-chercheurs (P. Meylan, N. Müller, M. Pascual, J. Steiger, C. Van Delden) de divers centres de transplantation suisses, intéressés et ayant des responsabilités directes dans la prise en charge des patients transplantés en Suisse. Il apparaît alors clairement qu'il existe une forte volonté de vouloir mettre sur pied une étude fédératrice de type «cohorte», multicentrique, inspirée en partie du modèle de l'étude cohorte HIV qui a donné de très bons résultats en Suisse depuis ses débuts en 1988. Le projet multicentrique de la Swiss Transplant Cohort Study (STCS) est ainsi lancé (principal investigateur: Pr Jürg Steiger, Bâle; chairman du comité scientifique: Pr Nicolas Müller, Zurich), dont les objectifs principaux sont de récolter prospectivement des données cliniques et paracliniques de qualité, ainsi que des échantillons sanguins, de tous les patients receveurs d'une transplantation en Suisse. Le but principal de cette récolte prospective d'informations détaillées est de pouvoir mener des études scientifiques (real life) de haute qualité, cela sur des nombres de patients relativement élevés. D’autre part, le projet STCS, qui démarre en mai 2008 avec le premier patient enrôlé, respecte les exigences de la législation suisse en vigueur, vu que certaines des données collectées constituent des informations qui sont requises par la LTx, et sert également de registre pour suivre les résultats (survie, complications, etc.) des transplantations en Suisse. Un responsable du projet STCS est identifié dans chaque centre, qui supervise le bon fonctionnement de l'étude, avec le·la coordinateur-rice STCS du centre. Les données sont collectées de manière codée et centralisée (Bâle), et les patients décident de participer ou non au projet STCS, après avoir été informés oralement et par écrit du déroulement et des détails du projet. Le financement est 
assuré depuis les débuts par le Fonds national suisse de la recherche scientifique et par l'association Médecine Universitaire Suisse (cinq hôpitaux universitaires et facultés de médecine suisses, et l'hôpital de Saint-Gall). À noter que cette association Médecine Universitaire Suisse (anciennement G15) est l'organe responsable du projet STCS depuis ses débuts, et que son rôle a été essentiel dans le succès et le suivi du projet qui à ses débuts (2004-2005) s'est développé parallèlement au concept du "Réseau de transplantation suisse multi-site » du G15 (voir 12.1).

\subsection{Médecine hautement spécialisée en Suisse depuis 2009}

C'est donc dans ce nouveau cadre organisationnel en plein changement (LTx, autorisations de l'OFSP nécessaires pour transplanter des organes dans un centre de transplantation, étude de cohorte suisse de transplantation STCS, ainsi que décision de planification de la médecine de transplantation dans le contexte de la CIMHS) que les transplantations d'organes vont évoluer dès 2009-2010 en Suisse. Bien entendu, Swisstransplant, la fondation nationale suisse pour le don et la transplantation d'organes (directeur: Dr Franz Immer, Berne), reste au centre de tout le système en tant qu'organisme national (Service national des attributions, mandaté par l'OFSP). En particulier, c'est Swisstransplant qui attribue de manière centralisée les organes des donneurs décédés aux receveurs, conformément à la LTx, et qui gère les listes d'attente. Swisstransplant et son CNDO organisent les processus du don d'organes sur le territoire suisse.

Le 15 mai 2009, l’Organe de décision MHS élit et nomme le premier président (Pr Peter Suter, Genève) et les premiers membres de l'Organe scientifique MHS (groupe d'experts suisses et internationaux). Le rôle de l'Organe scientifique MHS est d'analyser les questions scientifiques et médicales dans une perspective de MHS, puis de proposer une répartition des activités et des prestations MHS en Suisse, y compris des transplantations d'organes (en tant que domaine identifié MHS). Les propositions doivent être adéquates, basées sur des preuves scientifiques et les solutions doivent être si 
possible consensuelles, afin de faciliter leur acceptation et éviter des recours éventuels. Ainsi les six centres de transplantation suisses (les cinq hôpitaux universitaires et l'hôpital cantonal de Saint-Gall) se voient attribuer officiellement certaines transplantations d'organes, c'est-à-dire l'acte chirurgical de la greffe (implantation de l'organe) et la période péri-greffe pour un organe donné. Comme cela se fait au CURT depuis 2004, les activités médicales (par exemple, les bilans avant transplantation et les suivis à moyen ou long terme des patients greffés) peuvent en principe se poursuivre dans tous les centres de transplantation suisses, sous réserve d'une bonne coordination avec le centre transplanteur désigné (travail de réseau).

Les travaux en relation avec les prestations de la MHS prennent un certain temps, et la décision du «rattachement» des transplantations d'organes adultes à la MHS se fait par l'Organe de décision MHS le 13 septembre 2016, sur la base d'un rapport final élaboré par l'Organe scientifique MHS. La décision finale officielle «d'attribution» des mandats de prestations MHS (procédures d'implantation des organes greffés chez l'adulte) se fait par l'Organe de décision MHS (décision du 19 avril 2018, publiée dans la Feuille fédérale), sur la base du rapport explicatif pour l'attribution des prestations (transplantations d'organes chez l'adulte) élaboré par l'Organe scientifique MHS.

À noter que tous les processus MHS traitant d'un domaine spécifique doivent obligatoirement se faire en deux temps, c'est-à-dire d'abord le « rattachement » du domaine à la MHS, puis, séparément et dans un second temps, «l'attribution des mandats de prestations » du domaine MHS à un ou plusieurs centres donnés.

Ces décisions d'attribution des transplantations d'organes chez l'adulte (rein, cœur, poumon, foie, pancréas et îlots de Langerhans) aux centres de transplantation sont valables jusqu'au 30 juin 2024. Les centres doivent remplir certaines conditions pour pouvoir fournir les prestations spécifiques (remise d'un rapport annuel avec des données recueillies dans le cadre de l'étude-registre STCS, renouvellement des autorisations de l'OFSP tous les cinq ans pour les activités de transplantation, identification d'un médecin responsable du centre de transplantation, etc.). 
En conclusion, le début du XXI ${ }^{\mathrm{e}}$ siècle a donc vu une évolution et des développements très significatifs dans l'organisation du don et de la transplantation d'organes en Suisse. Ces changements, qui étaient nécessaires, ont pour objectif principal l'optimisation des pratiques dans ce domaine MHS qui reste en évolution permanente. 


\section{Références}

\section{Sources}

\section{Archives cantonales vaudoises}

Fond du service de la santé publique:

SB 124/263, «Hôpital cantonal: service de chirurgie A, fonctionnement du service, service de chirurgie, achat de matériel et appareils, voyages d'études et congés prolongés, service de chirurgie pédiatrique, service de chirurgie générale.»

SB 124/261, «Hôpital cantonal: service de chirurgie A, correspondance générale, 1969-1971, [...].»

SB 124/264 «Intervention sur les cadavres, base légale vaudoise, prélèvements d'organes.»

SB 124/202, «Dossiers généraux - 1 correspondance générale.»

Collège des chefs de service du CHUV 1966-1979.

\section{Archives centrales des HUG}

Boîtes: «Direction générale, DG: Marcel Haas, services médicaux, département chirurgie, 0501.»

«Directeur général Marcel Haas, transplantations, 0037.»

Classeur: "Collège des chefs de service, $1 \mathrm{~A}$.»

Procès-verbaux de la sous-commission médicale, 1953-1994.

\section{Archives de la direction générale du CHUV}

Boîte 58: "Collège des chefs de service (CCS) (P-V, corresp. + divers de 1989 à 1995). »

Boîte 76: «Divers, chirurgie, organisation du service de... 1958/1965.» 
Boîte 232: «1982-2001 transplantations 1988-2000, recherche chirurgicale 1994-2001, endoscopie 1982-1998.»

Procès-verbaux de la direction générale 1975-1995.

\section{Archives de l'État du canton de Berne}

Insel II 1 à 43 (1958-1977): «Insel Direcktion (inkl. Akten). » Insel II 861 à 914 (1978-1989): «Direktion, Protokolle und Akten. »

Insel II 82 à 95 (1953-1977) : «Verwaltungsrat und Verwaltungsauschuss Insel Behörden.»

Insel II 105 à 111 (1976-1977): «Veraltungsauschuss (inkl. Akten).»

Insel 934 à 958 (1978-1989) : «Verwaltungsausschuss, Protokolle und Akten. » Insel II 113 à 115 (1976-1977): "Verwaltungsrat (inkl. Akten). »

Insel II 915-932 (1979-1989): «Verwaltungsrat, Protokolle und Akten. » Insel II 979 (1976-1989) : « Ärztekollegium. »

Insel II 982 «Medizinische Fakultät, Chirurgenkollegium / Assistenzärzte (1966-1989).»

Insel II 711 : «Ärztekollegium : Protokolle. »

Insel II 712: «Ärztekollegium: Protokolle 1966-1983, Diverse Akten 19541974.»

Insel II 1022: «Abteilung für Transplantationschirurgie. »

\section{Archives de l'hôpital de Saint-Gall}

«Kantonspital St. Gallen, Protokoll Chefärtztekonferenz », 1965-1989.

\section{Staatsarchiv des Kantons Basel-Stadt}

SD-REG 5a 0.69.0 (2), «Anzug H. Hofer und Konsorten betr. Schaffung von gesetzlichen Grundlagen für die Transplantation von menschlichen Organen; Anzug G. Eichenberger betr. Vorschriften in den Basler Spitälern betr. Organverpflanzung, 1969-1973.»

SD-REG 11a 3-2-1 (1), «Direktionssitzungen 1962-1994. »

SD-REG 11a 3-4-1 (1), «Chefärtzekonferenz, 1958-1994. »

SD-REG 11a 5-1-12 (1), «Spitalkommission, 1983-1993. » 


\section{Staatsarchiv St.Gallen}

«Jahresbericht über das Kantonsspital St.Gallen ", 1960-1993.

A 340, 1.2.16: «Protokolle der Spital Kommission im KSSG », 1980-1989.

\section{Académie suisse des sciences médicales}

Procès-verbaux des séances du Sénat: 1943-2000.

Procès-verbaux du Comité: 1952-1989.

Classeurs: «Versand der Richtlinien (Todesdiagnose) »; "Subkommision RL zur Hirntodkriterien: Aussdengung ZEK, Aussdenkung SUBK, Richtlienen $(\mathrm{d}+\mathrm{f})$, Prototokolauszug, Mitglieder, etc.»; "Subkommission RL zur Hirntodkriterien: 1. Senatsvernehmlassaung, Stellungnahme Senat, 1. Ausschreibung Saez, Reaktion auf 1. Ausschreibung "; «Subkommission RL zur Hirntodkriterien: RL Entwürfe, 2. Ausschreibung Saez, Replik Frutiger »; «RL "Definition zur Festellung des Todes im Hinblick auf Organtransplantation", Cardiologie Expertenteam.»

\section{Swisstransplant}

Boîtes «Stiftungsrat 88-92»; "Conseil de fondation/comité exécutif 19911992 »; "Conseil de fondation, 1989-1993»; «Stifungsgerat 85-97, 92-94 »;

«Conseil de fondation 1994 »; "Conseil de fondation 1997/1998.»

\section{Revues médicales}

Médecine et Hygiène, 1950-1995.

Praxis, 1950-1995.

Bulletin des médecins suisses, 1950-1995.

Revue médicale de la Suisse romande, 1950-1995. 


\section{Bibliographie}

Abadie Alberto et Gay Sebastien, "The impact of presumed consent legislation on cadaveric organ donation: A cross-country study ", Journal of Health Economics, 25, 2006, p. 599-620.

Akrich Madeleine et Rabeharisoa Vololona, «L'expertise profane dans les associations de patients, un outil de démocratie sanitaire", Santé publique, 24(1), 2012, p. 69-74.

Akrich Madeleine, Méadel Cécile et Rabeharisoa Vololona, Se mobiliser pour la santé. Des associations de patients témoignent, Paris, Transvalor Presses des mines, 2009.

Alexandre Guy P.J., «From the early days of human kidney allotransplantation to prospective xenotransplantation », dans: Paul L. Terasaki (éd.), History of Transplantation' Los Angeles, 1991, p. 337-377.

Alvarez Fernando, "Transplantation d'organes: enjeux éthiques et sociaux", dans: Sylvaine De Plaen (dir.), La Transplantation d'organes. Enjeux et paradoxes, Montréal, 2006, p. 13-23.

Amey Patrick et Hammer Raphaël, «Témoignages et don d'organes : émotions et narration dans la presse suisse romande ", dans: Hélène Romeyer (éd.), La Santé dans l'espace public, Rennes, 2010, p. 107-116.

Bach Marie-Anne, «La sclérose en plaques entre philanthropie et entraide: l'unité introuvable ", Sciences sociales et santé, 13(4), 1995, p. 5-36.

Balsiger Philip, "The Land of Opportunities? Social Movement Studies in Switzerland", dans: Olivier Fillieule et Guya Accornero (éds), Social Movements Studies in Europe, New York, 2016, p. 288-302.

Barbot Janine, «L'engagement dans l'arène médiatique. Les associations de lutte contre le sida ", Réseaux, 17(95), 1999, p. 155-196.

Barbot Janine, Les Malades en mouvements, Paris, 2002.

Bardin Laurence, L'Analyse de contenu, Paris, 1996.

Barkan Leonard, "Cosmas and Damian: of Medicine, Miracles, and the Economies of the Body", dans: Stuart J. Youngner, Renée C. Fox et Laurence J. O’Connell (éds), Organ Transplantation: Meanings and Realities (Madisson 1996), p. 221-251.

Barthe Yannick, Le Pouvoir d'indécision. La mise en politique des déchets nucléaires, Paris, 2006.

Bateman Simone, "Le corps comme outil thérapeutique », dans: Isabelle Baszanger, Martine Bungener et Anne Paillet (dir.), Quelle médecine voulons-nous?, Paris, 2002, p. 175-188. 
Baszanger Isabelle, "Les maladies chroniques et leur ordre négocié », Revue française de sociologie, 27(1), 1986, p. 3-27.

Bellanger Silke et Steinbrecher Aline, «La mort n'est pas la mort: directives médicales et échos de presse au sujet de la mort cérébrale en Suisse entre 1960 et 2000 ", dans: PNR 46 Implants et transplants, Cahier à thèmes $n^{\circ} 1$, Mort cérébrale et don d'organes, Basel, 2002, p. 13-14.

Bellanger Silke et Steinbrecher Aline, "Addressing uncertainties. The conceptualization of brain death in Switzerland 1960-2000 ", dans: Thomas Schlich et Ulrich Tröhler (éds), The Risks of Medical Innovation: Risk Perception and Assessment in Historical Context, Londres, 2006, p. 204-224.

Bellanger Silke, Steinbrecher Aline et Obrecht Sibylle, "Von Herzen und Hirnen. Geschichte des Hirntodes in der Schweiz von 1960 bis 2000 Teil 1 », Praxis. Schweizerische Rundschau für Medizin, 91(45), 2002, 1949-1957.

Bellanger Silke, Steinbrecher Aline et Tröhler Ulrich, «Neue Unsicherheiten des Todes-Diskussion um den Hirntod in der Schweiz in den 1960 ${ }^{\text {er }}$ Jahren ", dans: Alberto Bondolfi, Ulrike Kostka et Kurt Seelmann (éds), Hirntod und Organspende, Ethik und Recht, Nationales Forschungsprogramm Series Volume 1 de Themen Heft, Schweizerischer Nationalfonds zur Förderung der Wissenschaftlichen Forschung, Bâle, 2003, p. 11-25.

Ben-David Orit Brawer, Organ Donation and Transplantation: Body Organs As an Exchangeable Socio-Cultural Resource, Westport, 2005.

Biaudet Julien, Au cour de la greffe: la socialisation secondaire des transplantés cardiaques, thèse de doctorat en sociologie, Université de Lorraine, 2013.

Blumer Herbert, "Social problems as collective behavior", Social Problems, 18(3), 1971, p. 298-306.

Boileau Claire, Dans le dédale du don d'organes. Le cheminement de l'ethnologue, Paris, 2002.

Boltanski Luc, La Souffrance à distance. Morale humanitaire, médias et politique, Paris, 1993.

Bondolfi Alberto, «L'allocation équitable d'organes: critères et priorité », dans: PNR 46 Implants et transplants, Cahier à thèmes $n^{\circ} 5$, La mise en œuvre de la loi sur la transplantation, réponses à des questions ouvertes, Bâle, 2002, p. 5-8.

Bosisio Francesca, Santiago Marie et Benaroyo Lazare, «Financial incentives to improve organ donation: What is the opinion of the Vaud French-speaking population? », Swiss Medical Weekly, 141, 2011, w13312.

Bossi Laura, Les Frontières de la mort, Paris, 2012.

Buckner Clarence D., "Remembering Bruno Speck», Acta Haematologica, 103, 2000, p. 5-6. 
Bury Michael, "The sociology of chronic illness ", Sociology of Health \& Illness, 13(4), 1991, p. 451-468.

Buton François, "Sida et politique: saisir les formes de la lutte", Revue française de science politique, 55, 2005, p. 787-810.

Cameron John S., "Greffes rénales avec donneurs vivants non apparentés: évolution des pratiques cliniques et des considérations éthiques ", Actualités néphrologiques, 2002, p. 309-333.

Cardon Dominique, Heurtin Jean-Philippe, Martin Olivier, Pharabod AnneSylvie et Rozier Sabine, "Les formats de la générosité. Trois explorations du Téléthon ", Réseaux, 95, 1999, p. 15-105.

Carvais Robert et Sasportes Marilyne (éds), La Greffe humaine, Paris, 2000.

Carricaburu Danièle, «L'Association Française des Hémophiles face au danger de contamination par le virus du sida: stratégie de normalisation de la maladie et définition collective du risque ", Sciences Sociales et Santé, XI (3-4), 1993, p. 55-81.

Carricaburu Danièle et Ménoret Marie, Sociologie de la santé. Institutions, professions et maladies, Paris, 2004.

Cefaï Daniel, «La construction des problèmes publics. Définitions de situations dans des arènes publiques ", Réseaux, 14(75), 1996, p. 43-66.

Cefaï Daniel, Pourquoi se mobilise-t-on? Les théories de l'action collective, Paris, 2007.

Cefaï Daniel et Trom Danny (éds), Les Formes de l'action collective: mobilisation dans des arènes publiques, Paris, 2001.

Chamak Brigitte, «Autism and social movements: French parents' associations and international autistic individuals' organizations ", Sociology of Health and Illness, 30(1), 2008, p. 76-96.

Charaudeau Patrick, Le Discours médiatique d'information. La construction du miroir social, Paris, 1997.

Chavot Philippe et Masseran Anne, "Histoires de greffes: permanence d'un récit télévisuel », Culture et Musées, 18, 2011, p. 99-128.

Chavot Philippe, Felt Ulrike et Masseran Anne, «Les technosciences face aux savoirs "populaires": mise en sens et mise en scène de la transplantation d'organes ", Recherches en communication, 15, 2001, p. 149-167.

Chenaux Jean-Philippe, Transplantation d'organes: sauver des vies, Lausanne, 2000.

Cobb Roger W. et Elder Charles D., Participation in American Politics: The Dynamics of Agenda-Building, Baltimore, 1983.

Cochand Alexia, «Diffusion d'un savoir et légitimation d'une pratique thérapeutique dans les revues médicales : l'exemple de la transplantation d'organes 
en Suisse entre 1950 et 1990 », Amnis, 14, 2015. En ligne: https://journals. openedition.org/amnis/2530

Cochand Alexia, «Usage des archives hospitalières contemporaines: le cas de l'émergence et de l'intégration de la transplantation d'organes en Suisse (1960-1990)", Revue suisse d'histoire, 67(2), 2017, p. 238-249.

Colinet Séverine, «Entre implication et désimplication associatives. Cas des personnes atteintes de sclérose en plaques (SEP) », Revue ¿̇ Interrogations?, 16, 2013. En ligne: www.revue-interrogations.org/Entre-implication-et

Cormier Nicholas R., Gallo-Cruz Selina R. et Beard Renee L., «Navigating the new, transplanted self: How recipients manage the cognitive risks of organ transplantation ", Sociology of Health \& Illness, 39(8), 2017, p. 1496-1513.

Déchamp-Le Roux Catherine, "Débats autour de la transplantation d'organes ", Sciences sociales et santé, 15, 1997, p. 99-127.

Dodier Nicolas, Leçons politiques de l'épidémie du sida, Paris, 2003.

Doniger Wendy, «Transplanting Myths of Organ Transplants », dans: Stuart J. Youngner, Renée C. Fox et Laurence J. O’Connell (éds), Organ transplantation: Meanings and Realities, Madison, 1996, p. 194-220.

Donzé Pierre-Yves, Bâtir, gérer, soigner: histoire des établissements hospitaliers de Suisse romande, Genève, 2003.

Engeli Isabelle et Varone Frédéric, «Governing morality issues through procedural policies ", Swiss Political Science Review, 17, 2011, p. 239-258.

Engle David, «Psychosocial aspects of the organ transplant experience: What has been established and what we need for the future ", Journal of Clinical Psychology, 57(4), 2001, p. 521-549.

Epstein Steven, La Grande Révolte des malades, Paris, 2001.

Falomir-Pichastor Juan Manuel, Berent Jacques et Pereira Andrea, «Social psychological factors of post-mortem organ donation: A theoretical review of determinants and promotion strategies ", Health Psychology Review, 7(2), 2013, p. 202-247.

Fassin Didier, «Les économies morales revisitées ", Annales. Histoire, Sciences sociales, 6, 2009, p. 1237-1266.

Feeley Thomas Hugh et Vincent III Donald, «How organ donation is represented in newspaper articles in the United States ", Health Communication, 21(2), 2007, p. 125-131.

Felstiner William L.F., Abel Richard L., Sarat Austin, «L'émergence et la transformation des litiges: réaliser, reprocher, réclamer », Politix, 16, 1991, p. 41-54.

Filippini L., «Klinische Nierentransplantation, Transplantationskolloquium Zürich, 25. und 26. November 1966 ", Praxis, 22, 1967, p. 767-768. 
Fillieule Olivier et Péchu Cécile, Lutter ensemble. Les théories de l'action collective, Paris, 1993.

Fixot Anne-Marie, "Don, corps et dette: une approche maussienne", Revue du MAUSS, 35(1), 2010, p. 477-488.

Fortin Marie-Chantal, Duplantie Andrée, Durand Céline, Faucher Claire, Nicolau Dan et Doucet Hubert, "La vie avec et la mort sans: les représentations de la transplantation d'organes dans la presse écrite québécoise ", Bioethica Forum, 4(1), 2011, p. 17-23.

Fox Patrick, «From senility to Alzheimer's disease. The rise of the Alzheimer's disease movement ", Milbank Quarterly, 67, 1989, p. 58-102.

Fox Renée C. et Swazey Judith P., The Courage to Fail. A Social View of Organ Transplants and Dialysis, Chicago, 1974.

Fox Renée C. et Swazey Judith P., Spare Parts. Organ Replacement in American Society, New York, 1992.

Gateau Valérie, Pour une philosophie du don d'organes, Paris, 2009.

Gentili Marc E., «L'aube des greffes humaines: 60e anniversaire des premières transplantations rénales réussies ", Néphrologie et Thématique, 11, 2015, p. 502-505.

Gilbert Claude et Henry Emmanuel, «Lire l'action publique au prisme des processus de définition des problèmes ", dans: Claude Gilbert et Emmanuel Henry (éds), Comment se construisent les problèmes de santé publique, Paris, 2009, p. 9-33.

Girard Alain, «Réticences au prélèvement d'organes: égoïsme ou résistance au biopouvoir ? Une question de catégorisation ", Sciences sociales et santé, 18(1), 2000, p. 35-68.

Girard Gabriel, "Sida : un monde associatif en crise? ", La Vie des idées, 2014. En ligne: http://www.laviedesidees.fr/Sida-un-monde-associatif-en-crise.html

Godbout Jacques T., «Le don au-delà de la dette », Revue du MAUSS, 27(1), 2006a, p. 91-104.

Godbout Jacques T., "Le don, la dette et l'identité dans le don d'organes", dans: Sylvaine De Plaen (éd), La Transplantation d'organes. Enjeux et paradoxes, Montréal, 2006b, p. 59-77.

Godbout Jacques T., "De la dette à l'identité: le don d'organes et de sang", dans: Philippe Chanial (éd.), La Société vue du don. Manuel de sociologie anti-utilitariste appliquée, Paris, 2008, p. 418-427.

Godbout Jacques T. et Caillé Alain, L’Esprit du don, Paris, 1992.

Goy Jean-Jacques, "La transplantation cardiaque pourquoi et pour quels résultats?", Revue médicale de la Suisse romande, 1993, 5, p. 415-420.

Gusfield Joseph, La Culture des problèmes publics, traduit de l'anglais par et postface de Daniel Cefaï, Paris, 2009. 
Hacking Ian, Entre science et réalité. La construction sociale de quoi?, Paris, 2001.

Hamilton David, A History of Organ Transplantation, Ancient Legends to Modern Practice, Pittsburg, 2012.

Hammer Raphaël, Expériences ordinaires de la médecine, Genève/Zurich, 2010.

Hammer Raphaël, «La représentation médiatique du don et de la transplantation d'organes dans la presse suisse romande ", Communication, 30(1), 2012. En ligne: http://communication.revues.org/index2884.html

Hansen Solveig L., Eisner Marthe I., Pfaller Larissa et Schicktanz Silke, " "Are you in or are you out?" Moral appeals to the public in organ donation poster campaigns: A multimodal and ethical analysis ", Health Communication, 33(8), 2018, p. 1020-1034.

Healy Kieran, Last Best Gifts, Chicago, 2006.

Hennette-Vauchet Stéphanie et Nowenstein Graciela, "Dire la mort et faire mourir. Tensions autour de la mort encéphalique et la fin de vie en France ", Sociétés contemporaines, 75(3), 2009, p. 37-57.

Herpin Nicolas et Paterson Florence, «Le don d'organes et la perception de la mort par les Français: les systémistes et les intégralistes ", dans: Robert Carvais et Marilyne Sasportes (éds), La Greffe humaine, Paris, 2000, p. 797-814.

Herzlich Claudine et Pierret Janine, Malades d'hier, malades d'aujourd'hui, Paris, 1991.

Hoeyer Klaus, Schicktanz Silke et Deleuran Iran, «Public attitudes to financial incentive models for organs: A literature review suggests that it is time to shift the focus from "financial incentives" to "reciprocity" ", Transplant International, 26, 2013, p. 350-357.

Hofmann Simon, Umstrittene Körperteile. Eine Geschichte der Organspende in der Schweiz, Bielefeld, 2016.

Hogle Linda F., Recovering the Nation's Body: Cultural Memory, Medicine, and the Politics of Redemption, Nouveau-Brunswick, 1999.

Ikels Charlotte, "The Anthropology of Organ Transplantation ", Annual Review of Anthropology, 42, 2013, p. 89-102.

Imperatori Luca, Gachet Christian, Eckert Philippe et Chioléro René, «Dons d'organes et transplantation: qu'en pensent les soignants? ", Revue médicale suisse, 2372, 2001, p. 2463-2467.

Joralemon Donald, "Organ wars: The battle for body parts ", Medical Anthropology Quarterly, 9(3), 1995, p. 335-356.

Jensen Steven J. (éd), The Ethics of Organ Transplantation, Washington D.C., 2011. 
Kaech François, Barras Vincent, Pascual Manuel et Hammer Raphaël, «Le rôle des associations de patients face au don d'organes en Suisse romande ", Revue médicale suisse, 11, 2015, p. 2250-2253.

Kapitz Christiane, "Figures et enjeux de l'éthique dans l'actualité scientifique de la presse quotidienne ", communication présentée au colloque Sciences, médias et société, 15-17 juin 2004, Lyon, ENS-LSH. En ligne: http:// sciences-medias.ens-1sh.fr/article.php3?id_article $=64$

Kinneart Paul, "L'énigme des allogreffes rénales réalisées chez l'homme au début des années 1950 », Revue médicale de Bruxelles, 27, 2006, p. 464-468.

Knobé Sandrine, "Logiques d'engagement des malades dans les associations de lutte contre le cancer", Socio-logos, 4, 2009. En ligne: http://socio-logos. revues.org/2346

Knobé Sandrine, "Parcours et interactions associatives en contexte de lutte contre le cancer du sein. Des freins à la politisation des discours ", Sociologie Santé, 33, 2011, p. 253-271.

Küss René, "Human Renal Transplantation Memories 1951 to 1981 », dans: Paul L. Terasaki (éd.), History of Transplantation: Thirty-five Recollections, Los Angeles, 1991, p. 37-57.

Küss René et Bourget Pierre, Une histoire illustrée de la greffe d'organes, la grande aventure du siècle, Rueil-Malmaison, 1992.

Lafontaine Céline, Le corps-marché. La marchandisation de la vie humaine à l'ère de la bioéconomie, Paris, 2014.

Langlois Anne, "Relations au "corps" et dons d'organes", Laval théologique et philosophique, 54(1), 1998, p. 63-82.

Latté Stéphane, "Des “mouvements émotionnels” à la mobilisation des émotions », Terrains/Théories, 2, 2015. En ligne: http://teth.revues.org/244

Le Breton David, La Chair à vif. De la leçon d'anatomie aux greffes d'organes, Paris, 2008.

Le Breton David, Anthropologie du corps et modernité, Paris, 2011.

Lepresle Élisabeth, «Le consentement présumé du donneur, un paradoxe du langage », Essaim, 17(2), 2006, p. 179-188.

Lochard Yves et Simonet Maud, "Les experts associatifs, entre savoirs profanes, militants et professionnels ", dans: Didier Demazière et Charles Gadéa (éds), Sociologie des groupes professionnels, Paris, 2009, p. 274-284.

Lock Margaret, Twice Dead. Organ Transplants and the Reinvention of Death, Berkeley, 2002.

Lock Margaret, "La transplantation d'organes à la lumière de l'anthropologie: discours contradictoires et transformation des subjectivités ", dans: 
Sylvaine De Plaen (éd.), La Transplantation d'organes. Enjeux et paradoxes, Montréal, 2006, p. 33-58.

Lock Margaret et Nguyen Vinh-Kim, An Anthropology of Biomedicine, Chichester, 2010.

Logan Robert, "Health campaign research", dans: Massimiano Bucchi et Brian Trench (éds), Handbook of Public Communication of Science and Technology, Londres/New York, 2010, p. 77-92.

Loriol Marc, «Faire exister une maladie controversée: les associations de malades du syndrome de fatigue chronique et Internet", Sciences sociales et santé, 21(4), 2003, p. 5-33.

Löwy Ilana, "Le féminisme a-t-il changé la recherche biomédicale ? ", Travail, genre et sociétés, 14,2005 , p. 89-108.

Mader Mélanie, «Une neutralité qui n'a plus de raison d'être », Bioethica Forum, 3(2), 2010, p. 74-76.

Mader Mélanie, Le Don d'organes entre gratuité et modèles de récompense: quels instruments étatiques face à la pénurie d'organes?, Bâle, 2011.

Mandressi Rafael, Le Regard de l'anatomiste. Dissections et invention $d u$ corps en Occident, Paris, 2003.

Matesanz Rafael et Miranda Blanca, «A decade of continuous improvement in cadaveric organ donation: The Spanish model ", Journal of Nephrology, 15(1), 2002, p. 22-28.

Matesanz Rafael, Domínguez-Gil Beatriz, Coll Elisabeth, Mahíllo Beatriz et Marazuela Rosario, "How Spain reached 40 deceased organ donors per million population ", American Journal of Transplantation, 17(6), 2017, p. $1447-1454$.

Mathieu Lilian, La Participation des associations au débat public sur la santé: enjeux et conditions, Lyon, 1999.

Mayrhofer-Reinhartshuber David, Fitzgerald Annelles, Benetka Gerthard et Fitzgerald Robert D., "Effects of financial incentives on the intention to consent to organ donation: A questionnaire survey ", Transplantation Proceedings, 38, 2006, p. 2756-2760.

Mégevand René, "À propos de 16 cas de transplantation rénale à la clinique chirurgicale universitaire de l'hôpital cantonal de Genève ", Médecine et Hygiène, 1972, p. 982-986.

Mehl Dominique, «Le témoin, figure emblématique de l'espace public/privé ", dans: Daniel Cefaï et Dominique Pasquier (éds), Les Sens du public. Publics politiques, publics médiatiques, Paris, 2003, p. 489-502.

Merminod Gilles, «(Re)constituer un espace public et promouvoir le débat sans prendre position ", Bulletin suisse de linguistique appliquée, 98, 2013, p. 129-153. 
Merminod Gilles, "Entre information et incitation. Stratégies et expertises dans les discours publics du don d'organes ", Cahiers de l'ILSL, 42, 2015, p. 121-151.

Moloney Gail et Walker Iain, «Talking about transplants: Social representations and the dialectical, dilemmatic nature of organ donation and transplantation ", British Journal of Social Psychology, 41, 2002, p. 299-320.

Monnais Laurence, Vaccinations. Le mythe du refus, Montréal, 2019.

Morgan Susan E., "The intersection of conversation, cognitions, and campaigns: The social representation of organ donation ", Communication Theory, 19(1), 2009, p. 29-48.

Morin Edgar, «Pour une crisologie», Communications, 25, 1976, p. 149-163.

Mosimann François, "La transplantation hépatique à Lausanne », Revue médicale de la Suisse romande, 1992, 2, p. 121-125.

Moulin Anne-Marie, «La crise éthique de la transplantation d'organes. À la recherche de la “compatibilité” culturelle », Diogène, 172, 1995, p. 76-96.

Murray Jospeh E., "Organ transplantation: The practical possibilities, discussion", dans: Gordon E.W. Wolstenholme et Maeve O'Connor (éds), Ethics in Medical Progress: With Special Reference to Transplantation, CIBA Foundation Symposium, Boston, 1966, p. 54-77.

Murray Joseph E., Surgery of the Soul. Reflections on a Curious Career, Sagamore Beach, 2001.

Nathoo Ayesha, Hearts Exposed: Transplants and the Media in 1960s Britain, Basingstoke, 2009.

Neukom Marius, Corti Valentina, Boothe Brigitte, Boehler Annette et Goetzmann Lutz, «Fantasized recipient-donor relationship following lung transplantations: A qualitative case analysis based on patient narratives ", The International Journal of Psychoanalysis, 93, 2012, p. 117-137.

Neveu Erik, Sociologie des mouvements sociaux, Paris, 2015a.

Neveu Erik, Sociologie politique des problèmes publics, Paris, $2015 \mathrm{~b}$.

Ngatcha-Ribert Laëtitia, Alzheimer: la construction sociale d'une maladie, Paris, 2012.

Noiriel Gérard, Introduction à la socio-histoire, Paris, 2008.

Oliver Michael, Woywodt Alexander, Ahmed Aimun et Saif Imran, "Organ donation, transplantation and religion", Nephrology Dialysis Transplantation, 26, 2011, p. 437-444.

Padioleau Jean G., L'État au concret, Paris, 1982.

Paillé Pierre et Mucchielli Alex, L'Analyse qualitative en sciences humaines et sociales, Paris, 2003. 
Panese Francesco et Barras Vincent, "L'utopie médicale de la réanimation des corps ", Mouvements: sociétés, politique, culture, 45-46, 2006, p. 36-42.

Panofsky Aaron, "Generating sociability to drive science: Patient advocacy organizations and genetic research ", Social Studies of Science, 32, 2010, p. 1-27.

Perret Claude, «Vie et Réanimation », Praxis, 52, 1966, p. 1510-1514.

Perret Virgile, «La législation suisse sur la transplantation: le rôle des facteurs émotionnels dans sa genèse ", dans: Alexandre Flückiger (éd.) Émouvoir et persuader pour promouvoir le don d'organes? L'efficacité entre éthique et droit, Genève, 2010, p. 97-119.

Peters Thomas G., Fischer Jonathan S., Gisch Robert G. et Howard Richard J., "Views of US voters on compensating living kidney donors ", JAMA Surgery, 151, 2016, p. 710-716.

Pfaller Larissa, Hansen Solveig L., Adloff Frank et Schicktanz Silke, " "Saying no to organ donation": An empirical typology of reluctance and rejection ", Sociology of Health \& Illness, 40(8), 2018, p. 1327-1346.

Pinell Patrice (éd.), Une Épidémie politique. La lutte contre le sida en France (1981-1996), Paris, 2002.

Pinell Patrice, "Éléments pour une réflexion sociologique sur le phénomène associatif dans le domaine de la santé », Sociologie Santé, 26, 2007, p. 19-30.

Plumart Valérie, Le Consentement au prélèvement, entre régulation étatique et initiative associative. La fédération des associations pour le don d'organes et de tissus humains dans le système des transplantations: mobilisation, débat public, représentations (1974-2011), thèse de doctorat, Université de Limoges, 2012.

Prottas Jeffrey et Batten Hellen L., "Neurosurgeons and the supply of human organs », Health Affairs, 8(1), 1989, p. 119-131.

Quéré Régis, "Enjeux éthiques des prélèvements d’organes et de tissus: vers un utilitarisme de la mort?", dans: Emmanuel Hirsch (éd.), Traité de bioéthique: II - Soigner la personne, évolutions, innovations thérapeutiques, Toulouse, 2010, p. 657-670.

Quick Brian L., LaVoie Nicole R. et Stone Anne M., "An examination of organ donation in the news: A content analysis from 2005-2010 of the barriers to becoming an organ donor ", dans: Gurch Randhawa (éd.), Organ donation and transplantation: Public policy and clinical perspectives, Rijeka, 2012, p. 11-22.

Rabeharisoa Vololona et Callon Michel, Le Pouvoir des malades. L'association française contre les myopathies et la recherche, Paris, 1999.

Rabeharisoa Vololona et Callon Michel, «Les associations de malades et la recherche (I): des self help groups aux associations de malades », Médecine/ Sciences, 16(8-9), 2000a, p. 945-949. 
Rabeharisoa Vololona et Callon Michel, «Les associations de malades et la recherche (II): Les formes d'engagement des associations de malades dans la recherche en France ", Médecine/Sciences, 16(11), 2000b, p. 1225-1231.

Rabeharisoa Vololona et Callon Michel, "L'engagement des associations de malades dans la recherche", Revue internationale des sciences sociales, 1(171), 2002, p. 65-73.

Ralph Angelique, Chapman Jérémy, Gillis Jamy, Craig Johnathan, Butow Phyllis N., Howard Kirsten, Irving Michelle, Sutanto Bernadet et Tong Alison, "Family perspectives on deceased organ donation: Thematic synthesis of qualitative studies ", American Journal of Transplantation, 14, 2014, p. 923-935.

Renaudet Isabelle, "Médecine et presse médicale en Espagne: entre les savoirs et les pouvoirs (XIX ${ }^{\mathrm{e}}$ siècle-premier tiers du $\mathrm{XX}^{\mathrm{e}}$ siècle) », El Argonauta español, 8, 2011. En ligne: https://journals.openedition.org/ argonauta/81

Rix Bo Andreassen, "Brain death, ethics and politics in Denmark», dans: Stuart J. Youngner, Robert M. Arnold et Renie Schapiro (éds), The Definition of Death: Contemporary Controversies, Baltimore, 1999, p. 227-238.

Rochefort David A. et Cobb Roger W., The Politics of Problem Definition, Shaping the Policy Agenda, Lawrence, 1994.

Romagnoli Simone, "Transplantation cardiaque et image du corps », Ethique et Santé, 1, 2004, p. 194-199.

Sambuc Cléa, "L'efficacité du marché des organes. Une discussion critique ", Revue économique, 1(63), 2012, p. 51-67.

Sanner Margareta A., «Exchanging spare parts or becoming a new person? People's attitudes toward receiving and donating organs ", Social Science \& Medicine, 52(10), 2001, p. 1491-1499.

Sanner Margareta A., "Two perspectives on organ donation: Experiences of potential donor families and intensive care physicians of the same event ", Journal of Critical Care, 22, 2007, p. 296-304.

Scheper-Hughes Nancy, "The Global traffic in human organs ", Current Anthropology, 41(2), 2000, p. 1-22.

Scheuher Cynthia, "What is being done to increase organ donation? ", Critical Care Nursing Quarterly, 39(3), 2016, p. 304-307.

Schlich Thomas, The Origins of Organ Transplantation: Surgery and Laboratory Science, 1880s-1930s, Rochester, 2010.

Schulz Karl-Heinz et Kroencke Sylvia, «Psychosocial challenges before and after organ transplantation ", Transplant Research and Risk Management, 7, 2015, p. 45-58. 
Sellers Marty T., McGinnis Holle, Alperin Melissa, Sweeney John F. et Dodson Thomas F., "Deterrents to organ donation: A multivariate analysis of 766 survey respondents ", Journal of The American College of Surgeons, 226, 2018, p. 414-22.

Sharp Lesley A., "Organ transplantation as a transformative experience: Anthropological insights into the restructuring of the self ", Medical Anthropology Quarterly, 9(3), 1995, p. 357-389.

Sharp Lesley A., Strange Harvest: Organ Transplants, Denatured Bodies, and the Transformed Self, Berkeley/Los Angeles, 2006.

Shaw David, "Lessons from the German organ donation scandal ", Journal of the Intensive Care Society, 14(3), 2013, p. 200-201.

Shaw Rhonda W., "Expanding the conceptual toolkit of organ gifting ", Sociology of Health \& Illness, 37(6), 2015, p. 952-966.

Shepherd Lee, O'Carroll Ronan E. et Ferguson Eamonn, «An international comparison of deceased and living organ donation/transplant rates in opt-in and opt-out systems: A panel study », BMC Medicine, 12, 2014, p. 131.

Siminoff Laura A. et Chillag Kata, "The fallacy of the "gift of life" ", Hastings Center Report, 29(6), 1999, p. 34-41.

Simmons Roberta G. et Simmons Richard L., "Organ-Transplantation: A Societal Problem », Social Problems, 19(1), 1971, p. 36-57.

Snow David A., "Analyse de cadres et mouvements sociaux", dans: Daniel Cefaï et Danny Trom (éds), Les Formes de l'action collective: mobilisation dans des arènes publiques, Paris, 2001, p. 27-49.

Starzl Thomas E., The Puzzle People: Memoirs of a Transplant Surgeon, Pittsburgh, 2003.

Steiner Philippe, "Le don d'organes: une affaire de famille?", Annales. Histoire, Sciences sociales, 59(2), 2004, p. 255-283.

Steiner Philippe, La Transplantation d'organes: un commerce nouveau entre les êtres humains, Paris, 2010.

Stücklin Nicholas, "Comme on a peu d'organes". Une économie morale de la transplantation, l'exemple de la greffe cardiaque en Suisse, mémoire de licence en sciences sociales, Université de Lausanne, 2008.

Swisstransplant, Preliminary Statistics 2019. En ligne: www.swisstransplant. org/fr/info-materiel/statistiques/chiffres-annuels/

Tesnière Alain, Les Yeux de Christophe. L'affaire d'Amiens, Paris, 1993.

Thiel Marie-Jo (éd.), Donner, recevoir un organe. Droit, dîu, devoir, Strasbourg, 2009.

Thouvenin Dominique, "Don et/ou prélèvement d'organes ", Sciences sociales et santé, 15(1), 1997, p. 75-97. 
Tilney Nicholas L., Transplant: From Myth to Reality, New Haven, 2003.

Titmuss Richard M., The Gift Relationship. From Human Blood to Social Policy, New York, 1970.

Traïni Christophe et Siméant Johanna, «Pourquoi et comment sensibiliser à la cause?", dans: Christophe Traïni (éd.), Émotions... Mobilisation!, Paris, 2009, p. 11-34.

Tröhler Ulrich, «Brain death in Switzerland 1960-2000. Handling a medical innovation ", Practical Neurology, 4, 2004, p. 356-361.

Vachon François, Médecin à l'hôpital Claude-Bernard, Paris, 2010.

Veatch Robert M., Transplantation Ethics, Washington D.C., 2000.

Verspieren Patrick, "Confusions et débats autour de la "mort encéphalique” », Laennec, 4(58), 2010, p. 8-20.

Voegtli Michael et Fillieule Olivier, "Constitution, diversification and normalization of a health problem: Organizing the fight against AIDS in Switzerland (1984-2005) », Contemporary Politics, 18(2), 2012, p. 200-212.

Waissman Renée, Le Don d'organes, Paris, 2001.

Wälchli-Bhend Susanna, Beyeler Franziska, Weiss Julius, Immer Franz, «How an existing donation after circulatory death program was grounded and re-started in Switzerland ", Organs, Tissues \& Cells, 14, 2011, p. 25-26.

Warrington Thomas P. et Bostwick Michael J., «Psychiatric adverse effects of corticosteroids », Mayo Clinic Proceedings, 81, 2006, p. 1361-1367.

Weiss Julius, Coslovosky Michael, Keel Isabelle, Immer Franz F., Jüni Peter et Comité national du don d'organes (CNDO), «Organ donation in Switzerland - An analysis of factors associated with consent rate ", PLoS ONE, 9(9), 2014. En ligne: https://doi.org/10.1371/journal.pone.0106845

Weiss Julius, Elmer Andreas, Mahíllo Beatriz, Domínguez-Gil Beatriz, Avsec Danica, Nanni Costa Alessandro, Haase-Kromwijk Bernadette, Laouabdia Karim et Immer Franz, "Evolution of deceased organ donation activity versus efficiency over a 15 -year period: An international comparison », Transplantation, 102(10), 2018, p. 1768-1778.

Wood Bruce, Patient Power? The Politics of Patients' Associations in Britain and America, Buckingham, 2000.

Woodruff Michael et Francis Addisson, «Transplantation: the clinical problem », dans: Gordon E.W. Wolstenholme et Maeve O'Connor (éds), Ethics in Medical Progress: with Special Reference to Transplantation, CIBA Foundation Symposium, Boston, 1966, p. 6-23.

Youngner Stuart J., "Some must die», Zygon, 38(3), 2003, p. 705-724.

Ziegler Jean, Les Vivants et la mort, Paris, 1975. 


\section{Liste des abréviations}

AFIR: Association fribourgeoise des insuffisants rénaux AGIR: Association genevoise des insuffisants rénaux AJMIR: Association jurassienne des insuffisants rénaux ANeDiT: Association neuchâteloise des dialysés et transplantés ASSM: Académie suisse des sciences médicales AST: Association suisse des transplantés AVDIR: Association vaudoise des insuffisants rénaux AVIRT: Association valaisanne des insuffisants rénaux et transplantés

BMS : Bulletin des médecins suisses

CDS : Conférence suisse des directrices et directeurs cantonaux de la santé

CHUV : Centre hospitalier universitaire vaudois

CNDO : Comité national du don d'organes

CTO : Centre de transplantation d'organes du CHUV

CURT: Centre universitaire romand de transplantation

DBD : Donation after Brain Determination of Death (don en état de mort cérébrale primaire, dit aussi don à cœur battant)

DCD : Donation after Circulatory Determination of Death (don à cœur arrêté, dit aussi don après arrêt cardio-circulatoire)

FMH: Fédération des médecins suisses

HUG: Hôpitaux universitaires de Genève

JdG: Journal de Genève 
LTx: Loi sur la transplantation ou loi fédérale sur la transplantation d'organes, de tissus et de cellules

Mঞ̈H: Médecine et Hygiène

OFSP: Office fédéral de la santé publique

PLDO : Programme latin de don d'organes

RMSR: Revue médicale de la Suisse romande

SSPIR: Société suisse des patients souffrant d'insuffisance rénale

STCS: Swiss Transplant Cohort Study

SwissPOD study: Étude Swiss Monitoring of Potential Organ Donors 


\section{Volumes parus dans la collection}

Invalidité en souffrance

sous la direction de R. Darioli, 1999

Y a-t-il des limites éthiques à la recherche scientifique?

sous la direction de P. Proellochs et D. Schulthess, 2000

Prescrire la contrainte?

sous la direction de J. Gasser et B. Yersin, 2000

L'automédication : pratiques banales, motifs complexes

sous la direction de T. Buclin et C. Amon, 2001

La résilience : résister et se construire

sous la direction de M. Manciaux, 2001

Une nouvelle approche de la différence : comment repenser le " handicap"?

sous la direction de R. de Riedmatten, 2001

Les animaux inférieurs et les plantes ont-ils droit à notre respect?

A. Arz de Falco et D. Müller, 2002

Voir et agir. Responsabilités des professionnels de la santé en matière de violence à l'égard des Femmes

sous la direction de L. Gillioz, R. Gramoni, C. Margairaz et C. Fry, 2003

Quand la médecine a besoin d'interprètes sous la direction de P. Guex et P. Singy, 2003

Santé et droits de l'homme. Les malades de l'indifférence, volume 1 sous la direction de Y. Reinharz Hazan et P. Chastonay, 2004

Ethique et responsabilité en médecine

L. Benaroyo, 2006 
Santé et droits de l'homme. Les nouvelles insécurités, volume 2 sous la direction de Y. Reinharz Hazan et P. Chastonay, 2006

Clinique de l'exil. Chronique d'une pratique engagée sous la direction de B. Goguikian Ratcliff et O. Strasser, 2009

Exercer la pédiatrie en contexte multiculturel. Une approche complémentariste du rapport institutionnalisé à l'Autre Y. R. Leanza, 2011

Les enjeux de l'apprentissage médical à l'hôpital. Une étude de cas dans un service d'anesthésie

M. Bourrier avec la collaboration de S. Coll, 2011

Pour une psychiatrie scientifique et humaniste sous la direction de P. Guex et J. Gasser, 2011

Trajectoires de vie dans la grande vieillesse. Rester chez soi ou s'installer en institution?

S. Cavalli, 2012

Performances et défaillances du sujet âgé. Étude anthropologique des recherches sur le vieillissement cérébral

M. Droz Mendelzweig, 2013

Face au temps qui reste. Usages et symbolique des médicaments en fin de vie

R.-A. Foley, 2016

Police et Psychiatrie. Polyphonie des postures professionnelles face à la crise psychiatrique sous la direction de A. Linder, M. Meyer et K. Skuza, 2016

Repenser le genre. Une clinique avec les personnes trans*

D. Medico, 2016

Vaccinations. Le mythe du refus

L. Monnais, 2019

Folie et politique. Santé mentale en mutation au Brésil

P. Brum, 2020 



